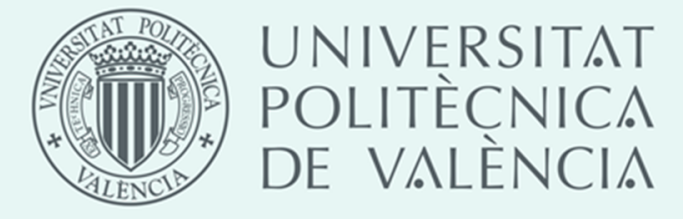

\title{
Structures based on semi-degradable biomaterials for neural regeneration in the central nervous system
}

Manuel Pérez Garnes

PhD Thesis

Supervisors

Manuel Monleón Pradas Jorge Luis Escobar Ivirico

January 2015 


\title{
Structures based on semi-degradable biomaterials for neural regeneration in the central nervous system
}

\author{
Thesis submitted by \\ Manuel Pérez Garnes
}

to obtain the degree of Dr. Eng. Sci.

at the Universitat Politècnica de València

Valencia (Spain), 27 ${ }^{\text {th }}$ January 2015

Supervisors:

Dr. Manuel Monleón Pradas

Dr. Jorge Luis Escobar Ivirico 

A mi familia 
"El conocimiento es patrimonio de la humanidad, no es solo tuyo, transmítelo para beneficio de toda la humanidad" 


\section{AGRADECIMIENTOS}

Agradecer la financiación por parte del Ministerio de Educación y Ciencia por el proyecto CICyT MAT2008-06434 "Materiales para regeneración neural y angiogénesis en el sistema nervioso central" y del Ministerio de Ciencia e Innovación por los proyectos CICyT MAT2011-28791 "Materiales de soporte y liberación controlada para la regeneración de estructuras neurales afectadas por ictus" y PRI-PIMNEU-2011-1372 "Materiales bifuncionales para la regeneración neural de áreas afectadas por ictus".

Agradecer a mis directores de tesis al Dr. Manuel Monleón Pradas y al Dr. Jorge Luis Escobar Ivirico por su dedicación y por compartir sus conocimientos y consejos, así como por su ayuda profesional.

Agradecer a todos los compañeros con los que me he encontrado a lo largo de estos años en el Centro de Biomateriales e Ingeniería Tisular Amparo G., Line, Laura, Carmen A., Chimo, Dunia, Amparo B., Carmen A., Tatiana... y en general a todos por hacer tan agradable el trabajo. Agradecer especialmente a María por hacer más agradable el recorrido durante estos años. Agradecer también a Paloma y Ela por el aire de frescura que me dieron en los últimos momentos de esta tesis y por sus sonrisas. Agradecer especialmente a la Dra. Gloria Gallego Ferrer por confiar en mí y enseñarme el mundo de los biomateriales, al Dr. Manuel Salmerón Sánchez por su siempre buena disposición y apoyo profesional, a la Dra. Ana Vallés Lluch por toda la ayuda durante estos años y por confiar en mí y a la Dra. Laura Teruel Biosca por su y gran labor técnica y organizativa. Agradecer especialmente a José Manuel López Blay por haberme enseñado grandes valores.

Agradecer al personal de microscopía de la Universidad Politécnica de Valencia, Merche, Alicia, Manolo y Jose Luís, y a Salomé del departamento de espectroscopía atómica de la Universidad de Valencia por su asistencia técnica.

Agradecer al Dr. Charles James Kirkpatrick y Dr. Ronald Unger del Institüt für Pathologie, in der Universitätsmedizin der Johannes Gutenberg-Universität Mainz por permitirme trabajar en su laboratorio. Agradecer a los compañeros del instituto, Anne, Thomas, Eva, Tijna..., por su ayuda incondicional. Agradecer especialmente al Dr. Christian Freese por compartir conmigo sus conocimientos sobre cultivos, por su incalculable ayuda y su calurosa acogida.

Agradecer a todos mis amigos Loli, María Dolores, Ana, Inma, Miriam, Elma, Irene, Sergio, Marco..., por todo su apoyo incondicional y por haber estado, más o menos tiempo, pero siempre ahí. Agradecer a mis amigos de travesía por el río Rin Laura y Melina por enseñarme lo que es la valentía y por todos los buenos momentos y a Toni por esos ratos inolvidables y darme una lección sobre los prejuicios. 
Agradecer a toda esa gran familia (es lo que tiene haber tenido cuatro abuelas y otros tantos abuelos) por todo el apoyo. Agradecer a los otros verdaderos autores de esta tesis, a mis padres Miguel y Pilar, porque sin ellos no sería nada, por todo el cariño, por todo el esfuerzo que han hecho por mí, por la gran educación que me han dado, por todo el aliento... Agradecer muy especialmente a mi hermano José Miguel y a mi cuñada Bea por estar siempre ahí y por todo el cariño que me han dado siempre. Agradecer especialmente a David por todos estos años y su comprensión en el período final de esta tesis. 


\section{RESUMEN}

El sistema nervioso central adulto, a diferencia de otros tejidos, posee ciertas limitaciones en cuanto a su regeneración debido a la ausencia de nichos neurogénicos y al microentorno tóxico que se genera tras un daño o enfermedad. Por ello, las pérdidas de células neurales y de reconexión axonal, y por tanto la recuperación de las funciones perdidas, resultan en muchos casos irreversibles. Actualmente existen terapias encaminadas a la regeneración del sistema nervioso central tras sufrir un traumatismo o una enfermedad degenerativa, $y$ éstas consisten en la implantación de células, la liberación de fármacos en el entorno del tejido dañado y la estimulación a través de electrodos. A pesar de que estos métodos han demostrado ciertas mejoras en la regeneración y la recuperación de funciones perdidas, éstas no son suficientes y en la mayoría de los casos producen mejoras transitorias. En este trabajo los esfuerzos se han centrado en el desarrollo de materiales estructurados (andamiajes) que permitan el crecimiento celular en el entorno del sistema nervioso central $y$, especialmente, en el tejido cerebral debido a daños como traumatismos e ictus. Además, estos materiales podrían ser vehículos de células y factores para suplir las poblaciones neuronales y crear un entorno más permisivo para la correcta reconexión axonal.

Para el desarrollo de los andamiajes se pensó en materiales basados principalmente en la sal de sodio del ácido hialurónico, hialuronato. La elección de tal material fue en base a que se trata de un polímero natural presente en muchos tejidos, inclusive el tejido cerebral. Además, el hialuronato y derivados del mismo, que son empleados actualmente en algunas aplicaciones clínicas, han demostrado propiedades biológicas adecuadas para asistir al proceso regenerativo, así como buena compatibilidad y propiedades mecánicas similares a las del tejido cerebral. Sin embargo, el hialuronato sin modificar presenta un bajo tiempo de residencia en el entorno fisiológico debido a su alta solubilidad y rápida degradación, unas propiedades mecánicas difíciles de controlar, un alto grado de hinchado y una difícil manipulación para obtener materiales estructurados. Por todo ello, se han llevado a cabo modificaciones del mismo con el fin de obtener hidrogeles, empleando para ello dos estrategias: el entrecruzamiento con divinil sulfona obteniendo hidrogeles estables y estructurados, y la copolimerización de hialuronato modificado y acrilato de etilo para obtener redes copoliméricas semidegradables.

El entrecruzamiento de hialuronato con divinil sulfona ha sido caracterizado física, mecánica y químicamente con tal de determinar el efecto del entrecruzamiento sobre las propiedades de los materiales desarrollados. Esta química se ha utilizado para el desarrollo de andamiajes porosos estableciendo un método repetitivo para su obtención. Además, se ha estudiado el efecto que tienen los tratamientos posteriores a la reacción de entrecruzamiento en la 
degradación enzimática de los hidrogeles. Por otro lado, se ha estudiado la viabilidad celular de estos materiales en términos de la citotoxicidad y efectos inflamatorios en diferentes células del entorno del sistema nervioso central (astrocitos, neuronas y células endoteliales), así como el efecto que tiene el grado de entrecruzamiento de los andamiajes en la colonización inicial de dichas células.

Las redes copoliméricas basadas en hialuronato y polietilacrilato se han desarrollado con el objetivo de obtener nuevos materiales semi-degradables, combinando las propiedades biológicas del hialuronato y la buena compatibilidad del polietilacrilato con diferentes células, incluyendo las del entorno del sistema nervioso central. Para la reacción de copolimerización fue necesaria una modificación previa del hialuronato, determinando su grado de modificación. Por otro lado, las características antagónicas que poseen ambos materiales permiten controlar las propiedades mecánicas y el grado de hinchado en entornos acuosos variando la composición de las redes copoliméricas. La composición experimental y las propiedades físicas y mecánicas de interés de las diferentes composiciones fueron determinadas por los métodos apropiados en cada caso. Además, se estudió la degradación enzimática de las redes copoliméricas, evaluando los fragmentos liberados así como la integridad del material, con la finalidad de garantizar la estabilidad del mismo durante un hipotético proceso regenerativo. Por otro lado, la viabilidad celular de estos materiales fue evaluada en base a los posibles efectos citotóxicos e inflamatorios sobre células del entorno del sistema nervioso central. Además, la colonización de estas células sobre las redes copoliméricas fue evaluada en estudios preliminares. 


\section{RESUM}

El sistema nerviós central adult, a diferència d'altres teixits, té certes limitacions pel que fa a la seua regeneració per l'absència de nínxols neurogènics i al microentorn tòxic que es genera després d'un dany o malaltia. Per això, les pèrdues de cèl-lules neurals i de reconnexions axonals, i per tant la recuperació de les funcions perdudes, resulten en molts casos irreversibles. Actualment existeixen teràpies encaminades a la regeneració del sistema nerviós central després de patir un traumatisme o una malaltia degenerativa, i aquestes consisteixen en la implantació de cèl-lules, l'alliberament de fàrmacs en l'entorn del teixit danyat i l'estimulació a través d'elèctrodes. Tot i que aquests mètodes han demostrat certes millores en la regeneració i la recuperació de funcions perdudes, aquestes no són suficients i en la majoria dels casos produeixen millores transitòries. En aquest treball els esforços s'han centrat en el desenvolupament de materials estructurats (matrius de suport) que permeten el creixement cel-lular en l'entorn del sistema nerviós central i, especialment, en el teixit cerebral a causa de danys com ara els traumatismes i el ictus. A més, aquests materials podrien ser vehicles de cèl-lules i factors per suplir les poblacions neuronals i crear un entorn més permissiu per a la correcta reconnexió axonal.

Per al desenvolupament de les matrius de suport es va pensar en materials basats principalment en la sal de sodi de l'àcid hialurònic, hialuronat. L'elecció d'aquest material va ser degut a que es tracta d'un polímer natural present en molts teixits, fins $i$ tot el teixit cerebral. A més, I 'hialuronat i derivats del mateix, que són empleats actualment en algunes aplicacions clíniques, han demostrat propietats biològiques adequades per assistir al procés regeneratiu, així com bona compatibilitat i propietats mecàniques similars a les del teixit cerebral. No obstant això, el hialuronat sense modificar presenta un baix temps de residència en l'entorn fisiològic per la seua alta solubilitat i ràpida degradació, unes propietats mecàniques difícils de controlar, un alt grau d'inflament i una difícil manipulació per obtenir materials estructurats. Per tot això, s'han dut a terme modificacions del mateix amb la finalitat d'obtenir hidrogels emprant dues estratègies: l'encreuament amb divinil sulfona obtenint hidrogels estables i estructurats, i la copolimerització de hialuronat modificat i acrilat d'etil per obtenir xarxes copolimèriques semi-biodegradables.

L'encreuament de hialuronat amb divinil sulfona ha estat caracteritzat física, mecànica i químicament per tal de determinar l'efecte de l'encreuament sobre les propietats dels materials desenvolupats. Aquesta química s'ha utilitzat per al desenvolupament de matrius de suport poroses establint un mètode repetitiu per a la seua obtenció. A més, s'ha estudiat l'efecte que tenen els tractaments posteriors a la reacció d'entrecreuament en la degradació enzimàtica dels hidrogels. D'altra banda, s'ha estudiat la viabilitat cel-lular d'aquests materials en termes de la citotoxicitat i efectes inflamatoris en diferents cèl-lules de l'entorn 
del sistema nerviós central (astròcits, neurones i cèl-lules endotelials), així com l'efecte que té el grau d'entrecreuament de les matrius de suport en la colonització inicial d'aquestes cèl-lules.

Les xarxes copolimèriques basades en hialuronat i poli(acrilat d'etil) s'han desenvolupat amb l'objectiu d'obtenir nous materials semi-degradables, combinant les propietats biològiques del hialuronat i la bona compatibilitat del poli(acrilat d'etil) amb diferents cèl-lules, incloent les de l'entorn del sistema nerviós central. Per a la reacció de copolimerització va ser necessària una modificació prèvia de I 'hialuronat, determinant el seu grau de modificació. D'altra banda, les característiques antagòniques que tenen aquests dos materials permet el control de les propietats mecàniques i el grau d'inflat en entorns aquosos variant la composició de les xarxes copolimèriques. La composició experimental $i$ les propietats físiques $i$ mecàniques d'interès de les diferents composicions van ser determinades pels mètodes apropiats en cada cas. A més, es va estudiar la degradació enzimàtica de les xarxes copolimèriques, avaluant els fragments alliberats així com la integritat del material, amb la finalitat de garantir l'estabilitat del mateix durant un hipotètic procés regeneratiu. D'altra banda, la viabilitat cel.lular d'aquests materials va ser avaluada d'acord amb els possibles efectes citotòxics i inflamatoris sobre cèl-lules de l'entorn del sistema nerviós central. A més, la colonització d'aquestes cèl-lules sobre les xarxes copolimèriques va ser avaluada en estudis preliminars. 


\section{SUMMARY}

Unlike other tissues, the regeneration in the central nervous system during adulthood is limited due to the lack of neurogenic niches and the toxic microenvironment after an injury or degenerative disease. For this reason, the loss of neuronal populations and axonal connexions results in most cases irreversible. Nowadays, there are therapies focused on the regeneration of the central nervous system after an injury or disease, and they consist in cell implantation, drug delivery and electrical stimulation using electrodes. Despite these methods have shown some improvements in the regeneration and the recovery of the loss functions, they do not produce complete recovery of the loss functions or are transient. In the present work, the efforts have been focused on the development of structured materials (scaffolds) to provide a support for the different cells from the central nervous system, especially, from the brain tissue after stroke or similar damages. Besides, these materials could act as vehicles for cells being a neuronal source and/or growth factors providing substances to create a more permissive environment for axon regrowth.

In this work, the scaffolds are mainly composed of the sodium salt of hyaluronic acid, hyaluronan. This material has been chosen because of its natural origin in many tissues, including the brain one. Besides, some formulations based on hyaluronan and derivatives thereof are currently employed in clinical applications, and they have shown good biocompatibility and similar properties to the brain tissue. However, the unmodified hyaluronan have shown short residence times in the physiological environment because of its high solubility and fast degradation rate, lack of the control over mechanical properties, high swelling degree and hard handling to develop structured materials. In the present work, in order to develop stable and structured hydrogels, the hyaluronan has been modified following two strategies: crosslinking with divinyl sulfone, and the copolymerization of a hyaluronan derivative with ethyl acrylate to develop semidegradable copolymer networks.

The crosslinking of hyaluronan with divinyl sulfone has been characterized by physical, mechanical and chemical methods with the aim of determining the effect of the degree of crosslinking on the properties of the hydrogels. This chemistry has been employed to develop porous scaffolds by a repetitive method. Besides, the posterior treatments after the crosslinking reaction have been evaluated in terms of their effect on the enzymatic degradation. On the other hand, the cytotoxic and inflammatory effects of these materials on different types of cells from the central nervous system environment (astrocytes, neurons and endothelial cells) have been studied. Moreover, the influence of the degree of crosslinking on the initial colonization of these cells has been evaluated. 
The copolymer networks based on hyaluronan and poly(ethyl acrylate) have been synthesized with the aim of developing novel semi-degradable materials, which combine the biological properties of hyaluronan and good compatibility of poly(ethyl acrylate) with different cells, including those of the central nervous system. However, the use of hyaluronan in polymerization reactions required its previous modification, and hence the degree of modification was determined after synthetizing the hyaluronan derivative. Moreover, the opposite properties of both materials permit to obtain copolymer networks with tuneable mechanical properties and swelling degree in aqueous environments. The experimental composition of each component, as well as some physical and mechanical properties were determined by the appropriate methods in each case. Besides, the enzymatic degradation of the copolymer networks was evaluated, determining the scissed fragments and the structural stability of the different materials with the aim to guarantee the integrity of the materials through a hypothetical regenerative process. On the other hand, the cytotoxic and inflammatory effects of these materials on cells from the central nervous system environment were evaluated. Additionally, preliminary studies of cell colonization on the copolymer networks were performed. 


\section{TABLE OF CONTENTS}

CHAPTER I. INTRODUCTION ......................................... 17 -

1.1 Tissue engineering in the central nervous system ....................... 19 -

1.1.1 Problems of regeneration in the central nervous system ............ 20 -

1.1.2 Damages and diseases in the central nervous system ............... 22 -

1.1.3 Cell therapy .............................................................. 23 -

1.1.4 Biomaterials in central nervous system regeneration ................ 25 -

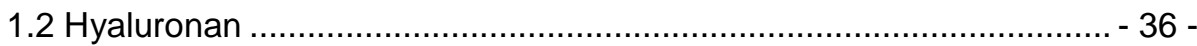

1.2.1 Sources and structure ............................................... 36 -

1.2.2 Rheology of hyaluronan solutions ...................................... 38 -

1.2.3 Degradation ............................................................... 40 -

1.2.4 Biodegradation ........................................................ 44 -

1.2 .5 Biological properties .................................................. 46 -

1.2.6 Chemical modifications............................................... 51 -

1.2.7 Medical applications of hyaluronan and its derivatives ............... - 62 -

1.2.8 Engineering of hyaluronan-based scaffolds ............................ - 64 -

1.2.9 Hyaluronan-based materials for CNS regeneration ................... - 71 -

1.3 Poly(methacrylates) and poly(acrylates) ................................. 73 -

1.3.1 Poly(methacrylates) and poly(acrylates) in tissue engineering .... - 75 -

1.3.2 Poly(methacrylates) and poly(acrylates) in central nervous system regeneration............................................................................ 76 -

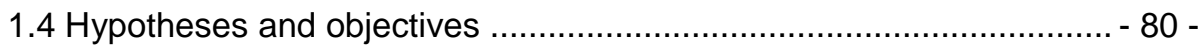

1.4.1 Hypotheses............................................................. 80 -

1.4.2 Objectives ................................................................... 80 -

CHAPTER II. MATERIALS AND METHODS........................ 83 -

2.1 Materials and cell lines ........................................................... $85-$

2.1.1 Polymers and monomers................................................ - 85 -

2.1.2 Crosslinking agents .................................................... - 86 -

2.1 .3 Initiator .................................................................... 86 - 


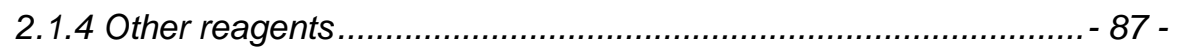

2.1.5 Cell lines and culture media ................................................... 87 -

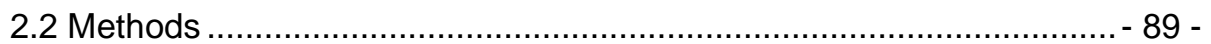

2.2.1 Crosslinking of hyaluronan polymer networks............................ 89 -

2.2.2 Preparation of crosslinked hyaluronan scaffolds .......................... 90 -

2.2.3 Synthesis of methacrylated hyaluronan....................................... 92 -

2.2.4 Synthesis of poly(methacrylated hyaluronan-co-ethyl acrylate) copolymer networks ................................................................... 92 -

2.3 Material characterization ........................................................... 94 -

2.4 Preparation of materials for cultures and viability assays ................. - 102 -

2.5 Colonization studies on scaffolds .................................................... 105 -

CHAPTER III. RESULTS....................................................... 109 -

3.1 Crosslinking of hyaluronan with divinyl sulfone ............................... - 111 -

3.1.1 Crosslinking of hyaluronan with divinyl sulfone ......................... 111 -

3.1.2 Characterization of crosslinked hyaluronan .............................. - 113 -

3.2 Crosslinked hyaluronan scaffolds ................................................... 137 -

3.2.1 Preparation of scaffolds ........................................................... 137

3.2.2 Characterization of scaffolds .................................................. 140 -

3.3 Poly(methacrylated-co-ethyl acrylate) copolymer networks .............. - 151 -

3.3.1 Methacrylated hyaluronan macromer ..................................... - 151 -

3.3.2 Synthesis of poly(methacrylated-co-ethyl acrylate) copolymer

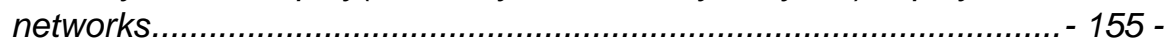

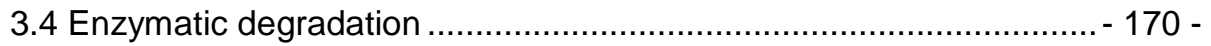

3.4.1 Determination of weight loss ................................................ 170 -

3.4.2 Determination of equilibrium water content .............................. 172 -

3.4.3 Morphological characterization ................................................ - 174 -

3.4.4 Determination of by-products ............................................ 179 -

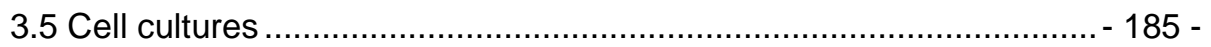

3.5.1 Viability assays .................................................................. - 185 -

3.5.2 Cultures of cell populations on scaffolds ................................ - 189 - 
4.1 Crosslinking of hyaluronan with divinyl sulfone ........................... $201-$

4.2 Crosslinked hyaluronan scaffolds............................................... 209 -

4.3 Poly(MeHA-co-EA) copolymer networks ................................ - 213 -

4.4 Enzymatic degradation ....................................................... 224 -

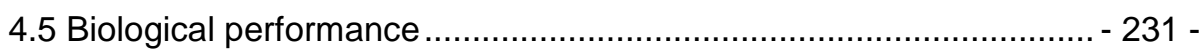

CHAPTER V. CONCLUSIONS ….......................................... 237 -

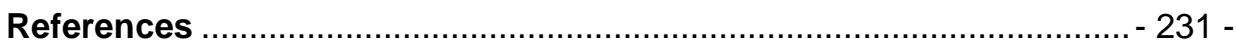

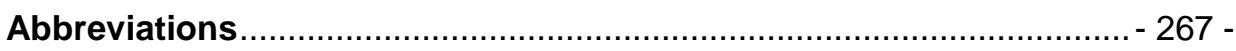



CHAPTER I

INTRODUCTION 



\subsection{Tissue engineering in the central nervous system}

Tissue engineering is an emerging and rapidly growing field with potential applications for tissue repair. It is an interdisciplinary field including advanced material science, cell biology, biochemistry, engineering and medicine. The US National Institutes of Health $(\mathrm{NIH})$ defines tissue engineering as:

Regenerative medicine/tissue engineering is a rapidly growing multidisciplinary field involving the life, physical and engineering sciences that seeks to develop functional cell tissue, and organ substitutes to repair, replace or enhance biological function that has been lost due to congenital abnormalities, injury, disease or ageing.

Many researchers have focused on addressing this novel area because of its wide range of applications in medicine. The aim of tissue engineering is to replace or restore the structure and function of injured or diseased tissues and organs. The normal function of an organ or tissue in the body is regulated by the activity of cells, extracellular matrix (ECM)-cells interactions, molecular signalling and other stimuli (mechanical, electrical...). Thereby, tissue engineering strategies include and combine cells, tissues, engineering biomaterials, biologically active molecules and stimulus. Therefore, tissue engineering is focused on the understanding of normal and pathological biological processes with the aim to recover the normal biological functions of damaged organs and tissues.

Nowadays, tissue engineering is applied in a broad range of diseases and damages of several tissues, even those affecting the central nervous system (CNS). Additionally, degenerative neuronal disorders and traumatic injuries into the CNS may cause severe and irreversible damage. Nowadays there are few clinical therapies to treat the neuronal disorders, and they achieve a modest functional recovery. Most clinical therapies are based on drug administration for neurodegenerative diseases with well-defined targets, such as Parkinson's disease (PD). However, there is no effective treatment in traumatic injuries, including traumatic brain injury (TBI), spinal cord injury $(\mathrm{SCl})$ and stroke, to restore lost functions and the clinical approaches are symptomatic and based on minimizing the damage progression. In patients with no effective drug administration, clinical therapies based on electrical stimulation can be employed, making use of implantable electrodes to stimulate axon growth. On the whole, the limitations of current therapies underline the need to search for novel strategies. 


\subsubsection{Problems of regeneration in the central nervous system}

Regeneration in the adult CNS is limited compared with other tissues [1]; the regenerative response is different even between the peripheral nervous system (PNS) and CNS. Regeneration in the PNS is more successful due to the presence of Schwann cells (SCs), which provide nutrients, guide and myelinate the peripheral axons and synthesize growth factors. Therefore, in the PNS exists a unique structure, the bands of Büngner consisting in clusters of oriented SCs, whose processes remain after axon injury and promote axon regeneration.

The limitation of regenerating the CNS is due to the lack of proliferation and regeneration of neurons and the inhibitory microenvironment for axon outgrowth. Although the developing CNS lacks of inhibitory effects on axon outgrowth, the predominant cells in the mature one are astrocytes and oligodendrocytes, which provide an inhibitory microenvironment for axon growth. The principal function of oligodendrocytes consists in supplying nutrients to neurons, but they express inhibitory proteins of axon growth as the myelin associated glycoproteins (MAGs) and tenascin R. Moreover, after CNS injury, astrocytes are activated to hypertrophic astrocytes, which are the principal cells of the glial scar. Hypertrophic astrocytes are clustered in layers giving a physical barrier for axon regrowth, and these cells also overexpress inhibitory molecules of axon growth, such as sulphated proteoglycans, MAGs and Nogo...[2]. 


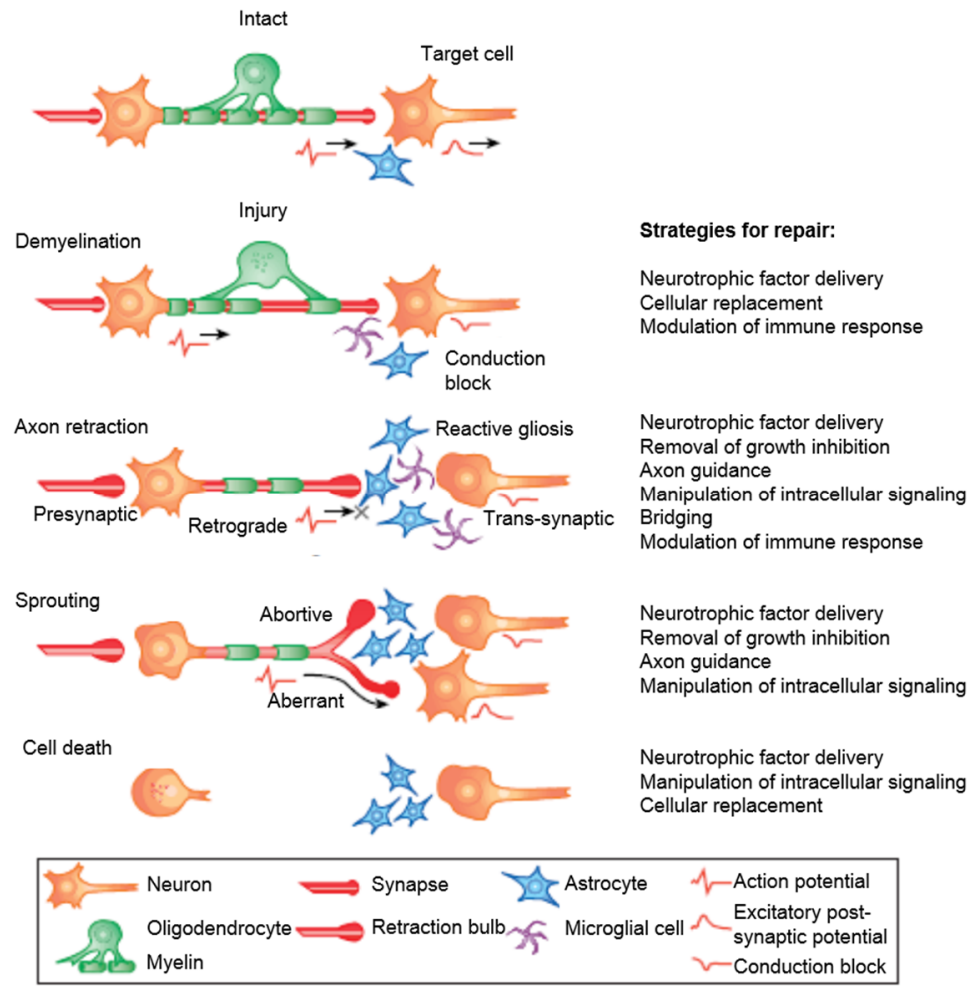

Figure 1. 1. Consequences of neural injury and strategies for repair [3].

In the case of traumatic injuries as $\mathrm{TBI}$ and $\mathrm{SCI}$, the primary damage generates an abrupt loss of cells and may result in the disruption of the blood brain barrier (BBB) and the blood spinal cord barrier (BSCB), respectively. The breach in the BBB and BSCB permits infiltration of foreign cells such as macrophages and fibroblasts and inflammatory molecules activating hypertrophic astrocytes. The injury in neurodegenerative diseases lacks of an abrupt primary loss of neurons but the toxic microenvironment leads to a progressive neurodegeneration. The neuronal injury and the inhibitory microenvironment result in degeneration on a cellular level: demyelination, axonal retraction, aberrant axonal pathfinding and cell death. Tissue engineering strategies for repair the injured CNS take into account these mechanisms of cellular degeneration and loss with the aim to accomplish functional regeneration (see Figure 1. 1) [3]. 


\subsubsection{Damages and diseases in the central nervous system}

Despite the wide variety of damages and diseases affecting CNS, they may be classified in two groups depending of their initial impact and progression. TBI and similar brain damages, such as stroke and hypoxia, and $\mathrm{SCl}$ are characterized by an abrupt damage and great tissue loss at the initial steps. However, neurological disorders, such as PD, Huntington's disease (HD), Alzheimer... show a progressive loss of neuronal subtypes at specific tissue regions, and many of these diseases ultimately lead to a loss of the patient autonomy. Moreover, pathology, damage progression and symptomatology are quite different between different neurological disorders. Below are described some habitual neurological diseases affecting humans [4].

In early stages of an abrupt brain or spinal cord injury, the damaged tissue presents necrosis due to physical trauma, haemorrhage and disruption of the blood brain barrier (BBB) or blood spinal cord barrier (BSCB), respectively, leading to ischemic processes. This disruption produces the infiltration of foreign components including fibroblasts, macrophages and cytokines, which are implied in the activation of astrocytes leading to the formation of the glial scar and inflammatory processes. After a few days, the apoptotic pathways are activated by the presence of a toxic microenvironment, giving a loss of glial cells and neurons without the possibility of replacement because of the absence of sufficient endogenous progenitors. Moreover, the microenvironment generated is inhibitory for axon regrowth giving loss of functions. Summarizing, the inhibitory nature of the CNS, due to the presence of astrocytes and oligodendrocytes, the lack of neurogenesis niches and the toxic microenvironment after injury avoid the regeneration in the brain and spinal cord.

$\mathrm{PD}$ is a neurodegenerative disease affecting around $1 \%$ of the population older than 60 years. It is caused by several factors but researchers have identified six gene mutations associated with this incidence, encoding $\alpha$-synuclein, parkin, $\mathrm{UCH}-\mathrm{LI}$, PINK-1, DJ-1, and LRRK2/dardarin. PD pathology is characterized by the loss of dopaminergic neurons in the substantia nigra within the nigrostriatal tract. For this reason, the striatum reduces dopamine levels and neuronal projections. In addition, other aspect of PD pathology is the generation of abnormal intraneuronal aggregates of protein, especially $\alpha$-synuclein, which are known as Lewy bodies. Further stages involve degeneration in the cerebral cortex and in the autonomic nervous system. The manifestation of PD includes tremor, bradykinesia, rigidity, cognitive decline and finally death. The animal model commonly employed to study the PD is obtained by administration of 6hydroxidopamine, which causes the lesion of dopaminergic pathways. In the initial stages of disease, L-DOPA administration is the most potent therapy to improve motor functions. However, this treatment becomes less effective in further stages, making necessary to find new therapies. 
$\mathrm{HD}$ is a dominantly inherited neurodegenerative disease that results from the expansion of the polyglutamine expansion in the gene encoding the huntingtin protein. This disease is characterized by the loss of brain striatal GABAergic neurons, and the degenerative process progressively extends to other brain regions including the cortex. The pathogenesis of $\mathrm{HD}$ and the function of huntingtin are not well understood, but this pathology is characterized by the loss of medium spiny neurons in the striatum and loss of neurons in the cortex leading to movement disorders (chorea), dementia, and eventually death.

Alzheimer's disease is characterized by the formation of neurofibrillary tangles in multiple areas of brain. Despite the initial stages of the disease remain unclear; the formation of neurofibrillary tangles is produced by the hyper-phosphorylation of the protein tau leading to neuronal loss. Early stages of the disease are characterized by memory impairment, and later stages affect other functions with a final loss of autonomy.

The amyotrophic lateral sclerosis (ALS) is a neurological disorder affecting motor neurons, producing a progressive degeneration of cortical and spinal neurons. Pathology of ALS is not well understood but it seems that the reason of the disorder lies on the alteration of the redox regulation both in neurons and astrocytes. The symptomatology includes alteration of motor functions, both involuntary and voluntary movements.

\subsubsection{Cell therapy}

Despite the discovery of neurogenesis in the CNS, an important problem of CNS injuries is that these processes cannot overcome massive cell death. Cell therapy tries to supply the injured CNS with neurons, glial cells, extracellular matrix components, adhesion molecules and neurotrophic factors. In general, implanted cells may provide a neuroprotective effect, remodel glial and neuronal cells, promote angiogenesis and synaptogenesis and modulate the inflammatory response. Several authors have employed a wide range of cells to help regeneration in the injured CNS: glial cells, adult stem cells (neural stem cells (NSC), mesenchymal and adipose tissue derived stromal cells), embryonic stem cells (ESCs) and induced pluripotent stem cells (iPSs) [5].

One of the most commonly studied cell types in the CNS regeneration are glial cells because of their ability to support axon outgrowth and release neurotrophic factors. Due to their role in axon regeneration in the PNS, SCs are widely employed in preclinical studies of $\mathrm{SCl}$, even some clinical trials have been reported [6]. SCs are cells, which promote regeneration in the PNS due to their ability to establish a permissive environment for axon regeneration and to provide neuroprotection. They produce growth and neurotrophic factors, secrete proteins 
stimulating axon growth, remove myelin debris following damage, supply myelin and provide pathways for regenerating axons. The implantation of SCs in the damaged spinal cord has demonstrated ability to promote axonal regeneration and myelination and a modest functional recovery [7]. However, the use of SCs in CNS regeneration is limited because of their ability to activate astrocytes contributing to the formation of glial scar [8].

Glial cells from the CNS including astrocytes, oligodendrocytes and microglia have been also studied in connection with CNS regeneration. However, the process of regeneration by macrophages is unclear and they can inhibit axonal growth after $\mathrm{SCl}$ [9] and their action could be limited to the elimination of myelin debris [10]. On the other hand, transplantation of glial cells, including astrocytes and oligodendrocytes, has been addressed by implantation in $\mathrm{SCl}$ models of neural progenitors restricted to differentiate into glial lineages. These cells have shown some benefits associated to their role in providing neuroprotection and remyelination and less glial scar formation than SCs [11]. However, only moderate functional recovery has been observed when neural progenitors are differentiated both, neural and glial cells lineages.

Olfactory ensheathing cells (OECs) constitute an interesting type of glial cells in CNS regeneration strategies, because of their phenotypic similarities to SCs [12], astrocytes [13] and oligodendrocytes [14]. OECs, in their natural environment, support axons from the PNS to the CNS and do not produce unfavourable interactions with the glial scar components. One shortcoming of these cells may be their inability to remyelinate axons, although this point has been debated. Some studies have demonstrated partial functional recovery when these cells are implanted in the injured spinal cord of paralyzed dogs [15], in the injured spinal cord of rats with respiratory deficits [16] and in the ischemic brain of rats [17]. Moreover, OECs have shown good results in some controversial clinical studies in stroke and $\mathrm{SCl}$ but they require further studies [18].

Adult stem cells from different origins (bone marrow, adipose tissue derived and NSCs) have been transplanted in several traumatic injuries and neurodegenerative diseases in preclinical studies, and in clinical trials of stroke [19] and $\mathrm{SCl}$ [20]. The main property exploited in mesenchymal and adipose tissue derived stem cells is their ability to differentiate into cells secreting neurotrophic factors, while neural stem cells can differentiate in both glial cells and neurons [21]. Other types of adult cells such as the adrenal chromaffin cells [22] and human retinal pigment epithelium cells (hRPEs) [23] have been employed in clinical trials to restore functions in PD, since they can be employed as suppliers of L-DOPA in situ. Both adrenal chromaffin cells and hRPEs have demonstrated modest functional improvements. However, these cells show a poor survival and only a small fraction is able to secrete L-dopa or dopamine, or is secreted in small amounts. 
ESCs [24] and iPSs [25] are emergent sources of cells with many possibilities in CNS regeneration. Their use, however, is limited by ethical considerations in the case of embryonic stem cells, and by the unsolved problem of teratoma formation in the case of iPSs.

Preclinical and clinical trials have shown that cells provide transient benefits to CNS regeneration based on paracrine effects. However, pure cell supply therapy has produced only modest results lagging behind initial expectations, due probably to different factors such as a poor cell permanence in the implantation site and survival in an aggressive environment leading to an inappropriate axon reconnection and guidance.

\subsubsection{Biomaterials in central nervous system regeneration}

Biomaterials have been defined as substances other than drugs or foods employed in diagnostic and therapeutics of tissues, organs and biological functions. The biomaterials and the by-products derived during the degradation thereof must accomplish a general characteristic, biocompatibility, evoking a minimal inflammation and toxicity in the physiological environment. Currently, ceramic, metallic, polymeric and composite biomaterials are employed depending on their application. Additionally, the biomaterials for tissue engineering are natural or synthetic and can be developed as temporary (biodegradable biomaterials) or permanent (biostable biomaterials) structures.

Depending on the application, the biomaterials must grant some requisites: in the present work, polymeric materials have been developed as three-dimensional structures (scaffolds) with the aim to mimic the ECM of a target tissue, allowing cell support in the regenerating tissue. Scaffolds directly affect cells behaviour, in terms of their adhesion, proliferation, migration, differentiation, and maintenance of phenotype. Scaffolds requirements might be variable depending on the target tissue and pathological process, but biomaterial scaffolds for tissue engineering should gather some general criteria:

- They should support specific cells retaining their functions such as proliferation, differentiation, phenotype maintenance, migration...

- Scaffolds should be biocompatible without excessive immune response and cytotoxic effects. Biocompatibility should be satisfied by the biomaterial and, if it is the case, by its degradation products.

- In general, biodegradable materials are preferred to biostable ones. In any case, the mechanical integrity of the scaffolds should be maintained through all the regenerative process. 
- Biomaterials should permit nutrient diffusion and cell ingrowth in order to allow the viability of cells and regenerating tissue. For this reason, biomaterials are usually produced as hydrogels or highly porous scaffolds.

- Scaffolds design and properties should be tailored depending on the requirements of the implantation site. Whenever possible, scaffolds should ensure batch-to-batch reproducibility.

The use of scaffolds in tissue engineering covers a broad range of tissues and organs, including bone, cartilage, eye, nervous system, etc. In the case of neural tissue engineering, the biomaterial-based scaffolds are performed with the aim to guide and stimulate axon outgrowth and give a neuroprotective effect in the toxic microenvironment generated after injury. For many decades, researchers have investigated the cues implied in axon growth and guidance by several in vitro studies. These cues are related to substrates (structure, composition, mechanical properties...), physical stimuli (electrical signals and mechanical stretch), cells (glial and other cell types) and permissive and inhibitory molecules of axon growth (ECM components, neurotrophic factors...). Employing biomaterials and biohybrids (combination of biomaterials with therapeutic cells and/or therapeutic molecules) might help to supply the necessary cues for an appropriate neural regeneration. At same time, biomaterials have been engineered and implanted in animal models of different CNS diseases and injuries. However, due to the variety of pathologic processes after injury or disease and the heterogeneity of the CNS tissues, biomaterials for CNS regeneration are engineered in a different way depending on the target tissue (see Figure 1. 2).

In this section are collected the different in vitro strategies using biomaterials to study the cues affecting axon regrowth. Moreover, in vivo studies in animal models and clinical trials employing different biomaterials to address the most common CNS injuries and diseases are described. Special attention is focused on the biomaterials employed in the present work, hyaluronan and poly(ethyl acrylate), whose applications in CNS tissue engineering are widely described in their corresponding sections [26]. 


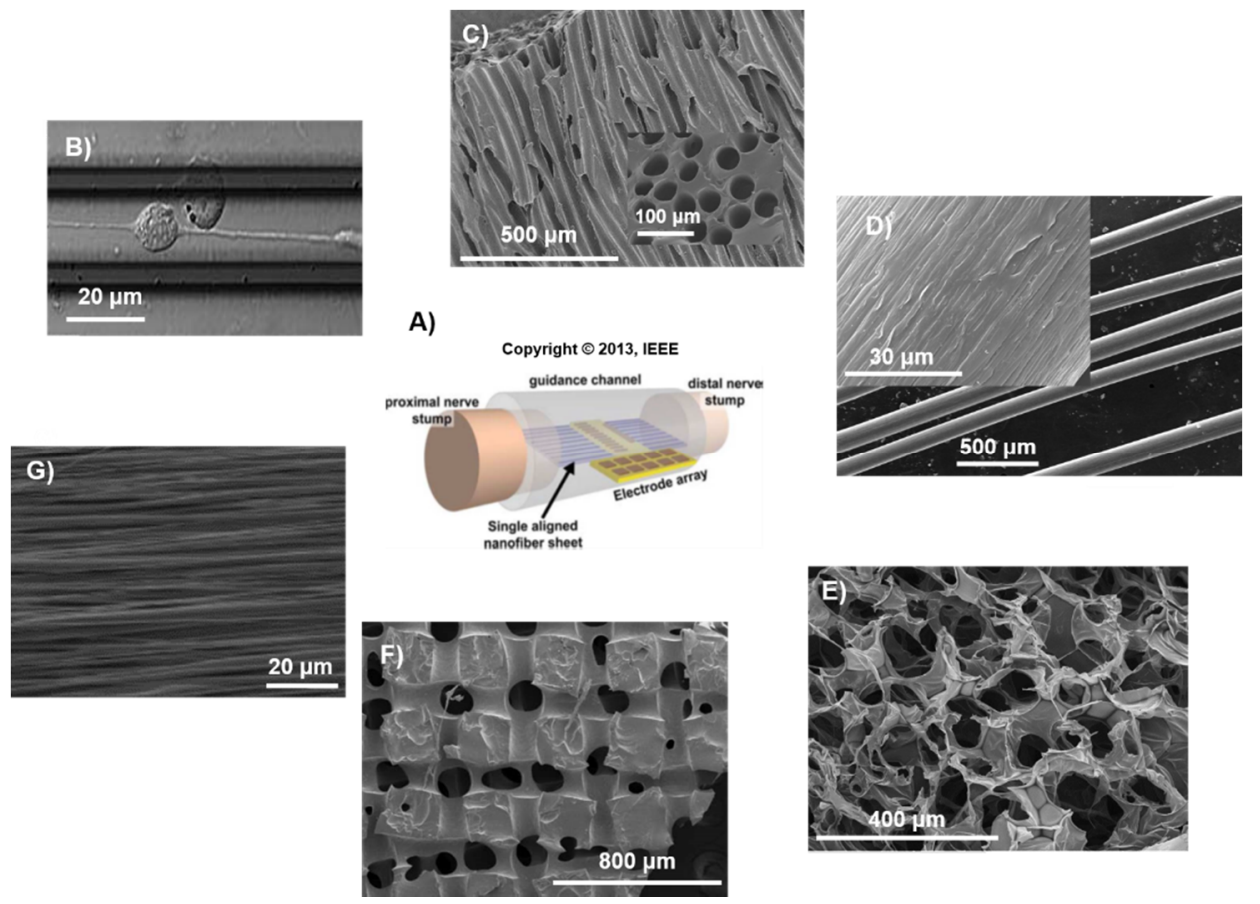

Figure 1. 2. Topographical motifs included in the biomaterials employed as scaffolds for CNS regeneration. A) Typical conduits based on an external channel and an inner matrix for peripheral nerve and spinal cord regeneration (from IP. Clements et al. [27] with authorization). B)

Micropatterned substrate (from WC. Huang et al. [28] with authorization). C) Multichannel conduit based on poly(ethyl acrylate), inset: detail of the cross-section (own our group). D) Microfilaments of poly( $\varepsilon$-caprolactone) obtained by micro-extrusion, inset shows a detail of the microfilament surface (own our group). E) Crosslinked hyaluronic acid scaffolds with interconnected porous structure (own our group). F) Poly(ethyl acrylate)-based scaffolds containing interwoven microchannels (own our group). G) Aligned electrospun fibres of poly(L-lactic acid) (from CC. Gertz et al. [29], with permission).

\subsubsection{In vitro studies using biomaterials}

\section{Topographical cues}

Cell differentiation and other cell processes are dependent of external stimuli and are well known that cell-substrate interaction and particularly topographical cues are important in this cellular behaviour. Bellamkonda discussed the anisotropy of both molecular and substrate topography as an important cue to obtain a faster and more orientated axon regeneration [30]. This idea has provided a wide variety of investigations, where researchers have studied axon outgrowth, both in vitro and in vivo, in anisotropic substrates such as surfaced patterns, fibres and conduits. These 3D structures with pronounced anisotropy have been obtained by different micro and nanofabrication techniques. 
Therefore, nano- and micropatterns have been fabricated by techniques such as lithography, photolithography, moulding templates, reactive ion etching, etc. Micropatterns are referred to scales above $1 \mu \mathrm{m}$ and their influence in cell behaviour occurs at a cellular level: their role in axon guidance is to support entire cells, both glia and neurons, and guide their cytoskeleton and processes [31-34]. Besides, nanopatterns present discrepancies in cell-substrate interactions since their small dimensions cannot harbour cell bodies and their influence is limited to neurites and to the assembly of ECM components and processes [35,36]. Despite the different effect of nano- and micropatterns on cell behaviour, authors suggest that their dimensions may influence neurite orientation, length and sprouting $[37,38]$.

One of the most common methods in order to obtain scaffolds with topographic cues is based on stacking layers of fibres. Nanofibrous scaffolds are usually obtained and aligned by electrospinning technique, while microfibers were produced by extrusion moulding with different morphologies. A wide range of synthetic and natural polymers have been electrospun to study axon outgrowth and guidance, such as aliphatic polyesters, polyamides, poly(methacrylates), polyacrylic acids, polydioxanone (PDS), collagen, gelatine, chitosan and hyaluronic acid... The natural ones show good biocompatibility and similar properties to neural tissues and the most employed synthetic biomaterials correspond to aliphatic polyesters including poly(lactic acid) (PLA), polyglycolic acid (PGA) and poly- $\varepsilon$-caprolactone (PCL) since they are biodegradable and some of them are approved by the Food and Drug Administration (FDA) for some clinical applications. Electrospun scaffolds have been employed as artificial matrices for neurons and glial cells, resulting in differentiation of NSCs and ESCs to neural lineages [39,40], and aligned fibres as substrates have resulted in neurite orientation and longer processes [41-43]. Micro fibres as substrate for axon and glia guidance constitute an unexplored field compared to nanofibres. However, some studies have demonstrated that synthetic microfibers are able to induce migration and orientation of glial and neural cells and promote axon extension [44-46].

Conduits have been widely researched for neural regeneration, especially focused on those cases where nervous fibres are coupled in bundles: peripheral nerves and spinal cord tracts. In vitro studies using conduits are usually carried out by assembly conduits containing inner structures, which confer to the substrates of anisotropy for glial cell migration and axon guidance. Habitually, the inner conduit is composed [47] or supports [48] electrospun fibres with the aim to provide topographical cues for glial and neural cells. These in vitro studies have shown that topography influences migration of cells and axon outgrowth, resulting in enhanced neurite lengths and migration in those using aligned fibrous meshes [48]. However, topographical cues can be accomplished using microfibers as 
inner meshes of conduits [44]. In this case, fibre diameter has shown to affect glia migration and neurite outgrowth. Even neurite growth can be faster than glia migration combining microfilaments with molecular cues, suggesting that a permissive environment allows axon ingrowth with independence of glia migration. Moreover, anisotropic conduits are accomplished using microchannel scaffolds, which are principally developed employing fibres templates, laser irradiation, freeze-drying, dipping and ion capillarity. Cultures of NSCs on microchannel conduits promote differentiation into glial and neural lineages, and some degree of infiltration of both types of cells has been observed within the channels [49]. Moreover, laminin (LN)-derived peptides (IKVAV and YIGSR) have been coupled on the inner surface of microchannels in order to address the synergetic effect of topographical and molecular cues [50] on cell colonization and neurite outgrowth compared to the separate effect of each one.

\section{Electrical stimulation}

Electrical stimulation of neural cells has led to the development of new frontiers for neural tissue engineering in the recent decades, including investigations of new conductive biomaterials and fabrication of electrodes to address several damages in the CNS. Currently, efforts have been focused on the study of conductive polymers because of some of them may act as the current organic polymers employed for tissue engineering. The electrical properties of these polymers are due to the presence of loosely held electrons in their backbones, which form conjugated $\pi$ bonds, and with the help of doping chemicals, the polymer can be charged negatively or positively. Traditionally, the employed dopant agents were inorganic compounds; however, the introduction of active biological dopants is more attractive from the point of view of biomedical applications [51]. The most commonly studied conductive polymers include polypirroles (PPy), polyaniline (PANI) and polythiophene derivatives, specially, the poly(3,4-ethylenedioxythiphene) (PEDOT). Other emergent polymers such as carbon nanotubes and nanofibres (CNTs and CNFs) [52] and piezoelectric polymeric materials $[53,54]$ are promising in developing new conductive polymers for neural tissue engineering. Several studies have shown that PPy is biocompatible with different cell types [55] and, particularly, with neural cells [56]. In recent years, PANI and PEDOT constitute and emerging growing field as conducting polymers for general tissue engineering applications. Recent studies have demonstrated the lack of cytotoxicity of PANI and PEDOT by in vitro studies, showing the biocompatibility of these alternative biomaterials [57,58]. Moreover, the tissue response to PPy, PANI and PEDOT substrates has been achieved in some preliminary in vivo studies, resulting in not toxic effects and an acceptable inflammatory response [59-61]. External electrical stimuli can be applied to a cell or tissue, producing an artificial action potential; this phenomenon is termed as 
electrical stimulation. Electrical stimulation can influence cellular activities such as proliferation, migration, differentiation, extension of processes and protein expression [62-64]. Therefore, electrical fields, both direct and alternating currents (DC and AC, respectively) can affect neuron activity. Some researchers have observed how an electrical stimulation influences the direction and extension of neurite outgrowth [59,65]; as well as, the alignment of other cellular types such as astrocytes [66]. In other studies, electrical stimulation has been found to increase neurite extension in differentiated PC-12 cells cultured on PPy films [55]. Most recent studies have corroborated the enhancement of neurite outgrowth with electrical stimulation in other cell types and different conductive biomaterials including NSCs on PANI $[67,68]$, cochlear neural explants on PPy [69] and dorsal root ganglia (DRG) explants on PPy [70]. Moreover, these studies have demonstrated, as Patel et al. [71] suggested, that one explanation for the increment in neurite lengths is due to the electrophoretic accumulation of proteins on the surface of the substrate.

\section{Mechanical stimuli}

The CNS is one of the softest tissues in the body, with elastic modulus between 0.1 and $10 \mathrm{kPa}$ [72]. Elasticity owns an important role at early stages of embryogenesis and development of tissues and, particularly in the CNS, since secretion of ECM components and migration of cells is influenced by mechanical cues. The rigidity of the brain increases with aging and the spinal cord shows the highest values of elastic modulus compared with other CNS tissues [73]. Therefore, the substrate rigidity may modulate the differentiation of neural precursors into glia or neurons. For example, ESCs differentiate into glia on softer substrates whereas to neurons in the more rigid ones [74], and neural progenitor cells (NPCs) and NSCs show a similar behaviour, differentiating preferably into neurons on softer substrates with modulus between those of the neonatal and the adult brain $[73,75]$.

In general, axon extension increases on softer substrates, so elastic biomaterials are more suitable to address axon regeneration. The most commonly employed substrates with modulated stiffness in neural regeneration are gels including hyaluronic acid, fibrin, hydroxyl poly(methacrylates), polyacrylamide and poly(ethylene glycol) (PEG) due to their similar mechanical properties to the CNS tissue. Similar trends in axonal extension and branching have been observed in different types of neurons, the softer or more elastic substrates generally increase neurite length, neuron attachment and branching compared to slightly stiffer ones $[74,76,77]$. However, axonal extension decreases in very soft substrates, when they present elastic moduli much lower than those of the CNS tissue [78,79]. Nevertheless, neurons have shown good attachment and large neurite extension 
on poly(dimethylsiloxane) (PDMS) substrates with elastic modulus higher than the CNS tissue [80]. Co-cultures of astrocytes and neurons on hydrogels with tuneable mechanical properties have shown that, contrary to neurites, astrocytes attachment decreases on soft substrates. This interesting result has highlighted the beneficial uses of soft substrates to address CNS injuries with presence of glial scar [74].

\section{Physical and chemical cues of substrates}

Some research are related to the study the effect on neural cells of many properties of biomaterials surfaces including charge, distribution of functional groups and wettability. Surface wettability can be modified by several techniques such as plasma polymerization, even gradients of wettability can be patterned on surfaces. Hippocampal neurons on substrates with wettability gradients have shown major cell density, longer processes and a faster development of longer processes than uniform substrates and hydrophobic regions [81]. The surface gradients seem to promote interactions between neighbouring cells and the development of neuronal networks. Similar results were found by culturing PC12 cells onto polyethylene surfaces with wettability gradients prepared by a corona discharge treatment [82]. Cell density was highest at intermediate wettability, around $55^{\circ}$, but the length of neurite processes increased on more hydrophilic regions, suggesting an important role of surface hydrophilicity on the differentiation of cells into neurons.

The functional groups of the biomaterials employed can affect cell attachment since surface charge, like wettability, is determinant on the biocompatibility of the biomaterials. Cells in general prefer to attach on positive surfaces because the residues of sialic acid from cell membranes produce a net negative charge. Moreover, hydrophilicity and hydrophobicity govern the formation of the nonspecific protein layer that can cause inflammation and the binding of other proteins involved in cell attachment [83].

\subsubsection{Biomaterials for brain injuries}

The justification for employing biomaterials for brain is principally due to the lack of cell support after injury, thus biomaterial scaffolds may mimic the natural environment of cells promoting their attachment and/or entrapment aiding the regenerative process. Scaffolds can lodge the cells in a 3D context, allow diffusion of nutrients and function as a substrate for cell survival, neurite extension, axon regeneration and cell infiltration. The selection of the biomaterials in brain regeneration is based on the principles to achieve neuroprotection of the surrounding native tissue avoiding the secondary cell 
death, and neuroregeneration of the damaged tissue to restore functions. From the biological point of view, a basic requirement of any material used is its biocompability, avoiding adverse cell response, such as formation of glial scar and/or acute inflammation. Besides, if possible, the biomaterial should be biodegrade without leaving any foreign residue as the regenerating tissue replaces it.

Structure size is an important aspect of the biomaterials because of invasive surgery can cause further tissue injury. Besides, biomaterial morphology is crucial to ensure cell attachment and colonization of the entire structure; thus, the structures employed should provide high surface/volume proportions (interconnected porous structures, microspheres, nanofibres...). Moreover, nutrient transport throughout the internal architecture of the scaffold and vascularization are also required for neurons survival. The preclinical strategies with biomaterials to address brain damages employ different structures based on these requirements for brain neuroprotection and neuroregeneration. Most of these structures include in situ formed gels [84] and porous interconnected scaffolds [85]. Furthermore, other types of structures such as fibres [86] and microchannels [87] have been also proposed. Normally, the use of natural polymers is preferable rather than synthetic ones because most of these biomaterials are present in the tissues and their effects are well known; many synthetic biomaterials have though a good integration with brain tissue and present some advantages respect to the natural ones, including lower costs, enhancement of mechanical properties, handling, etc.

\section{Natural biomaterials for brain regeneration}

Natural polymers have similarities with substances present in the tissues (polysaccharides and glycosaminoglycans (GAGs)), even many natural biomaterials form part of the ECM compounds such as collagen and hyaluronic acid. Thus, implanted devices based on natural polymers own in general higher biocompatibility with the host tissue. Moreover, in some cases, they may provide signals to cells improving cell-substrates interactions and modelling cell behaviour. They are usually biodegradable by enzymatic degradation, making them good candidates for implantable devices in brain. Most common natural polymers studied for brain implants include hyaluronic acid, collagen, chitosan, methylcellulose, other ECM compounds and peptides. One promising natural polymer for brain regeneration is methylcellulose since it has been approved by FDA in nerve repair. However, this material has been combined with other polymers in order to increase biodegradability [88]. Novel and promising in situ gels have been developed from self-assembling peptides nanofibres (SAPNs), which constitute peptides with the ability to form nanofibrous gels in contact with 
physiological ions [89]. Natural polymers are usually exploited by their ability to form in situ gels reducing invasive surgery, even they have been combined with NSCs [84], mesenchymal stem cells (MSCs) [90,91] and neurotrophic factors [92] increasing brain regeneration. In addition to good biocompatibility, employing natural biomaterials has resulted, in some cases, in a reduction of lesion volume, stimulation of growth factors production, promotion of neurogenesis, reduction of inflammatory response, neurite infiltration, stimulation of angiogenesis and slightly functional recovery [90-93]. However, materials such as chitosan, which possesses a positive charge, have demonstrated higher inflammatory response discouraging its use in brain regeneration [94].

\section{Synthetic biomaterials for brain regeneration}

The possibility to obtain a broad range of structures and properties using synthetic polymers has encouraged their use in brain regeneration. Therefore, biodegradable or biostable substrates with similar properties to those of neural tissue can be produced, mimicking the natural ECM. The most common synthetic materials in brain regeneration include aliphatic polyesters and poly(methacrylates), but other polymers have been investigated in order to address brain regeneration, such as poly carbonates, CNTs and silicon [95-97]. Employing aliphatic polyesters is attractive because of some of them are FDA approved for many medical applications. These materials have been used to develop several structures for brain regeneration, including electrospun nanofibres [86], porous scaffolds [98] and channels [87]. Aliphatic polyesters have shown good biocompatibility, reduction of secondary damages, neurite infiltration and low microglia and astrocyte activation. Silicon is commonly employed in neural interfaces electrodes [96] and CNTs require further studies in order to improve their biocompatibility with brain tissue [97].

\subsubsection{Biomaterials for spinal cord regeneration}

Despite most brain structures, axons within the spinal cord form longitudinal bundles, tracts, and they are similar to the nerve fascicles that compose the peripheral nerves. Thus, several researchers have proposed the use of nerve conduits for $\mathrm{SCl}$ because of their success in peripheral nerve repair. However, the spinal cord presents some physiological differences and the regeneration is more complex due to the limitation of regenerating the CNS. Owing to the complex geometries, the partial transection derived from spinal cord lesions and the need of invasive surgeries for the implantation of guidance conduits, many researchers have considered firstly the use of hydrogels. 
Scaffolds for spinal cord regeneration should include guiding structures for a correct axon regrowth, because the tracts in the spinal cord are clustered in aligned bundles of axons. Particularly, the tracts in the spinal cord have diameters about 100-1000 $\mu \mathrm{m}$, so the implanted scaffolds in the spinal cord must be permissive to the formation of axon bundles around these diameters. The swelling degree of a material under physiological conditions is important to maintain an appropriate alignment and avoid the compression of the regenerating tracts. Other properties are important to design scaffolds for spinal cord regeneration, including biocompatibility, controlled biodegradability, high surface/volume allowing cell attachment and the correct nutrient transport, similar mechanical properties to the host tissue, etc. The complex regeneration of the spinal cord forming aligned axons and tracts requires guidance structures, which can be fabricated by different techniques; the most common employed ones include rolled sheets of aligned electrospun fibres, freeze-drying, and moulding combined with particle leaching to generate porosity or by printing the polymers by CAD software.

\section{Natural biomaterials for spinal cord regeneration}

Natural polymers employed in spinal cord regeneration include fibrillary proteins, polysaccharides, GAGs and carbohydrates. The most common natural polymers employed include ECM components (collagen and hyaluronic acid) [99,100], polymers derived from algae (agarose and alginate) [101,102], and proteins derived from blood plasma (fibrin and fibronectin) [103,104]. They are potential materials for the purposes of spinal cord regeneration because of their possibilities in forming hydrogels in situ, avoiding invasive surgeries. However, in situ gels lack of effective axonal regrowth and guidance and scaffolds are commonly obtained as porous scaffolds and guidance conduits [105] to permit axon regeneration in an aligned manner. In addition to the current forms to obtain guidance conduits, some natural polymers can be submitted to a cooling gradient in a freeze-drying process [106] or ionic gelation leading to aligned microchannel conduits [107]. Collagen constitutes one of the most studied natural polymers for $\mathrm{SCl}$ since the FDA has approved some collagen-based conduits (NeuroMatrix ${ }^{\mathrm{TM}}$, Neuragen $\AA$ and Neuroflex ${ }^{\mathrm{TM}}$ ) for nerve regeneration. Employing SAPNs for $\mathrm{SCl}$ is an emergent and interesting method due to the possibility to avoid invasive surgery and generating fibrous matrices that might favour the correct axonal regrowth $[108,109]$. Implants for $\mathrm{SCl}$ based on natural polymers have shown, in general, good biocompatibility, axon regrowth, lack of excessive inflammation and slightly functional recovery. In addition, regeneration can be enhanced by combinational therapies including proteins, growth factors and neural stem cells in biomaterial matrices [101,110-112]. However, regeneration is not full achieved and only small gaps have been partially restored. 
Synthetic biomaterials for spinal cord regeneration

Most synthetic polymers permit design a broad range of structures for spinal cord repair, including conduits forming bridges between the rostral and caudal stumps. The most studied synthetic polymers for spinal cord tissue engineering include biodegradable ones, such as aliphatic polyesters (PLA, PGA, PCL, etc.), and biostable ones, such as methacrylate-based hydrogels including poly(hydroxyethyl methacrylate) (PHEMA) and poly(hydroxypropyl methacrylate) (PHPMA).

Aliphatic polyesters constitute promising polymers for spinal cord regeneration because of their biodegradability can be tailored by combining the different subtypes, even some of them are FDA approved as conduits for peripheral nerve regeneration: Neurotube $\AA$, which is composed of PGA, and Neurolac $\AA$, which is a copolymer of poly(DL-lactide- $\varepsilon$-caprolactone). PLA scaffolds have been designed obtaining different structures: aligned channels using a freeze-drying technique [113], porous interconnected scaffold [114] and conduits containing random and aligned electrospun fibres [115]. However, implantation of those structures in the injured spinal cord of rats has shown slightly regeneration just in the electrospun conduits. The inclusion of exogenous SCs expressing brainderived neurotrophic factor (BDNF) and neurotrophin-3 (NT-3) in PLA porous scaffolds resulted in a modest regeneration, but cell survival was low. On other hand, other problems associated with PLA conduits are referred to their structural stability through the regenerative process. Employing copolymers of poly(lacticco-glycolic acid) (PLGA) enhances the properties of implantable conduits and allows a better control over the degradation rate [116]. PLGA has been implanted principally as microchannel conduits, but microparticles have been injected in order to avoid invasive surgery [117]. PLGA conduits have demonstrated better results that those employing PLA regarding neuroprotection and neuroregeneration $[117,118]$. Moreover, PLA conduits have been combined with neurotrophic factors or SCs in order to improve regeneration and increase neuroprotective effect $[118,119]$. NSCs in combination with these conduits preferably to differentiate to neural lineages when they are implanted in rat models of SCI [120]. Other aliphatic polyester studied for spinal cord regeneration is PCL. Multichannel conduits have been developed from a PCL derivative, poly ( $\varepsilon$-caprolactone fumarate) (PCLF), for spinal cord regeneration [121]. PCLF conduits have resulted in major number of axons in the compared with those composed of PLGA. Moreover, PCL in combination with PLGA has been employed to obtain electrospun fibres conduits containing an internal matrix of based on SAPNs, RAD16-I, growth factors and neurotrophic factors to enhance axon attachment and motor functions of spinal cord [122]. 


\subsection{Hyaluronan}

\subsubsection{Sources and structure}

Hyaluronic acid was first isolated from vitreous humour from bovine eyes by Meyer \& Palmer in 1934 [123]. Hyaluronic acid is usually associated with the environmental cations and it is interchangeably referred as its sodium salt, hyaluronan (HA). HA isolation supposed the finding of a novel GAG, which is a family comprising other important molecules such as heparin, chondroitin sulphate, heparin sulphate... Over the following years, HA was found in several tissues of all the vertebrates. It is the major constituent in the ECM of some human tissues, such as the synovial joint fluid $(1400-3600 \mu \mathrm{g} / \mathrm{mL})$, the vitreous humour of the eye $(140-340 \mu \mathrm{g} / \mathrm{mL})$, cumulus cells around the oocyte prior to ovulation $(500 \mu \mathrm{g} / \mathrm{mL})$ and the umbilical cord $(4100 \mu \mathrm{g} / \mathrm{mL})$. However, HA is presented in a minor amount in other human tissues, such as dermis (200-500 $\mu \mathrm{g} / \mathrm{mL}$ ), epidermis $(100 \mu \mathrm{g} / \mathrm{mL})$, cartilage and CNS. Moreover, $\mathrm{HA}$ is produced in other animal species, such as rooster, whose combs contain the highest concentrations of HA ever reported for animal tissues $(7500 \mu \mathrm{g} / \mathrm{mL})$, and it can be produced by some bacteria, including Streptococcus equi zooepidemicus $[124,125]$. These both sources of HA are commonly employed in medicine for intra- and cutaneous applications.

The primary structure of $\mathrm{HA}$ is composed of two repeat units consisting in an uronic acid, D-glucuronic acid (GLCA), and an aminosugar, N-acetyl-Dglucosamine (GLCNAc), linked by alternating $\beta$ 1-3 glycosidic and $\beta$ 1-4 glycosidic bonds forming a lineal unbranched polymer (Figure 1. 3). HA constitutes a polyanion because of the carboxylic group from the GLCA unit and, at physiological conditions of $\mathrm{pH}$ and salt concentration, this group is widely hydrolyzed and balanced with the environmental cationic counterions, such as $\mathrm{Na}^{+}$. HA is a polymer with high molecular weight ranging between $200 \mathrm{kDa}$ and $10000 \mathrm{kDa}$ (where each disaccharide is approximately $400 \mathrm{Da}$, including sodium as counterion). Each polymeric chain is terminated by both reducing end and non-reducing ends. Reducing end is referred as the monosaccharide whose anomeric carbon (C1) is not involved in a glycosidic bond. The non-reducing end corresponds to the termination where $\mathrm{C} 1$ is joined by a glycosidic bond. The reducing end forms an equilibrium between the cyclic acetal and the opened aldehyde group, while the non-reducing end remains in the stable cyclic acetal form. 


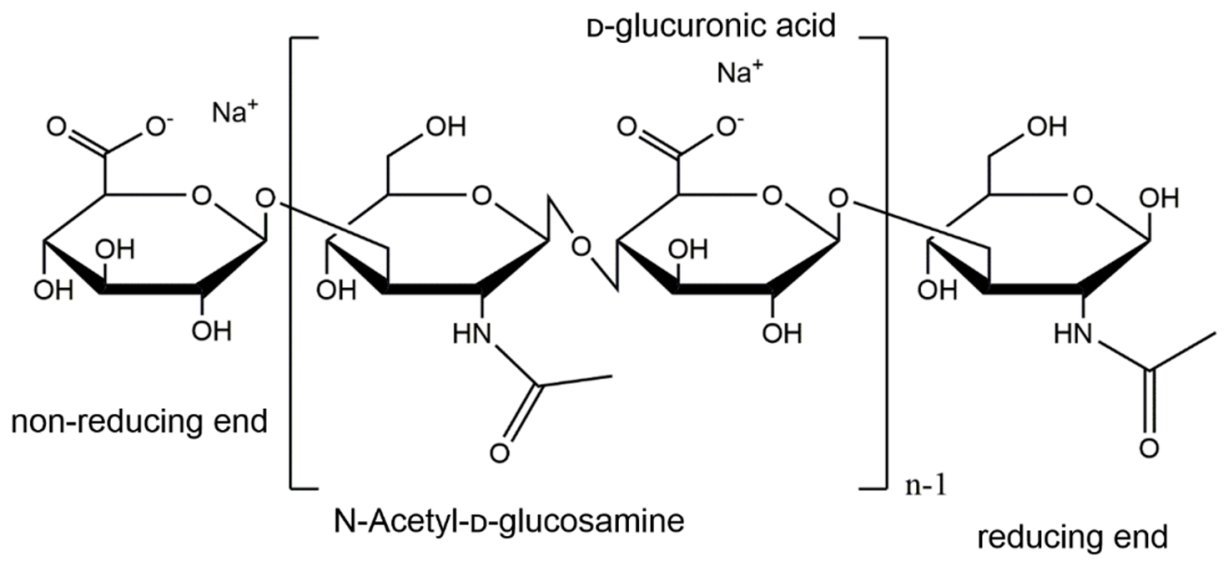

Figure 1. 3. Primary structure of hyaluronan including the reducing and non-reducing ends.

HA possesses singular properties, because of its polyanionic nature and high molecular weight, differentiating it from other linear unbranched polymers and affecting its secondary and tertiary structures. Assays using X-ray diffraction and Nuclear Magnetic Resonance (NMR) showed the presence of longer distances than the corresponding to $\mathrm{C}-\mathrm{C}, \mathrm{C}-\mathrm{O}$, etc. bonds, suggesting interchain interactions such as hydrogen bonds [126,127]. Therefore, the secondary structure is characterized by the relative rigidity of the glycosidic linkage and the intramolecular hydrogen bonds. These facts restrict the rotational freedom of the molecule affecting its conformation and impeding proteins and other globular molecules to diffuse through the HA matrix [128]. Moreover, other studies have demonstrated the presence of intramolecular and intermolecular hydrogen bonds [129]. These weak interactions allow multiple conformations of HA depending on the molecular arrangement and the nature of environmental cations: double helix, two antiparallel helices without coiling around each one, single helices in a tighter arrangement, six chains in an ordered arrangement, chains in a more compact arrangement and fully extended individual chains. Despite these conformations were found such a stable ones in the dry state, HA in water and under physiological conditions showed that intramolecular and intermolecular hydrogen bonds were formed and broken continuously giving transient networks [130]. This fact is due to energy differences of hydrogen bonds, whether HA is hydrogen bonded to itself or with water molecules. 


\subsubsection{Rheology of hyaluronan solutions}

The HA solutions contain unique properties due to singularity of HA molecules: polyanionic nature, common high molecular weight, relative rigidity of chains and the ability to form hydrogen bonds and other interactions. Thus, properties of aqueous solutions are strongly influenced by HA concentration and molecular weight, as well as the composition of the electrolyte $(\mathrm{pH}$, ionic strength and type of ions). In order to unify conditions and mask electrostatic repulsions, HA aqueous solutions are usually studied at neutral $\mathrm{pH}$ with ionic strengths up $0.1 \mathrm{M}$. Partial spatial volume of $\mathrm{HA}$ is about $0.56 \mathrm{~mL} / \mathrm{g}$ and the highest concentrations of HA employed in the studies are around weight fractions of $8 \%$. Under these conditions, the HA solutions are governed by hydrodynamic interactions and rheology is a potent method to determine their viscous properties. Rheology is carried out by subjecting HA solutions to different share rates and dynamic oscillatory conditions. These methodologies are employed in determining intrinsic and specific viscosity of HA and predicting solvent viscosity and molecular weight of chains.

Intrinsic viscosity [n] $(\mathrm{mL} / \mathrm{g})$ can be related with the molecular weight when HA is studied in dilute solutions and at very low or zero shear rates. Therefore, intrinsic viscosity has been fitted to two distinct regions; low and high molecular weight regions [131]. Solutions behaviours, at both regions, follow the Mark-HouwinkSakurada equation, $[\eta]=k \cdot M^{\alpha}$, where $M$ is the relative viscosity average molecular weight, and $k(\mathrm{~mL} / \mathrm{g})$ and $\alpha$ are constants with different values depending on the molecular weight region (Equation 1. 1).

$$
\begin{aligned}
& \text { a. }[\eta]=1.29 \times 10^{-3} \mathrm{M}^{1.056} \text { for } \mathrm{M}<10^{5} \\
& \text { b. }[\eta]=3.39 \times 10^{-2} \mathrm{M}^{0.778} \text { for } 10^{5}<M<10^{6} \\
& \text { c. }[\eta]=3.95 \times 10^{-1} \mathrm{M}^{0.604} \text { for } \mathrm{M}>10^{6}
\end{aligned}
$$

[Equation 1. 1]

Thus, $[\eta]$ is a measure of the hydrodynamic volume of the HA chains in solution, and hence its conformation. In the low molecular weight region, chains show a free-draining chain conformation, while they are in a ball-like configuration at higher molecular weights. Under similar shear rate conditions, it is possible to determine specific viscosity, $\eta_{\mathrm{sp}}$, as a simple function of the concentration and the relative viscosity average molecular weight, implicit in the [n] term, employing the Equation 1. 2 [132]:

$\eta_{\mathrm{sp}}=c[\eta]+k^{\prime}(c[\eta])^{2}+\frac{\left(k^{\prime}\right)^{2}}{2 !}(c[\eta])^{3}+\frac{\left(k^{\prime}\right)^{3}}{3 !}(c[\eta])^{4}$

[Equation 1. 2] 
where, the first term of the equation, $c[\eta]$, is the $\eta_{s p}$ at infinite dilution, while the entire right side of the equation is $c[n]$ multiplied by an expanded factor, $\left(k^{\prime} c[n]\right)$, truncated to include only the first four terms. The term $\mathrm{k}^{\prime} \mathrm{c} \cdot[\mathrm{n}]$ is a measure of the degree at which the domain of the coils overlaps in dilute and semi-dilute solutions. Thereby, the first two terms of Equation 1.2 are enough to predict $\eta_{\mathrm{sp}}$ in dilute solutions. However, higher concentrations require the four terms to consider intermolecular interactions. In Figure 1. 4 the dependence of $\eta_{\text {sp }}$ with $c[\eta]$ is fitted.

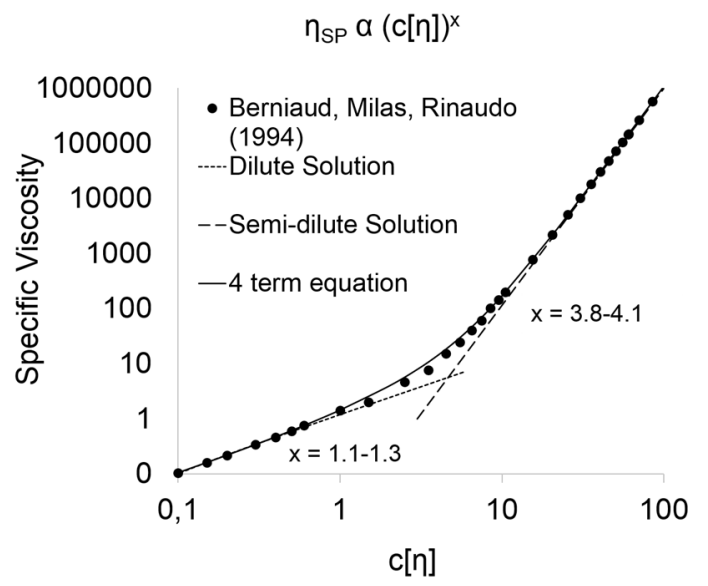

Figure 1. 4. Specific viscosity of hyaluronan as a function of the variable c.[n]. Modified from [130].

The models described above allow to study the viscosity of HA solutions under static conditions. However, viscosity of HA solutions depends on the applied shear rate because of chain entanglement. In order to investigate the influence of flow on solution properties, HA solutions have been measured at different shear rates and under dynamic oscillatory conditions [133,134]. Increasing flow rate leads to the measure of the shear rate at which viscosity drops (critical shear rate). This parameter is the inverse of the relaxing time, which is proportional to the solvent viscosity and the hydrodynamic volume in dilute solutions. On the other hand, dynamic oscillatory measures allow determination of changes in the storage elastic modulus, E', and viscous loss modulus, E'. At low frequencies, HA solutions behave as a viscous solution, while at higher frequencies they behave elastically. This inflection point is the critical shear rate, at which the curves of $E^{\prime}$ and $E^{\prime \prime}$ intersect. The transition from the viscous to the elastic behaviour of HA solutions is highly dependent on concentration and molecular weight; the higher the concentration and molecular weight, the earlier the transition occurs. 
HA solutions under the conditions described above are widely studied and show similarities with the physiological environment. However, other environmental conditions have been studied because of their interest in producing HA derivatives $[130,135]$. Thus, viscosity depends on temperature, the higher temperature, the lower intrinsic viscosity. This relation for dilute solutions with temperatures over the range $25-65^{\circ} \mathrm{C}$ can be explained using Equation 1.2. The addition of alkalis into the solutions leads to the reduction of viscosity because HA chains become more contracted, by eliminating intermolecular hydrogen bonds. Paradoxically, acidic conditions produce a reduction of viscosity due to the protonation of carboxyl groups and reduction of electrostatic repulsions since the HA possesses a pKa of 3.2 [130]. However, in the presence of salts this phenomenon is masked because of acidic conditions favour intermolecular hydrogen bond formation. Similarly, increasing ionic strength reduces viscosity by masking the electrostatic effect. However, depending on the type of counterion, viscosity is affected in a different way, for example HA chains in solutions at a constant ionic strength show similar hydrodynamic volume in solutions containing $\mathrm{Na}^{+}$or $\mathrm{K}^{+}$ions, but they are more contracted in electrolytes with $\mathrm{Ca}^{+2}$. Moreover, certain agents, including urea or ethanol, can disturb partially hydrogen bonds reducing viscosity of HA solutions. The effects on the conformation of the HA molecule due to the ionic strength and $\mathrm{pH}$ of the medium are difficult to compared from previous works because of the discrepancies in the methodologies employed. However, the hydrodynamic radius of HA dissolved in de-ionized water, $0.5 \mathrm{M} \mathrm{NaCl}$ and $0.5 \mathrm{M} \mathrm{NaOH}$ has been compared resulting in values of $95,33.5$ and $17.5 \mathrm{~nm}$, respectively [135]. The hydrodynamic radius is directly related with the gyration radius, and hence it results in a measure of the flexibility of the chains. In this case, the HA behaves as a semi-rigid molecule in de-ionized water (high hydrodynamic radius), whereas the chains become flexible when $\mathrm{NaCl}$ or $\mathrm{NaOH}$ is added. In addition, the discrepancies found between the hydrodynamic radius in $\mathrm{NaCl}$ and $\mathrm{NaOH}$ at similar concentration are only explicable by the disruption of hydrogen bonds in alkaline media.

\subsubsection{Degradation}

Fermented and extracted HA is, in general, a polydisperse polymer. Thus, it is usually characterized by its average molecular weight and polydispersity index. In order to determine chain sizes and dispersion, the authors have developed different techniques: viscosimetry and related methods (osmometry, sedimentation, diffusivity...); electrophoresis by exploiting its own polyanionic nature; and low-angle (LALS) and multi-angle light scattering (MALS) coupled to size exclusion chromatography (SEC) [136]. 
On the other hand, HA from healthy tissues, such as other physiological substances, suffers a catabolic pathway and is continuously synthesized, degraded to lower molecular weights and secreted out the body or employed in other processes. Thus, a group of enzymes, hyaluronidases (HYALs), degrades specifically HA and they are considered responsible for much of its catabolism. Despite HYALs are the principal field involved in HA cleavage, other agents can degrade the molecule including physical ones (irradiations, ultrasounds and highspeed stirring) and chemical reagents (acidic and alkaline media and free radicals). Thus, some of these agents are involved in the degradation of HA in some diseases and inflammatory processes, as well as in obtaining HA fragments. Degradation consists in the depolymerization of chains in smaller fragments, but this phenomenon sometimes takes place together chemical modifications within chains and monomeric units.

\section{Hydrolysis}

The hydrolysis of HA in aqueous solutions is spontaneously induced by the nucleophilic attack of water molecules within carbons involved in glycosidic bonds. The rate of hydrolysis is low under neutral $\mathrm{pH}$ and moderate temperatures, but the degradation is increased under acidic or basic conditions and higher temperatures $[137,138]$. Chain scission by hydrolysis occurs in a random fashion and it obeys to a first order kinetics $[139,140]$. However, there are significant differences in cleaving glycosidic bonds when performed under acidic or basic conditions: acids break glycosidic bonds in one step, whereas alkalis make it in two steps [138].

Cleavage of glycosidic bonds under acidic conditions involves three intermediate steps. First, a fast electrophile attack of protons leads to the protonation of both O-glycosidic atoms leading to an equilibrium between native and protonated forms of HA. Subsequently, take place the kinetic-determining step where the protonated form gives a carbonium-oxonium ion and a non-reducing end group. Finally, water molecules are fast added to the carbonium-oxonium ion leading to the formation of a reducing end group. Moreover, acids may perform a nucleophilic attack within O-1 atoms of glucuronic acid, opening rings and leading to chemical modified subunits. The authors suggest that acid hydrolysis cannot attack the $\mathrm{N}$-acetylglycosamine unit since the $\mathrm{N}$-acetyl group is protonated drawing electrons from the glucusodic oxygen.

Hydrolysis under basic conditions begins with a nucleophilick attack of water molecules and hydroxides within the $\mathrm{C}-1$ and $\mathrm{C}-2$ of the $\mathrm{N}$-acetylglucosamine subunit breaking the pyrose ring. A second nucleophilic attack of water and hydroxides within the oxygen atoms of $\beta(1-4)$ glycosidic bond and the $0-1$ from the $\mathrm{N}$-acetylglucosamine group generates two possible chain scissions. This 
process yields to chemical modifications of the native chains leading nonreducing end and ring-opened species. Moreover, basic media favours the equilibrium of the amino form of the $\mathrm{N}$-acetyl group. Under this form, hydroxides produce a nucleophilic attack to the carbon of the $\mathrm{N}$-acetyl group linked to the nitrogen atom generating deacetylation and producing a modified group with a primary amine.

Despite acid and basic hydrolysis produce a reduction of HA molecular weight, it is in a random fashion manner yielding a mixture of oligomers with high polydispersivity and chemical modification in the native subunits. These methods are also not employed to obtain fragments, because of the possible presence of undesirable products and needing to separate HA chains in monodisperse sizes.

The effect of temperature on hydrolysis can be explained by an Arrhenius-type relationship. Slow degradation rates were observed at temperatures in the range of $25-90 \stackrel{\circ}{ } \mathrm{C}$, while faster degradation was observed, even at short times, at temperatures around $130{ }^{\circ} \mathrm{C}[137,141]$. The combination of acidic and basic media with temperatures up to ambient ones produces faster kinetics of chain scission.

\section{Degradation using ultrasounds}

Ultrasonic degradation or sonication produces chain cleavage in a non-random fashion, leading to bimodal molecular weight distributions [142]. Moreover, the kinetic of depolymerization during sonication follows a linear relationship [143]. Ultrasonic degradation allows obtaining small HA fragments with molecular weights of few $\mathrm{kDa}$. However, complete degradation to monomeric units is not accomplished by this method, even employing longer times and higher energies. Sonication is an efficient method in order to reduce chain sizes to intermediate molecular weights without significant chemical modifications [144].

\section{Degradation by oxidants}

Oxidants, particularly reactive oxygen species (ROS), are important degrading agents of HA during different physiological processes. ROS in the body are generated from different sources including the activity of cells, enzymes, chemicals and physical irradiations. Degradation and synthesis of HA is in equilibrium in healthy tissues, whereas many diseases affecting synovial fluid in the joint, including osteoarthritis and rheumatoid arthritis, are characterized by high concentrations of ROS and a harmful action. Most typical ROS implied in HA molecular weight reduction include superoxide anion radicals, peroxides, hydroxyl radicals, singlet oxygen and nitric oxide [145]. 
The mechanism of degradation of HA chains is different depending of ROS chemical nature. In general, ROS randomly abstract a hydrogen atom from either HA subunits leading to the formation of macroradicals within chains, and subsequent electronic rearrangements give carbon-centred radicals in carbons implied into the glycosidic bonds. For example, hydroxyl radicals can subtract hydrogens from all the $\mathrm{C}-\mathrm{H}$ bonds in the glucuronic acid and at all sites, except the $\mathrm{N}$-acetyl group and $\mathrm{C}-2$, within the $\mathrm{N}$-acetylglucosamine subunit. Whereas, hypochlorous acid attacks the nitrogen atom of the $\mathrm{N}$-acetyl group and posterior electronic rearrangement leads to the formation of carbon-centred radicals at $\mathrm{C}$ 4 of the glucuronic acid unit, generating glycosidic bond scissions. Moreover, the reduction of molecular weight using ROS and other oxidant species is usually accompanied by chemical modifications of the fragments, such as oxidations, extraction of hydrogen atoms and scission of functional groups. These chemical modified species are those implied in inflammatory processes in diseases associated with reduction of molecular weight of HA by ROS [146,147].

\section{Degradation by physical irradiations}

Several authors have studied the effect of physical irradiations on HA degradation, including microwaves, UV rays and gamma-irradiations [148,149]. Understanding effect of irradiations on HA is important since most of them are usually employed in order to obtain sterilized (using gamma-irradiation) or disinfected (using UV) materials for clinical applications. The authors suggest that irradiations on HA act similarly to oxidant species. These methods induce radical and singlet oxygen formation with the ability to attack chains giving carboncentred radicals within carbon atoms involved in glycosidic bonds. Subsequently, these radicals are followed by cleavage of glycosidic linkages. Moreover, HA solutions and swollen hydrogels are in general more affected by irradiations, resulting in higher rates of chain scissions that those employing HA powder or dry hydrogels [150].

Depending on radiation nature, exposition times and dose, the degradation of $\mathrm{HA}$ is qualitatively and quantitatively different. Gamma-irradiation and $\mathrm{Hg}$ lamp irradiations (wavelengths up $297 \mathrm{~nm}$ ) give significant loss of molecular weight and chemical modifications within the monosaccharide units, even at low dose. On the other hand, degradation under microwaves and UV imply lower rates of reduction of molecular weight lacking significant chemical modifications. However, harsher conditions (acidic or alkaline $\mathrm{pH}$ ) may produce chemical modifications and increases degradation rates [151]. 


\subsubsection{Biodegradation}

HA is localized in the ECM of most tissues and it is principally present in its high molecular weight form in healthy tissues. HA is continuously synthesized and degraded, in order to maintain the equilibrium of its catabolism. The degradation is principally carried out by a group of enzymes, which were defined as hyaluronidases (HYALs) by Karl Meyer [152]. HA is present in eukaryotic and prokaryotic cells, hence its HYAL-mediated degradation occurs in large amount of organisms observing some differences [145,153], and it is increased in some pathological diseases, including the CNS damages [154]. In mammals, the degradation of hyaluronan may occur by two pathways: local digestion, where HA internalization and degradation is conducted by cells within the host tissues; and drainage into the vasculature and clearance in cells of the lymph nodes, liver and kidneys. In any case, the mechanism of degradation is similar beginning with the partial degradation of high molecular weight HA in the host tissues. Partially degraded chains can be internalized by receptor-mediated mechanism or endocytosis into the lysosomal compartment of cells and HA is fragmented in smaller oligosaccharides. Moreover, the organism usually secretes these fragments, but they are often degraded to monosaccharides by other types of enzymes, exoglycosidases, within the lysosomal compartments. Enzymatic degradation of $\mathrm{HA}$ is habitually performed at low $\mathrm{pH}$ (4-5.5), where HYAL activity is optimal. For this reason, most of the degradation studies in vitro are carried out at low $\mathrm{pH}$ using acetic buffers. However, some hyaluronidases from the eukaryotic organisms show activity in a broad range of $\mathrm{pH}$, even at neutral ones.

Biodegradation of HA requires several enzymes to cleave the larger chains till obtaining monosaccharides. However, HYALs are also referred to those enzymes able to cleave glycosic bonds of HA oligosaccharides and larger chains obtaining smaller fragments with disaccharides as repeat unit. Therefore $\beta$ exoglycosidases, which remove single sugar units from HA chains, are not considered as HYALs. The enzymes $\beta$-exoglycosidases are present in prokaryotic and eukaryotic cells and include $\beta$-exoglucuronidase and exo- $\beta-\mathrm{N}$ acetylglucosaminidase, which remove single glucuronic acid and $\mathrm{N}$ acetylgucosamine units, respectively. On the other hand, HYALs belong to the enzyme family $\beta$-endoglycosidases and are localized in bacterial and eukaryotic cells of many organisms. Depending on the mechanism to cleave the glycosidic bond HYALs are classified in three groups: microbial $\beta$-endoglycosidases, $\beta$ endoglucuronidases and endo- $\beta-\mathrm{N}$-acetylhexosaminidases. The last two groups are eukaryotic $\beta$-endoglycosidases. 
Prokaryotic $\beta$-endoglycosidases cleave randomly chains of HA and other GAGs by a lyase mechanism by the $\beta(1-4)$ glycosidic linkage $[155,156]$. Thus, degradation of chains is performed by $\beta$-elimination yielding tetra-and pentasaccharides with unsaturated groups. Hence, the action of these HYALs produces chemical modifications.

$\beta$-endoglucuronidases are present in eukaryotic organisms including certain annelids and crustaceans. These hyaluronidases produce random scission by a hydrolysis mechanism of the $\beta(1-3)$ glycosidic linkage. These enzymes cleave HA generating tetra- and hexa-saccharides with glucuronic acid at the reducing end. The mechanism of hydrolysis does not give chemical modifications.

Endo- $\beta$ - $\mathrm{N}$-acetylhexosaminidases are present in eukaryotic organisms of vertebrates including mammals. These hyaluronidases are localized in mammalian spermatozoa, lysosomal compartments and different venoms of reptile and insects. These enzymes cleave randomly HA chains by hydrolysis of $\beta(1-4)$ glycosidic linkage yielding oligosaccharides of different lengths with $N$ acetyl glucosamine at the reducing end. The mechanism of action has been widely studied and includes several intermediate stages, but it is partially understood [145]. First, the HA substrate and the HYAL are bonded and the carbonyl oxygen of the $\mathrm{N}$-acetyl group is positioned next to the $\beta(1-4)$ glycosidic bond. Subsequently, both groups form a covalent intermediate breaking the glycosidic bond. The glucuronic acid becomes protonated donating its $\mathrm{H}$ atom to the detached glycosidic oxygen. The intermediate covalent bond is also cleaved leading to the re-protonation of the glucuronic acid. Finally, the HA product is released from the hyaluronidase.

Mammalian species show different types of hyaluronidases; in mouse genome has been identified seven hyaluronidase-like gene sequences, while in humans have been found six sequences [157]. The hyaluronidase genes are known as hyaluronicglucosaminidase (Hyals) genes and they are localized in two different chromosomes in humans. The expression of these gene sequences gives three different types of HYALs named as Hyal1, Hyal2, Hyal3, Hyal4, Hyalp1 and PH20.

The most important and the major expressed HYALs in human somatic tissues are Hyal1 and Hyal2 [158]. Hyal1 was the first somatic hyaluronidase isolated from human plasma. It is a single polypeptide glycoprotein of $57 \mathrm{kDa}$ or $45 \mathrm{kDa}$ (in its processed form) [158]. Hyal2 is anchored to the plasma membrane and cleaves HA of high molecular weight to fragments around $20 \mathrm{kDa}$ [159]. While Hyal1 degrades HA of any chain size, yielding oligosaccharides, principally tetraand hexa- saccharides. Thus, these enzymes constitute the first degradation step of HA from the ECM. 
$\mathrm{PH}-20$ or SPAM-1 is known as testicular hyaluronidase because of its localization and important role in the fertilization process of mammals [160]. It is localized in the sperm surface an in the lysosome-derived acrosome, and its function is to degrade the HA-rich cumulus layer involving the egg. It is a polypeptide synthesized with a molecular weight of $64 \mathrm{kDa}$, and it is processed during maturation in two fragments linked by disulphide bridges with two terminal domains of 41-48 and $27 \mathrm{kDa}$, respectively. However, its presence in tissues with certain malignancies suggests that $\mathrm{PH}-20$ have other functions. $\mathrm{PH}-20$ yields to small oligosaccharides as the case of Hyal1 [161].

The other human HYALs (Hyal3, Hyal4 and Haylp1) are not well understood yet because of the difficulty in determining hydrolase activity. Hyal3 has been found in chondrocytes, testis and bone marrow and it is widely expressed during differentiation of fibroblast to chondrocytes [162]. Hyal3, as well as Hyal2, is upregulated by inflammatory cytokines including IL-1 and TNFa. Surprisingly, recent studies have shown Hyal4 has no evidence of hydrolytic activity on HA, exhibiting activity towards other GAGs such as chondroitin sulphate [163]. This enzyme has been localized in different tissues such as the placenta, skeletal muscle, and testis, suggesting that its activity is restricted to specific tissue functions. In a similar way, murine Hyalp1 has shown no hydrolytic activity on HA. This enzyme has been observed in mice testes and its functions may overlap to those HYALs involved in fecundation [164].

Unlike bacterial HYALs, determination of activity in hydrolase ones is by conventional methods. The lack of chemical modifications in degraded HA products, such as those produced by bacterial Hyal, impedes the activity measurement by spectrophotometry. Thus, the current methods in determining hydrolase activity are based on the measurement of the formed $\mathrm{N}$-acetyl glucosamine at the reducing end or by viscosity and turbidity methods.

\subsubsection{Biological properties}

\subsubsection{Biosynthesis}

The biosynthesis of $\mathrm{HA}$ in the mammals is regulated by three different transmembrane glycosytranferase isoenzymes, known as hyaluronan synthase (HAS1, HAS2 and HAS3) [165,166]. Their gene sequences are located in different chromosomes, but their amino acid sequences are identical at $50-71 \%$. However, each isoenzyme synthetize HA chains with different ranges of molecular weight. HAS3 synthetize HA of low molecular weights around 100$1000 \mathrm{kDa}$, while HAS1 and HAS2 produce HA in a broader range from $200 \mathrm{kDa}$ to $3.9 \mathrm{MDa}$ ). $\mathrm{HA}$ is present in all vertebrate tissues, but is especially abundant in connective tissues molecular weights between $800 \mathrm{kDa}$ and $10 \mathrm{MDa}$. 
Despite GAGs are usually synthesized within the Golgi apparatus, HA polymerization is performed on the plasma membrane. Synthesis of the saccharides taking part in the HA structure requires employing uridine diphosphate glucuronic acid (UDP-GIcUA) and uridine diphosphate $\mathrm{N}$ acetylglucosamine (UDP-GlcNAc) as substrates. HAS isoenzymes extrudes HA form the plasma membrane onto the cell surface or the ECM. These enzymes possess two components, one of them adds the glucuronic acid subunit and another one couples the $\mathrm{N}$-acetylglucosamine group, alternating the coupling of both saccharides.

\subsubsection{Hyaluronan-binding proteins}

HA chains possess certain binding sites with several cell receptors and ECM proteins. These proteins are known as hyaladherins and include intracellular receptors, both plasmatic and transmembrane ones, and extracellular proteins taking part in the ECM structure (see Figure 1. 5). HA have also two important functions mediated by binding proteins: structural and modulation of signalling pathways [167]. Some of the known HA-binding receptors include cell-surface glycoprotein 44 (CD44), receptor for hyaluronate-mediated motility (RHAMM), lymphatic vessel endothelial HA receptor (LYVE1), hyaluronan receptor for endocytosis (HARE), toll-like receptors (TLR-4/2), layilin and liver endothelial cell clearance receptor (LEC receptor). On the other hand, some of the extracellular hyaladherins include versican, aggrecan, fibrinogen and neurocan. Therefore, HA can bind to large amounts of ECM proteins forming aggregates and acting as a structural matrix $[168,169]$. Extracellular hyaladherins are HA-binding proteoglycans of the ECM in the different tissues: versican present in soft tissues, aggrecan and fibrinogen predominant in cartilage, neurocam and brevicam important in CNS, etc.

\section{Cell-surface glycoprotein CD44}

CD44 is the most common HA-binding receptor and it is localized on the membrane of all cell types. CD44 is a type I cell transmembrane glycoprotein that can interact with other molecules, including ECM proteins, growth factors and cytokines. This glycoprotein is expressed in different isoforms due to alternate splicing of 20 exons. In general, each isoform contains four domains, including intracellular, extracellular and transmembrane domains. Its affinity to bind HA is influenced by polypeptide sequence, glycosylation and oligomerization. The different isoforms show molecular weights in the range between $75-250 \mathrm{kDa}$. 
CD44 is overexpressed by cytokines such as IL-1 and TNFa and growth factors including the TGF- $\beta$ superfamily and endothelial growth factor (EGF). In some tissues, such as cartilage, the higher CD44 expression implies increasing the ability for HA binding, its internalization and degradation. CD44 up-regulation by IL-1 influences the proliferation of certain cell types including smooth muscle cells and may contribute to the development of pathological lesions. EGF is a growth factor that can stimulate over-expression of CD44 in fibroblasts, tumoral cells and epithelial cells. The production of bone morphogenetic protein (BMP), which belongs to the superfamily TGF- $\beta$, is also stimulated giving an osteoinductive effect [170].

In addition, CD44 can be associated with a large amount of ligands including fibroblast growth factor (FGF), osteopontin, matrix metalloproteases, kinases, GTPases, while HA can bind to other hyaladherins implied in the regulation of cell functions or taking part of the ECM [171]. Thereby, HA-CD44 interaction may function as a docking of proteins, growth factors and other molecules on cell membranes, acting as a co-receptor/co-regulator agent. Most specific cellular functions of the HA-CD44 binding include cell-cell aggregation, motility, adherence, proliferation, signal transduction, endocytosis and degradation of HA [172]. Thus cells enriched in HA have been found in processes where migrating and proliferating cells play important roles: embryonic development, wound healing, tumorogenesis and metastasis, vascularization...[171,173].

\section{Hyaluronan-mediated motility receptor (RHAMM) and Layilin receptor}

RHAMM is an extensively coiled-coil protein with a globular head with a molecular weight ranging from 59-80 kDa. Contrarially to other intracellular hyaladherins, RHAMM does not constitute a transmembrane protein and it is also present on cell surface, within the cytoplasm and in the nucleus. RHAMM can interact with several sulphated GAGs, as well as with biotinylated HA [174]. This receptor has been observed in cells such as fibroblasts, endothelial cells, smooth cells, macrophages and many types of tumoral cells [175]. RHAMM form association with actin and microtubule cytoskeleton, centrosomes and cell lamellae and other proteins associated with motility [176]. Thereby, RHAMM interacts with microtubule associated proteins (MAPs) from the cytoskeleton and transforming acidic coiled-coil domains (TACCs) involved in cell cycle and differentiation. Moreover, this receptor can modulate the extracellular signalling regulated by kinases showing a targeting action to specific substrates [177]. Particularly, HARHAMM binding has an important role in tissue repair, inflammation, proliferation and tumorogenesis $[178,179]$. 
Layilin is a type I transmembrane glycoprotein of $55 \mathrm{kDa}$ with similar sequence to C-selectin. However, Layilin domains have no homology with other known HA receptors $[180,181]$. This molecule is involved in the association between cell membrane and actin cytoskeleton. Thus, layilin plays an important role on adhesion, and hence affects migration and the mediation of signals form ECM.

Hyaluronan receptor for endocytosis (HARE) and lymphatic vessel endothelial receptor 1 (LYVE-1)

HARE is an integral membrane glycoprotein generated by the protolithic cleavage of stabilin 2 or FEEL2 and it has been identified two forms with different molecular weights: 175 and $300 \mathrm{kDa}$. This molecule is typical of the lymphatic endothelium, but it has been observed in endothelial cells of other tissues, such as brain, kidney, eye and heart [182]. HARE possesses several binding motifs, including $H A$, fascilin and EGF binding domains. The principal known function of HARE is its role in endocytosis and subsequent degradation of HA via catherin colocalization.

LYVE is a membrane glycoprotein similar to CD44 in structure and its function localization and functions is similar to HARE. Thereby, LYVE has been observed in lymphatic endothelium, endothelial cells of other tissues and macrophages and it is related to the internalization and degradation of $\mathrm{HA}$ [183].

\section{Toll-like receptors (TLRs)}

TLRs are receptors from the interleukin-1 receptor family. These transmembrane receptors are characterized by its very rapid response and they are broadly expressed in many tissues. TLRs are associated with the innate mature response, by activating dendritic cells, monocytes and macrophages [184-186]. TLR-2 can recognize mycobacteria and Gramm positive bacteria, while TLR-4 are activated by the inflammatory molecule, lipopolysaccharide (LPS) [187].

The role of the HA-TLR binding is size-dependent: HA of high molecular weight induces the expression of kinases, which interrupt TLR signalling, while oligosaccharides induce TLR signalling regulated by NF-kB and AP1 pathways, which are activated by chemokines and cytokines [188]. 


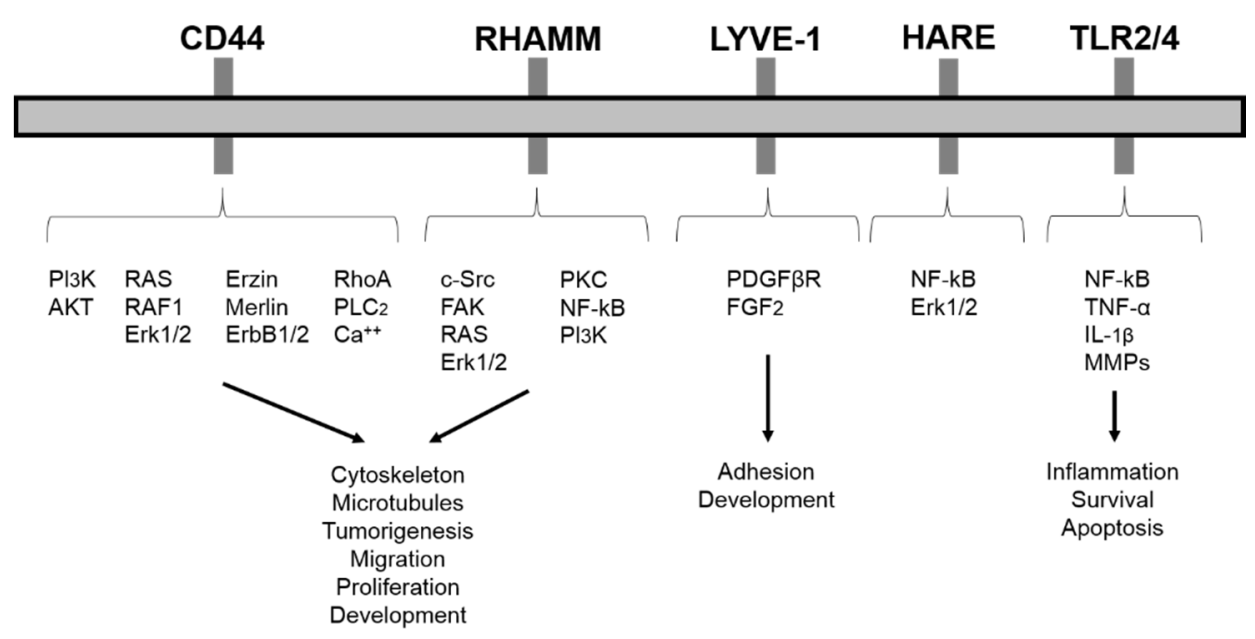

Figure 1. 5. Signalling cascade of the most important hyaluronan receptors and their role in cell and tissue functions [188].

\subsubsection{Size-dependent biological roles of HA}

HA presents different biological functions depending on its size, even $\mathrm{HA}$ of high (up to $400 \mathrm{kDa}$ ) and low $(0.2-400 \mathrm{kDa}$ ) molecular weights can regulate opposite signalling pathways and functions [189]. HA catabolism is regulated by the synthesis of new HA and degradation of the existing one. All the processes involved in the catabolism of HA are regulated by HYALs activity, and HA receptors play an important role in maintaining equilibrium between the low and high molecular weight forms, and as a consequence, catabolism regulates biological functions where HA is implied. Under certain pathologies synthesis of $\mathrm{HA}$, enzymatic activity of HYALs, endocytosis of HA and presence of ROS are increased, and can alter this equilibrium. In addition, the presence of abnormal sizes and concentration of HA in the blood stream or tissues may act as indicator of certain diseases.

Focusing on physical properties, HA of high molecular weight grants tissues lubrication, hydration and shock absorption, these functions are especially important in joints. Moreover, HA of high molecular weight shows the ability to bind molecules including cytoskeletal proteins and cell receptors. Thus, it acts as a structural molecule of the ECM, clusters receptors and gathers proteins on cell membranes, modulating several signalling pathways. Biggest chains have a protective effect on tissues against damages since it possesses antiinflammatory and immunosuppressive effects. These chains also cover receptors from cell surfaces impeding the activation. In wound healing, HA of high molecular weight favours the access for removing debris and bacteria and binding 
to fibrinogen. In cancer, it may suppress tumour progression because of its antiangiogenic effect and inhibition of migration. However, high molecular weight chains can impede regeneration of certain tissues, for example in cartilage it suppresses transcription factors such as collagen type II, implied in tissue repair.

On the other hand, HA fragments shows in many cases opposite effect to high molecular weight chains. The HA of low molecular weight displaces bigger HA chains from cell surfaces, destabilizing the native structures of the ECM and providing accessibility of stimuli to receptors on cell membrane. In addition, fragment sizes have specific responses in signalling pathways; receptor-bindings domains of HA have been observed in large chains (around $400 \mathrm{kDa}$ ) and in oligosaccharides containing only four saccharide units. In general, chains and oligosaccharides of low molecular weight up-regulates cellular functions including proliferation, adhesion, migration, angiogenesis and the immune response $[189,190]$. Thus, HA fragments have an important role in the inflammatory processes since they increase chemokine and cytokine production leading to the activation of macrophages and dendritic cells. In wound healing, HA of low molecular weight is responsible for the inflammatory stage and subsequent angiogenesis and fibroblast proliferation involved in the regenerative process. Moreover, HA fragments play important roles in the regeneration of other tissues, such as cartilage regeneration. However, HA oligosaccharides increase cancer progression because of their ability to cleave the receptor CD44, promoting cell proliferation (tumorogenesis) and migration (metastasis). In addition, HA fragments regulate other processes implied in cancer including stimulation of angiogenesis and suppression of apoptosis.

\subsubsection{Chemical modifications}

HA directly obtained from bacteria or found in animal tissues constitutes a GAG of high molecular weight. Despite the high molecular weight, therapeutic applications using $\mathrm{HA}$ are limited because of its poor mechanical properties, high solubility, fast degradation rate in physiological environment and poor adhesion with most cell types. Therefore, to expand the applications of HA for medical purposes, several chemical modifications have been studied in order to obtain insoluble derivatives and HA-based hydrogels. HA modifications are intended to improve its properties obtaining mechanically stable artificial ECMs, but retaining some biological properties of natural HA. The polyanionic nature and conformation in solution of HA determine the possibilities and conditions to perform chemical modifications. Therefore, the HA molecule shows two principal targets for chemical modifications: the carboxyl group from the GLCA unit and the unique primary hydroxyl from the GLCNAc unit. 
Chemical modifications of $\mathrm{HA}$ are aimed to obtain insoluble derivatives and crosslinked hydrogels from the direct crosslinking of native HA molecules or derivatives thereof. For these purposes, reactions are usually carried out in aqueous solutions. However, in these conditions, several reactions are $\mathrm{pH}$ dependent and some reagents must be protonated. Thus, reactions in aqueous solutions present a difficult control of the degree of substitution and a high batchto-batch variability, even extreme acidic or basic solutions are required inducing hydrolysis of HA chains [191]. For these reasons, several authors have investigated chemical modifications of $\mathrm{HA}$ in organic solvents, principally dimethylformamide (DMF) and dimethyl sulfoxide (DMSO). However, native HA associated with $\mathrm{Na}^{+}$cations is insoluble in organic solvents and it requires some previous modifications, including reduction of its high molecular weight and/or conversion into its acidic form or tetrabutylammonium (TBA) salt.

\subsubsection{Chemical modifications by the carboxyl group}

\section{$\checkmark$ Amidation employing carbodiimides}

The modification of $\mathrm{HA}$ with carbodiimides is commonly employed to functionalize $\mathrm{HA}$ chains since these reactions are relatively simple and it can be carry out into aqueous solutions. Carbodiimides modification is principally performed employing 1-ethyl-3-(3-dimethylaminopropyl)carbodiimide (EDC), which activates HA and, in the presence of an amine, an amino-acid ester is generated [192]. Therefore, EDC amidations consist in a two-step reaction; first EDC activates the carboxyl groups of HA chains forming an intermediate product (Oacyl-isourea) and, subsequently the amine or diamine produce a nucleophilic attack on the intermediate product leading to the amino-acid ester bond formation by an addition-elimination reaction. The reaction must be conducted at a $\mathrm{pH}$ range 3.5-6 because of the carboxyl group of HA is half hydrolysed and EDC protonation requires low $\mathrm{pH}$. However, the optimal $\mathrm{pH}$ for nucleophilic attack is alkaline one since amines require high $\mathrm{pH}$ to be deprotonated enough. Moreover, $\mathrm{O}$-acyl-isourea intermediate have a high reactivity and forms a by-product with water ( $\mathrm{N}$-acyl-urea). Thus, the requirement of different optimal $\mathrm{pH}$ values for each step and the formation of a secondary product constitute a difficult control on the reaction and low degrees of substitution (less than $40 \%$ ) [193].

In order to improve reactions by carbodiimides, researchers have developed some variations in the synthesis. Certain amines, such as 4-vinylaniline, show a less basic $\mathrm{pK}$ a value, and are more protonated at lower $\mathrm{pH}$ avoiding by-products formation in the nucleophilic attack [194]. Moreover, Biscarbodiimides can be employed as reagent itself to generate crosslinked hydrogels by stable $\mathrm{N}$-acylurea bonds. On the other hand, higher degrees of functionalization of HA chains, up to $55 \%$, may be reached replacing diamines by dihydrazides and 
sulphohydrazides because of their lower pKa values (2-3) [195]. In addition, the intermediate can be stabilized employing substances such as $\mathrm{N}$ hydroxysuccinimide (NHS) [196] or 1-hydroxybenzotriazole (HOBt) [197], accomplishing greater reactivity toward the amine. However, the highest degrees of substitution, between $60-80 \%$, in $\mathrm{HA}$ amidation using carbodiimides have been achieved by performing the reaction in organic solvents, either employing $\mathrm{HA}$ into its acidic form and TBA salts of HA [198].

Despite the disadvantages found employing carbodiimide in amidation reactions, they are widely used because of the possibilities to perform them in aqueous solutions and the lack of excessive cleavage of HA chains. Particularly, dihydrazides, such as adipic dihydrazide (ADH), are commonly employed to obtain functionalized groups for further modifications. This system has been investigated for coupling other polymers in HA chains, such as PEG [199], and drugs for cancer treatment including Taxol [200] and doxorubicin [201]. Moreover, HA functionalized with dihydrazides has been employed to develop crosslinked hydrogels [195].

\section{$\checkmark \quad$ Amidation using halogenated compounds}

Amidation of HA chains can be performed employing highly reactive compounds in order to activate carboxyl, including halogenated compounds. For these purposes, the most studied compound is the 2-chloro-1-methylpyridinium iodide (CMPI), whose reaction is performed in organic media such as DMF and DMSO to avoid CMPI hydrolysis [202]. The use of organic solvents requires to convert native HA in its TBA salt. CMPI acts as activator of carboxyl groups and produces a pyridinium intermediate by releasing $\mathrm{Cl}^{-}$, whose charge is neutralized by TBA ions. This amidation have been widely employed to obtain HA hydrogels by crosslinking the activated HA with diamines, which act to lead the nucleophilic attack. This crosslinking reaction improves the efficiency and it reaches degrees of substitution of $100 \%$, accomplishing rigid hydrogels with lower absorption of water. When no diamines are employed in the reaction, activated carboxylic groups of HA may react with its own hydroxyls giving an auto-crosslinked hydrogel by the formation of ester bonds between chains [203]. The advantage of these auto-crosslinked hydrogels is the lack of intermediate molecules, ensuring that hydrogels are formed only by native molecules. Thus, the degradation products of auto-crosslinked hydrogels by this method avoid undesirable products upon degradation [204].

In order to avoid the employment of organic solvents for obtaining HA derivatives by amidation, a novel halogenated compound have been studied, 2-chlorodimethoxy-1,3,5-triazine (CDMT) [205]. This compound activates the carboxylic groups of native HA chains by performing the reaction in mixtures of water and 
acetonitrile, and the released $\mathrm{Cl}^{-}$are neutralized by adding $\mathrm{N}$ methylmorpholinium (NMM). The HA intermediate has been reacted with amines, including 7-amino-4-methylcoumarin and propylamine, obtaining degrees of substitution between 3 and $20 \%$, respectively. This novel method has been employed to obtain functionalized HA chains but not crosslinked hydrogels. However, the authors suggest that hydrogels and higher degrees of substitution could be reached.

\section{$\checkmark$ Amidation with 1,1'-carbonyldiimidazole}

This method patented by Fidia consists in the activation of carboxyl groups from HA-TBA salt employing 1,1'-carbonyldiimidazole (CDI) in organic solvents [206]. The imidazole intermediate is more stable compared with previous described compounds, but its reaction time is longer. However, the reaction products of this amidation $\left(\mathrm{CO}_{2}\right.$ and midazole) show not toxicity. This amidation has been investigated to graft poly( $\mathrm{N}$-isopropylacrylamide) [207] and lipoic acid [208] into HA chains, obtaining thermoresponsive and more degradation-resistant hydrogels. Therefore, HA activated with 1,1'-carbonyldiimidazole may be interesting for applications such as drug and cell delivery.

\section{$\checkmark$ Esterification by alkylation}

Esterification of HA chains using alkyl halides allows the synthesis of amphiphilic HA derivatives from HA-TBA salts in organic chains. HA esterified with dodecyl and octadecyl chains employing alkyl bromides has been studied suggesting potential uses in viscosupplementation [209]. This reaction allows to modulate the degree of esterification of HA chains giving solutions with similar rheological properties to healthy synovial fluid because of the formation of transient hydrophobic interactions. Moreover, esterification by alkylation may be accomplished by activating carboxylic groups of HA with tosylate compounds, including PEG-tosylate to grafting PEG chains into HA [210]. However, these alkylation methods are not able to generate crosslinked HA hydrogels. For this reason, ditosylated compounds are employed to form crosslinks between HA chains, such as tetraethylene glycol ditosylate [210].

The firm Fidia (Italy) patented the esterification of HA using halides and alcohols (aliphatic, araliphatic, cyclo-aliphatic, aromatic, cyclic and heterocyclic series) under the trade name of HYAFF [211]. Fidia has developed a wide range of HA esterified derivatives with different properties by varying the degree of substitution and the employed alcohol/halide. These derivatives are water insoluble and maintain some biological properties of native HA with several improvements, such as longer degradation times and handling (they can be 
extruded and employed to fabricate scaffolds). HYAFF materials are employed for wound healing and are hopeful for other biomedical applications.

\section{$\checkmark \quad$ Esterification using epoxides}

Epoxides, particularly glycidyl methacrylate (GM), have been employed to obtain functionalized HA with glycidyl methacrylate groups (HAGM) with the aim of incorporating double bonds. GM reacts with carboxyl groups of HA by ring opening and with hydroxyl ones by transesterification, but the reaction by hydroxyls is reversible and carboxyl esterification is predominant [212]. The reaction is usually performed in aqueous media employing excess of GM and triethylamine as a catalyst, but the degree of substitution is low and shows a high batch-to-batch variability. Therefore, several researchers have performed the reaction in organic solvents using HA-TBA salt with GM, obtaining higher degrees of substitution and a more controllable reaction [213]. Incorporating double bonds into HA chains, made it available for further polymerization in order to obtain blended hydrogels with improved properties [214] and photocrosslinked hydrogels for in situ polymerization [213]. Moreover, HA functionalized with GM can be employed to graft other polymers for drug delivery [214] and attach HA on biomedical polymeric devices to avoid platelet adhesion [215].

\section{$\checkmark$ Hydrogels by UGl's and Passerini's condensations}

In the Passerini condensation a carbonyl and an isocyanide are condensed forming a protonated intermediate, which reacts with the carboxylic groups yielding, after rearrangement and translocations, an ester bond with the acid. In the UGl's reaction is included an amine which condenses fist with the carbonyl forming an imine, and the resulting compound consists in the carboxylic acid chain linked by amide bonds [216]. UGl's reactions to form HA hydrogels were carried out in acidic media employing formaldehyde, cyclohexylisocyamide and a diamine (lysine methyl ester or 1,5-diaminopentane), this mixture of reagents allows crosslink HA chains by the carboxylic groups [216,217]. This process accomplishes degrees of crosslinking from 3 to $10 \%$ showing different properties on swelling and mechanical properties, but further investigations are required in order to address a full knowledge of thee hydrogels. 


\subsubsection{Chemical modifications by the hydroxyl group}

\section{$\checkmark$ Ether formation using epoxides}

The reaction between epoxides and the HA hydroxyl groups is usually carried out in aqueous media under controlled $\mathrm{pH}$ giving an ether bond. This etherification consists in the ring opening of the epoxy group forming an electrophile intermediate which reacts with the hydrolysed anionic species into the HA chain. Epoxides can also react with both, carboxyl or hydroxyl groups of HA chains, depending on the $\mathrm{pH}$ of the reaction media [218]. Thus, at $\mathrm{pH}$ values up to 10 (pKa of hydroxyl groups), HA hydroxyl groups are deprotonated and the reaction forming ether bonds is predominant, whereas lower $\mathrm{pH}$ values generate, principally, esterification by the carboxyl groups. Despite the reactivity of both HA groups with epoxides, etherification is carried out commonly under alkaline conditions and heating forming the bond by the hydroxyls groups, principally, using bisepoxides to obtain crosslinked HA hydrogels. Laurent et al. patented the first HA hydrogel ever in 1964 employing 1,2,3,4-diepoxybutane leading to explore new systems in the subsequent years. Thus, several bisepoxides have been studied for HA hydrogels, including 1,4-butanediol diglycidyl ether (BDDE), Ethyleneglycol diglycidyl ether and polyglycerol, polyglycidylether, epichlorohydrin and 1,2,7,8-diepoxyoctane. BDDE chemistry is habitually employed for HA hydrogels with some therapeutic and cosmetic applications, including commercially available products such as Restylane ${ }^{\circledR}$ (Medicis Corp., AZ), Perlane ${ }^{\circledR}$ and Juvederm ${ }^{\mathrm{TM}}$ [219]. These products are employed as dermal fillers and have potential uses in other biomedical applications.

\section{$\checkmark$ Ether formation with divinyl sulfone}

Divinyl sulfone (DVS) is a chemical broadly employed to give HA crosslinked hydrogels $[220,221]$. The reaction is performed in alkaline conditions, typically with $\mathrm{pH}$ up 13, giving a bis-ethyl sulfone crosslink between the hydroxyls groups of HA chains (see Figure 1.6). The etherification by DVS consists in a Michael addition, and alkaline medium is needed to deprotonate hydroxyls groups of HA and provide a source of $\mathrm{OH}^{-}$. In alkaline media, DVS and deprotonated hydroxyls from HA react forming two ether linkages leading to the HA-DVS crosslinked hydrogel (Hylan). Unlike epoxides, this reaction occurs in few hours in alkaline media and at room temperature avoiding excessive cleavage of HA chains and undesirable products. On the other hand, despite the high toxicity of DVS reagent, HA-DVS hydrogels have shown not toxic degrading products [222]. The first HADVS hydrogel was patented by Balazs et al. [223] and since then, other authors have studied HA-DVS hydrogels. Hylans were the first HA-based hydrogels approved by the FDA for biomedical applications, concretely for dermal filling treatments. Balazs fabricated this first hydrogel in the early 1980 s and, currently, 
it is commercialized under the trade name of Hylaform ${ }^{\circledR}$ (Genzyme, NJ). In the recent years, other hylans have been developed for several biomedical applications, including ophthalmic viscosurgery (Hylashield® (Genzyme, NJ)), lubrication for knee joint (Synvisc $\circledast$ ) and anti-adhesion in surgery (Sepragel $\left.\AA^{\circledR}\right)$ $[219,224]$. In addition, DVS crosslinking have been employed with the aim to obtain $\mathrm{HA} /$ collagen hydrogels, resulting in non-cytotoxic effects and suggesting further use as dermal filler [225]. DVS is usually employed to crosslink HA molecules, whereas DVS and HA reaction can be stopped before completion obtaining unreacted vinyl groups, which are able to further conjugation of drugs and polymeric chains [226].

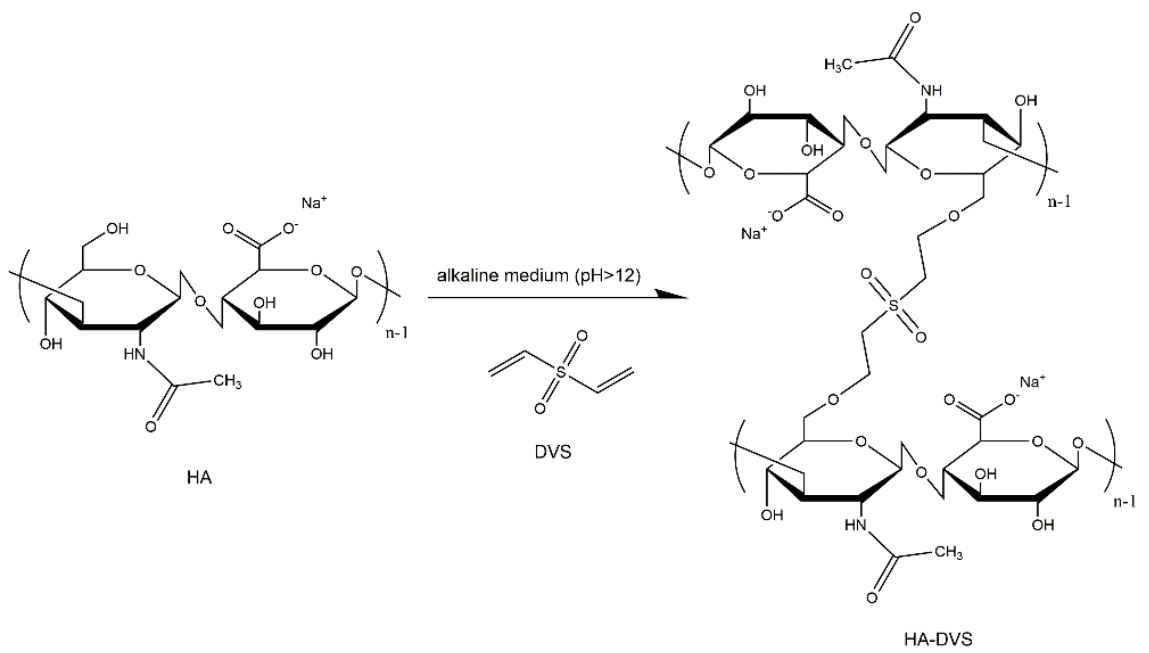

Figure 1. 6. Crosslinking reaction of divinyl sulfone with hyaluronan.

\section{$\checkmark$ Ether formation using ethylene sulphide}

A more recent method has been investigated in order to include ether bonds within the HA hydroxyls groups using ethylene sulphide as reagent [227]. The reaction is performed in alkaline media $(\mathrm{pH} 8.5-10)$ with further addition of dithiothreitol (DTT) to reduce any residual disulphide bond. The ethylene sulphide bond is opened by the action of protons from hydroxyls groups, giving a cationic molecule which performs an electrophilic attack into the deprotonated hydroxyls. As a result, the reaction generates HA chains with sulphide functional groups without crosslinking. The incorporation of sulphide groups reduces HA cleavage and shows a protective effect on chondrocytes under ROS, and this material may be also employed for the replacement of synovial fluid. 


\section{$\checkmark$ Hemiacetal formation employing glutaraldehyde}

Glutaraldehyde can be employed to form crosslinks between the hydroxyls groups of HA, leading hemiacetal bonds. The reaction may be performed in acetone-water mixtures or pure water under acidic conditions $(\mathrm{pH}=2)$ because of protons act as activators and catalysts [228,229]. In this reaction, protons from media bond to oxygen atoms from glutaraldehyde producing carbocations, which produces an electrophilic attack on HA hydroxyls and a hemiacetal crosslink is formed. However, the remaining glutaraldehyde is hard to remove and the hemiacetal crosslinks are unstable and can be hydrolysed in acidic media to its original products, generating cytotoxic and immunogenic responses [221]. In order to reduce glutaraldehyde residua it may be introduced in its vapour phase, avoiding unreacted reagents [230].

\section{$\checkmark$ Carbamate formation}

Cyanogen bromide has been employed to synthetize HA derivatives from its native sodium salt [231]. The reaction is performed in aqueous media at $\mathrm{pH} 10$ obtaining high degrees of substitution with an excess of reagents in only one hour. Thus, the cyanogen bromide reacts with the HA hydroxyls groups giving an activated $\mathrm{HA}$ cyanate ester, which is able to react with nucleophilic reagents such as amines. The reaction with amines also gives an HA carbamate product and an HA isourea by-product. HA derivatives with a broad range of tuneable properties can be obtained by varying the amount of reagents and the length of the amine chains [232].

\section{$\checkmark$ Ester formation}

Ester formation onto the hydroxyls groups of HA has been achieved employing several methods of synthesis: activated compounds such as acyl-chloride carboxylate compounds in organic solvents and anhydrides, such as octenyl succinic and methacrylic anhydrides, in aqueous media under alkaline conditions. The use of acyl-chloride carboxylate compounds was first reported with the aim to graft poly(lactic) oligomers onto the HA chains [233]. The compound to be grafted is previously activated by chloroacylation and the grafting is performed in DMSO by converting HA to its cetyltrimethylammonium bromide (CTA) salt.

Toemmeraas and Eenschooten patented a novel method to obtain HA derivatives forming ester bonds using aryl/alkyl succinic anhydrides (ASAs) in aqueous media at $\mathrm{pH} 9$ [234]. ASA-HA derivatives have been synthesized using octenyl and phenyl succinic anhydrides, reaching degrees of substitution up $40 \%$ [235]. 
These ASA-HA derivatives have demonstrated resistance against reactive oxygen species aiming its use for protecting organs and tissues [236].

Methacrylic anhydride (MA) has been widely used to graft unsaturated groups onto the HA chains by the formation of an ester bond (see Figure 1.7 A). Smeds et al. was the first to synthetize this hydrogel, since then, several authors have employed this system [237]. The reaction is usually carried out in alkaline aqueous media at pH 9-12 in a cold ice bath by adding an excess of MA, which reacts with HA hydroxyls by transesterification. The synthesis of methacrylated hyaluronan (MeHA) shows a difficult control because of the formation of methacrylic acid as by-product and the low degree of substitution reached. However, even low substituted MeHA may be further employed to perform photocrosslinked networks by free radical polymerization (see Figure 1. 7). The properties of MeHA networks may be tailored by varying native HA molecular weight, degree of substitution of the synthesized MeHA and its concentration in the prepolymer solution $[73,238]$. Thus, MeHA hydrogels have been obtained with volumetric swelling ratios from 8 to 48 , compressive moduli from 2 to 100 $\mathrm{kPa}$ and degradation times, in the presence of hyaluronidase, from a few hours to over one month. MeHA-based networks have been combined with collagen in order to obtain semi-interpenetrated networks (semi-IPNs) improving cell adhesion and mechanical properties [239]. The copolymerization of MeHA and poly(amino acids) leads to networks with longer degradation times than MeHAbased alone, and makes them able for drug release because of its behaviour in a pH-dependent manner [240]. Moreover, degradation times can be controlled by copolymerization of MeHA with hydrolytically degradable spacers including lactic acid and $\varepsilon$-caprolactone [241]. The MeHA product has been used to generate beads for chondrocyte encapsulation, suggesting future uses as injectable cell delivery vehicles [242]. Despite properties and in vitro cultures of MeHA networks have been researched by several authors, only few in vivo studies have been published. Thus, one in vivo study in rabbits with corneal laceration has demonstrated that MeHA hydrogels lack of excessive inflammation and permit the proliferation of stromal cells [243]. 
A

HA

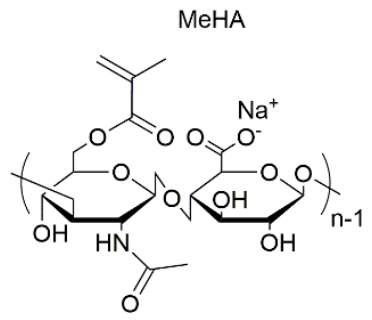

MA

B

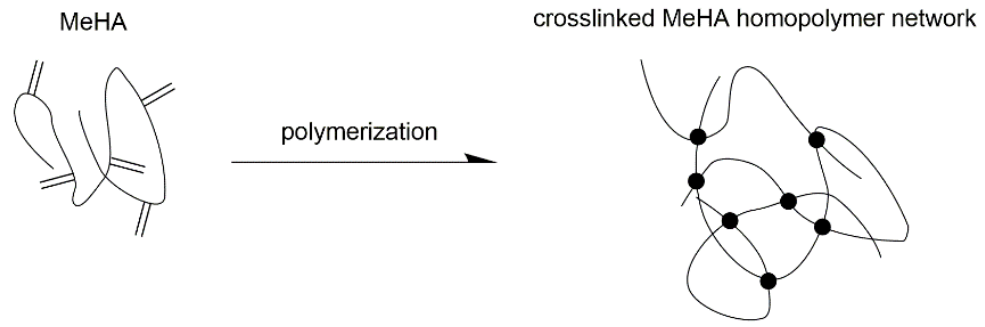

Figure 1. 7. Synthesis of methacrylated hyaluronan (MeHA) (A) and further polymerization (B).

\subsubsection{Other modifications}

\section{$\checkmark$ Modification by the $\mathrm{N}$-acetyl group}

The reactions by the $\mathrm{N}$-acetyl group from $\mathrm{N}$ - acetyl- D-glucosamine unit of HA require its previous deacetylation. Thus, the deacetylation of $\mathrm{N}$-acetyl group gives an amino group, which is able to react with electrophilic substances. The first method to carry out $\mathrm{N}$-deacetylation included an aggressive reaction such as degradation using hydrazine sulphate over long times and at high temperatures [206]. However, this method produces a reduction of molecular weight, and milder treatments have thereby reported by reducing hydrazinolosis times and addition of protecting functional groups [193,244] and employing the N-deacetylase enzyme [161]. Subsequently, deacetylated HA is usually reacted with acids to form amide bonds. The amidation of deacetylated HA has been employed to perform HA crosslinked hydrogels using glutaraldehyde [244], alginate obtaining copolymers [245], and UGl's reagents giving auto-crosslinked HA hydrogels [217]. 


\section{$\checkmark$ Oxidation}

Oxidation of hydroxyls and reducing end modifications produce reactive aldehydes, which are able for further modifications such as peptide attachment and crosslinking formation. The oxidation is usually performed using sodium periodate in $\mathrm{HA}$ aqueous solutions. The oxidized HA has been further employed to obtain in situ crosslinked hydrogels [246] and composites containing HA derivatives [247] other GAGs, such as heparin [248], and attached HA-peptides [249]. Moreover, HA derivatives have been oxidized to perform auto-crosslinked hydrogels, including thiolated HA oxidized using air, and HA-tyramine by adding horse-radish peroxidase (HRP) and peroxides [250,251]. However, this method can produce chain cleavage with reduction of molecular weight and undesirable immunogenic groups. On the other hand, reducing end modification is unexplored and limited to low molecular weights by the lack of large amounts of reducing ends in the biggest chains. This method has been employed to graft PLL onto HA chains by reductive amination between the reducing end of $\mathrm{HA}$ and the $\varepsilon$-amino groups of PLL employing sodium borate such as oxidizer agent [252].

\section{$\checkmark$ Hyaluronan complexation}

HA has a polyanionic nature and its ability to form hydrogen bonds making it useful to form complexes with cationic and other substances. Cations such as $\mathrm{Fe}^{+3}$ may chelate HA chains forming highly viscous semi-stable gels. Thus, The FDA approved in 2001 a novel HA hydrogel under the name of Intergel ${ }^{\circledR}$ (LifeCore, MN) obtained by chelation with ferric hydroxide. This hydrogel was employed to prevent adhesions during gynaecological surgeries [253]. However, the FDA is now urging customers to stop using this product because of the apparition of late post-operative pain by foreign body reaction. Moreover, HA has been employed to form complexes with other natural polysaccharides such as chitosan [254-257], which shows a polycationic structure with a large amount of hydroxyl groups leading to the formation of hydrogen bonds and electrostatic interactions between both polymers. Complexes composed of HA and chitosan have been manufactured by extrusion and layer-by-layer self-assembly techniques obtaining beds, films and membranes for drug delivery and tissue engineering purposes. On the other hand, HA may be complexed with substances, including DNA and RNA for gene delivery [258], BMP-2 for growth factor release in bone regeneration [259] and as a drug carrier [260]. 


\subsubsection{Medical applications of hyaluronan and its derivatives}

HA was first employed in burn and ulcer cares in 1960 and, at present, in a wide range of medical and cosmetic applications. As has been mentioned, HA is an important constituent of the ECM of several tissues granting the safety for therapeutic purposes because of its lack of excessive immunological response, compatibility and non-adherence with the surrounding host tissue. Moreover, HA interactions with some types of cells, such as chondrocytes and NPCs, regulating its functions. In addition, HA solutions show unique physicochemical properties because of its high molecular weight and polyanionic nature encouraging their use in some biomedical applications. From the viewpoint of the therapeutic use, the most important physicochemical properties of solutions containing high molecular weight HA include their ability to occupy large hydrated volumes and swelling. The viscoelastic properties of HA solutions conferring them applications such as filling spaces and protecting damaged tissues. In this direction, Balazs classified the therapeutic applications of HA based upon its viscoelastic properties: viscosurgery, viscosupplementation, viscoaugmentation, viscoprotection and viscoseparation. Nowadays, novel biomedical uses of HA are emerging, for example the preparation of drug and other carriers, and the fabrication of biomaterials for tissue engineering [261].

The first application of HA formulations on medicine were first performed in 1960 and 1970 by clinical trials in the ophthalmic surgery for corneal transplantation, providing protection to eye during the manipulation. This special surgery methodology is known as viscosurgery, and it is based on the principle that the vitreous humour of the eye is a viscoelastic fluid, whose major component is HA. Posterior findings provided highly purified HA leading to the first commercial product for viscosurgery, Healon ${ }^{\circledR}$ (Biotrics, MA), which are formulated as viscous solutions. Due to the promising results of this product, the use of HA was investigated for other ophthalmic surgeries, such as cataract ones, even it was employed in arthroscopic surgery to protect tissues and avoid their water loss. Since then, new Healon $\AA$ formulations and products were launched into the market with several HA molecular sizes. In order to obtain improvements in mechanical properties and long-term persistence, HA was mixed with other GAGs such as chondroitin sulphate, Viscoat (Alcon Labs, TX), and with hydroxypropylmethyl cellulose [262]. The problem of the current HA formulations is low residence time, and new HA based materials are also investigated, including crosslinked HA. The first crosslinking agents developed in preliminary studies of viscosurgery were DVS and formaldehyde, but the lack of structural coherence in these materials presents a problem [263]. Recently, new promising crosslinked hydrogels have been studied, including oxidized HA crosslinked with ADH [264]. Investigations are focused on optimizing crosslinked HA because of its better mechanical properties and long-term stability. 
Viscosupplementation employing HA is useful for orthopaedic surgery and rheumatology to replace damaged synovial fluid in joints affected by arthritis. Joints contain synovial fluid, which is composed of high molecular weight HA, between 2 and $7 \mathrm{MDa}$, giving lack of frictions and pain during movement. A disorder, such as arthritis, causes the degradation of the HA contained in the synovial fluid; its molecular weight decreases by the effect of neutrophils degenerating its viscoelastic properties and leading to chronic inflammation [265]. These disorders can be treated employing high molecular weight and concentrated $\mathrm{HA}$, which is also required to obtain similar viscoelastic properties of the healthy synovial fluid. Viscosupplementation employing HA was initiated in 1989 for the treatment of arthritis, and it is based on intra-articular injections of different HA formulations. Commonly, HA with high molecular weight, in the range of original one in the synovial fluid, was employed including different Healon $\circledast$ formulation (Pfizer, NY). However, formulations employing low molecular weight $\mathrm{HA}$ (about $1 \mathrm{MDa}$ ) are available, for example Hyalgan ${ }^{\circledR}$ (Fidia, Italy) and Artzal ${ }^{\circledR}$ (Seikagaku, Japan). Thus, low molecular weight HA have been demonstrated improvements on reducing pain without long-term effect [266]. In addition, bifunctional crosslinked $\mathrm{HA}$ has been formulated using epichlorohydrin obtaining hydrogels with high molecular weight (around $6 \mathrm{MDa}$ ), Synvisc ${ }^{\circledR}$ (Genzyme, MA). Injections of $\mathrm{HA}$ lead to some benefits in the patients; restoration of the viscoelastic properties of the synovial fluid, stimulation of chondrocyte proliferation and endogenous HA synthesis, moderation of inflammation due to the regulation of pro-inflammatory species, and analgesic effect [267].

The high hydrodynamic volume and water absorption of $\mathrm{HA}$ have been exploited for biomedical applications, which are referred as viscoaugmentation therapies. In viscoaugmentation, space filling of some tissues is required such as the filling of wrinkles and scars in cosmetics [268], Otolaryngology [269] and urinary incontinence [270]. Despite the appropriate use of HA for these purposes, its residence time is short and it is absorbed in a few days when is injected in solution. For this reason, researchers have investigated HA modifications to obtain crosslinked HA gels since 1960. The first crosslinked product surged in 1980 and it consisted in a DVS crosslinked HA hydrogel. Nowadays, they are a wide range of crosslinked HA gels in the market and approved by the FDA for dermal filling, crosslinked with DVS, Hylaform $\AA$ (Genezyme Biosurgery Inc., MA), and 1,4-butanediol diglycidyl ether, Restylane ${ }^{\circledR}$ (Medicis Corp., AZ). These commercial products have demonstrated good results and improvements in residence time respect to collagen materials in cosmetics [271]. Clinical trials employing HA gels for glottal fold insufficiency have been performed in recent years with promising results and longer residence times than collagen gels [272]. Despite the efficacy and safe of deep vocal fold injections of HA gels, other studies are necessary because of superficial applications have shown problems derived from its migration form the implant site [273]. HA solutions containing 
dextranomers and formulated as injections for urinary incontinence have been approved by the FDA; for clinical trials, Zuidex® (Q-med, NJ) and clinical uses, Deflux® (Salix Pharmaceuticals, NC) [274,275].

Other therapeutic applications of HA solutions and gels are focused on wound healing and tissue repair. The aim to employ HA in these fields is due to its ability to protect tissue surfaces from dryness or environmental agents, viscoprotection, and prevent adhesions of connective tissue and glial scar formation produced in surgical procedures or injuries, viscoseparation. Moreover, the potential uses of $\mathrm{HA}$ in wound healing and tissue repair are due to its anti-inflammatory properties, in high molecular weight formulations, and the angiogenic properties of its degrading products [167]. In recent years, HA has been present in a few clinical studies to determine its benefits in the treatment of ulcers, chronic wound and burns. For these purposes, authors have been employed several HA formulations, such as high molecular weight HA solutions [276], solutions containing bioactive molecules such as Hylactive ${ }^{\circledR}$ (Promedic, Spain) [125], and esterified HA such as Hyaff (Fidia, Italy) [277]. Despite the improvements found when HA is applied for wound healing, further studies are necessary to corroborate these results.

\subsubsection{Engineering of hyaluronan-based scaffolds}

At present, they are a few HA-based solutions and derivatives approved by the FDA and this fact has supposed the interest of several researchers on exploiting it for other biomedical applications. The current products of HA in the market are limited to HA solutions and a few derivatives and crosslinked hydrogels. However, in the last years researchers are interested on obtaining novel HA derivatives, hydrogels and materials with ECM-mimic structures (scaffolds) for a broad range of tissue engineering applications. Moreover, the use of HA to fabricate scaffolds shows many advantages because of its chemical structure, properties and presence in several tissues [278]. HA is a natural biodegradable material which is in many tissues, even it is the major component of connective tissue, playing important roles in cell behaviour, and it improves the healing because of its presence in all the steps of the wound healing process. Due to its chemical and conformational structure, HA can be easily modified to incorporate functional groups, active molecules and crosslinks. The HA molecule has low affinity for non-specific proteins, reducing scar formations and inflammation, but it shows domains for specific interactions with cell receptors involved in cell behaviour (CD44, RHAMM, etc.). The ability of HA to form hydrated domains, make it adequate for cell infiltration and nutrient transport. Thus, in this section are compiled the different methods to obtain HA-based materials, with special attention those where scaffolds of $\mathrm{HA}$ and derivatives thereof are obtained. 


\subsubsection{Phase separation}

Fabrication of scaffolds by phase separation consists on a thermodynamic demixing of a polymer solution into two phases: polymer-rich and polymer-poor phase. Demixing can be accomplished applying a non-solvent or chemicals, which form an insoluble phase, and cooling the solution below its freezing point. Then, the solvents are extracted by several methods including extraction, evaporation and sublimation obtaining a solidified structure of polymer from the polymer-rich phase. In the case of $\mathrm{HA}$, its intrinsic ability to absorb large amounts of water is exploited. Therefore, hydrogels and scaffolds of HA by phase separation are usually performed from $\mathrm{HA}$ aqueous solutions without employing other solvents. This method is known as freeze-extraction and it is based on freezing HA solutions and a subsequent sublimation. The structure is accomplished because of the growing ice nuclei through the polymer phase, and the further sublimation allows the formation of a solidified HA structure. Pore size and interconnectivity can be controlled varying ice nuclei formation applying different freezing temperatures and including salts, obtaining pore diameters below $200 \mu \mathrm{m}$ [278]. However, pore shapes are also influenced by HA concentration, molecular weight and composition (using native or HA derivative). This method allows obtaining 3D macrostructures with interconnected pores, but the disadvantage is the limited control of porosity. Manufacture of macroporous crosslinked HA scaffolds by freeze-drying have been widely explored, and several crosslinking agents have been employed, including glutaraldehyde, carbodiimide activation and DVS, resulting in differences in mechanical properties and swelling [279]. Despite the mentioned chemistries are the most employed to performance freeze-dried hydrogels, though, other ones have been studied including oxidized HA, crosslinking by epichlorohydrin, using derivatives such as methacrylated HA...

Several authors have focused their attention on obtaining composites of $\mathrm{HA}$ crosslinked with other polysaccharides and structural proteins, principally using carbodiimides. For these purposes, collagen has been widely crosslinked together HA by EDC-NHS chemistry, obtaining hydrogels with pores ranging from 100 to $200 \mu \mathrm{m}$ with improved mechanical properties and longer degradation times [280,281]. In order to obtain interpenetrating polymer networks (IPNs) of collagen/HA, collagen containing HAGM derivative has been crosslinked by carbodiimide reaction (semi-IPNs) and HAGM has been subsequently photopolymerized (full-IPNs) [282]. In addition, the collagen derivative, gelatine, has been linked to HA performing uniaxial porous structures around $150 \mu \mathrm{m}$ by controlling the freezing gradient, providing hydrogels excellent mechanical properties (elastic module until 2.9 MPa) [283]. Chitosan and agarose are both polysaccharides employed on obtaining composite scaffolds in combination with HA resulting in scaffolds with pore sizes between 100 and $200 \mu \mathrm{m}$, improved 
mechanical properties, longer degradation times and enhanced cell adhesion [284,285].

The principal limitation using HA-based scaffolds is the lack of binding sites for cell adhesion. For this reason, several authors have included proteins and sequences of peptides into HA chains by different methods. Grafting peptides is usually accomplished by activating HA scaffolds with $\mathrm{CDI}$, thereby coupling proteins such as LN [286] and sequences of peptides including IKVAV [85] and RGD [287]. However, other methodologies couple molecules and fabricate HA derivatives-scaffolds simultaneously applying different chemistries, this system has also been employed on including fibronectin (FN) fragments [288], poly-Dlysine (PDL) [289] and sequences of peptides such as RGD [290]. In addition to the incorporation of peptides and proteins, other molecules have been linked into HA scaffolds, including the antibody anti-Nogo-66 [291], growth factors [292] and DNA [293].

\subsubsection{Particle leaching and templating}

Unlike phase separation methods, the use of particle leaching and templates for scaffold fabrication allows the control over pore shape and porosity varying the porogen size and its relation respect to the HA solution, respectively. Particle leaching is based on mixing a polymer solution with porogen and subsequent removal of the solvent by different methods; whereas the use of templates implies a previous sintering of the porogen and the polymeric solution is introduced between the interstices of the resulting template. Currently, particle leaching is widely employed to fabricate porous scaffolds from a broad range of polymers, nevertheless this method is unexplored to develop structured HA hydrogels. HAbased scaffolds by particle leaching are usually prepared employing salts as porogen agents [294,295]. Despite salt leaching allows the control over pore sizes, the irregular shape of the particles leads to low porosities (around $70 \%$ ) and low connectivity. Similarly to the use of salt, ice particulate leaching has been employed as porogen obtaining similar results, because of their similar morphology [296]. The lack of high connectivity has aimed several authors to use templates, such as sintered poly (methyl methacrylate) (PMMA) beads by heating [297] or chemical solvents [298].

Regarding employed chemistries on HA-based scaffolds by particulate and template leaching, different crosslinking methods have been developed including EDC chemistry [296], incorporation of reactive acrylic groups [297] and employing benzyl ester derivatives such as HYAFF ${ }^{\circledR}$ [295]. Moreover, HA has been crosslinked with protein-derived molecules such as gelatine by EDC chemistry leading to composites with enhanced mechanical properties (elastic module around $5 \mathrm{MPa}$ ) [296]. In order to elicit bioactive scaffolds, several molecules have 
been incorporated within these HA scaffolds by different methods including polyplexes containing DNA-encoding for angiogenic growth factors [297] and covalently bonded peptides such as RGD [298].

Recent investigations are focused on obtaining porous structures by particle and template leaching of other polymers coated with HA hydrogels. Thus, HA-coated structures have been performed for different polymers including PCL [299], PLLA [300] and poly(ethyl acrylate) [301]. Moreover, chemical bonded HA forming coatings in porous scaffolds based on PLGA have been developed using pegylated molecules with amines and EDC chemistries [294].

\subsubsection{Electrospinning}

Electrospinning is a versatile system to perform polymeric thin fibres with diameters ranging from nano- to few micrometres. The principal differences of fibrous scaffolds respect porous conventional ones are their large surface areato-volume ratio, and high porosity with small pore sizes. In addition, the possibility of modifying several electrospinning parameters allow to fabricate a widely range of fibrous scaffolds morphologies including random and aligned fibres and more complex and precise structures, making this method able to perform biomaterials with topographical cues. The principle of the electrospinning process consists in the extrusion of a polymer solution through a small orifice at very high voltages, subsequently the evaporation of the solvent and the projection of the jet onto a metallic collector yield to a superposition of fibres forming a fibrous matrix. Size and morphology of the fibres can be modified by varying operation parameters such as polymer concentration, viscosity, polymer-solvents interaction, distance from the collector, applied voltage and external variables (temperature and humidity) [302].

Particularly, electrospinning of $\mathrm{HA}$ is difficult because the high viscosity and surface tension of HA aqueous solutions and its ability to retain large amounts of water produces a partial solubilisation of the HA fibres deposited on the collector due to the non-evaporated water. In order to reduce surface tension, electrospinning of $\mathrm{HA}$ is usually carried out from solutions containing organic solvents such as DMF [302]. On the other hand, HA aqueous solutions show high viscosity because the ability of $\mathrm{HA}$ to produce chain interactions and entanglement above the critical overlap concentration of polymer chains. However, the solution viscosity can be reduced using acids [303], alkalis [304] and dopant polymers such as poly(ethylene oxide) [305]. Moreover, water evaporation can be enhanced employing additional solvents such as ethanol [304], using electro-blowing [306], which incorporates a heater and a blower, and collecting the electrospun nanofibres in an ethanol bath [307]. 
Despite the difficulties on obtaining HA electrospun fibres, they can be performed with diameters in the range of $60-200 \mathrm{~nm}$, using the appropriate conditions. However, direct application of HA nanofibres into aqueous media, such as body tissues, shows a fast solubilisation of HA. In order to avoid a fast absorption of HA fibres, they must become water-resistant after or simultaneously the electrospinning process. The conventional methods include formation of HA fibres and posterior crosslinking using EDC chemistries [308] and not crosslinking methods have been developed to accomplish water-resistant fibres applying post-treatments including $\mathrm{HCl}$ vapour and acidic alcohol solutions [309]. Novel methods for obtaining crosslinked fibres are based on including a crosslinker agent into the electrospinable solution, for example this method has been employed in fibres based on HA-DVS [304] and 3,3'-dithiobis(propanoic dihydrazide)-modified HA (HA-DTPH) [305]. Other current methods consist in electrospinning of HA-derivatives such as MeHA, which is further polymerized [310].

The use of HA nanofibres for tissue engineering applications shows the disadvantage of a hard ingrowth of cells into the structure. For this reason, sieved $\mathrm{NaCl}$ has been uniformly dropped into the fibre during the electrospinning process, leading to nanofibrous scaffolds with porous structure [311]. Moreover, another major problem of HA fibres is its low ability to promote cell attachment, soft mechanical properties and high degradation rates. In order to enhance these properties, $\mathrm{HA}$ is usually electrospun simultaneously with structural proteins such as collagen [308] and gelatine [307] or co-electrospun with other polymers such as PLLA [304]. Moreover, when the requirement consists in improving cell attachment, peptide sequences such as RGD can be conjugated with HAderivatives yielding fibres containing peptide motifs [312].

\subsubsection{Rapid prototyping and patterning}

Rapid prototyping is used to obtain 3D scaffolds at micrometric scale by building successive layers of polymer under the control of a CAD software [313] or photopolymerization employing micro-mirrors that form reflective patterns [314]. The strategies employed on layer-to-layer fabrication are differentiated by the inkjet printing method, and include polymeric binders, fused deposition modelling and stereolithography applying photopolymerization to finish each layer. Rapid prototyping techniques have been developed using HA-derivatives solutions and each layer was solidified applying heat or photopolymerization methods. In order to improve water resistance, solidification by heating requires the combination of HA with other polymers [315], whiles stereolithography is carried out with methacrylated HA containing radical initiators [282]. However, HA derivatives are habitually printed in combination with other polymers including natural and 
synthetic ones. Moreover, printing of HA-based 3D structures can be performed by including cells and proteins [316].

On the other hand, patterning allows surface modifications on material surfaces at micro- and nanoscale. Therefore, they can be accomplished by common techniques including capillary force lithography, photolithography and electronbeam lithography. Due to the poor adhesion of cells on HA, capillary force lithography has been employed in the incorporation of HA pathways on substrates with the aim to obtain patterned surfaces with different ability for cell attachment [317]. Co-cultures onto these substrates resulted in the formation of aligned and separated cultures of each cell and favour cell-cell junction formation. Photolithography by spatially controlled light exposure of $\mathrm{HA}$ and collagen blends has been developed for obtaining photocrosslinked full-and-semi-IPNs [282].

\subsubsection{Micro- and nano-carriers}

In the last years, micro-and nano-carriers have been widely studied with the aim to obtain novel therapeutic systems with controlled drug release. According to the carrier nature, there are different types including solid lipids, dendrimers, polymers, nanogels, liposomes and aggregates of amphiphilic copolymers (micelles and vesicles). The possibility of incorporating different molecules into the carriers has encouraged researchers to apply these systems for several therapies such as gene therapy, drug release and protein delivery. Microcarriers have been principally synthesized for obtaining inhaled carriers, while nanocarriers are destined for performing injected systems. Currently, nano-carriers are in the focus of a broad range of investigations in order to obtain novel prodrugs with non-cytotoxic effects and longer circulation times.

The principal highlight of using HA as drug carrier is its biocompatibility, biodegradability, negative charge and their broad range of chemical and physical modifications. HA can be modified to incorporate drugs and obtain gels with tailored properties such as higher degradation times. The anionic nature of $\mathrm{HA}$ allows the modification of some common nano-carriers since positive charges form aggregates with serum proteins producing cytotoxicity and inflammation processes. HA-based carriers show great interest as tumor-targeting components because of a large number of HA-binding receptors are present into HA chains including the cell surface glycoprotein CD44, which is overexpressed in many tumoral cells. However, other HA-binding motifs are present such as the receptor for HA-mediated motility (RHAMM), the HA receptor for endocytosis of synovial epithelial cells in the liver (HARE), the lymphatic vessel endocytic receptor of endothelial cells (LYVE-1), and also intracellular HA-binding proteins including CDC37, RHAMM/IHABP, P-32, and IHABP4. 
Micro-carriers are usually fabricated by spray drying techniques, where the HA solution is extruded from a syringe at high flows and rapidly dried [318]. While nano-carriers are normally obtained by phase separation techniques, which consist in an emulsion of water and oil containing surfactants as stabilizers. On the other hand, drugs are usually conjugated with HA by chemical or physical linkages during carrier formation in order to obtain a more controlled and suitable release. Physical conjugation can be accomplished by non-covalent linkages such as ionic interactions, hydrophobic forces and hydrogen bonds, whereas chemical conjugation is performed by different reactions including amidation, esterification and etherification of HA carboxylic and hydroxyl groups and by amidation at its reducing end. The most habitual drugs conjugated with HA are those employed in chemotherapy in cancer, including Doxorubin [201], Taxols [319], hystones such as butyric acid [320], carbozanen containing boron atoms [321] and bisphosphonates [322]. Moreover, another compounds including nanocrystal semiconductors (Quantum Dots) [323] and gold nanoparticles [324] have been coupled into HA for imaging tumor cells and radiotherapy. Gene therapy is other promising area for cancer treatment. Thus, gene and oligonucleotides expressing anti-tumoral factors such as anti-vein endothelial growth factor (VEGF) have been included into HA nano-particles employing single-stranded DNA or RNA molecules $[325,326]$. Despite the development of HA carriers is principally focused on targeting tumor cells, some researchers have investigated alternative applications including conjugation with formyl peptide receptor like 1 (FPRL1) for inflammatory diseases [325], encapsulation of recombinant human insulin [318] and human growth hormone (hGH) [327] for diabetes treatment, and incorporation of erythropoietin (EPO) for renal insufficiency [328].

HA particles as carrier systems must avoid the release of drug in the physiological fluids before reaching the target. Therefore, depending on the type of stabilization, HA-carriers can be classified as chemical crosslinked, ionic complexes and hydrophophic associated complexes. Chemical crosslinked carriers are the most stable ones because of the incorporation of covalent bonds. In order to achieve covalent crosslinks, common chemistries have been employed including CDI-ADH chemistry, amide crosslinking using dihydrazides, ether formation using DVS, disulphide bonding from thiolated HA and using PEGaldehyde terminated groups [328]. The ionic complexation avoids the use of surfactants, organic solvents and chemicals that can affect the normal biological activity due to the entrapped molecules. Also, particles synthesized by ionic gelation require milder conditions, because of they are generated by electrostatic interactions between the HA carboxyl groups and components of cationic nature, such as chitosan [329]. An interesting method for obtaining HA carriers is by hydrophobic association of grafted segments coupled into HA chains, leading to micelle formation. Hydrophobic segments include polymers or copolymers such as PLGA, PEG, PCL, PLA and other substances, which are grafted using 
common chemistries previous micelle formation [330-332]. Amphiphilic micelles are further produced by interactions between the hydrophobic segments, requiring additional steps including sonication, exchanging and precipitation methods. Moreover, $\mathrm{HA}$ is widely employed for coating the surfaces of previously formed nanoparticles. In this case, HA acts as protector increasing the circulation, targeting tumor cells and reducing protein adhesion [333].

\subsubsection{Hyaluronan-based materials for CNS regeneration}

HA is an ECM component from the developing and mature CNS that has been employed as biomaterial for nervous system regeneration (PNS and CNS) because of its good integration with the host tissue (absence of immune response and glial scar formation), its similar mechanical properties with the neural tissue and the possibility to modify its properties (degradation and stiffness). HA-based biomaterials for neural regeneration have been commonly studied in $3 \mathrm{D}$ in vitro cultures [279], encapsulating cells [334], controlled release [319] and implantation in vivo after injuries in nerve [335], brain [286] and spinal cord [100]. Moreover, HA plays a fundamental role in brain development since neural precursor cells (NPCs) express this GAG in large amounts through the fetal stage, even these cells bind to this molecule modulating their differentiation $[336,337]$.

HA shows interesting properties for brain regeneration purposes, including biocompatibility, biodegradability and a high swelling degree. The HA products derived from its degradation process stimulate endothelial cell proliferation and migration promoting angiogenesis. The high swelling degree permits to accommodate and cover the whole lesion cavity, conferring mechanical properties similar to those of the brain tissue and allowing nutrients, ions and solutes transport. Besides, the high molecular weight HA has an antiinflammatory effect, allowing the control of microglia activation and avoiding foreign body reaction. HA-based scaffolds implanted in the brain have demonstrated to own angiogenic activity, inhibit formation of glial scar and secondary cavity, envelopes and cell aggregation [286]. The major limitation of $\mathrm{HA}$ is its poor cell adhesion; for this reason, it is usually employed with adhesion molecules or in combination with other polymers. HA-based scaffolds with immobilized LN have demonstrated improvements in the astrocyte response and the stimulation of neurite ingrowth, suggesting that HA-based scaffolds with these proteins promote neuroregeneration [286]. HA-based scaffolds with peptides such as IKVAV show similar effects than with LN immobilization, that is, a limited and localized inflammatory response, angiogenesis, neurite ingrowth and the complete integration of the scaffold with the host tissue [85]. The limitation to implant HA scaffolds is the invasive surgery required, but HA can be combined 
with other polymers to form gels in situ. For example, combining methylcellulose with HA thermoresponsive gels can be obtained, which, similarly to HA scaffolds, reduce the lesion cavity and attenuate microglia activation [338]. The combination of HA hydrogels with neuroprotective molecules, such as erythropoietin (EPO), promotes migration and proliferation of NSCs and NPCs from the subventricular zone (SVZ), reduces apoptosis and increases the amount of neurites, suggesting some neuroregeneration in the injured cortex [88]. The neuroregenerative effect of HA biomaterials can also be promoted by attaching receptor antibodies of inhibitory molecules for neural growth, such as NoGo [289].

Hyaluronic acid scaffolds, like collagen scaffolds, shows a good compatibility with spinal cord tissue but do not promote axon attachment and growth impeding regeneration. However, the implantation of HA gels in spinal cord lesions is able to reduce the lesion cavity, inflammation and glial scar in the surrounded tissue. The reduction of inflammation and gliosis is due to the own nature of HA: its anionic nature inhibits cell attachment and its CD44-binding motifs limit inflammatory effects [100]. However, HA can be functionalized or combined with other polymers to improve axon attachment and regeneration. The incorporation of PLL into HA gels can promote neurite attachment, while the incorporation of nogo66 receptor antibody promotes axon regrowth due to the agonist interaction with the inhibitory molecule nogo66 [339]. This system has yielded to the reduction of glial scar at injury sites promoting wound healing, angiogenesis and regeneration of both myelinated and unmyelinated axons. The combination of $\mathrm{HA}$ with methylcellulose (HAMC) produces gels in situ, which are employed for brain damages and SCl. The HAMC gel shows the advantage of forming porous gels in the lesion site covering entirely the lesion cavity and the possibility of incorporating factors or other neuroprotective compounds enhancing $\mathrm{SCl}$ regeneration. For instance, the incorporation of epidermal growth factor (EGF) and EPO in the HAMC gels has enhanced neuroprotection in the implant site and increased neuron density [340,341]. Thereby, HAMC hydrogels have been employed as NSCs and growth factor delivery in rat models of $\mathrm{SCl}$ showing improvements respect the implantation of cells alone. This system combining material as support, NSCs encapsulation and growth factor delivery increases survival of host oligodendrocytes and neurons, decreases lesion site and improves functional recovery. 


\subsection{Poly(methacrylates) and poly(acrylates)}

Poly(methacrylates) and poly(acrylates) are commonly referred to a wide family of polymers synthesized from the polymerization of methacrylate and acrylate monomers, which are esterified derivatives of methacrylic or acrylic acids, respectively (see Figure 1. 8). These monomers are composed of an ester group and a vinyl group. Vinyl groups become these monomers in Michael acceptors of radicals allowing the polymerization by addition reaction. They are commonly polymerized by radical polymerization using REDOX systems, thermal initiators or photoinitiators [342-344]. There is a broad range of initiators to perform this polymerization reaction and they are selected depending on the energy required for their decomposition and solubility in the prepolymer solution. Initiators require lower activation energies than methacrylate monomers to generate radicals and each molecule yields to the formation of two radicals by homolytic rupture. Subsequently, radicals react with the vinyl groups of the monomers giving an active carbon site at the monomeric unit. The reaction of polymerization continues by the reaction of the active site with new vinyl of other monomers leading to the propagation of the reaction. The termination reaction in the polymerization is produced by the combination of two active sites or the transference of hydrogens within the active site. These termination processes yield the formation of inactive macromolecules (polymeric chains).

In order to obtain crosslinked polymer networks, methacrylate monomers are usually polymerized together with crosslinking agents such as glycol-based molecules containing almost two acrylate groups [343]. The incorporation of crosslinks within the network allow the control over properties and they produce materials with improving stability. In addition, monomers or polymers lacking of double bonds may be methacrylated by several chemical pathways such as the case of MeHA [241] described above. The incorporation of double bonds allows the polymerization and copolymerization of these molecules via radical, leading to a wide range of new polymers and copolymers. 


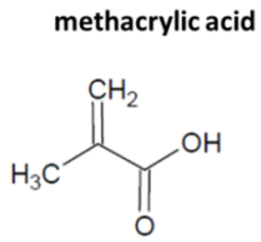

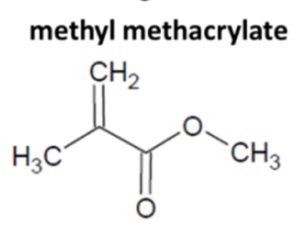

ethyl methacrylate<smiles>C=C(C)C(=O)OCC</smiles>

acrylic acid<smiles>C=CC(=O)O</smiles>

methyl acrylate<smiles>C=CC(=O)OC</smiles>

ethyl acrylate<smiles>C=CC(=O)OCC</smiles>

Figure 1. 8. Methacrylic and acrylic acids and some derivatives thereof.

The most common poly(methacrylates) and poly(acrylates) employed in tissue engineering include two differentiated groups depending on the end termination of their ester chain: poly(methacrylates)/poly(acrylates) and their hydroxy forms. The ester chain in poly(methacrylates) and poly(acrylates) is composed of simple alkane groups, while their hydroxy forms contain a hydroxyl group at the termination of the ester chain. The principal differences between both groups are their hydrophilicity and solubility in water: poly(methacrylates) and poly(ethylacrylates) are relatively hydrophobic and soluble in organic solvents, while the hydroxyl forms are hydrophilic polymers with some degree of solubility in water. On the other hand, networks based on these polymers show differences related to their swelling properties in aqueous media; thus, poly (methacrylate) and poly(acrylates) polymer networks lack of significant swelling in aqueous media and the polymer networks based on the hydroxy forms can absorb relative large amounts of water.

The properties of each polymer are quite different and depend on monomeric or co-monomeric compositions, polymerization conditions and the addition of crosslinking agents [345]. The methacrylate polymers show better chemical, mechanical and thermal properties than acrylate ones, and the hydroxy-polymer forms show in general a reduction of these properties. Normally, the larger the lateral ester chains, the lower the thermal properties (temperatures of vitreous transition and fusion), chemical resistance and mechanical properties (reduction of rigidity). This variation in properties is consistent with interactions and packing of functional groups in the repeat units along the polymeric chain. Additionally, 
hydroxy-polymer forms show higher temperatures of vitreous transition than the poly(methacrylates) and poly(acrylates) ones.

Particularly, poly(ethyl acrylate) (PEA) block polymer possesses temperatures of vitreous transition around $-10 \stackrel{\circ}{\circ}$ (this temperature noticeably varies for PEA networks and depends on the conditions employed in the polymerization reaction) $[346,347]$. Unlike PMMA, which has a temperature of vitreous transition around $110^{\circ} \mathrm{C}$, PEA networks are completely amorphous at physiological temperatures, leading to flexible polymers and networks. Therefore, PMMA shows Young's modulus around 2-3 GPa, while PEA one is around $2 \mathrm{MPa}$.

\subsubsection{Poly(methacrylates) and poly(acrylates) in tissue engineering}

Poly(methacrylates) were the first synthetic polymers employed in tissue engineering as contact lenses. Since then, poly(methacrylates), poly(ethyl acrylates) and the hydroxy forms thereof have been widely employed in several tissue engineering applications including clinical treatments. Some of these most studied in tissue engineering include PMMA, poly(ethyl methacrylate) (PEMA), poly (methyl acrylate) (PMA) and PEA. In addition, the hydrogel nature of the polyhydroxy forms has encouraged their use in many tissue engineering applications, of which the most employed include PHEMA and PHPMA.

All these polymers are bioestable materials and PMMA is the one with higher mechanical, thermal and chemical resistance properties. Therefore, this polymer is the most widely employed in therapeutic applications, principally, for permanent bone and dental cements. Moreover, PMMA was employed in the fabrication of rigid contact lenses because of its good optical properties [348], and this material is currently used as replacement of the native cornea. Other emergent application of this polymer includes its use as dermal filler together with hydrogels. However, high rigidity and hydrophobicity of PMMA encouraged its combination with other acrylate or methacrylate polymers, such as PHEMA giving a more flexible and hydrophilic material, and PEA in order to obtain more flexible and impact resistant dentures. In addition, the different methods to polymerize methacrylates have leaded a broad range of application forms in clinic: injections of microparticles, previous moulded prosthesis and polymerization in situ [349,350].

Contrarily to PMMA, PEA constitutes a less explored material for tissue engineering applications. However, PEA shows interesting properties make it attractive for some applications, especially in those where flexibility is required such as soft tissue engineering. Moreover, PEA has been polymerized by different techniques in order to obtain scaffolds with different shapes: aligned and interwoven channels and porous scaffolds $[49,301,344]$. Porosity leads to PEAbased scaffolds lower mechanical properties reducing its Young's modulus from 
$2 \mathrm{MPa}$, in block polymers, to $0.1-0.2 \mathrm{MPa}$, in porous ones. Habitually, polymerization of PEA includes a crosslinking agent leading to networks with improved bioestability. The principal potential fields of PEA include soft tissue engineering such as neural one.

\subsubsection{Poly(methacrylates) and poly(acrylates) in central nervous system regeneration}

In general, substrates based on poly(methacrylates) and poly(acrylates) present good adhesion of cells and may be obtained in multiple fashion [31]. The hydroxyl-terminated polymers may be tailored with similar properties to CNS tissues, but they show lower cell adhesion than the non-hydroxyl-terminated ones. Several in vitro studies employing neural cells have been developed using methacrylate polymers and derivatives thereof, including patterned substrates [351], aligned fibres [27], channel conduits [49] and porous scaffolds [344].

Micro- and nano-patterns of PMMA and its derivatives have resulted in good neural adhesion and the ability to orient neurite outgrowth. Moreover, sprouting and length of the processes can be increased by selecting the appropriate dimensions of the patterns $[31,36]$. Conduits containing sheets of aligned fibres based on PMMA and acrylonitrile copolymers have been developed for $\mathrm{SCl}$ models [48]. Cultures of DRG neurons in these constructs have resulted in the aligned outgrowth of axons. In addition, these conduits have been combined with an electrode array recording evoked neural activity [27]. Neurite growth has been oriented by combining molecular cues, such as growth factors and proteins, with polymeric substrates. In this sense PMMA sheets have been combined with LN pathways with different width observing improved neurite outgrowth and orientation in those with dimension between 30-50 $\mu \mathrm{m}$ [351]. On the other hand, porous scaffolds composed of PHEMA containing NT-3 and NGF gradients have resulted in the regrowth of axons to the higher growth factor gradients [352]. In vitro studies have shown that PEA is a good biomaterial as substrate for different glial and neural cells, including Schwann cells and NSCs [21,353] and endothelial cells [354]. In the case of NSCs cultures, the differentiation of these cells to neural lineages was improved employing copolymers of PEA and PHEA with higher hydrophilicity than PEA alone [21].

The PHEMA and PHPMA are the most common methacrylate substrates studied for brain regeneration, because of its ability to form hydrogels, yielding substrates with similar properties to the natural tissue. Both polymers show a good biocompatibility and integration with the host tissue adhering firmly to the surrounding brain tissue [355]. The implantation of crosslinked PHEMA and PHPMA porous scaffolds leads to a dense glial scar and chondroitin sulphate proteoglycans (CSPGs) surrounding the material, whereas this glial layer is 
discontinuous promoting the infiltration of astrocytes, tissue compounds (fibroblasts, collagen and blood vessels) and a small amount of neurites. Interestingly, the inhibitory substances and cells of axon growth (CSPGs and inhibitory cells including oligodendrocytes and microglia) did not invade the hydrogels. PHPMA scaffolds show improvements for brain regeneration with respect to PHEMA ones due to the infiltration of larger amounts of neurites and connective tissue into the hydrogels. However, these hydrogels present a low cell adhesion, which can be enhanced by the incorporation of adhesive peptides. Thus, the immobilization of IKVAV peptide on PHPMA leaded to the structural continuity across cavity, resulting in a more adequate substrate for axonal ingrowth [356]. The immobilization of RGD peptides on PHPMA porous scaffolds enhances cellular attachment and infiltration; even they promote neurite regrowth and ameliorate astrocyte reactivity [357]. These systems have been combined with engineered fibroblasts to express neurotrophic factors obtaining larger amounts of regrowing axons, less astrocyte proliferation and similar inflammatory response when both neurotrophic factors are secreted together [358]. In addition to these common polymer employed in brain regeneration, PEA scaffolds with aligned and interwoven channels have been implanted near the SVZ of rat brains. This study suggests that PEA channels are able for the ingrowth of neural, glial and endothelial cells.

Similarly to brain regeneration, PHEMA-and PHMA-based hydrogels have been compared in studies of spinal cord repair [357]. Both hydrogels show a good integration with the host tissue, presenting a minimal glial scar and cystic formation and the infiltration of connective tissue. The hydrogels have been found to promote the infiltration of blood vessel and axons into the pores, even axons have grown into the central part of the hydrogels after eight weeks. Moreover, SCs infiltrate into the hydrogels and along the axons, suggesting the possibility of obtaining meylinated axons. These hydrogels can be modified with peptides and neurotrophic factors to improve axon regeneration and generate neuroprotection. Thus, the incorporation of the cell adhesive site of FN, RGD (NeuroGel ${ }^{\mathrm{TM}}$ ), into PHMA hydrogels with a porous structure obtained by freezedrying has been found to promote the axon regeneration in spinal cord repair [357]. These systems provoke an early inflammation at few days postimplantation, which decreases after a few days. One week after implantation, the proliferation of capillary sprouts, glial scar surrounding the implant and few amounts of fibroblasts, fibrocytes and macrophages can be observed. Over the next weeks, the implants showed ingrowth of capillaries, infiltration of connective tissue, glial processes and axons. Moreover, the implants presented neither cystic formation nor secondary axon degeneration, and the axonal pathways were altered into the rostral stump, showing signs of morphological changes and regeneration indicating growth cone formation. Similar studies on PHPMA hydrogels with immobilized RGD and neurotrophic factors (BDNF and CNTF) 
have resulted in a major number of axons growing in the hydrogels compared with unmodified ones [358]. Conduits of PHEMA-co-methyl methacrylate with different internal matrices (collagen, fibrin, matrigel and methylcellulose) and growth factors (FGF and NT-3) have been compared with multichannel conduits and have demonstrated improvements in the regeneration of selective tracts in spinal cord injuries [110]. This study shows that all the internal matrices increase the axons density within the channels except the collagen matrix without growth factors and the multichannel conduits without fillings. The internal matrix did not influence the regeneration of sensorial axons and axon growth factors decrease their ability to regeneration. The different matrices and growth factors affect selectively to different tracts: fibrin promotes the greatest axonal regeneration of reticular neurons, methylcellulose of vestibular and red nucleus neurons, FGF increases the axonal regeneration of vestibular neurons, and NT-3 decreases the total amount of axons regenerating from brainstem neurons. In addition, fibrin and multichannels accomplish improvements in the locomotor function. With the aim to improve axon regeneration in the injured spinal cord, PHMA- and PHEMAbased hydrogels have been combined with MSCs, which show the ability to deliver neurotrophic factors [359]. In a preliminary study, the survival of MSCs within PHMA and PHEMA implants has been compared, being it higher in the PHMA hydrogels. Besides, this work also demonstrated that most of the cells locate in the pores but a few cells migrate to the border zones of the spinal cord, pointing out that the use of these hydrogels can be helpful as carriers of stem cells and their delivery in the lesion site of the spinal cord. 
Table 1. 1. Summary of the characteristics of the CNS tissue and comparison of the main physicochemical and biological properties of HA and PEA.

\section{CNS}

HA

Tissue composed of different cell types and structures including the neuronal network, the microvasculature and support cells (glia). Unlike other tissues, its ECM lacks of large amounts of collagen and it is mainly based on GAGs combined with proteins and other molecules. The cell structures and ECM components provide a highly porous and hydrated tissue with soft mechanical properties (Young's modulus between 0,1 and $10 \mathrm{kPa}$, depending on age and specific CNS tissue)

It is characterized by a limited regeneration ability because of the lack of neurons replacement and the inhibitory environment generated after a damage, yielding to a secondary cell death and permanent loss of functions tissues angiogenesis
Natural GAG occurring in the ECM of several Synthetic polymer derived

Biodegradable
Hydrophilic and forms
hydrogels when is
crosslinked

High molecular weight forms are characterized by a low adhesion of cells and proteins, protection of cells against chemicals, suppression of the immune response and

\section{Low molecular weight forms favour angiogenesis, proliferation and migration of cells, and induce inflammatory effects}

Polyelectrolyte containing negative charges and hydrogen bonds between adjacent disaccharides

\section{Biostable}

Hydrophobic and the polymer networks thereof are characterized by a low water uptake

Fibrillogenesis and cell attachment is stimulated onto its surface
In the solid state, it is a vitreous material at physiological temperature and lacks of thermal transitions in a wide range of temperatures

Soft mechanical properties of swollen crosslinked hydrogels (Young's modulus ranging from few $\mathrm{Pa}$ to a few hundreds $\mathrm{kPa}$ )
Uncharged polymer owing low interactions between groups

Amorphous with a elastomeric behaviour at physiological temperature ( $\mathrm{Tg}$ around $-10^{\circ} \mathrm{C}$ )

\section{1 and $2 \mathrm{MPa}$}

Young's modulus between 


\subsection{Hypotheses and objectives}

\subsubsection{Hypotheses}

The aim of this work is the development of structures, mainly based on hyaluronan derivatives, having long residence time in the physiological environment, resistance to the hyaluronidase activity, tuneable mechanical properties and swelling degree, structural stability during the regenerative process and good compatibility with cells belonging to the brain tissue.

The specific hypotheses are the following:

- the crosslinking of HA with DVS allows the development of insoluble scaffolds containing a stable structure

- the degree of crosslinking might modulate the mechanical properties, swelling degree and the initial colonization of glial, neural and endothelial cells

- the post-treatments on the crosslinked HA with DVS may inhibit the action of hyaluronidase

- the methacrylation of HA using MA may provide vinyl groups in the HA molecule, and hence this HA derivative, MeHA, may be copolymerized with other monomers

- the copolymerization of MeHA with other monomers permits the development of copolymers combining the properties of HA and other polymers

- the copolymerization of MeHA and EA yields semi-biodegradable copolymers with tuneable properties and structural stability during the regenerative process

- the initial cell colonization of glial, neural and endothelial cells may be improved in these copolymers due to the PEA fraction

\subsubsection{Objectives}

The main goal in this work is the development of HA-based materials with longer resistance against enzymatic degradation, longer residence time in the physiological environment and controllable properties (mechanical and biological). In order to accomplish this main objective, two strategies have been explored: 1) crosslinking of HA with divinyl sulfone (DVS) followed by posttreatments to improve the resistance against to hyaluronidase activity, and 2) copolymerization of a HA derivative, methacrylated $\mathrm{HA}$ (MeHA), with a biostable 
polymer, poly(ethyl acrylate) (PEA), to yield novel semi-degradable materials, poly (MeHA-co-PEA) copolymer networks.

From each strategy, several specific objectives were derived:

HA crosslinked with DVS

- characterization of the crosslinking of HA with DVS taking into account the polyelectrolyte nature of HA polymer networks. The characterization includes the development of a semi empirical method to determine the degree of crosslinking and determine the efficacy of the crosslinking reaction

- development of a repetitive process to obtain HA crosslinked scaffolds with tailored properties

- chemical, physical and mechanical characterization of the HA crosslinked scaffolds

- determination of the influence of the post-processes employed in water removal over the degradation of HA polymer networks

- biological evaluation of the crosslinked HA scaffolds with cell populations mimicking those found in the brain environment (endothelial, neural and glial cells). Additionally, the effect of the degree of crosslinking on the initial colonization will be determined

Poly(MeHA-co-EA) copolymer networks

- modification of HA to provide double bonds with the aim to perform further polymerization reactions. Determination of the substitution degree

- development of novel semi-degradable copolymer networks, poly(MeHAco-EA), with different compositions

- determination of the real components using several techniques and study of the phases distribution in the different copolymer networks

- physical, chemical and mechanical characterization of the copolymer networks determining the relation between composition and properties

- determination of the hyaluronidase effect over the copolymer networks studying the integrity of the biostable component and the by-products produced during the degradation

- biological evaluation of the copolymer networks in terms of its cytotoxic and inflammatory effects on endothelial, glial and neural cells 

CHAPTER II 



\subsection{Materials and cell lines}

\subsubsection{Polymers and monomers}

$\checkmark$ Hyaluronan sodium salt from Streptococcus equi. (HA, $1.63 \times 10^{6}$ Dalton) was supplied by Fluka.

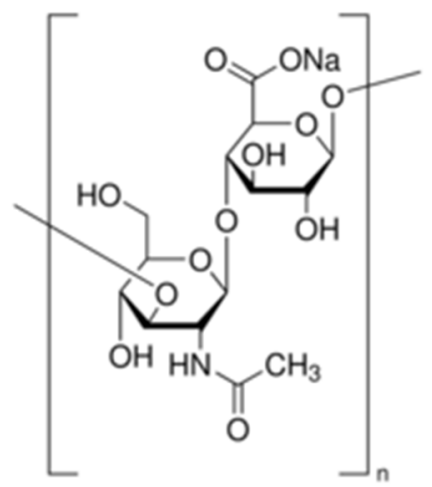

$\checkmark$ Ethyl acrylate (EA, $99 \%)$ was purchased from Aldrich.<smiles>C=CC(=O)OCC</smiles>

EA was employed in the copolymerization with modified HA.

$\checkmark$ Methacrylic anhydride (MA, $94 \%$ ) was purchased from Aldrich.<smiles>C=C(C)C(=O)OC(=O)C(=C)C</smiles>

MA was employed in the HA methacrylation process. 
$\checkmark$ Poly(ethyl methacrylate) (PEMA, $50 \mathrm{kDa}$ ), Elvacite® 2043, was supplied by Lucite International, Inc.<smiles>CCOC(=O)C(C)(CC(C)(C)C)C(C)(C)C</smiles>

PEMA spherical beads with particles sizes from 140 to $220 \mu \mathrm{m}$ were employed as porogen.

\subsubsection{Crosslinking agents}

$\checkmark$ Divinyl sulfone (DVS, at $97 \%$ ) was provided by Aldrich.<smiles>C=CS(=O)(=O)C=C</smiles>

DVS was employed as crosslinking agent of HA chains.

$\checkmark$ N,N'-methylenebisacrylamide (MBAA, at $99.5 \%$ ) was supplied by Aldrich.<smiles>C=CC(=O)NCNC(=O)C=C</smiles>

MBAA was employed as crosslinking agent in the free radical polymerization reaction.

\subsubsection{Initiator}

$\checkmark$ Potassium persulfate $\left(\mathrm{K}_{2} \mathrm{~S}_{2} \mathrm{O}_{8}, 99 \%\right)$ was supplied by Aldrich.<smiles>O=S(=O)(O)OOS(=O)(=O)O</smiles>

$\mathrm{K}_{2} \mathrm{~S}_{2} \mathrm{O}_{8}$ was employed as thermal initiator in the free radical polymerization reaction. 


\subsubsection{Other reagents}

$\checkmark$ Sodium hydroxide $(\mathrm{NaOH}, 98 \%)$ was provided by Scharlab. $\mathrm{NaOH}$ was employed as catalyser in the crosslinking reaction of HA.

$\checkmark$ Acetone and ethanol (synthesis grade) were provided by Scharlab. Acetone and ethanol were used in the extraction process.

$\checkmark$ N,N-dimethylformamide (DMF, $99 \%$ ) was purchased from Scharlab. DMF was employed as solvent in the chemical modification of HA.

$\checkmark$ Phosphate Buffered Saline (0.15 M PBS). 0.15 M PBS was employed as solvent in the chemical modification of $\mathrm{HA}$.

\subsubsection{Cell lines and culture media}

$\checkmark$ Human Cerebral Microsvacular Endothelial Cell line (hCMEC/D3)

Human Cerebral Microvascular Endothelial Cell line (hCMEC/D3) is immortalized after transfection with Simian Vacuolating Virus $40 \mathrm{~T}$ antigen (SV40 TAg) and a human telomerase. hCMEC/D3 cell line were provided by the group of PierreOlivier Couraud (Department of Cell Biology, Institute Cochin, Paris, France). hCMEC/D3 cell line was expanded on fibronectin-coated culture flasks in Endothelial Cell Basal Medium (ECBM, see below). hCMEC/D3 cell line were sub-cultured twice a week at a dilution 1:3 from confluence and employed for different assays between passages 25-30 and using ECBM as culture medium.

\section{Pig brain endothelial cells (pBECs)}

Pig Brain Endothelial Cells (pBECs) were extracted from pig brains and provided by Dr. Freese (Institut für Pathologie, Universitätsmedizin Mainz, Germany). pBECs primary cells were expanded on FN-coated culture flasks in ECBM as culture medium for 2 weeks. pBECs were used after the first passage. ECBM was replaced twice a week. 
$\checkmark$ Human glioblastoma-astrocytoma cell line (U373)

Human glioblastoma-astrocytoma cell line (U373; ATCC code HTB-17) was purchased from the American Type Culture Collection (ATCC). U373 cell line was expanded on culture flasks with minimum essential media (MEM, supplemented with $15 \%$ of Foetal Calf Serum (FCS), 10000 units $/ \mathrm{mL}$ penicillin, $10000 \mu \mathrm{g} / \mathrm{mL}$ streptomycin, $1 \%$ Glutamax and $1 \%$ sodium pyruvate) supplied by Gibco. U373 cell line was sub-cultured at a dilution 1:3 from confluence, with the same medium, twice a week. The in vitro experiments were performed using U373 cell line at passages 20-25 using ECBM as cell culture medium.

$\checkmark$ Neuroblastoma cell line (SH-SY5Y)

Human neuroblastoma cell line (SH-SY5Y; ATCC code HTB-11), a thrice cloned (SK-N-SH $\rightarrow$ SH-SY $\rightarrow$ SH-SY5 $\rightarrow$ SH-SY5Y) sub-line of the SK-N-SH (neuroblastoma cell line), was supplied by the American Type Culture Collection (ATCC, Manassas, VA). SH-SY5Y cell line was expanded using Dulbecco's Modified Eagle Medium/Nutrient Mixture F-12 (DMEM/F12, supplemented with $10000 \mathrm{units} / \mathrm{mL}$ penicillin and $10000 \mu \mathrm{g} / \mathrm{mL}$ streptomycin) and supplied by Gibco. SH-SY5Y cell line was sub-cultured twice a week and employed between passages 15-20, using ECBM cell culture medium.

\section{$\checkmark$ ECBM}

hCMEC/D3, pBECs, U373 and SH-SY5Y cell populations were cultured in endothelial cell basal medium (ECBM, containing $28 \mathrm{mM}$ hydroxyethyl piperazine ethanesulfonic acid (HEPES)) and supplied by Promo Cell. ECBM was supplemented with $15 \%$ of FCS, 10000 units $/ \mathrm{mL}$ penicillin and $10000 \mu \mathrm{g} / \mathrm{mL}$ streptomycin purchased from Gibco, $10 \mu \mathrm{g} / \mathrm{mL}$ sodium heparin and $2.5 \mathrm{ng} / \mathrm{mL}$ basic fibroblast growth factor (bFGF) and supplied from Aldrich. 


\subsection{Methods}

\subsubsection{Crosslinking of hyaluronan polymer networks}

Divinyl sulfone-crosslinked hyaluronan polymeric networks (solvent-cast HA-DVS polymer networks) with different HA:DVS molar ratio, referred as molar ratio of HA monomeric unit to DVS, were obtained (see Table 2. 1). First of all, a solution of $50 \mathrm{mg} / \mathrm{mL}$ of hyaluronan sodium salt in $0.2 \mathrm{M}$ sodium hydroxide was prepared shaking for 24 hours and subsequently stirring at $200 \mathrm{rpm}$ for 1 hour. Afterwards, the corresponding amount of DVS was added dropwise into $10 \mathrm{~mL}$ of the HA solution and stirred at $300 \mathrm{rpm}$ for 1 minute. Finally, the resulting solution was poured on a petri dish $(8.5 \mathrm{~cm}$ of diameter) and the reaction was carried out for 24 hours at room temperature.

In order to remove the unreacted DVS and residues of inorganic salts from the HA-DVS polymer networks, the samples were washed several times as follow: 3 times in acetone/water $1 / 1(\mathrm{v} / \mathrm{v})$ for 24 hours each and 3 times in deionized water for 24 hours each. Afterwards, the films were dried for 6 hours at $40^{\circ} \mathrm{C}$ and stored in the dark under vacuum until use.

In addition, an uncrosslinked hyaluronan film (HA film) was obtained by a solvent casting process (see Table 2. 1). Briefly, a solution of $10 \mathrm{mg} / \mathrm{mL}$ of hyaluronan sodium salt in deionized water was prepared and shacked for 24 hours. The solution was poured in a petri dish and the water was evaporated for 24 hours at room temperature and subsequently extracted under vacuum for 24 hours. The resulting HA film (1 mm of thickness) was stored in darkness under vacuum until use.

In order to determine some properties of self-polymerized DVS (pDVS) (see Table 2. 1), it was synthesized. $1 \mathrm{~mL}$ of DVS was mixed in $10 \mathrm{~mL} \mathrm{NaOH} 0.2 \mathrm{M}$ for 1 minute and poured in a plastic petri dish $(8.5 \mathrm{~cm}$ of diameter). The reaction was carried out for 24 hours at room temperature. The resulting pDVS was washed twice in de-ionized water for 24 hours each and dried under vacuum for 24 hours. 
Table 2. 1. HA-DVS based materials in which the water is removed by solvent-casting processes.

\begin{tabular}{|c|c|c|c|}
\hline Sample group & $\begin{array}{c}\text { HA:DVS molar } \\
\text { ratio }\end{array}$ & Nomenclature & $\begin{array}{c}\text { Nomenclature of } \\
\text { sample group }\end{array}$ \\
\hline \multirow{3}{*}{$\begin{array}{l}\text { Divinyl sulfone- } \\
\text { crosslinked } \\
\text { hyaluronan } \\
\text { polymer networks }\end{array}$} & $1: 0.6$ & solv_HA:DVS 1:0.6 & \multirow{3}{*}{$\begin{array}{c}\text { solvent-cast HA- } \\
\text { DVS polymer } \\
\text { networks }\end{array}$} \\
\hline & $1: 0.9$ & solv_HA:DVS 1:0.9 & \\
\hline & $1: 1.5$ & solv_HA:DVS 1:1.5 & \\
\hline $\begin{array}{l}\text { Uncrosslinked } \\
\text { hyaluronan film }\end{array}$ & $1: 0$ & HA film & HA film \\
\hline $\begin{array}{l}\text { Self-polymerized } \\
\text { divinyl sulfone }\end{array}$ & $0: 1$ & pDVS & pDVS \\
\hline
\end{tabular}

\subsubsection{Preparation of crosslinked hyaluronan scaffolds}

Porous and interconnected divinyl sulfone-crosslinked hyaluronan scaffolds (HADVS scaffolds) with different HA:DVS molar ratio (see Table 2. 2) were obtained using a template technique. The crosslinking reaction was performed in the interstices of PEMA beads templates to obtain a porous structure. Furthermore, the washing process was slightly modified and additional steps were included to remove the template and dry the samples.

Templates were obtained by a compression and sintering process of PEMA beads, previously sieved between 180-250 $\mu \mathrm{m}$. PEMA beads were compressed in a mould of $15 \times 20 \mathrm{~cm}^{2}$ between two plates in a Gumix T0-250/20 machine, with a power of $7.5 \mathrm{~kW}$ (Gumix S.A.). The process was carried out in two steps: first, the beads were compacted applying a pressure of 100 bars over a sheet of $1 \mathrm{~mm}$ of thickness at $110^{\circ} \mathrm{C}$ for 8 minutes, afterwards the compacted template was sintered applying a pressure of 100 bars over a sheet of $1.5 \mathrm{~mm}$ thickness at $110^{\circ} \mathrm{C}$ for 3.5 minutes. Between each step, the template was cooled until 40 ${ }^{\circ} \mathrm{C}$ by hydraulic refrigeration. The resulting PEMA template consisted in a $2.5 \pm 0.1$ $\mathrm{mm}$ of thickness of $15 \times 20 \mathrm{~cm}^{2}$.

Afterwards, $15 \mathrm{~mL}$ of $\mathrm{HA}$ solution $(30 \mathrm{mg} / \mathrm{mL}$ in $0.2 \mathrm{M} \mathrm{NaOH})$, containing the corresponding amount of DVS were injected into the $5 \times 5 \mathrm{~cm}^{2}$ PEMA templates. The template was filled out with the polymeric solution using vacuum. The excess of the polymeric solution over the template surfaces was removed and the crosslinking reaction was carried out for 24 hours at room temperature. 
After that, PEMA beads template was removed in a Soxhlet extraction apparatus using acetone as solvent. The extraction process was performed for 48 hours. Afterwards, HA-DVS scaffolds were swollen in deionized water for the following 72 hours (the water was replaced every day), freeze-dried and stored in the dark under vacuum conditions until use.

In order to compare HA-DVS scaffolds and bulk polymer networks, different compositions of divinyl sulfone-crosslinked hyaluronan polymer networks (WI_HA-DVS samples) were prepared (see Table 2. 2). In this case, after the reaction time, the post-processes applied were similar to those employed for scaffolds .

Table 2. 2. HA-DVS based materials in which water was removed by a phase inversion process.

\begin{tabular}{cccc}
\hline Sample group & $\begin{array}{c}\text { molar ratio } \\
\text { HA:DVS }\end{array}$ & Nomenclature & $\begin{array}{c}\text { Nomenclature of } \\
\text { sample group }\end{array}$ \\
\hline $\begin{array}{c}\text { Divinyl sulfone- } \\
\text { crosslinked } \\
\text { hyaluronan } \\
\text { scaffolds }\end{array}$ & $1: 0.6$ & scaff_HA:DVS 1:0.6 & \\
\hline $\begin{array}{c}\text { Divinyl sulfone- } \\
\text { crosslinked } \\
\text { hyaluronan }\end{array}$ & $1: 0.9$ & scaff_HA:DVS 1:0.9 & HA-DVS scaffolds \\
polymer networks & $1: 1.5$ & scaff_HA:DVS 1:1.5 & \\
\hline
\end{tabular}

On the other hand, HA-DVS polymer networks using a HA:DVS molar ratio of 1:0.9 (lyo_HA:DVS 1:0.9) were prepared in a similar way than those described above, but in this case the water was removed by a freeze-drying process after the crosslinking reaction. Then, the dry samples were purified employing similar washing steps than those described above and dried by another freeze-drying process. These samples were exclusively employed in the degradation study described in the corresponding section. 


\subsubsection{Synthesis of methacrylated hyaluronan}

Methacrylated hyaluronan (MeHA) was synthesized by the addition of methacrylic anhydride (20-fold excess) to a solution of $50 \mathrm{mg} / \mathrm{mL} \mathrm{HA}$ in $0.2 \mathrm{M}$ $\mathrm{NaOH}$. Briefly, $1.25 \mathrm{~g}$ of hyaluronan sodium salt was dissolved in $25 \mathrm{~mL}$ of $0.2 \mathrm{M}$ $\mathrm{NaOH}$ and mixed in a shaker for 24 hours. The solution was then transferred into a reactor balloon and stirred for $30 \mathrm{~min}$ at $300 \mathrm{rpm}$ under nitrogen atmosphere. Afterwards, $10 \mathrm{~mL}$ of MA was added dropwise and the reaction was carried out in nitrogen atmosphere for 24 hours at room temperature.

The purification process was aimed to remove remaining methacrylic acid, unreacted MA and salts. After the reaction, the solution was precipitated in acetone and solved in deionized water three times to remove undesired products. The resulting solution was filtered employing nylon fabrics with a mesh size of $120 \mu \mathrm{m}$ and the filtered product was dialyzed (MW cutoff $12 \mathrm{kDa}$ ) against deionized water for 3 days. The MeHA product was frozen at $-80^{\circ} \mathrm{C}$ for 4 hours and lyophilized for 3 days. Afterwards the dried product was stored in the dark under vacuum conditions until use.

\subsubsection{Synthesis of poly(methacrylated hyaluronan-co-ethyl acrylate) copolymer networks}

The poly(methacrylated hyaluronan-co-ethyl acrylate) copolymer networks, poly(MeHA-co-EA) copolymer networks, were obtained by free radical copolymerization in a solution of DMF/0.15 M PBS $1 / 1(\mathrm{v} / \mathrm{v})$, using different weight ratios of MeHA to EA (see Table 2. 3). Constant 5 wt \% of crosslinking agent (N,N'-methylenebisacrylamide, MBAA) and $1 \mathrm{wt} \%$ of initiator (potassium persulfate, $\mathrm{K}_{2} \mathrm{~S}_{2} \mathrm{O}_{8}$ ) with respect to the total weight of monomers (HA monomeric unit and EA) were employed. The polymerization reaction was carried out at 60 ${ }^{\circ} \mathrm{C}$ for 24 hours.

After the copolymerization reaction, poly(MeHA-co-EA) copolymer networks were unmoulded carefully. In order to remove the low molecular weight substances and unreacted monomer from the reaction, copolymer networks were washed 5 times in acetone/water $1 / 1(\mathrm{v} / \mathrm{v})$ for 24 hours each and equilibrated in deionized water for 24 hours. Afterwards, poly(MeHA-co-EA) copolymer networks (1-mm thickness) were lyophilized and stored under vacuum until use. 
Table 2. 3. Compositions obtained in the copolymerization of poly(MeHA-co-EA) copolymer networks.

\begin{tabular}{|c|c|c|c|c|}
\hline Sample group & $\begin{array}{c}\text { MeHA:EA } \\
\text { weight } \\
\text { ratio }\end{array}$ & $\begin{array}{c}\text { EA } \\
\text { weight } \\
\text { fraction } \\
\left(W_{E A}\right)\end{array}$ & Nomenclature & $\begin{array}{l}\text { Nomenclature of } \\
\text { sample group }\end{array}$ \\
\hline \multirow{8}{*}{$\begin{array}{c}\text { poly(methacrylated } \\
\text { hyaluronan-co- } \\
\text { ethyl acrylate) } \\
\text { copolymer } \\
\text { networks }\end{array}$} & $1: 0$ & 0 & MeHA:PEA 1:0 & \multirow{8}{*}{$\begin{array}{l}\text { poly(MeHA-co- } \\
\text { EA) copolymer } \\
\text { networks }\end{array}$} \\
\hline & $1: 0.25$ & 0.2 & MeHA:PEA 1:0.25 & \\
\hline & $1: 0.5$ & 0.3 & MeHA:PEA 1:0.5 & \\
\hline & $1: 1$ & 0.5 & MeHA:PEA 1:1 & \\
\hline & $1: 2$ & 0.7 & MeHA:PEA 1:2 & \\
\hline & $1: 5$ & 0.8 & MeHA:PEA 1:5 & \\
\hline & $1: 10$ & 0.9 & MeHA:PEA 1:10 & \\
\hline & $0: 1$ & 1 & PEA & \\
\hline
\end{tabular}




\subsection{Material characterization}

\section{$\checkmark$ Fourier transformed infrared spectroscopy (FTIR)}

Attenuated total reflectance Fourier transformed infrared spectroscopy (ATRFTIR) spectra of solvent-cast HA-DVS polymer networks, HA film and poly(MeHA-co-EA) copolymer networks were recorded by a Thermo Nicolet Nexus FTIR, controlled by OMNIC software on a spectrophotometer (FTIR Thermo Nicolet Nexus). The different spectra were obtained by averaging 64 scans in the range of $700-4000 \mathrm{~cm}^{-1}$ with a resolution of $16 \mathrm{~cm}^{-1}$.

Smart diffuse reflectance Fourier transform infrared spectroscopy (SDR-FTIR) spectra of original HA and HA-DVS scaffolds, WI_HA-DVS samples and MeHA were recorded by the same spectrophotometer described above. Samples were prepared by mixing $1 \mathrm{wt} \%$ of sample with potassium bromide. The spectra were obtained by averaging 64 scans in the range between 700 and $4000 \mathrm{~cm}^{-1}$ with a resolution of $16 \mathrm{~cm}^{-1}$.

\section{$\checkmark$ Elemental analysis CHNS}

The atomic content of carbon, hydrogen, nitrogen and sulphur in the solvent-cast HA-DVS polymer networks and HA-DVS scaffolds was determined using elemental analysis CHNS with an AE1110 CHNS equipment. The method consisted in a Dynamic Flash Combustion process: $1 \mathrm{mg}$ of sample is heated at $1000^{\circ} \mathrm{C}$ with a constant flow of argon stream and the combustion gas mixture is driven through an oxidation catalyst $\left(\mathrm{WO}_{3}\right)$ zone, then though a reduction copper catalyst. The combustion and catalytic reactions yield four different final species: $\mathrm{N}_{2}, \mathrm{CO}_{2}, \mathrm{H}_{2} \mathrm{O}$ and $\mathrm{SO}_{2}$. The gas mixture was eluted and separated by a Porapack PQS column and subsequently detected by a thermal conductivity detector (TCD). The native HA was employed as negative control of sulphur content and each sample was measured by duplicate.

\section{$\checkmark$ Nuclear magnetic resonance ('H-NMR)}

The degree of methacrylation was determined by ${ }^{1} \mathrm{H}-\mathrm{NMR}$ spectroscopy. The ${ }^{1} \mathrm{H}$ NMR spectra were recorded in a Bruker AV-300 at $300 \mathrm{MHz}$ spectrometer. Solutions of both original $\mathrm{HA}$ and $\mathrm{MeHA}$ were prepared at a concentration of 0.3 $\%(\mathrm{w} / \mathrm{w})$ using $0.15 \mathrm{M}$ of $\mathrm{CaCl}_{2}$ in $\mathrm{D}_{2} \mathrm{O}(\delta 4.69)$ as solvent and tetramethylsilane as internal standard. All the chemical shifts for resonance signals are reported in parts per millions (ppm).

${ }^{1} \mathrm{H}$-NMR spectrum of methacrylated $\mathrm{HA}\left(300 \mathrm{MHz}, \mathrm{D}_{2} \mathrm{O}\right.$ with $\left.0.15 \mathrm{M} \mathrm{CaCl}_{2}\right)$ : $\delta$ $1.86\left(\mathrm{~m}, 3 \mathrm{H},-\mathrm{CO}-\mathrm{C}\left(\mathrm{CH}_{3}\right)=\mathrm{CH}_{2}\right.$ belonging to the methacrylate group $), 1.93(\mathrm{~m}$, 
$3 \mathrm{H},-\mathrm{NH}-\mathrm{CO}-\mathrm{CH}_{3}$ from the $\left.\mathrm{HA}\right)$, and 5.66 and $6.29\left(2 \mathrm{~s}, 2 \mathrm{H},-\mathrm{CO}-\mathrm{C}\left(\mathrm{CH}_{3}\right)=\mathrm{CH}_{2}\right.$ belonging to the methacrylate group). Other protons from the HA backbone without practical importance are sited at $\delta$ 3.25-3.72 $(\mathrm{m})$ and 4.39-4.48 $(\mathrm{m})$. The degree of methacrylation (methacrylate groups per HA disaccharide unit) was determined comparing the integrated intensities of the methacrylate protons in MeHA $(\delta 1.86,5.66$ and 6.29) and the methyl protons within the HA acetamide (ठ 1.93).

\section{$\checkmark$ Swelling experiments}

The swelling kinetics was determined gravimetrically using an analytical balance with precision $10^{-5} \mathrm{~g}$ (METLER TOLEDO). The HA-DVS polymer networks were cut in discs of $6 \mathrm{~mm}$ of diameter $\times 1.8 \mathrm{~mm}$ of thickness after the crosslinking reaction. After drying the discs, swelling kinetics was evaluated by immersing the xerogels (dry discs) in different media: controlled $\mathrm{pH}(3,7$ and 8 at ionic strength of $1 \mathrm{M})$, controlled ionic strengths $(0.5,1$ and $2 \mathrm{M}$ at pH 7) and $0.15 \mathrm{M}$ PBS, all them at room temperature. Swelling kinetics was evaluated weighing the swollen samples at different time points. The swelling ratio was estimated at each time according to:

$S W=\frac{w_{s}-W_{d}}{w_{d}}$

[Equation 2. 1]

where, $S W$ represent the weight ratio of water to xerogel; $w_{d}$ and $w_{s}$ are the mass of xerogel and swollen samples at each time, respectively. Each test was carried out by triplicate.

In addition, the equilibrium water content, EWC, which is the swelling ratio in equilibrium, was determined in the solvent-cast HA-DVS polymer networks using the different media described above. Moreover, the EWC was determined in the WI_HA-DVS samples, HA-DVS scaffolds and poly(MeHA-co-EA) copolymer networks immersed in deionized water and $0.15 \mathrm{M}$ PBS. The swollen equilibrium of samples was considered reached after three days in immersion. The WI_HADVS samples and HA-DVS scaffolds were cut in cylindrical shape of $6 \mathrm{~mm}$ of diameter $\times 2 \mathrm{~mm}$ of thickness and poly(MeHA-co-EA) in discs of $6 \mathrm{~mm}$ of diameter $\times 1 \mathrm{~mm}$ of thickness. Each test was carried out by triplicate. 
$\checkmark$ Volumetric swelling

The polymer networks in swollen stage undergo changes in their network parameters. Therefore, differences on discs geometry and volume by measuring thickness and diameter of dry and at equilibrium swollen of solvent-cast HA-DVS polymer networks were determined. The employed media were analogous to those of the swelling experiments. Each measurement was repeated three times for each composition.

The HA volume fraction $(\Phi)$ was determined as follows:

$\Phi=\frac{V_{d}}{V_{s}}=\frac{1}{Q}$

[Equation 2. 2]

where, $Q$ is the reciprocal of $\mathrm{HA}$ volume fraction; $V_{d}$ and $V_{s}$ are the volumes of the dry and swollen network, respectively.

On the other hand, $Q$ can be calculated according to:

$\mathrm{Q}=\frac{\rho_{\mathrm{HA}}^{-1}+E W C \cdot v_{\mathrm{H}_{2} \mathrm{O}}}{\rho_{\mathrm{HA}}^{-1}} \quad$ [Equation 2. 3]

where, $\rho_{\mathrm{HA}}$ is the density of $\mathrm{HA}$ experimentally determined and $\mathrm{v}_{\mathrm{H}_{2} \mathrm{O}}$ is the specific volume of water $\left(1 \mathrm{~cm}^{3} / \mathrm{g}\right)$.

if the swelling is isotropic, the swollen network will show a deformation respect to the dry one with equal stretching ratios: $\lambda_{x}=\lambda_{y}=\lambda_{z}=\Lambda$. Therefore, the isotropic $Q$ (Qisotropic) can be calculated by:

$Q_{\text {isotropic }}=\left(\frac{d_{s}}{d_{d}}\right)^{3}=\Lambda^{3}$

[Equation 2. 4]

where $d_{s}$ and $d_{d}$ are de diameters of the swollen and dry samples, respectively.

Similar values between Qisotropic and $Q$ indicate an isotropic swelling of the networks. 


\section{Density}

Density of solvent-cast HA-DVS polymer networks and HA film was determined gravimetrically by measuring the weight of dried samples in air and immersed in octane according to Equation 2. 5. A Mettler Toledo AX205 balance with a density accessory kit with a sensitivity of $10^{-5} \mathrm{~g}$ was used. Density, $\rho$, was determined as follows:

$\rho=\rho_{\text {octane }}\left(\frac{w_{a}}{w_{a}-w_{\text {octane }}}\right)$

[Equation 2. 5]

where, poctane is the density of octane at $25 \stackrel{\circ}{\circ} \mathrm{C}\left(0.703 \mathrm{~g} / \mathrm{cm}^{3}\right) ; w_{a}$ and $w_{\text {octane }}$ are the mass of each sample in air and octane, respectively.

Measurements were obtained by triplicate for each composition.

\section{$\checkmark$ Porosity determination}

Porosity and macroporosity of HA-DVS scaffolds were determined gravimetrically by two different procedures. The porosity (large and small pores) was calculated by measuring weights of dry scaffolds and the octane filling their interstices, while macroporisity (large pores) was determined from scaffolds swollen in water and weighting the amount of octane displacing water. In both cases, the octane was introduced in the scaffolds applying vacuum.

Porosity, $\pi_{p}$, and macroporosity, $\pi_{m}$, can be calculated from the ratio between the volume of small pores, $V_{\text {pores }}$, or large pores, $V_{\text {macropores }}$, respectively, and the total volume of the sample, $V_{t}$, as follows:

$\pi_{p / m}=\frac{\mathrm{V}_{\text {pores } / \text { macropores }}}{\mathrm{V}_{\mathrm{t}}}=\frac{\mathrm{V}_{\text {pores } / \text { macropores }}}{\mathrm{V}_{\mathrm{HA}}+\mathrm{V}_{\text {pores } / \text { macropores }}}$

[Equation 2. 6]

where, each term of the equation can be calculated by:

$$
\begin{array}{ll}
V_{\text {pores }}=m_{\text {octane }} \cdot V_{\text {octane }} & {[\text { Equation 2. 7] }} \\
V_{H A}=m_{H A} \cdot \rho_{H A}^{-1} & {[\text { Equation 2. 8] }}
\end{array}
$$


where, $m_{\text {octane }}$ is the mass of the introduced octane, $v_{\text {octane }}$ is the specific volume of octane $\left(1.422 \mathrm{~cm}^{3} / \mathrm{g}\right), V_{H A}$ is the volume of $\mathrm{HA}$ in the sample, $m_{H A}$ is the mass of dry $\mathrm{HA}$, and $\rho_{H A}$ is the density of $\mathrm{HA}$ (derived from calculations described above).

All measurements were carried out by triplicate.

\section{$\checkmark$ Compression tests}

Compression tests were conducted in a Seiko EXSTAR TMA/ss6000 Dilatometer (Seiko Instruments Inc.) in the compression mode. Compression of solvent-cast HA-DVS polymer networks was performed at room temperature, under uniaxial and unconfined compression and in their equilibrium swollen state in different media: three $\mathrm{pH}(3,7$ and 8$)$ at an ionic strength of $1 \mathrm{M}$, three ionic strengths $(0.5$, 1 and $2 \mathrm{M}$ ) at $\mathrm{pH} 7$ and $0.15 \mathrm{M}$ PBS. Besides, compression tests on HA-DVS scaffolds, WI_HA-DVS samples and poly(MeHA-co-EA) copolymer networks were performed in $0.15 \mathrm{M}$ PBS. Samples (solvent-cast HA-DVS polymer networks, HA-DVS scaffolds, WI_HA-DVS samples and poly(MeHA-co-EA) copolymer networks) were cylindrical shaped with a diameter of 5-6 $\mathrm{mm}$ and the height was measured by the apparatus applying a load of $5 \mathrm{mN}$. Solvent-cast HADVS polymer networks were compressed with forces between 5 and $3500 \mathrm{mN}$ and conducted at $200 \mathrm{mN} / \mathrm{min}$. WI_HA-DVS samples, HA-DVS scaffolds and poly(MeHA-co-EA) copolymer networks were tested with forces between 5 and $150 \mathrm{mN}$ and conducted at $5 \mathrm{mN} / \mathrm{min}$. Furthermore, brain tissue from 5 days postnatal Wistar rats was tested using similar conditions than those used in the HA-DVS scaffolds. Each test was repeated three times.

Stress-strain curves were obtained for each material conversing the compressed thickness, $\Delta e$, in strain, $\varepsilon$, and force, $F$, in tension, $\sigma$, respectively, as follows:

$\sigma=\frac{\mathrm{F}}{\mathrm{A}} ; \varepsilon=\frac{\Delta \mathrm{e}}{\mathrm{e}_{0}} \quad$ [Equation 2.9]

where, $e_{0}$ and $A$ are the initial thickness and cross-section of the sample, respectively.

Stress-strain curves permit to determine several parameters, which are related with the mechanical properties of a material. The initial modulus, $E$ or $E_{0}$, was obtained using the curve slope at the unitary strain range between 0 and 0.1 . A modulus at advanced compression, $E_{f}$, was calculated in the tension range from 
3 to $3.5 \mathrm{kPa}$. The $E_{f}$ slope was interpolated until section with the strain axis to determine a unitary strain at advanced compression, $\varepsilon_{f}$.

\section{$\checkmark$ Dynamic mechanical thermal analysis (DMTA)}

DMTA analysis of dry poly(MeHA-co-EA) copolymer networks was performed in a dilatometer Seiko TMA/SS6000 at a frequency of $1 \mathrm{~Hz}$ in the tension mode. The temperature dependence from the storage and loss module was recorded in the temperature range from -60 to $60{ }^{\circ} \mathrm{C}$ at a rate of $2{ }^{\circ} \mathrm{C} / \mathrm{min}$. Samples tested were prismatic $\left(2.5 \times 0.5 \times 0.1 \mathrm{~cm}^{3}\right)$.

The storage moduli of each sample was normalized dividing by the storage moduli at $-60{ }^{\circ} \mathrm{C}, E^{\prime} / E^{\prime}-60^{\circ} \mathrm{C}$. This ratio was fitted to the predictions from the Takayanagi's mechanical block model for bi-phasic systems with the aim to determine phase distribution in the copolymer networks [360]. The model takes into account a disperse phase, of volume fraction $\varphi$, working in parallel (deforms with the same strain) with a volume fraction of the continuous phase, $(1-\lambda) \cdot(1-\varphi)$, and both work in series (deform with the same stress) with a volume fraction of the continuous phase $\lambda \cdot(1-\varphi)$.

\section{$\checkmark$ Thermogravimetric analysis (TGA)}

Thermal degradation of solvent-cast HA-DVS polymer networks, HA-DVS scaffolds and poly(MeHA-co-EA) copolymer networks was performed using a TGA thermogravimetric analyser (TA-Instrument SDT-Q600 system). Samples (5-10 mg weight) were placed on the balance and the temperature rose from room temperature to $700{ }^{\circ} \mathrm{C}$ at a heating rate of $10^{\circ} \mathrm{C} / \mathrm{min}$, under a positive flow of nitrogen. The mass of the sample pan was continuously monitored as a function of temperature. The assay was performed by duplicate.

\section{$\checkmark$ Differential Scanning Calorimetry (DSC)}

Differential scanning calorimetry (DSC) of poly(MeHA-co-EA) copolymer networks was performed in a DSC device (Mettler Toledo DSC 823e). Temperature of the instrument was calibrated employing indium and zinc. Assays were carried out in the temperature range from -80 to $30^{\circ} \mathrm{C}$ at a rate of $10{ }^{\circ} \mathrm{C} / \mathrm{min}$. The samples were analysed after the freeze-drying process and after a thermal treatment $(150 \stackrel{\circ}{\circ}$ for 4 hours). 
$\checkmark$ Morphological characterization by scanning electron microscopy (SEM).

The morphology of HA-DVS scaffolds and poly(MeHA-co-EA) copolymer networks was analysed using a JEOL JSM 5410 scanning electron microscope (SEM), at an accelerating voltage of $10 \mathrm{kV}$. The cross-sections, obtained by cryogenic rupture under liquid nitrogen, and surfaces of the different samples were analysed. Samples were mounted on copper stubs and gold coated before observation.

\section{$\checkmark$ In vitro degradation}

In order to determine the enzymatic degradation of HA in vitro, a solution containing hyaluronidase from bovine testes (provided by Aldrich) was employed. The degradation was studied on solv_HA:DVS 1:0.9, WI_HA-DVS samples, HADVS scaffolds and on three compositions of poly(MeHA-co-EA) copolymer networks $(0,1$ and 10$)$ with a diameter of $5 \mathrm{~mm}$. The enzymatic digestion was carried out at $37 \stackrel{\circ}{\circ} \mathrm{C}$ immersing samples in $1 \mathrm{~mL} 0.15$ M PBS containing 10 units $/ \mathrm{mL}$ of enzyme and sodium azide $0.05 \%$. The degradation was achieved at different time points and enzymatic solution was replaced every 2 weeks. After each time point, the samples were rinsed with deionized water and the enzyme in the supernatants was deactivated by boiling each vial into a water bath for 5 minutes. All samples and supernatants were stored at $-20{ }^{\circ} \mathrm{C}$ until characterization. The degraded samples were subjected to a freeze-drying process before characterization. Each sample was studied by triplicate and samples immersed in $0.15 \mathrm{M}$ PBS for 3 and 56 days were taken as controls.

The weight loss was determined gravimetrically weighting the dry samples before and after the enzymatic degradation. The molecular weight distribution of the degrading chains contained in the supernatants was determined, fitting the fraction of each specie, $w_{i}$, of molecular weight $M_{i}$, by gel permeation chromatography (GPC) using a Waters model 1525 equipment. The chromatographic device is composed of a binary HPLC pump for gel permeation chromatography, an autosampler, a module that includes a column oven and a refractive index detector. The column oven consists in four $7.8 \mathrm{~mm} \times 300 \mathrm{~mm}$ porous bed GPC columns of Ultrahydroge ${ }^{\mathrm{TM}}$, which are connected in series and contain pore sizes of $1000,500,250$ and $120 \mu \mathrm{m}$. $100 \mu \mathrm{L}$ of supernatant were injected each 80 minutes employing a flow of $0.15 \mathrm{M} \mathrm{NaCl}$ containing sodium azide $0.05 \%$ at $0.8 \mathrm{~mL} / \mathrm{min}$. The calibration curve was plotted using PEO/PEG standards from 100 to $8.3 \times 10^{5} \mathrm{Da}$ and a solution of $1 \mathrm{mg} / \mathrm{mL}$ in $0.15 \mathrm{M}$ PBS of original HA. Each supernatant was studied by duplicate. On the other hand, the effect of degradation over properties and morphology of each sample was studied determining EWC in $0.15 \mathrm{M}$ PBS and morphology by SEM and the remaining 
MeHA component after the degradation was analysed by TGA, as mentioned earlier.

Furthermore, the degree of hydrolysis of original $\mathrm{HA}$ in solutions of $0.2 \mathrm{M} \mathrm{NaOH}$ was determined by GPC. Two HA solutions of 30 and $50 \mathrm{mg} / \mathrm{mL}$ in $0.2 \mathrm{M} \mathrm{NaOH}$ were prepared and shacked for 24 hours. Afterwards, HA solutions were neutralized with $0.2 \mathrm{M} \mathrm{HCl}$ and the $\mathrm{HA}$ concentration was adjusted to $1 \mathrm{mg} / \mathrm{mL}$ in a final solution of $0.15 \mathrm{M} \mathrm{NaCl}$. Additionally, the molecular weight of the MeHA macromer was determined from a solution of $1 \mathrm{mg} / \mathrm{mL}$ in $0.15 \mathrm{M} \mathrm{NaCl}$.

\section{$\checkmark$ Statistical analyses}

The significant differences of the experimental data were determined by statistical analysis using a one-way ANOVA with a Tukey multiple range test by the software Statgraphics. The test was performed at $95 \%$ of confidence ( $p$-value < 0.05).

The data belonging to a homogeneous group (without significant differences) were indicated by a similar numerical value $(1,2,3$ '...) in the corresponding figure. These numerical values correlate the average of the data in an ascendant manner and are plotted in the figure considering the relative position of the experimental data. 


\subsection{Preparation of materials for cultures and viability assays}

\section{$\checkmark$ Preparation of materials and extracts}

First of all, materials (HA-DVS scaffolds, MeHA:PEA 1:1 samples and PEA) were conditioned for cell cultures using 70/30 (v/v) ethanol/water mixtures and UV light. The disinfection process was performed as follows: samples were washed three times in ethanol/water 70/30 (v/v) (two times for 30 minutes and the last one overnight); after that, the materials were rehydrated in deionized water; and subsequently the water was replaced by Dulbecco's Phosphate Buffered Saline (DPBS) and exposed under UV light for 30 minutes; finally samples were incubated in ECBM for 24 hours.

The extracts, the ECBM medium in contact with materials from the conditioning stage described above, were collected for each material. The extracts were employed to study the viability and proliferation of SH-SY5Y, U373 and hCMEC/D3 cell lines. Besides, the inflammatory effect on the hCMEC/D3 cell line as a consequence of being in contact with material extracts was determined.

\section{$\checkmark$ Cell cultures}

96-well plates were used to performed cell culture studies, coating wells with 10 $\mu \mathrm{g} / \mathrm{mL}$ fibronectin (FN) overnight before use. SH-SY5Y and hCMEC/D3 cell lines at a density of 35000 cells per well were seeded, on the other hand in the case of U373 cell line, 15000 cells per well were seeded. All cells populations were incubated in ECBM for 48 hours, and afterwards the medium was replaced by the corresponding extract and cells were incubated again for 4 and 24 hours. Cell viability, proliferation and inflammation were addressed using pure extract and three different extract dilutions $(1 / 2,1 / 4$ and $1 / 8 \mathrm{v} / \mathrm{v})$. Each experience was performed by triplicate.

$\checkmark$ Cytotoxicity tests: MTS, lactate dehydrogenase (LDH) release and Crystal violet assays

The cytotoxicity induced on each cell type by the material extracts were determined by MTS assay, the lactate dehydrogenase (LDH) release assay and the crystal violet staining. The different cell types were cultured for 4 and 24 hours in contact with the material extracts and dilutions as described above. Three replicates for each medium and cell type were performed.

The viability of cell cultures in contact with extracts was determined employing a 3-(4,5-dimethylthiazol-2-yl)-5-(3-carboxymethoxyphenyl)-2-(4-sulfopheny)-2Htetrazolium (MTS) assay (CellTiter 96 Aqueous One Solution cell proliferation 
assay kit; Promega). A soluble formazan product was synthesized by mitochondrial activity of MTS, and the quantification of this soluble product was proportional to living cells. After each culture period, media from 96-well plates were removed and stored in new plates, and cells were incubated with MTS diluted in ECBM (1:5) for 45 minutes. $100 \mu \mathrm{L}$ of incubated MTS media was added in a new plate and the absorbance was read at $490 \mathrm{~nm}$. Cell populations cultured in ECBM for 4 hours were taken as positive control (set as the $100 \%$ of viability) and cells cultured in ECBM for 4 hours and incubated with $1 \mu \mathrm{g} / \mathrm{mL}$ of lyase for 45 minutes were taken as negative control.

Cell apoptosis or necrosis was quantified by the lactate dehydrogenase (LDH) assay (CytoTox 96 non-radioactive cytotoxicity assay; Promega). The LDH is a soluble cytosolic enzyme that is released into the culture medium following loss of membrane integrity resulting from both apoptosis and necrosis. LDH release into the medium was detected using the LDH assay kit as recommended by the manufacturer. $50 \mu \mathrm{L}$ of stored cell cultured medium mentioned above were put in a new 96-well plate, $50 \mu \mathrm{L}$ of substrate from LDH assay kit were added and the mixture was incubated for 30 minutes. The reaction was stopped by adding stop solution $\left(\mathrm{HCl} / \mathrm{H}_{2} \mathrm{OSS}_{4}\right.$ mixture) and the absorbance was measured at $490 \mathrm{~nm}$. The LDH content of supernatants of cell populations cultured in ECBM for 4 hours was employed as negative control and the one of cells cultured in ECBM for 24 hours and incubated with $1 \mu \mathrm{g} / \mathrm{mL}$ of lyase for 45 minutes were taken as positive control (set as the $100 \%$ of apoptosis).

After the MTS assay, cells were fixed with a methanol/ethanol mixture 2/1 (v/v) for 5 minutes and the viability was tested by the crystal violet assay. The crystal violet is a triphenylmethane dye (4-[(4-dimethylaminophenyl)-phenyl-methyl]$\mathrm{N}, \mathrm{N}$-dimethyl-aniline), which has a high affinity for the highly acidic chromatin of mitotic cells. The method consisted in adding $100 \mu \mathrm{L}$ of $0.1 \%$ crystal violet in fixed cells and subsequent incubation for 30 minutes. After the incubation period, the crystal violet was removed and the plate was rinsed with water and dried overnight at room temperature. The next day, $100 \mu \mathrm{L}$ of $33 \%$ acetic acid were added and incubated for 15 minutes at $37^{\circ} \mathrm{C}$. Afterwards, $90 \mu \mathrm{L}$ of this solution were poured in a new plate and the absorbance was measured at $590 \mathrm{~nm}$. Cell populations cultured with ECBM for 4 hours were employed as positive control (set as the $100 \%$ of viability) and cells cultured in ECBM for 24 hours and incubated with $1 \mu \mathrm{g} / \mathrm{mL}$ of lyase for 45 minutes were taken as negative control.

\section{$\checkmark$ Proliferation assay}

Cell proliferation was evaluated by the detection of the Ki-67 protein, exclusively detected during the interphase and mitosis of proliferating cells. The different cell populations were cultured for 4 and 24 hours in contact with the material extracts 
and dilutions as described above. Three replicates for each medium and cell type were performed.

First, the fixed cells were permeabilized with $0.1 \%$ Triton-X-100 in PBS for 5 minutes at room temperature, and, after several rinses, the unspecific sites of cells were blocked with a block solution, containing $1 \% \mathrm{H}_{2} \mathrm{O}_{2}$, incubated for 1 hour. Afterwards, cells were incubated with a block solution containing $1 \mu \mathrm{g} / \mathrm{mL}$ primary antibody (Ki-67, monoclonal mouse anti-human Ki-67 antigen clone MIB1. Dako) for 45 minutes at $37^{\circ} \mathrm{C}$. After several washing steps, cells were incubated in block solution containing secondary antibody, igG1 peroxidase (polyclonal rabbit anti-mouse immunoglobulins HRP, Dako), at a dilution 1:400 for 45 minutes at $37^{\circ} \mathrm{C}$. Subsequently, a solution containing $50 \mathrm{~mL}$ of $0.1 \mathrm{M}$ citric buffer, $20 \mu \mathrm{L}$ of $30 \% \mathrm{H}_{2} \mathrm{O}_{2}$ and an o-Phenylenediamine dihydrochloride tablet was added and incubated for 15 minutes at $37^{\circ} \mathrm{C}$. Finally, $160 \mu \mathrm{L}$ of this solution were added to a new plate containing $50 \mu \mathrm{L}$ of $3 \mathrm{M} \mathrm{HCl}$ and the absorbance was measured at $492 \mathrm{~nm}$. The cell populations cultured in ECBM for 4 hours were used as positive control (set as the $100 \%$ of proliferation) and cells cultured in ECBM for 24 hours and incubated with $1 \mu \mathrm{g} / \mathrm{mL}$ of lyase for 45 minutes were taken as negative control.

\section{$\checkmark$ Inflammatory assay}

The E-selectin expression was studied with the aim to determine inflammatory effects in the hCMEC/D3 cell line exposed to the different extracts for 4 and 24 hours. The E-selectin is expressed exclusively in endothelial cells and is activated by cytokines, playing an important role in the inflammatory process. Three replicates for each medium and cell type were performed.

At the beginning, the fixed cells were blocked by adding a block solution containing $1 \% \mathrm{H}_{2} \mathrm{O}_{2}$ at $37 \stackrel{\circ}{\circ} \mathrm{C}$ for 60 minutes. After blocking, the primary antibody, E-selectin (Anti-Human CD62E; eBioscience), was incubated in block solution at a dilution $1: 2000$ for 45 minutes at $37^{\circ} \mathrm{C}$. Afterwards, the secondary antibody, anti-mouse IgG biotinylated (RPN 1177; GE Healthcare Life Sciences), was incubated in block solution at a dilution 1:1000 for 45 minutes at $37{ }^{\circ} \mathrm{C}$. The signal of E-selectin expression was enhanced adding a biotinylated streptavidin complex (GE Healthcare Life Sciences) in the block solution at a dilution 1:1000 for 60 minutes at $37^{\circ} \mathrm{C}$. Subsequently, a solution containing $50 \mathrm{~mL}$ of $0.1 \mathrm{M}$ citric buffer, $20 \mu \mathrm{L}$ of $30 \% \mathrm{H}_{2} \mathrm{O}_{2}$ and an o-Phenylenediamine dihydrochloride tablet was added and incubated for 15 minutes at $37^{\circ} \mathrm{C}$. Finally, the product was transferred in a new plate with $3 \mathrm{M} \mathrm{HCl}$ and the absorbance was read at $492 \mathrm{~nm}$. The cell line line cultured in ECBM containing $1 \mu \mathrm{g} / \mathrm{mL}$ of LPS and incubated for 4 hours were considered as positive control (set as the $100 \%$ of inflammation) and the culture in ECBM was taken as negative control. 


\subsection{Colonization studies on scaffolds}

\section{$\checkmark$ Cell seeding on HA-DVS scaffolds}

First of all, HA-DVS scaffolds of $6 \mathrm{~mm}$ of diameter $\times 2 \mathrm{~mm}$ of thickness (scaff_HA:DVS 1:0.6, scaff_HA:DVS 1:0.9 and scaff_HA:DVS 1:1.5) were conditioned following the steps described in section 2.4, next, the scaffolds were coated with $\mathrm{FN}$ at a concentration of $10 \mu \mathrm{g} / \mathrm{mL}$ before seeding.

In all the cultures (see Table 2. 4), the ECBM was employed as culture medium and the density of each cell population was as follows: U373 at 15000 cells per biomaterial, and SH-SY5Y, pBECs and hCMEC/D3 at 35000 cells per biomaterial. Each cell seeding was performed covering the materials with $100 \mu \mathrm{L}$ of ECBM containing the corresponding amount of cells and incubating for 4 hours before medium replacement or next seeding (in the case of co-cultures and triplecultures).

Table 2. 4. Combinations of cell populations for cultures on the HA-DVS scaffolds.

\begin{tabular}{cc}
\hline Culture combination & Cell populations employed in each experiment \\
\hline \multirow{3}{*}{ Monocultures } & SH-SY5Y \\
& U373 \\
& hCMEC/D3 \\
& $\mathrm{pBEC}$ \\
\hline Co-cultures & $\mathrm{SH}-\mathrm{SY} 5 \mathrm{Y}+\mathrm{hCMEC/D3}$ \\
& $\mathrm{U} 373+\mathrm{hCMEC/D3}$ \\
& $\mathrm{SH}-\mathrm{SY} 5 \mathrm{Y}+\mathrm{pBEC}$ \\
$\mathrm{U} 373+\mathrm{pBEC}$ \\
\hline \multirow{2}{*}{ Triple-cultures } & $\mathrm{SH}-\mathrm{SY} 5 \mathrm{H}+\mathrm{U} 373+\mathrm{hCMEC/D3}$ \\
& $\mathrm{SH}-\mathrm{SY} 5 \mathrm{Y}+\mathrm{U} 373+\mathrm{pBEC}$ \\
\hline
\end{tabular}

In order to determine the optimal HA-DVS scaffold composition for cell colonization, each cell population was seeded as monoculture (see Table 2. 4) and incubated for 7 days. After culture, each cell population was immunostained and fluorescence images were taken (see below). The optimal HA-DVS scaffolds for colonization were employed to carry out co-cultures and triple-cultures for 7 and 14 days (Table 2. 4). Moreover, cultures on 96-well plates were employed as controls. In the case of co-cultures, U373 or SH-SY5Y cell lines were first seeded and, after incubating 4 hours and removing medium, the corresponding 
endothelial cell population was seeded. In triple-cultures, U373 and SH-SY5Y cell lines were simultaneously seeded and next the corresponding endothelial cell population was seeded. The ECBM was replaced every two or three days.

In addition to cell cultures on HA-DVS scaffolds, the different cell populations were seeded as monocultures (described in Table 2. 4) and incubated for 7 days onto FN-coated MeHA:PEA 1:0, MeHA:PEA 1:5 and PEA samples using similar conditions.

\section{$\checkmark$ Immunohistochemistry}

The different cell populations were studied by immunohistochemistry employing neuronal, glial and endothelial markers. After each culture, cells were fixed with $3.7 \%$ paraformaldehyde (Merck, Darmstadt, Germany) at room temperature for 15 minutes. Cells were then rinsed three times in $0.1 \mathrm{M} \mathrm{PBS}$, and permeabilized with $0.2 \%$ Triton-X-100 in $0.1 \mathrm{M}$ PBS for 5 minutes. Afterwards, cells were rinsed, and blocked with $1 \%$ of foetal calf serum in $0.1 \mathrm{M}$ PBS (PBSA) at room temperature for 1 hour.

Primary antibodies were diluted in PBSA and incubated at room temperature for 1 hour. Glial fibrillary acidic protein (GFAP) immunoreactivity was employed for U373 cells; however, depending on the culture combination rat anti-GFAP ( $r$ GFAP, monoclonal rat anti-GFAP; Invitrogen) diluted at 1:200 or mouse antiGFAP (m-GFAP, monoclonal mouse anti-GFAP; Sigma) diluted at 1:300 were employed. Neuron-specific class III beta-tubulin, mouse anti-Tuj-1 (Tuj-1, monoclonal mouse anti-Tuj-1; Millipore) diluted at 1:100 was used as a marker for SH-SY5Y cells. Positive cells to the cluster of differentiation 31 were defined as endothelial cells; rat anti-pig CD-31 (r-CD-31, monoclonal rat anti-pig CD-31; R\&Dsystems) diluted at 1:200, and mouse anti-human CD-31 (m-CD-31, monoclonal mouse anti-human CD-31; DAKO) diluted at 1:50 were taken as markers for $\mathrm{pBEC}$ and $\mathrm{hCMEC/D3}$, respectively. After several rinses with PBSA to remove unreacted primary antibodies, secondary ones diluted 1:1000 in PBSA were incubated at room temperature for one hour. The secondary antibodies employed were Alexa Fluor ${ }^{\mathrm{TM}}$ goat anti-mouse IgG 546 (A-11030; Invitrogen), Alexa Fluor ${ }^{\mathrm{TM}}$ goat anti-rat IgG 488 (A-11006; Invitrogen), goat anti-mouse IgG 488 (A-11029; Invitrogen). In addition, cell nuclei were stained with Hoechst diluted in PBS $(1: 10000)$ at room temperature for 5 minutes. Fluorescence images were recorded using a fluorescence microscope (BZ-900, KEYENCE).

The presence of each cell population was determined by immunohistochemical staining in monocultures and co-cultures, but only endothelial cell populations and U373 cell line were stained in triple-cultures (unstained cells were interpreted as SH-SY5Y cell line). Antibody incubations were performed in a different way 
depending on the type of endothelial cells employed in co-cultures and triplecultures. Immunohistochemical staining for pBECs co-cultures and triple-cultures were performed in a single step; the corresponding two primary antibodies ( $\mathrm{m}$ GFAP or Tuj-1 and r-CD-31) were incubated together, followed by the incubation of the two appropriate secondary antibodies. However, the staining process in co-cultures and triple-cultures including hCMEC/D3 cell line were performed in two steps; m-CD-31 antibody was incubated followed by the incubation of the corresponding secondary one, and the process was repeated by incubating the r-GFAP or Tuj-1 antibodies followed by the corresponding secondary ones. 



\section{CHAPTER III}

RESULTS 



\subsection{Crosslinking of hyaluronan with divinyl sulfone}

$\mathrm{HA}$ is the major constituent of the ECM in many tissues, and it is continuously synthesized by cells and degraded by the hyaluronidase activity. Additionally, HA in solution is characterized by its viscoelastic properties with a low consistence. The high degradation rate and solubility in physiological media and poor mechanical properties suppose a limitation for the use of HA as artificial ECM for cell support. For this reason, the scaffolds based on HA are usually derivatives thereof using crosslinking or other chemical modifications. HA can be modified by a broad range of chemical reactions, but only a few HA derivatives are currently employed in clinical applications. For these purposes, one of the most employed HA derivatives is the DVS-crosslinked HA. In this section, the formation of hyaluronan polymer networks crosslinked with DVS (solvent-cast HA-DVS polymer networks) is studied and the resulting hydrogels are characterized.

\subsubsection{Crosslinking of hyaluronan with divinyl sulfone}

The crosslinking of HA with DVS was described in section 2.2.1. Therefore, the crosslinking reaction was performed in presence of $\mathrm{NaOH} 0.2 \mathrm{M}$ resulting in a solution with $\mathrm{pH}$ values between 13 and 14. Furthermore, basic medium provides to the HA solution an acceptable viscosity for an easier handling.

The further addition of DVS into the colourless HA solution becomes it to a yellowish solution in a few seconds, showing a rapid homogenization of the mixture. In order to select the appropriate HA:DVS molar ratio (referred the molar ratio of HA repeat unit to DVS), different amounts of DVS were employed in the development of the HA-DVS polymer networks. On the other hand, the crosslinking reaction of HA using DVS shows low efficiencies and DVS is usually added in molar excess. Thus, in this study DVS was added in amounts below and above a theoretical degree of crosslinking of $100 \%$ (HA:DVS molar ratio 1:0.5). The HA:DVS molar ratios employed were 1:0.2, 1:0.4, 1:0.6, 1:0.9, 1:1.5, 1:1.8 and 1:2. After the crosslinking reaction, the HA-DVS polymer networks show in general a soft, homogeneous and consistent structure with yellowish tones corresponding to the unreacted DVS. However, the HA-DVS polymer networks with molar ratios of $1: 1.8$ and $1: 2$ gave heterogeneous hydrogels including rigid aggregates derived from the self-polymerization of DVS. After the washing process, the HA-DVS polymer networks become colourless indicating the removal of unreacted DVS, whereas the dried samples become transparent and show a vitreous appearance.

The equilibrium water content, EWC, of the HA-DVS polymer networks in $0.15 \mathrm{M}$ PBS was determined (described in section 2.3). The data of EWC in the different 
HA-DVS polymer networks as function of the molar ratio of DVS to HA is plotted in Figure 3. 1.

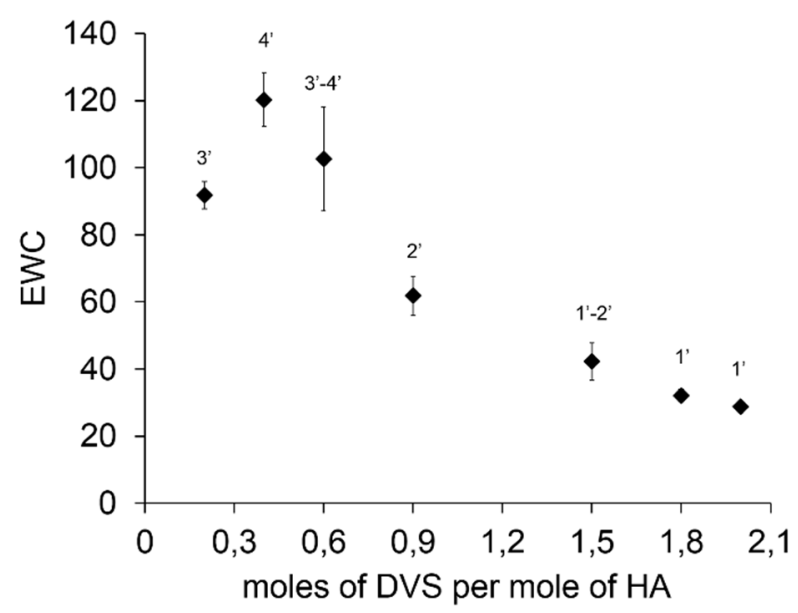

Figure 3. 1. EWC of the HA-DVS polymer networks as function of the molar ratio of DVS to HA. Vertical bars show the standard deviation.

Figure 3. 1 shows that the amount of DVS influences in the value of EWC. In general, the higher amount of DVS, the lower EWC value, ranging from 120 to 30. The value of EWC drastically decreases from molar ratios around a theoretical degree of crosslinking of $100 \%$ (HA:DVS molar ratio 1:0.5) and such value is stabilized for the HA-DVS polymer networks containing larger amounts of DVS. However, the HA-DVS polymer network with a HA:DVS molar ratio of 1:0.2 shows an anomalous lower value than the corresponding one taking into account the curve trend.

On the other hand, the effect of the alkaline medium, employed in the crosslinking reaction, on the hydrolysis of $\mathrm{HA}$ chains was estimated. In order to corroborate this hypothesis, the molecular weight distributions of $\mathrm{HA}$ in a solution of $0.2 \mathrm{M}$ $\mathrm{NaOH}$ and in a solution of $0.15 \mathrm{M} \mathrm{NaCl}$ (described in section 2.3) were compared. After stirring for 24 hours, the solutions were neutralized (in the case of the alkaline medium) and diluted to a final solution of $1 \mathrm{mg} / \mathrm{mL}$ in $0.15 \mathrm{M}$, and then both solutions were passed by GPC. 


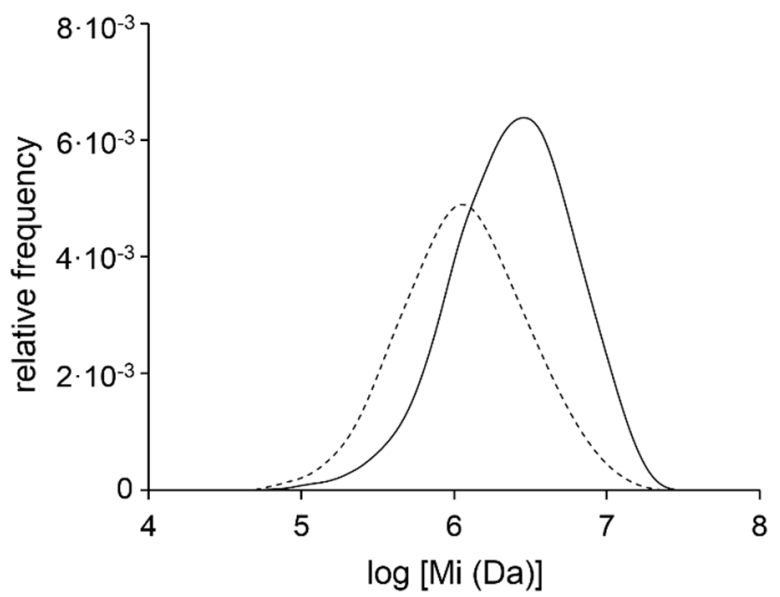

Figure 3. 2. Molecular weight distribution of $\mathrm{HA}$ chains dissolved in $0.15 \mathrm{M} \mathrm{NaCl}$ (solid line) and 0.2 $\mathrm{M} \mathrm{NaOH}$ (dashed line).

The molecular weight distribution of $\mathrm{HA}$ dissolved $0.15 \mathrm{M} \mathrm{NaCl}$ shows an asymmetric and narrow curve predominating chains with lower molecular weights. However, the $\mathrm{HA}$ in $0.2 \mathrm{M} \mathrm{NaOH}$ displayed a more symmetrical distribution showing a reduction in its molecular weight. Experimental data arise that $\mathrm{HA}$ dissolved in $0.15 \mathrm{M} \mathrm{NaCl}$ shows a weight average molecular weight, $M w$, of $1.74 \times 10^{6} \mathrm{Da}$ with a high polydispersity index, $\mathrm{PDI}$, of 4 . Moreover, the HA dissolved in alkaline media leads to a high reduction of the $M w\left(7.25 \times 10^{5} \mathrm{Da}\right)$ and the $P D I$ decreases to values of 3.3 .

\subsubsection{Characterization of crosslinked hyaluronan}

The study of the EWC of the HA-DVS polymer networks with different amount of DVS helped to choose three representative compositions with the aim to carry out a more complete characterization. The HA-DVS polymer networks showing evidences of unreacted chains (molar ratio 1:0.2) and aggregates within the hydrogels (molar ratio 1:1.8 and 1:2) were discarded. Therefore, three intermediate HA-DVS polymer networks with different HA:DVS molar ratio were selected for further studies: 1:0.6, 1:0.9 and 1:1.5. 


\subsubsection{Fourier transformed infrared spectroscopy}

In order to determine the new functional groups in the HA-DVS polymer networks after the crosslinking reaction, the spectra of pDVS, the HA film and the different HA-DVS polymer networks (all them obtained as described in section 2.3) were recorded.

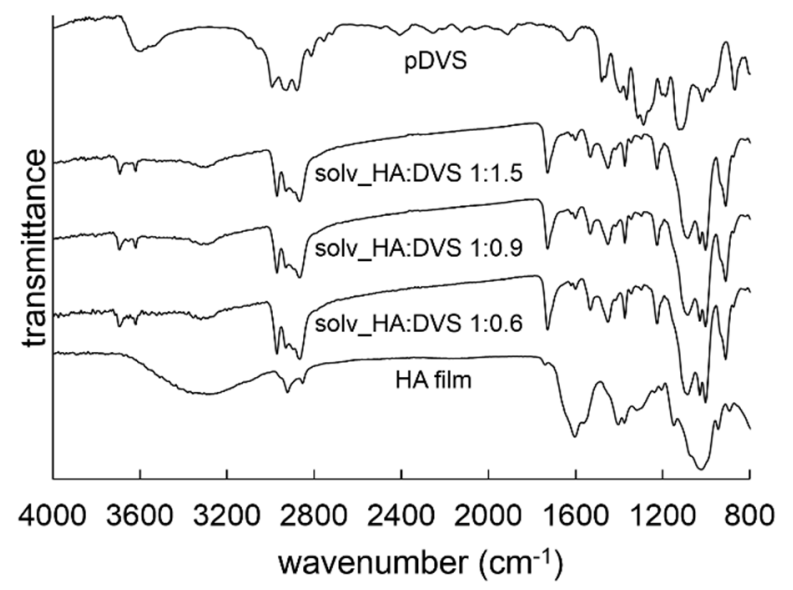

Figure 3. 3. IR spectra of HA film, HA-DVS polymer networks and pDVS.

The spectrum of unmodified HA film shows a broad absorption band between 3500 and $3000 \mathrm{~cm}^{-1}$ characteristic of the overlapping of associated $\mathrm{O}-\mathrm{H}$ and $\mathrm{N}-\mathrm{H}$ stretching vibrations of hydroxyl groups, carboxylic acids and amide groups. The multiple peaks in the range from 3000 to $2800 \mathrm{~cm}^{-1}$ are attributed to the sp3hybridization in carbons. The broad absorption band at $1600 \mathrm{~cm}^{-1}$ corresponds to the superposition of bound $\mathrm{C}=\mathrm{O}$ groups of amides (Amide 1). The double peak between 1500 and $1300 \mathrm{~cm}^{-1}$ is attributed to the $\mathrm{N}-\mathrm{H}$ bending of amide groups (Amide II) and $\mathrm{O}-\mathrm{H}$ bending in hydroxyls. The intense superposed peaks between $1200-900 \mathrm{~cm}^{-1}$ correspond to different bond vibrations (stretching of C-O-C, C-O and $\mathrm{C}-\mathrm{O}-\mathrm{H}, \mathrm{O}-\mathrm{H}$ deformation and $\mathrm{C}=\mathrm{O}$ deformation).

The spectrum of pDVS is characterized by intense bands between $3000-2800$ $\mathrm{cm}^{-1}$ attributed to $\mathrm{C}-\mathrm{H}$ stretching and two intense bands in 1290 and $1110 \mathrm{~cm}^{-1}$ corresponding to $\mathrm{S}=\mathrm{O}$ asymmetric and symmetric stretching, respectively.

The spectra of the different HA-DVS polymer networks slightly differ from that of the HA film. The broad absorption band between 3500 and $3000 \mathrm{~cm}^{-1}$ becomes less intense and two separate peaks at $3690 \mathrm{~cm}^{-1}$ and $3620 \mathrm{~cm}^{-1}$ can be appreciated, attributed to stretching vibrations of free $\mathrm{O}-\mathrm{H}$. The broad peak at $1600 \mathrm{~cm}^{-1}$ is not present and the intensity of the peak at $1700 \mathrm{~cm}^{-1}$ is increased. 
The intense peak of the sulfone symmetric stretching is observed at $1080 \mathrm{~cm}^{-1}$ superposed to bands of the HA chain.

\subsubsection{Density}

The densities of the HA film and HA-DVS polymer networks were determined employing the Equation 2. 5. Moreover, the specific volume was determined from the inverse of the density. In Figure 3. 4 is plotted the density of each sample.

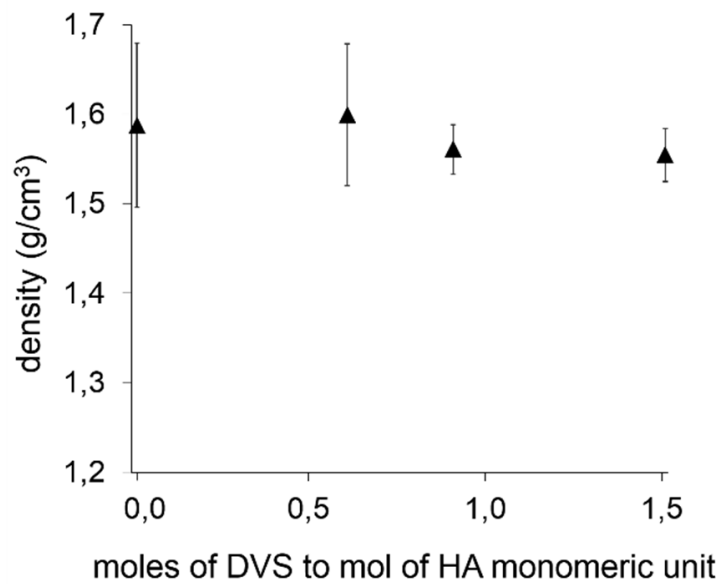

Figure 3. 4. Density of the HA film and HA-DVS polymer networks. Vertical bars show the standard deviation.

The HA film and the different HA-DVS polymer networks showed no significant differences in density, and hence this parameter was taken as the averaged values of the different samples (density of $1.575 \mathrm{~g} / \mathrm{cm}^{3}$ ).

\subsubsection{Swelling experiments}

The swelling degree, SW, of HA-DVS polymer networks immersed in different media was evaluated using Equation 2.1, taking the mass of xerogels as reference and measuring the weight at different time points until reaching the equilibrium. Thereby, the kinetics of swelling was determined and the effect of the amount of DVS on the swelling degree was evaluated. In order to achieve the influence of $\mathrm{pH}$ value and ionic strength in the $S W$, different media were employed. Media with controlled ionic strength and $\mathrm{pH}$ were prepared using a buffer system, which is able to obtain a wide range of values by altering the 
amount of each salt. In this study, a single buffer system was chosen with the aim of reducing undesirable effects on swelling due to the presence of different ions. The composition of each media and the values of ionic strength and $\mathrm{pH}$ are summarized in Table 3. 1.

Table 3. 1. Salt composition of the buffer systems.

\begin{tabular}{|c|c|c|c|c|c|}
\hline \multirow{2}{*}{$\begin{array}{l}\mathrm{pH} \text { at } \\
25 \stackrel{\circ}{\circ}\end{array}$} & \multicolumn{2}{|c|}{$\begin{array}{l}\text { Salt concentration }(\mathrm{g} / \mathrm{l}) \text { to obtain } \\
\text { buffers with different } \mathrm{pH}\end{array}$} & \multicolumn{3}{|c|}{$\begin{array}{c}\mathrm{KCl}(\mathrm{g} / \mathrm{l}) \text { to obtain different } \\
\text { ionic strength at different } \mathrm{pH} \\
\text { values }\end{array}$} \\
\hline & $\mathrm{Na}_{2} \mathrm{HPO}_{4} \cdot 12 \mathrm{H}_{2} \mathrm{O}$ & $\mathrm{H}_{3} \mathrm{C}_{6} \mathrm{H}_{5} \mathrm{O}_{7} \cdot \mathrm{H}_{2} \mathrm{O}$ & $0.5 \mathrm{M}$ & $1 \mathrm{M}$ & $2 \mathrm{M}$ \\
\hline 3 & 14.7 & 16.7 & & 68.7 & \\
\hline 7 & 58.9 & 3.7 & 5.4 & 42.7 & 117.3 \\
\hline 8 & 69.6 & 0.6 & & 32.9 & \\
\hline
\end{tabular}

After preparing each solution and after the samples reached the swelling equilibrium, the $\mathrm{pH}$ in each medium was recorded. Therefore, all the $\mathrm{pH}$ measurements resulted in values of \pm 0.3 with respect to the theoretical ones.

Additionally, from the kinetics of swelling, the type of transport that governs the diffusion process was determined. In first instance, the diffusion was supposed to be Fickian, and the kinetics of swelling was plotted using the following equation [361]:

$\frac{M_{t}}{M_{\infty}}=k \cdot \sqrt{t}$

[Equation 3.1]

where, $M_{t}$ and $M_{\infty}$ are the mass of water in the hydrogel at time $t$ and at swelling equilibrium, respectively, and $k$ is a constant defined by the slope of the line from the graph obtained from plotting the left hand of the equation as a function of the square root of time.

The following figures display the effect of media with different $\mathrm{pH}$ values $(3,7$ and 8) for a constant ionic strength of $1 \mathrm{M}$ on the swelling of the HA-DVS polymer networks. Additionally the curves derived from Equation 3.1 are included for each medium. 


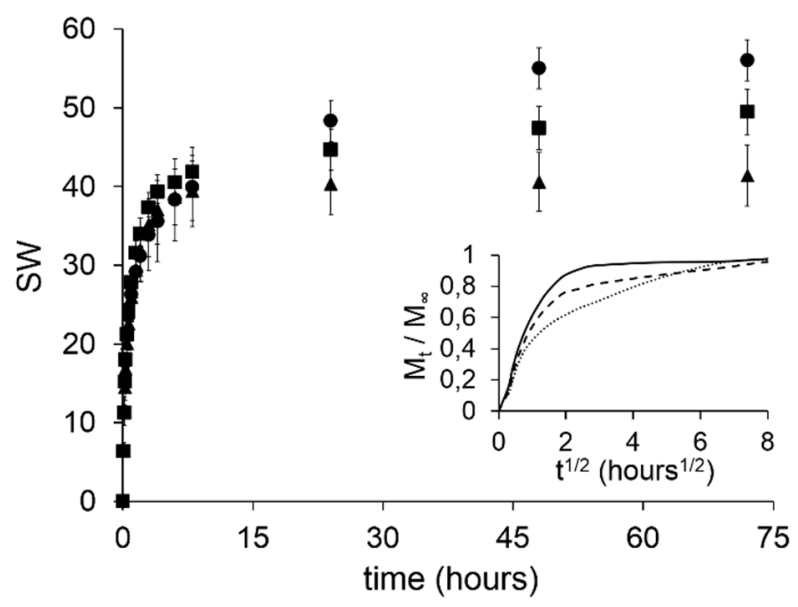

Figure 3. 5. Kinetics of swelling of solv_HA:DVS 1:0.6 samples in media with constant ionic strength $1 \mathrm{M}$ and different pH values: 3 (triangle dots), 7 (square dots) and 8 (circle dots). Vertical bars show the standard deviation of the experimental data. Inset shows the approximation of swelling to a Fickian diffusion at different pH values: 3 (solid line), 7 (dashed line) and 8 (dotted line).

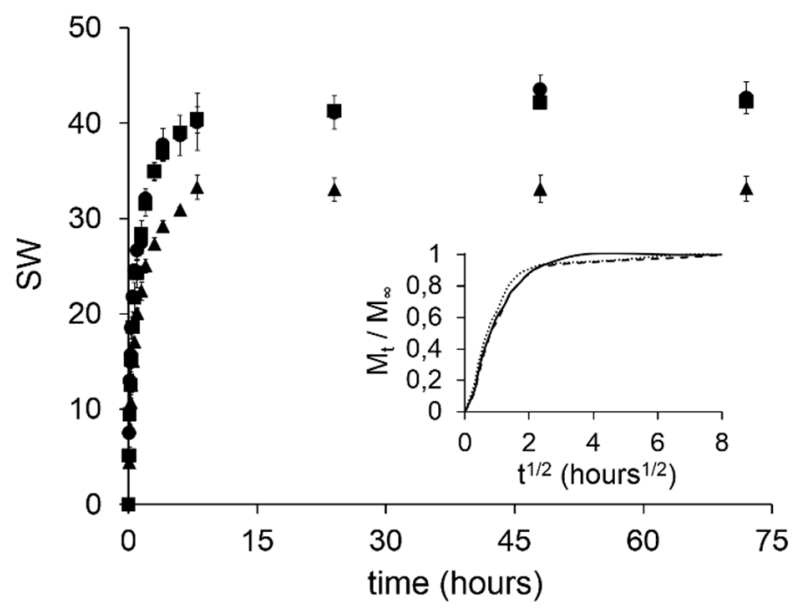

Figure 3. 6. Kinetics of swelling of solv_HA:DVS 1:0.9 samples in media with constant ionic strength $1 \mathrm{M}$ and different pH values: 3 (triangle dots), 7 (square dots) and 8 (circle dots). Vertical bars show the standard deviation of the experimental data. Inset shows the approximation of swelling to a Fickian diffusion at different $\mathrm{pH}$ values: 3 (solid line), 7 (dashed line) and 8 (dotted line). 


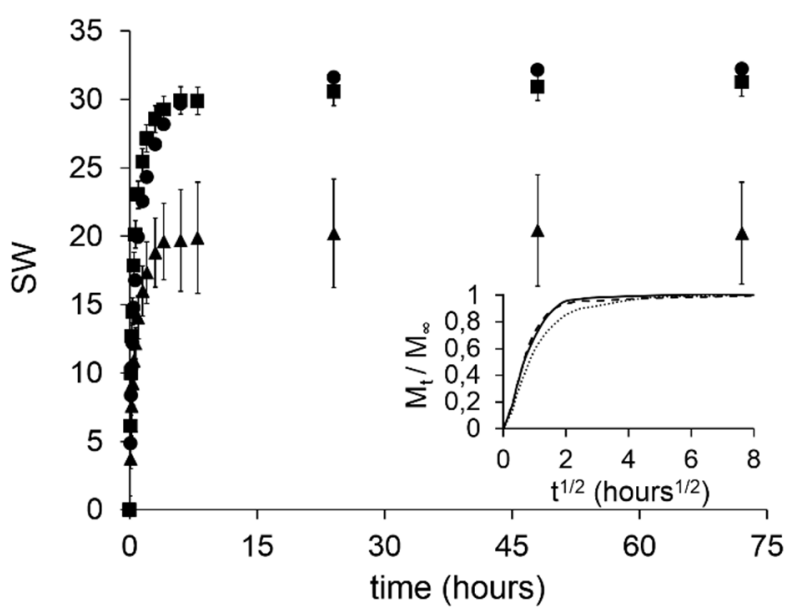

Figure 3. 7. Kinetics of swelling of solv_HA:DVS 1:1.5 samples in media with constant ionic strength $1 \mathrm{M}$ and different $\mathrm{pH}$ values: 3 (triangle dots), 7 (square dots) and 8 (circle dots). Vertical bars show the standard deviation of the experimental data. Inset shows the approximation of swelling to a Fickian diffusion at different pH values: 3 (solid line), 7 (dashed line) and 8 (dotted line).

The swelling at constant ionic strength resulted to have a similar trend (Figure 3 . 5, Figure 3. 6 and Figure 3. 7): the swelling is fast during the first 10 hours and becomes asymptotic stable. Moreover, figures evidence that the higher the HA:DVS molar ratio, the lower the swelling degree and the earlier stabilization. The HA:DVS 1:0.6 samples reach swelling degrees between 30 and 60, the HA:DVS 1:0.9 samples range from 35 to 55 and the solv_HA:DVS 1:1.5 samples reach swelling degrees between 15 and 30 . On the other hand, $\mathrm{pH}$ influences the swelling degree: the lower the $\mathrm{pH}$ value, the lower the swelling reached is. This effect is increased in HA-DVS polymer networks with lower DVS amounts. However, the swelling degree at $\mathrm{pH} 7$ and 8 show significant differences only for solv_HA:DVS 1:0.6 samples.

The figures below show the effect of media with different ionic strengths $(0.5,1$ and $2 \mathrm{M}$ ) at controlled $\mathrm{pH}$ of 7 on the swelling degree of the different HA-DVS polymer networks. 


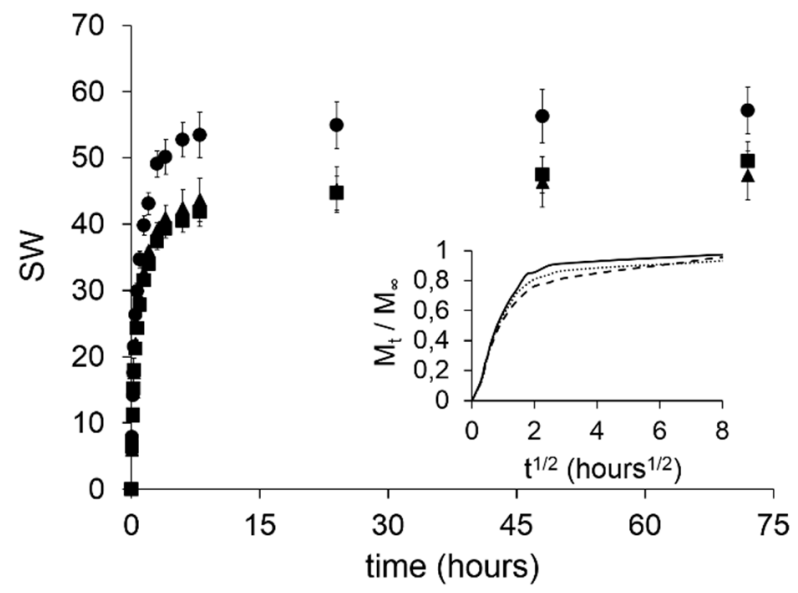

Figure 3. 8. Kinetics of swelling of solv_HA:DVS 1:0.6 samples in media at $\mathrm{pH} 7$ and different ionic strengths: $0.5 \mathrm{M}$ (circles), $1 \mathrm{M}$ (squares) and $2 \mathrm{M}$ (triangles). Vertical bars show the standard deviation of the experimental data. Inset shows the approximation of swelling to a Fickian diffusion at different ionic strengths: $0.5 \mathrm{M}$ (solid line), $1 \mathrm{M}$ (dashed line) and $2 \mathrm{M}$ (dotted line).

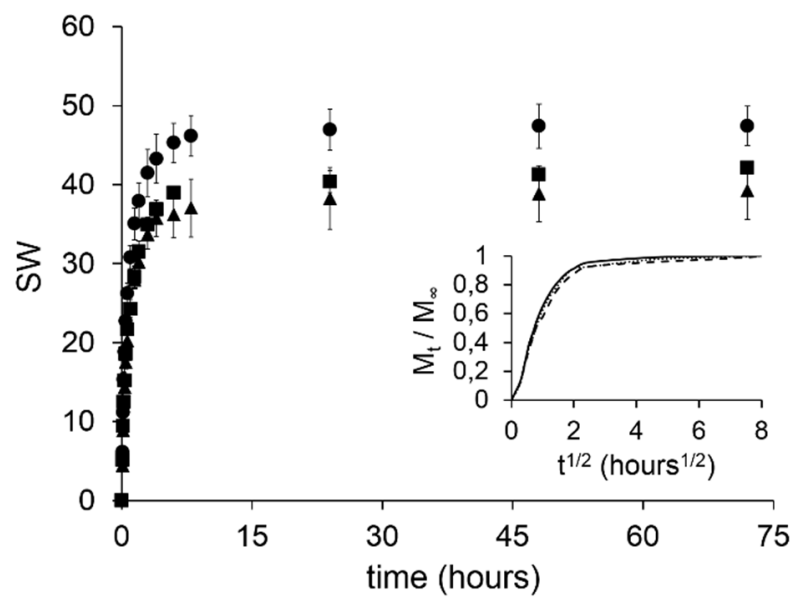

Figure 3. 9. Kinetics of swelling of solv_HA:DVS 1:0.9 samples in media at pH 7 and different ionic strengths: $0.5 \mathrm{M}$ (circles), $1 \mathrm{M}$ (squares) and $2 \mathrm{M}$ (triangles). Vertical bars show the standard deviation of the experimental data. Inset shows the approximation of swelling to a Fickian diffusion at different ionic strengths: $0.5 \mathrm{M}$ (solid line), $1 \mathrm{M}$ (dashed line) and $2 \mathrm{M}$ (dotted line). 


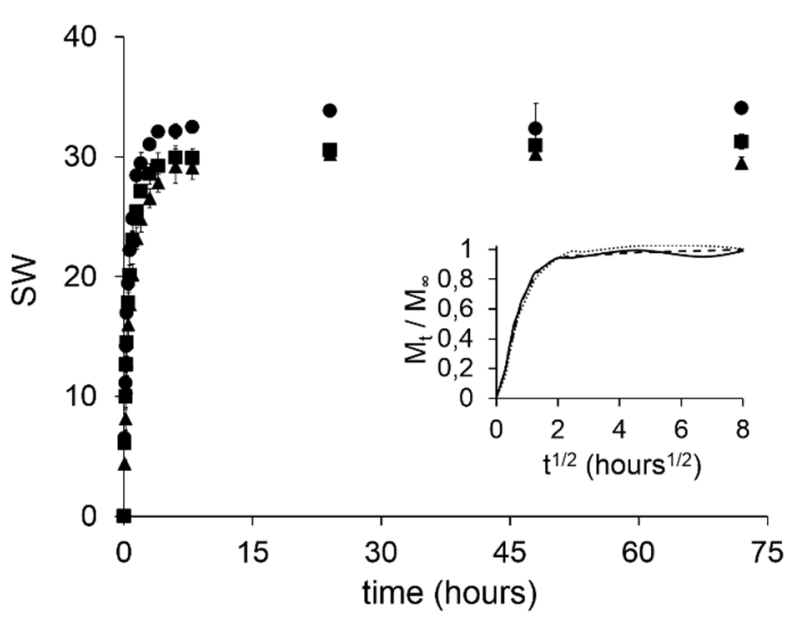

Figure 3. 10. Kinetics of swelling of solv_HA:DVS 1:0.1.5 samples in media at pH 7 and different ionic strengths: $0.5 \mathrm{M}$ (circles), $1 \mathrm{M}$ (squares) and $2 \mathrm{M}$ (triangles). Vertical bars show the standard deviation of the experimental data. Inset shows the approximation of swelling to a Fickian diffusion at different ionic strengths: $0.5 \mathrm{M}$ (solid line), $1 \mathrm{M}$ (dashed line) and $2 \mathrm{M}$ (dotted line).

The variation of ionic strength and $\mathrm{pH}$ gave similar trends on the swelling kinetics of the different HA-DVS polymer networks (Figure 3. 8, Figure 3. 9 and Figure 3. $10)$. In addition, as the case of different $\mathrm{pH}$ values, the higher molar ratio of DVS to HA the lower the swelling degree and the earlier stabilization. At different ionic strength, the swelling degree in solv_HA:DVS 1:0.6 samples reaches values between 30 and 40, in the solv_HA:DVS 1:0.9 samples from 30 to 50 and in the solv_HA:DVS 1:1.5 samples reaches values around 30. On the other hand, the lower the ionic strength the higher the swelling degree, this effect is more noticeable in the HA-DVS polymer networks with lower DVS amounts. Moreover, the different samples showed no significant differences in their swelling degree when the media with ionic strength of $1 \mathrm{M}$ and $2 \mathrm{M}$ are compared.

In order to determine the swelling behaviour of the HA-DVS polymer networks in media mimicking the physiological one, the kinetics of swelling in the different HA-DVS polymer networks immersed in $0.15 \mathrm{M}$ PBS was determined. The salt content of $0.15 \mathrm{M}$ PBS is summarized in Table 3. 2. 
Table 3. 2. Salt composition of $0.15 \mathrm{M}$ PBS.

\begin{tabular}{cc}
\hline Salt specie & Concentration (g/L) \\
\hline $\mathrm{NaCl}$ & 8.0 \\
$\mathrm{KCl}$ & 0.2 \\
$\mathrm{Na}_{2} \mathrm{HPO}_{4}$ & 1.4 \\
$\mathrm{KH}_{2} \mathrm{PO}_{4}$ & 0.2 \\
\hline
\end{tabular}

The swelling kinetics of the different HA-DVS polymer networks in $0.15 \mathrm{M}$ PBS is plotted below:

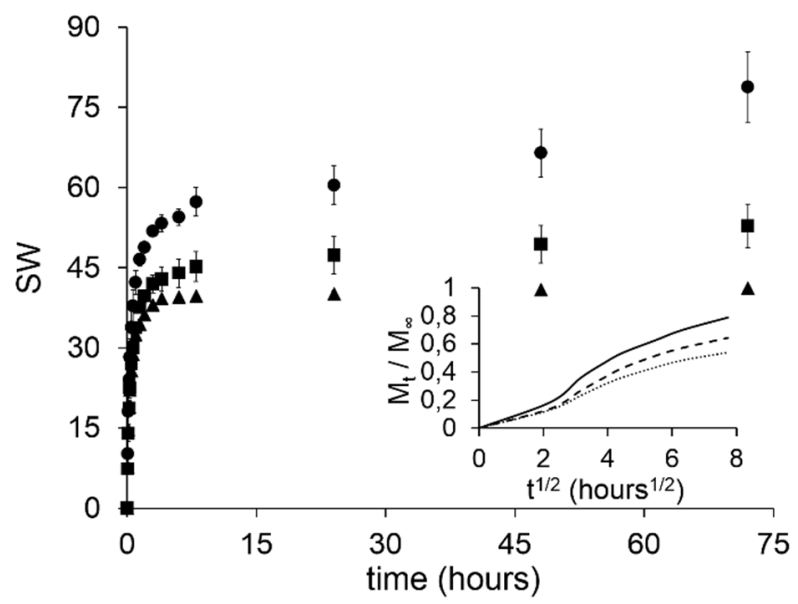

Figure 3. 11. Kinetics of swelling in $0.15 \mathrm{M}$ PBS of the different HA-DVS polymer networks: solv_HA:DVS 1:0.6 (circles), solv_HA:DVS 1:0.9 (squares) and solv_HA:DVS 1:1.5 (triangles). Vertical bars represent standard deviation of experimental data.

The kinetics of swelling of the HA-DVS polymer networks in 0.15 M PBS arises a similar trend than those of samples immersed in other media: the swelling was fast during the first 10 hours and became asymptotic stable for longer times. In general, the swelling degree of the different samples immersed in $0.15 \mathrm{M}$ PBS resulted to be higher than that in other media. Moreover, the HA-DVS polymer networks with higher HA:DVS molar ratio show a lower swelling degree. 
In addition, the equilibrium water content, EWC, of the samples, considered the swelling degree after 72 hours in immersion, was determined for each composition and medium. In this study, the EWC of samples immersed in deionized water was included.
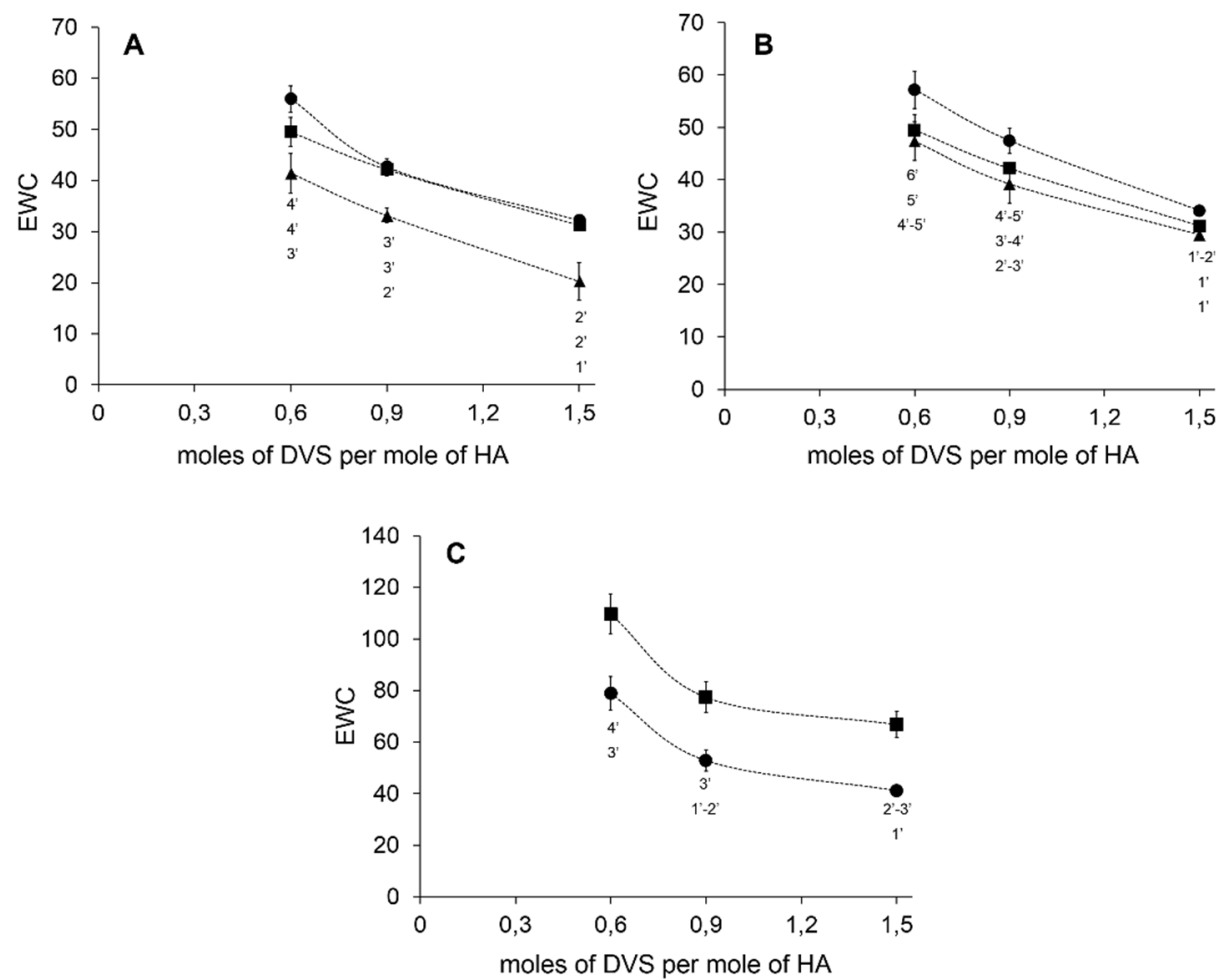

Figure 3. 12. Representation of $E W C$ as function of the molar ratio of DVS to HA monomeric unit in the different swollen media. (A) lonic strength at $1 \mathrm{M}$ and different $\mathrm{pH}$ values: 8 (circles), 7 (squares) and 3 (triangles). (B) Controlled pH 7 and variable ionic strength: $0.5 \mathrm{M}$ (circles), $1 \mathrm{M}$ (squares) and

$2 \mathrm{M}$ (triangles). (C) PBS (circle dots) and water (square dots). Vertical bars show the standard deviation of the experimental data.

Figure 3. 12 shows that the amount of DVS added in the crosslinking reaction affect the EWC value of the samples in all the media; the higher the amount of DVS the lower the EWC value. This effect turns more significant comparing solv_HA:DVS 1:1.5 with each other compositions. A higher ionic strength reduces $E W C$ values, but this reduction in EWC seems to become stable for ionic strengths up $1 \mathrm{M}$ (Figure 3.12 A). Additionally, the EWC value of the HA-DVS polymer networks with larger amounts of DVS is less affected by the ionic strength. Contrarily, the higher $\mathrm{pH}$ in medium increases the EWC values, 
observing no significant differences between $\mathrm{pH} 7$ and 8 (Figure 3. 12 B). Moreover, the difference in the EWC values between each HA-DVS composition remains constant for the different media with variable $\mathrm{pH}$. The swelling of the HADVS polymer networks in $0.15 \mathrm{M}$ PBS and water displayed higher values than those in media with controlled pH and ionic strength (Figure 3. $12 \mathrm{C}$ ). The EWC value in water is significant higher than in $0.15 \mathrm{M}$ PBS and this difference remains approximately constant for all the compositions. However, the EWC value in both media is stabilized with the amount of DVS.

On the other hand, the insets in Figure 3. 5 to Figure 3. 11 show curves with similar trends for the different HA-DVS polymer networks immersed in the different media. At earlier swelling times, the curves show two straight regions: the first one at initial times with a lower slope and the second one with a slight increment of the slope. In general, these linear regions range between swelling ratios of 0 and 0.6 , after this point, the curves are asymptotically stabilized until the value of 1 .

In a diffusion process governed by a Fickian transport, the swelling depends on the square root of time. Therefore, the curves obtained from Equation 3.1 suggested almost two possible Fickian diffusion transport: the two earlier linear regions. In the subsequent study, the first linear region was discarded because of the swollen hydrogel at the initial stages showed a transition from a glass state to a rubbery-like one. In order to corroborate the Fickian transport in the swollen hydrogels, the power law equation was employed:

$\frac{M_{t}}{M_{0}}=K \cdot t^{n}$

[Equation 3. 2]

where $M_{t}$ is the amount of water in the swollen hydrogel at time $t, M_{0}$ the mass of the xerogel, $K$ is the swelling constant, and $n$ is the swelling exponent.

Therefore, from the logarithmic representation of Equation 3. 2 for $M_{t} / M_{\infty}$ values between 0.2 and 0.6 was determined the swelling exponent, $n$, which value gives the dependence of swelling with time, and hence the type of transport process. The $n$ values for the different samples immersed in the different media are shown in the following table: 
Table 3. 3. Values of the swelling power exponent, $n$, of the HA-DVS polymer networks in the different swollen media.

\begin{tabular}{cccc}
\hline & \multicolumn{3}{c}{ swelling power exponent } \\
\cline { 2 - 4 } swelling medium & solv_HA:DVS 1:0.6 & solv_HA:DVS 1:0.9 & solv_HA:DVS 1:1.5 \\
\hline $\mathrm{pH} \mathrm{7} \mathrm{and} \mathrm{I=0.5} \mathrm{M}$ & 0.48 & 0.53 & 0.52 \\
$\mathrm{pH} \mathrm{7} \mathrm{and} \mathrm{I=1} \mathrm{M}$ & 0.50 & 0.53 & 0.57 \\
$\mathrm{pH} \mathrm{7} \mathrm{and} \mathrm{I=2M}$ & 0.58 & 0.59 & 0.72 \\
$\mathrm{pH} 3$ and I=1 M & 0.45 & 0.54 & 0.50 \\
$\mathrm{pH} 8$ and I=1 M & 0.30 & 0.47 & 0.50 \\
0.15 M PBS & 0.47 & 0.55 & 0.44 \\
\hline
\end{tabular}

Table 3. 3 shows that diffusion in the swelling of the HA-DVS polymer networks is governed, in general, by a Fickian transport (values of $n$ around 0.5). In such case of transport, the diffusion coefficient, $D$, may be determined, fitting the swelling ratio, $M_{t} / M_{\infty}$, between 0.2 and 0.6 as function of the square root of time to a straight line:

$\frac{M_{t}}{M_{\infty}}=4 \sqrt{\frac{D}{\left.\pi \cdot\right|^{2}}} \cdot \sqrt{t}$

[Equation 3. 3]

where $l$ is the thickness of the hydrogel approximating the swelling phenomena as a diffusion process in a thin film. The thickness of the hydrogel was taken as the averaged value in the region of $M_{t} / M_{\infty}$ between 0.2 and 0.6 . Therefore, the values of the diffusion coefficient, $D$, in case of Fickian transport were collected in the following table: 
Table 3. 4. Diffusion coefficient in the HA-DVS polymer networks in the different swollen media.

\begin{tabular}{cccc}
\hline \multirow{2}{*}{ Swelling media } & \multicolumn{3}{c}{ Diffusion coefficient $\left(\mathbf{c m}^{2} / \mathbf{s} \times \mathbf{1 0}^{\mathbf{7}}\right)$} \\
\cline { 2 - 4 } & solv_HA:DVS 1:0.6 & solv_HA:DVS 1:0.9 & solv_HA:DVS 1:1.5 \\
\hline $\mathrm{pH} \mathrm{7} \mathrm{and} \mathrm{I=0.5} \mathrm{M}$ & 3.92 & 4.23 & 5.59 \\
$\mathrm{pH} \mathrm{7} \mathrm{and} \mathrm{I=1} \mathrm{M}$ & 2.81 & 2.85 & 5.17 \\
$\mathrm{pH} \mathrm{7}$ and I=2 M & 0.46 & 2.91 & --- \\
$\mathrm{pH} \mathrm{3}$ and I=1 M & 2.62 & 2.72 & 3.19 \\
$\mathrm{pH} 8$ and I=1 M & --- & 4.25 & 1.47 \\
0.15 M PBS & 3.79 & 4.76 & 6.88 \\
\hline
\end{tabular}

\subsubsection{Volumetric swelling}

In order to corroborate the isotropy of swelling, the volumetric swelling in equilibrium determined from geometrical measures (Qisotropic) and from gravimetric determination $(Q)$ were calculated (see Equation 2. 3 and Equation 2. 4, respectively). The hypothesis that in isotropic swelling the HA-DVS polymer networks are deformed with similar ratio in all their directions arises that $Q$ and Qisotropic should have similar values. The volumetric swelling determined by both methods in the different media was evaluated for all the HA-DVS polymer networks compositions.

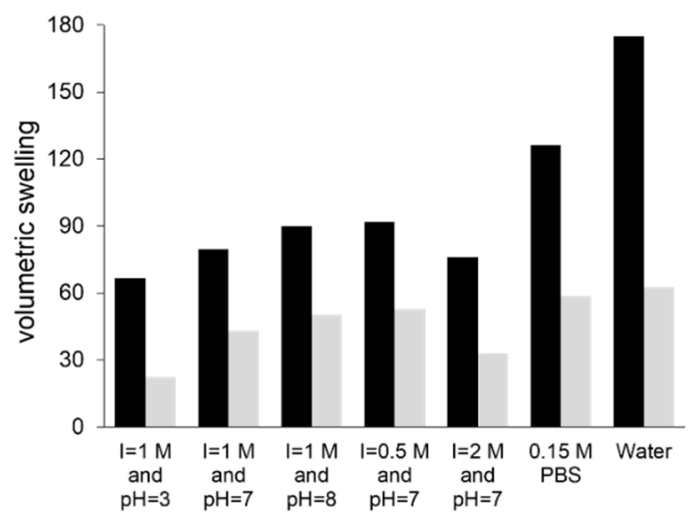

Figure 3. 13. Volumetric swelling of solv_HA:DVS 1:0.6 samples immersed in different media calculated gravimetrically, $\mathrm{Q}$ (black bars), and geometrically, $\mathrm{Q}_{\text {isotropic }}$ (grey bars). 


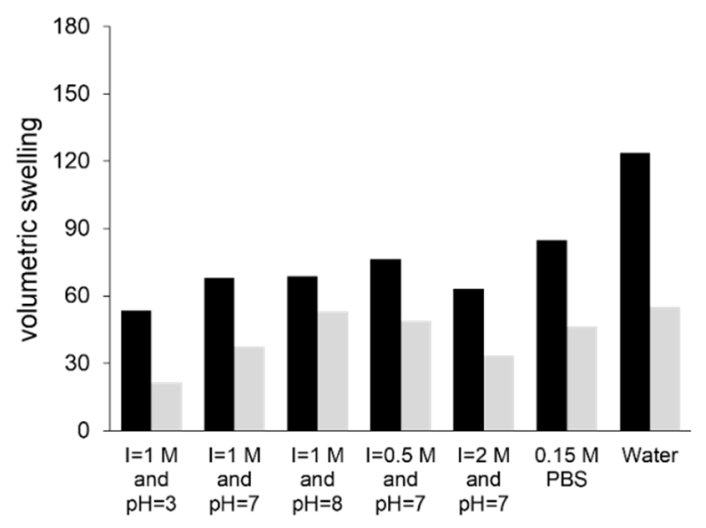

Figure 3. 14. Volumetric swelling of solv_HA:DVS 1:0.9 samples immersed in different media calculated gravimetrically, $\mathrm{Q}$ (black bars), and geometrically, $\mathrm{Q}_{\text {isotropic }}$ (grey bars).

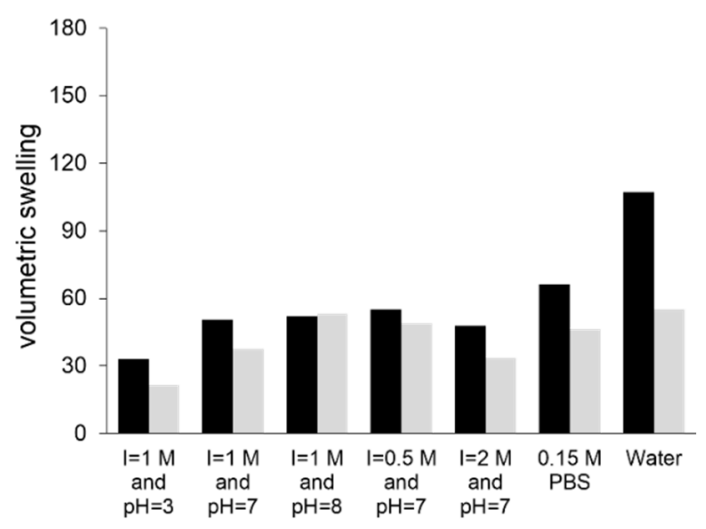

Figure 3. 15. Volumetric swelling of solv_HA:DVS 1:1.5 samples immersed in different media calculated gravimetrically, Q (black bars), and geometrically, $Q_{\text {isotropic }}$ (grey bars).

Figures 3.13 to 3.15 show that the volumetric swelling is proportional to the EWC values. Therefore, the volumetric swelling shows a similar behaviour of $E W C$ for each medium, hence increasing ionic strength, and reducing $\mathrm{pH}$ and the DVS amount lower volumetric swelling values were obtained. The values of $Q$ and Qisotropic arise, in general, an anisotropic swelling. Differences between both parameters are increased in media where the swelling degree is higher. 


\subsubsection{Determination of the degree of crosslinking}

The degree of crosslinking of the different HA-DVS polymer networks was determined by indirect and direct methods.

The indirect method includes the Flory theory for polymer-solvent systems $[362,363]$. The results derived from the equilibrium water content were employed to evaluate the degree of crosslinking. This method is an approximation since it requires an isotropic swelling of the hydrogels and not take into account some considerations of $\mathrm{HA}$ molecules including chains interactions and entanglements. The Flory theory for polymeric networks at equilibrium swelling determines that the crosslinking density, $\rho_{X}$, may be calculated setting zero the following expression:

$\ln \left(1-\Phi_{2}\right)+\Phi_{2}+X \Phi_{2}{ }^{2}+\rho_{x} V_{1} \Phi_{0}\left(\left(\frac{\Phi_{2}}{\Phi_{0}}\right)^{\frac{1}{3}}-0.5 \frac{\Phi_{2}}{\Phi_{0}}\right)+V_{1}\left[2 C_{S}^{*}-\sqrt{\left(\frac{i \Phi_{2}}{V_{U}}\right)^{2}+4\left(C_{S}{ }^{*}\right)^{2}}\right.$

[Equation 3. 4]

where, $\Phi_{2}$ and $\Phi_{0}$ are the polymer fractions in the hydrogel at equilibrium swelling and after the crosslinking reaction, respectively. $V_{1}$ is the molar volume of water $\left(18 \mathrm{~cm}^{3} / \mathrm{mol}\right) \cdot X$ is the Flory polymer-solvent interaction parameter (assumed to be 0.473 for low fractions of $\mathrm{HA}$ in water and considering that $\mathrm{HA}$ and Dextran have a similar behaviour [213,364]). $C_{S}{ }^{*}$ is the salt concentration outside the hydrogel (assumed zero for pure water). $i$ is the degree of ionization of HA (assumed 1 for neutral $\mathrm{pH}$ ). $V_{U}$ is the molar volume of ionisable groups per $\mathrm{HA}$ disaccharide unit $\left(250 \mathrm{~cm}^{3} / \mathrm{mol}\right)$.

Additionally, $\Phi_{2}$ and $\Phi_{0}$ may be calculated from the degree of mass swelling, $Q_{M}$, at equilibrium swelling and after the crosslinking reaction, respectively, by the following expression:

$\Phi^{-1}=1+\frac{\rho_{p}}{\rho_{s}}\left(Q_{M^{-1}}\right)$

[Equation 3.5]

where $\rho_{p}$ and $\rho_{s}$ are the densities of the xerogel $\left(1.575 \mathrm{~g} / \mathrm{cm}^{3}\right.$ for $\mathrm{HA}$, calculated in section 3.1.2.2) and solvent $\left(1 \mathrm{~g} / \mathrm{cm}^{3}\right.$ for water), respectively. 
The molecular weight between crosslinks, $\bar{M}_{c}$, in the different HA-DVS polymer networks may be determined from $\rho_{x}$ as follows:

$\bar{M}_{c}=\frac{1}{\rho_{x} \cdot \bar{v}}$

[Equation 3.6]

where, $\bar{v}$ is the specific volume of $\mathrm{HA}\left(0.635 \mathrm{~cm}^{3} / \mathrm{g}\right.$, the inverse of the density calculated in section 3.1.2.2).

Additionally, the number of disaccharide units between crosslinks, $\overline{\mathrm{d}}$, may be determined from $\bar{M}_{c}$ taking into account the molecular weight of the HA monomeric unit $(400 \mathrm{~g} / \mathrm{mol})$. The different parameters derived from the swelling experiments in water are collected in Table 3. 5.

Table 3. 5. Parameters determined from the Flory theory for polymer-solvent systems.

\begin{tabular}{cccc}
\hline Composition & solv_HA:DVS & solv_HA:DVS & solv_HA:DVS \\
& $\mathbf{1}: \mathbf{0 . 6}$ & $\mathbf{1}: \mathbf{0 . 9}$ & \\
\hline $\mathrm{QM}_{\mathrm{M}}$ & 97.4 & 74.5 & 61.1 \\
$\rho_{\mathrm{x}}\left(\mathrm{mol} / \mathrm{cm}^{3}\right)$ & $5.8 \times 10^{-4}$ & $7.0 \times 10^{-4}$ & $8.2 \times 10^{-4}$ \\
$\overline{\mathrm{M}}_{\mathrm{c}}(\mathrm{kDa})$ & 2.7 & 2.3 & 1.9 \\
$\overline{\mathrm{d}}$ & 6.9 & 5.6 & 4.8 \\
$\begin{array}{c}\text { degree of } \\
\text { crosslinking }(\%)\end{array}$ & 14.6 & 17.7 & 20.7 \\
\hline
\end{tabular}

In order to determine the DVS incorporated in the HA-DVS polymer networks, the atomic weight fraction of sulphur was determined by elemental analysis CHNS (see section 2.3). Taking into account the stoichiometry of the crosslinked hyaluronan (see Figure 1.6) and the atomic mass of each element, the degree of crosslinking may be determined, and hence the values of $\bar{M}_{c}$ and $\bar{d}$ of the different compositions. The atomic content of each element in the samples is collected in Table 3. 6. 
Table 3. 6. Atomic weight fraction of nitrogen, carbon, hydrogen and sulphur.

\begin{tabular}{ccccc}
\hline \multirow{2}{*}{ Sample } & \multicolumn{4}{c}{ Atomic weight fraction (in percentage) } \\
\cline { 2 - 5 } & Nitrogen & Carbon & Hydrogen & Sulphur \\
\hline native HA & 3.2 & 32.7 & 9.1 & \\
solv_HA:DVS 1:0.6 & 2.9 & 31.8 & 8.4 & 0.9 \\
solv_HA:DVS 1:0.9 & 2.7 & 31.9 & 8.1 & 1.5 \\
solv_HA:DVS 1:1.5 & 2.6 & 32.3 & 8.7 & 2.4 \\
\hline
\end{tabular}

The validity of the method on quantifying the atomic content was achieved comparing the relation between the atomic weight of carbon and nitrogen determined in the native HA with the stoichiometric ratio of both elements. The values of the different parameters of the crosslinking (degree of crosslinking, $\bar{M}_{c}$ and $\bar{d}$ ) were determined relating the sulphur with both carbon and nitrogen contents and taking into account the stoichiometry between them (Table 3. 7).

Table 3. 7. Parameters of the crosslinking of HA with DVS determined by elemental analysis CHNS.

\begin{tabular}{cccc}
\hline Composition & Solv_HA:DVS & Solv_HA:DVS & solv_HA:DVS \\
& & $\mathbf{1}: \mathbf{0 . 6}$ & $\mathbf{1}: \mathbf{1 . 5}$ \\
\hline $\bar{M}_{\mathrm{c}}(\mathrm{Da})$ & 1219.2 & 752.6 & 459.7 \\
$\overline{\mathrm{d}}$ & 3.0 & 1.9 & 1.1 \\
degree of crosslinking (\%) & 32.9 & 53.3 & 87.2 \\
\hline
\end{tabular}

\subsubsection{Compression tests}

In order to determine the influence of the degree of crosslinking in the mechanical properties of the HA-DVS polymer networks, compression tests were performed. The different HA-DVS polymer networks at equilibrium swelling were subjected to unconfined compression under immersion in the corresponding medium. Moreover, the initial modulus to evaluate the elasticity of the materials was determined from the stress-strain curves (Equation 2.9). An initial modulus was calculated from the initial slope of the stress-strain curve, until a unitary strain of 0.1 .

Stress-strain curves of the different HA-DVS polymer networks compositions immersed in media with controlled ionic strength and different $\mathrm{pH}$ values were determined. 

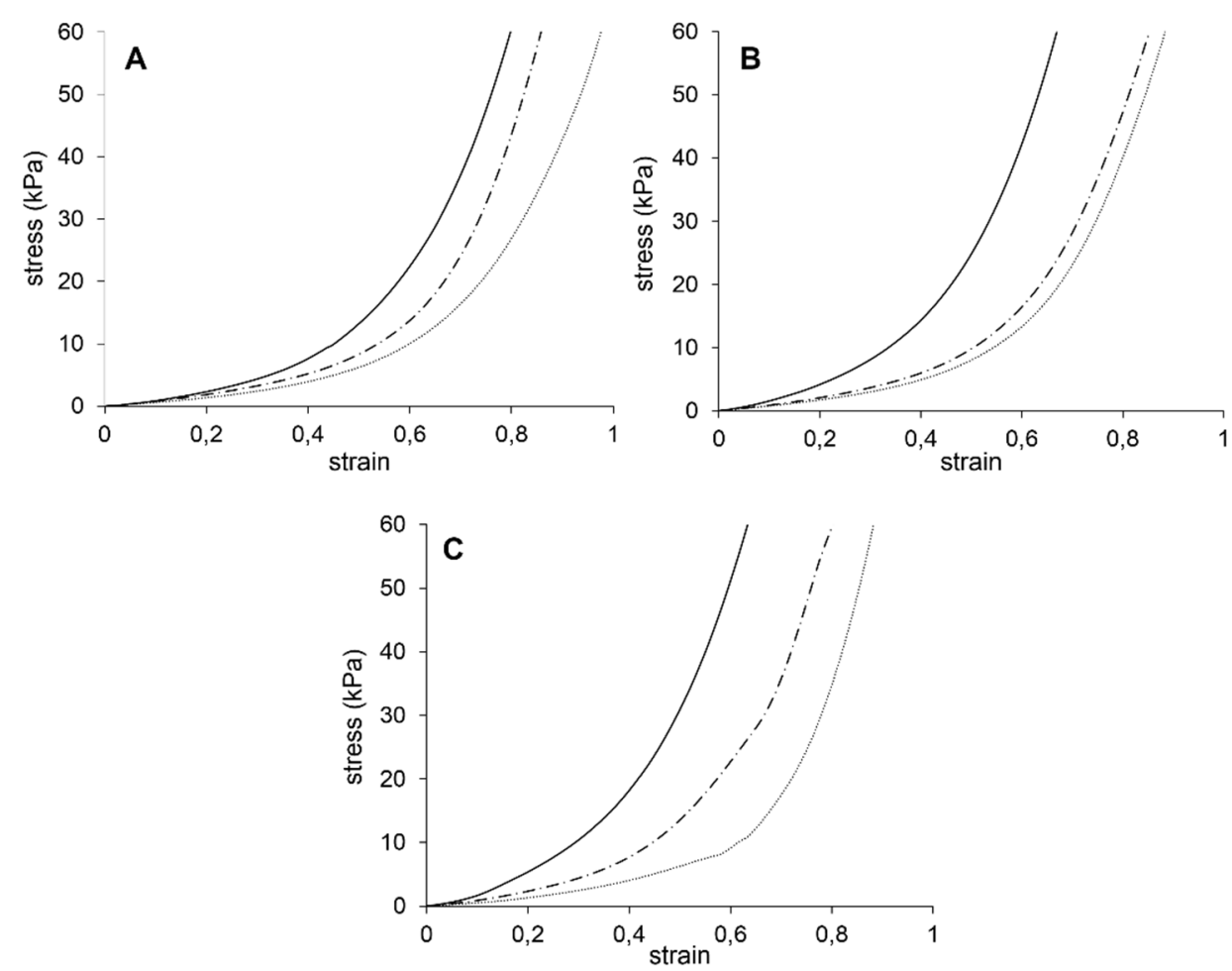

Figure 3. 16. Stress-strain curves of the HA-DVS polymer networks: A) solv_HA:DVS_1:0.6 samples, B) solv_HA:DVS_1:0.9 samples and C) solv_HA:DVS_1:1.5 samples. Each composition was measured in media with constant ionic strength of $1 \mathrm{M}$ and different $\mathrm{pH}$ values: 3 (solid line), 7 (dashed line) and 8 (dotted line).

For the media with different $\mathrm{pH}$ values, the stress-strain curves (Figure 3. 16) show a similar trend: an initial and large elastic region, which turns to a vertical asymptotic stabilization of the strain at advanced compression. During the compression tests, samples reached large unitary strains, between 0.5 and 1 , without observing the breaking stress in the whole range studied. Moreover, after the test, the samples show a slightly deswelling but practically recover their initial dimensions after a short time. However, the HA-DVS polymer networks show slightly differences depending on their composition. In general, higher amounts of DVS lead to a lower elastic region and the stabilization of the strain occurs earlier. In addition, the $\mathrm{pH}$ of the media affects the compressibility of the samples: the higher the value of $\mathrm{pH}$, the larger the elastic region and the reached strains.

In order to study the effect of ionic strength on mechanical properties, the HADVS polymer networks immersed in media with different ionic strengths at constant $\mathrm{pH}$ value were conducted under compression. 

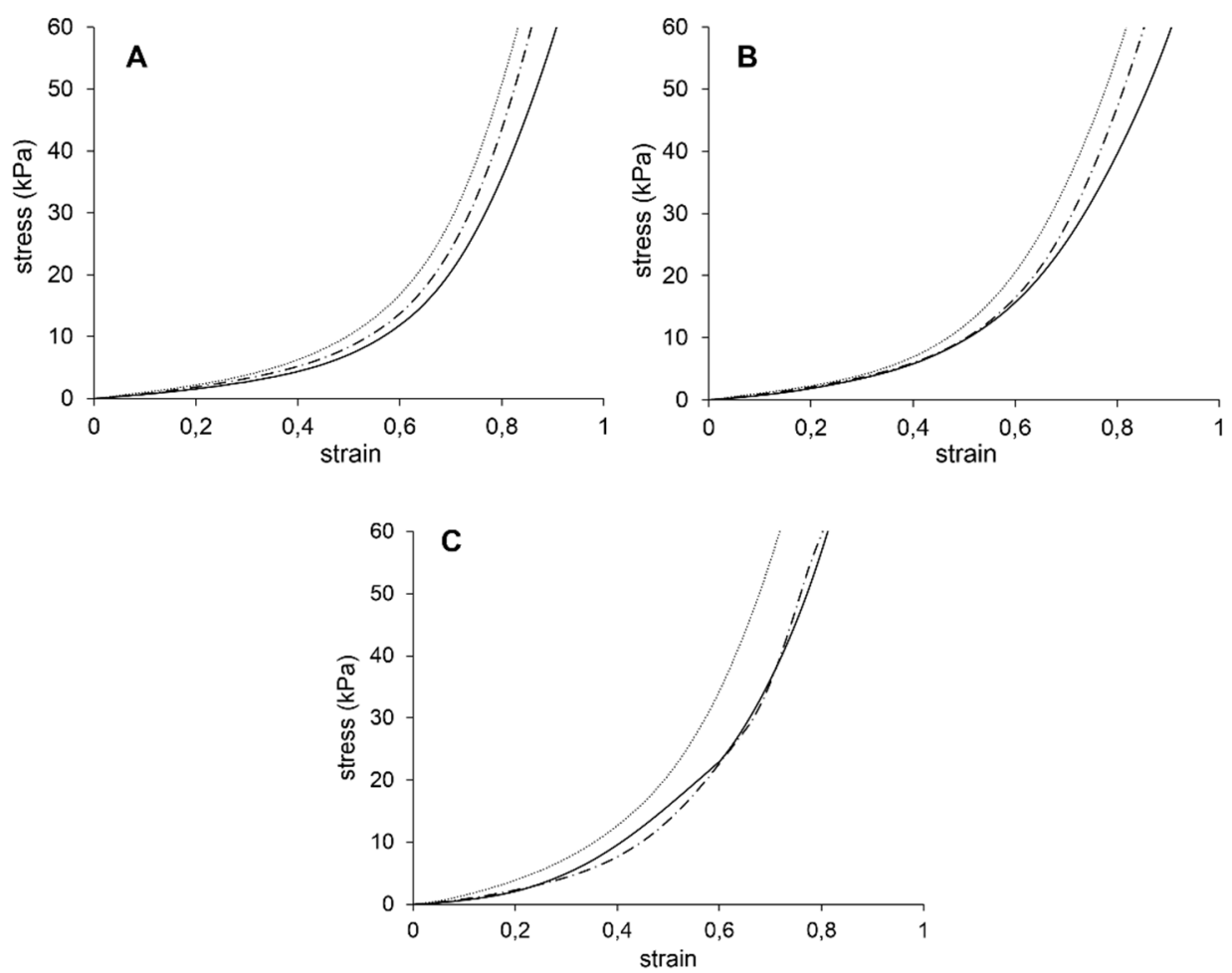

Figure 3. 17. Stress-strain curves of the HA-DVS polymer networks: A) solv_HA:DVS_1:0.6 samples, B) solv_HA:DVS_1:0.9 samples and C) solv_HA:DVS_1:1.5 samples. Each composition was measured in media at $\mathrm{pH}$ value 7 and different ionic strengths: $0.5 \mathrm{M}$ (solid line), $1 \mathrm{M}$ (dashed line) and $2 \mathrm{M}$ (dotted line).

The stress-strain curves of the HA-DVS polymer networks swollen in media with several ionic strengths (Figure 3. 17) show similar trends than in media with different $\mathrm{pH}$ (Figure 3. 16). In addition, the increment of crosslinking agent reduces the elastic region and the strains reached at advanced compression. However, in this case, the effect of ionic strength is lower than the variation on the $\mathrm{pH}$, and each HA-DVS composition shows similar mechanical properties under compression.

In order to mimic the behaviour of samples in physiological conditions, HA-DVS polymer networks were compressed immersed and in equilibrium of $0.15 \mathrm{M}$ PBS. 


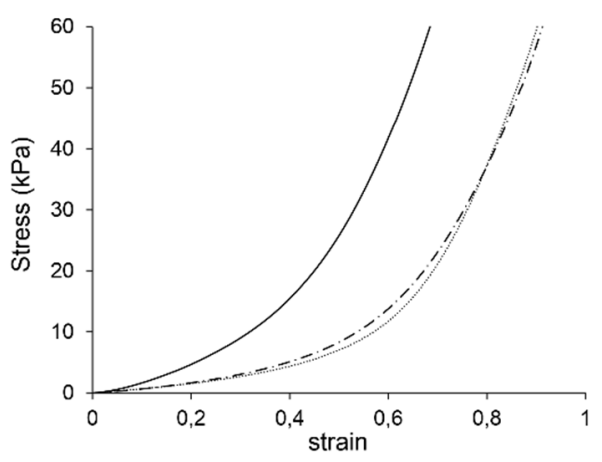

Figure 3. 18. Stress-strain curves of HA-DVS polymer networks in equilibrium swelling of $0.15 \mathrm{M}$ PBS: A) solv_HA:DVS 1:0.6 samples, B) solv_HA:DVS 1:0.9 samples and C) solv_HA:DVS 1:1.5 samples.

The HA-DVS polymer networks immersed in $0.15 \mathrm{M}$ PBS and subjected to compression reached high strains (up 0.6) (Figure 3. 18). Moreover, the stressstrain curves highlight slight discrepancies in the mechanical behaviour of solv_HA:DVS 1:1.5 samples with respect to each other compositions, which show softer mechanical properties.

The stress-strain curves show, in general, non-linear trends due to the change in the cross-section during the compression. However, this effect was neglected for short compression times, and hence an initial modulus was determined until strains of 0.1 . In order to compare the elastic properties of the HA-DVS polymer networks immersed in the different media, the initial modulus was calculated and fitted. 

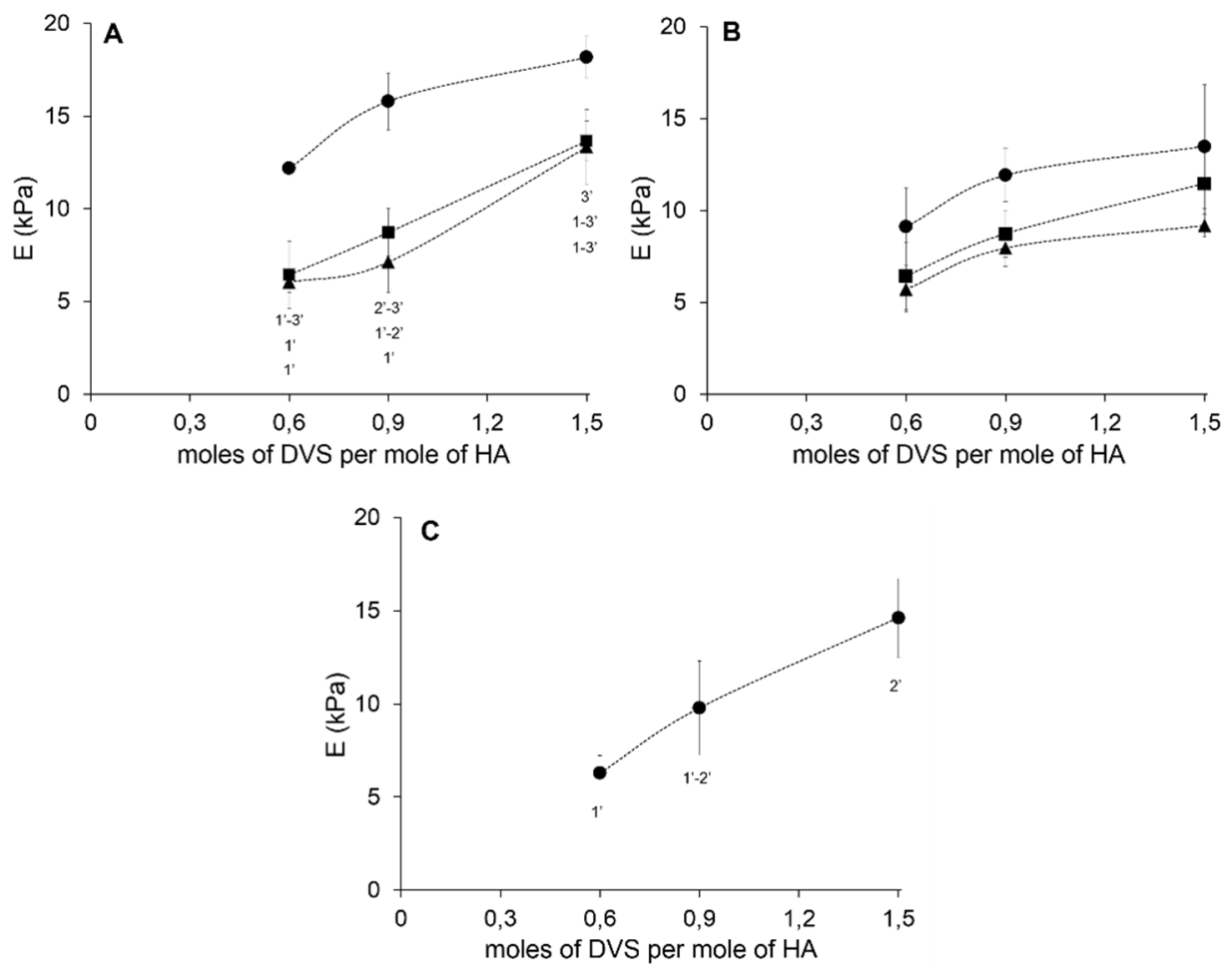

Figure 3. 19. Representation of the initial modulus as a function of the molar ratio of DVS to HA monomeric unit in samples immersed in different media. A) Media with ionic strength of $1 \mathrm{M}$ and at different $\mathrm{pH}$ : 3 (circles), 7 (squares) and 8 (triangles). B) Media with constant $\mathrm{pH}$ of 7 and different ionic strength (2 $\mathrm{M}$ (circles), $1 \mathrm{M}$ (squares) and $0.5 \mathrm{M}$ (triangles). C) $0.15 \mathrm{M}$ PBS. Vertical bars show the standard deviation of the experimental data.

The samples swollen in different media show an initial modulus, $E$, comprised between 5 and $20 \mathrm{kPa}$. The increment of crosslinking agent led to slightly higher values of $E$ for all the media studied. These discrepancies are less important between media at $\mathrm{pH} 7$ and different ionic strengths (Figure 3. $19 \mathrm{~B}$ ), but the differences increase with the variation of $\mathrm{pH}$ at ionic strength of $1 \mathrm{M}$ (Figure 3.19 A) and in $0.15 \mathrm{M}$ PBS (Figure 3. $19 \mathrm{C}$ ). In the case of media containing different ionic strengths, the values of $E$ for the different samples and media are in the range of 5 and $10 \mathrm{kPa}$, showing no significant differences. The $E$ values of the different HA-DVS polymer networks show not differences in media with controlled ionic strength $(1 \mathrm{M})$ at $\mathrm{pH} 7$ and 8 , but this value is significantly increased at $\mathrm{pH}$ 3 for all the compositions. 


\subsubsection{Thermogravimetric analysis}

In order to study the thermal stability, the HA-DVS polymer networks were conducted on thermogravimetry equipment from room temperature to $700 \stackrel{\circ}{ } \mathrm{C}$. With the aim to study the effect of the amount of DVS, thermograms of HA-DVS polymer networks were compared with those of native HA and pDVS. From the weights recorded at each temperature were determined the thermograms of remaining weight (\%) and the derivative weight loss (\%/ $\stackrel{\circ}{ } \mathrm{C})$.

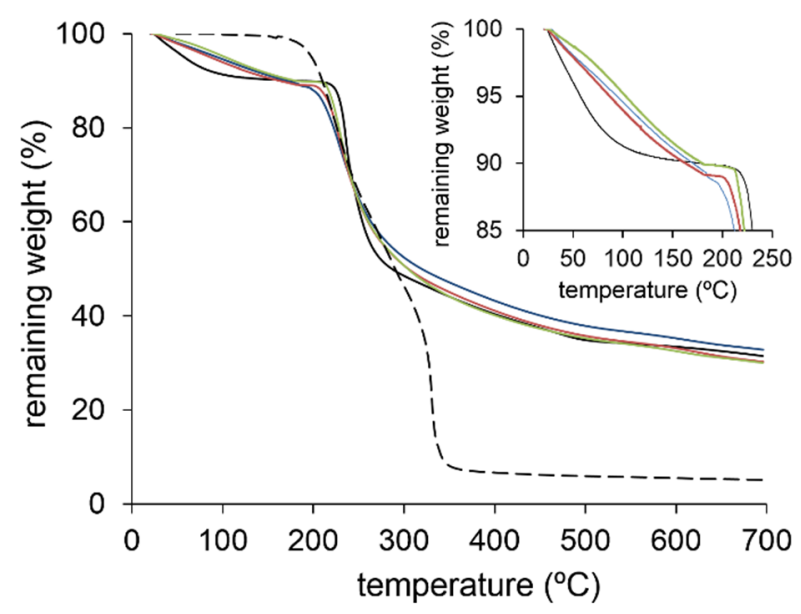

Figure 3. 20. Remaining weight thermograms obtained for native HA (black solid line), pDVS (black dotted line) and the different HA-DVS polymer networks: solv_HA:DVS_1:0.6 (blue line),

solv_HA:DVS_1:0.9 (red line) and solv_HA:DVS_1:1.5 (green line). The inset shows a detail of the thermograms of native $\mathrm{HA}$ and the different $\mathrm{HA}-\mathrm{DVS}$ polymer networks at low temperatures. 


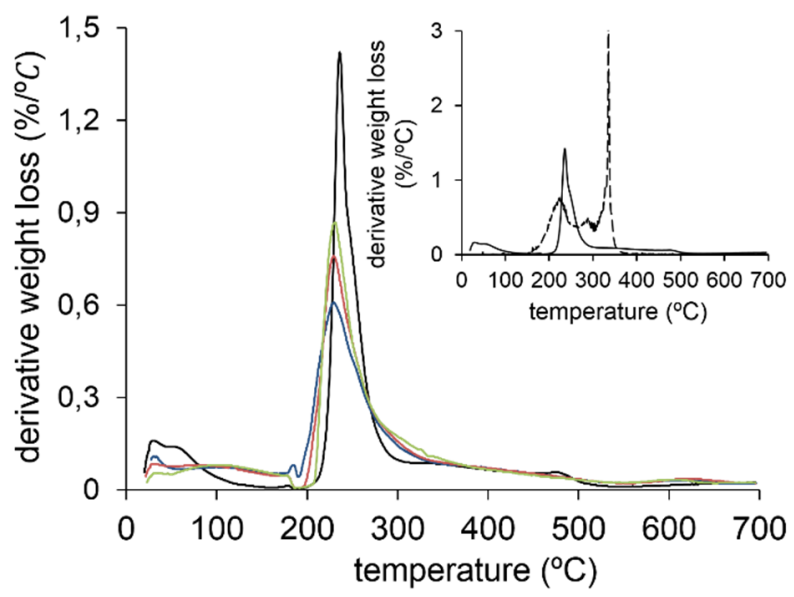

Figure 3. 21. Derivative weight loss thermograms obtained for native HA (black solid line) and the different HA-DVS polymer networks: solv_HA:DVS 1:0.6 (blue line), solv_HA:DVS 1:0.9 (red line) and solv_HA:DVS 1:1.5 (green line). The inset shows a detail of the thermograms of native HA and pDVS (black dotted line).

First of all, it should be mentioned that the two different stages observed in the HA-DVS polymer networks were overlapped at the current rate employed, 10 ${ }^{\circ} \mathrm{C} / \mathrm{min}$, thus the rate was reduced to $1^{\circ} \mathrm{C} / \mathrm{min}$ from room temperature to $180{ }^{\circ} \mathrm{C}$ and the recorded weight was corrected by the data from the calibration at such rate. Figure 3. 21 shows two stages of weight loss in native HA and HA-DVS polymer networks; an initial weight loss stage in the range between room temperature and $180{ }^{\circ} \mathrm{C}$, and a second one from 200 to $700{ }^{\circ} \mathrm{C}$. Focusing the attention in the first weight loss stage (inset in Figure 3.20), it can be observed a similar trend between them but a fast weight loss in native HA compared with HADVS polymer networks. Considering the stabilization of this first stage at $180^{\circ} \mathrm{C}$, these samples show similar values of weight loss, $8.8 \pm 1.9 \stackrel{\circ}{\circ} \mathrm{C}$. The second degradation stage of native HA and HA-DVS polymer networks gave similar trends with a final continuous degradation over the whole temperature range. Therefore the remaining weight at $700^{\circ} \mathrm{C}$ was considered as the residual content, which resulted in invariant values for each sample (30.6 $\pm 2.2 \%)$. On the other hand, the thermograms of derivative weight loss (Figure 3. 21) revealed discrepancies in the maximum rate of weight loss. The maximums observed in the thermograms were correlated with the different degradation stages. Therefore, native HA shows the highest rate of weight loss $\left(1.6 \pm 0.2 \% /{ }^{\circ} \mathrm{C}\right)$ at 237 ${ }^{\circ} \mathrm{C}$ and the HA-DVS polymer networks had lower rates (between the temperature range 205 and $235^{\circ} \mathrm{C}$ ). Furthermore, the maximum rate of weight loss resulted to be higher in the HA-DVS polymer networks containing greater amounts of DVS: $0.8 \pm 0.0 \% / 0 \mathrm{C}$ in solv_HA:DVS $1: 1.5$ samples, $0.8 \pm 0.0 \% / \stackrel{\circ}{ } \mathrm{C}$ in solv_HA:DVS 1:0.9 samples and $0.7 \pm 0.00 \% /{ }^{\circ} \mathrm{C}$ in solv_HA:DVS 1:0.6 samples. 
Thermograms of pDVS (Figure 3. 20 and Figure 3. 21) evidence some differences respect those of the HA-based materials. The thermogram in pDVS shows several overlapped degradations stages between $160 \stackrel{\circ}{\mathrm{C}}$ and $350 \stackrel{\circ}{\circ}$. Focusing the attention in the thermograms of derivative weight loss (Figure 3. 21), three maximums corresponding to different degradation stages were observed: 0.7 $\pm 0.0 \% /{ }^{\circ} \mathrm{C}$ at $226{ }^{\circ} \mathrm{C}, 0.4 \pm 0.0 \% /{ }^{\circ} \mathrm{C}$ at $290{ }^{\circ} \mathrm{C}$ and $3.4 \pm 0.0 \% /{ }^{\circ} \mathrm{C}$ at $335{ }^{\circ} \mathrm{C}$. 


\subsection{Crosslinked hyaluronan scaffolds}

Crosslinked hyaluronan networks and, particularly, HA-DVS polymer networks may constitute a good candidate as implants for tissue engineering purposes. The goals of employing HA materials are the natural origin of HA, biodegradability and stability against aqueous media when crosslinked with DVS. However, the HA-DVS polymer networks lack of internal structure to accomplish an adequate cell colonization, and hence tissue regeneration. In this section, the process to develop HA-DVS scaffolds containing an interconnected porous structure is described, as well as their physicochemical characterization.

\subsubsection{Preparation of scaffolds}

There is a broad range of possibilities to provide scaffolds with an interconnected porous structure. In this case, HA-DVS scaffolds have been developed introducing the crosslinking solution into PEMA templates (see section 2.2.2). In order to obtain an optimal sintering of PEMA beads, the best operating parameters were adjusted and the resulting templates were morphologically analysed by SEM. Moreover, a repetitive process in the development of scaffolds was accomplished subjecting templates to a quality control. This quality control included morphological characterization (Figure 3. 22) and gravimetric determination of the porosity within the template interstices $(24.1 \pm 3.9 \%)$.
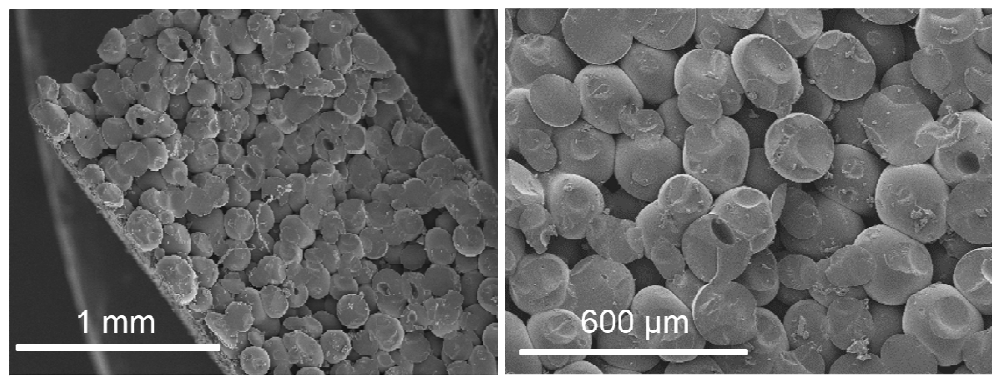

Figure 3. 22. Morphology of the cross-section of PEMA templates obtained by SEM.

The different HA-DVS scaffolds (scaff_HA:DVS 1:0.6, scaff_HA:DVS 1:0.9 and scaff_HA:DVS 1:1.5) were obtained injecting the crosslinking solution, containing the HA and DVS, in the interstices of the PEMA templates. However, in order to reduce the solution viscosity, the concentration of $\mathrm{HA}$ for the crosslinking solution was reduced to $30 \mathrm{mg} / \mathrm{mL}$ (see section 2.2.2). 
With the aim to determine the hydrolysis of $\mathrm{NaOH}$ on $\mathrm{HA}$ at such concentration, the molecular weight distribution of HA under these conditions was compared with the native one using size exclusion chromatography (described in section 2.3).

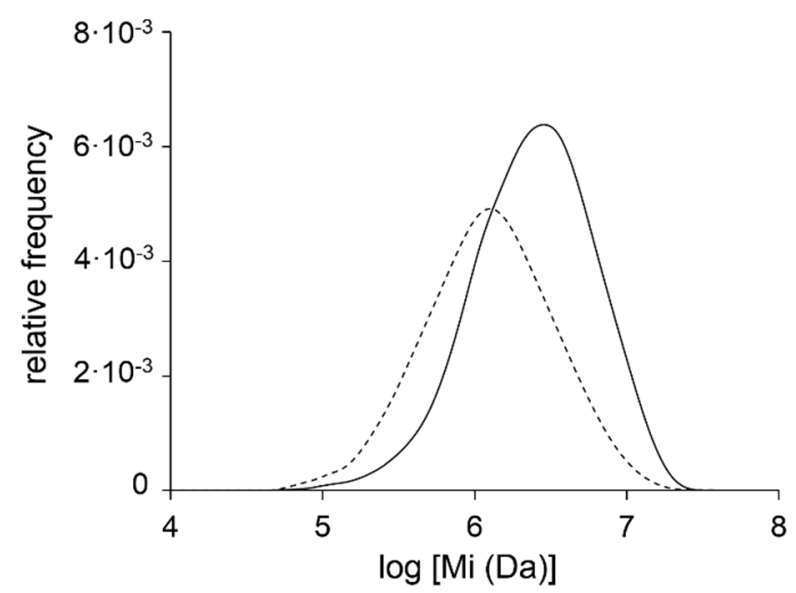

Figure 3. 23. Molecular weight distribution of $\mathrm{HA}$ chains dissolved in $0.15 \mathrm{M} \mathrm{NaCl}$ (solid line) and in a solution of $0.2 \mathrm{M} \mathrm{NaOH}$ for 24 hours (dotted line).

The $\mathrm{HA}$ dissolved in $0.2 \mathrm{M} \mathrm{NaOH}$ at $30 \mathrm{mg} / \mathrm{mL}$ undergoes a considerable reduction of its molecular weight from $1.7 \times 10^{6}$ to $8.0 \times 10^{5} \mathrm{Da}$. However, the PDI value is reduced from 4 to 3.6 and the molecular weight distribution shows a more symmetric trend.

After removing the PEMA beads and salts, and subsequent freeze-drying process, the HA-DVS scaffolds show a fragile structure and a spongy appearance suggesting certain porosity thereof. With the aim to ensure the removal of PEMA beads, scaff_HA:DVS 1:0.9, solv_HA:DVS 1:0.9 samples and PEMA beads were analysed by thermogravimetry. 


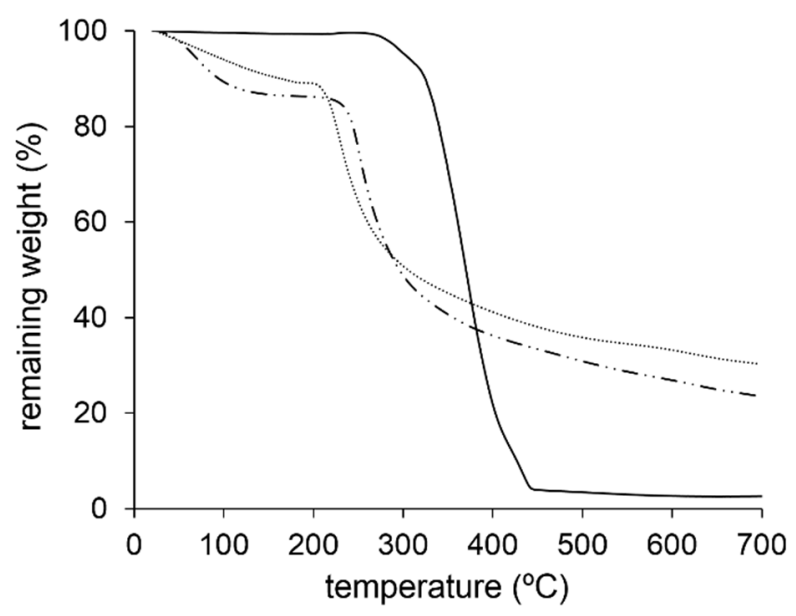

Figure 3. 24. Remaining weight thermograms of PEMA beads (solid line), and solv_HA:DVS 1:0.9 (dotted line) and scaff_HA:DVS 1:0.9 (dashed-dotted line).

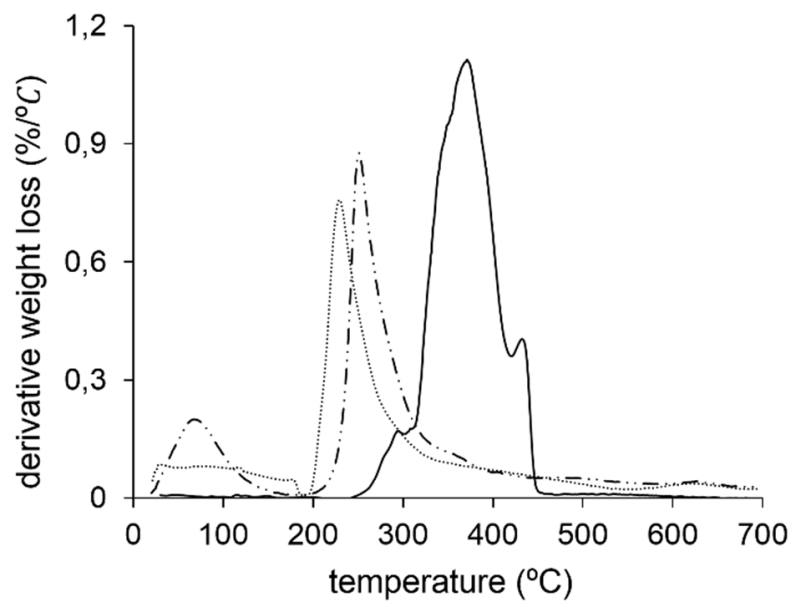

Figure 3. 25. Derivative weight loss thermograms of PEMA beads (solid line), and solv_HA:DVS 1:0.9 (dotted line) and scaff_HA:DVS 1:0.9 samples (dashed-dotted line).

The thermograms of PEMA beads (Figure 3. 24 and Figure 3. 25) resulted in a single and pronounced degradation stage in the temperature range between 300 and $450 \stackrel{\circ}{\circ}$ with the highest rate of weight loss around $370{ }^{\circ} \mathrm{C}$. Moreover, the residual content in PEMA results in values below $5 \%$ of the initial weight. On the contrary, solvent-cast HA-DVS polymer networks and scaffolds show two stages of weight loss: the first one between room temperature and $180{ }^{\circ} \mathrm{C}$ and the second one in the temperature range between 200 and $700{ }^{\circ} \mathrm{C}$. Both HA-DVS based materials show similar trends in the entire range of temperatures. 
However, the first weight loss stage in the scaff_HA:DVS 0.9 samples is faster, while the second one is retarded with respect to the solv_HA:DVS 1:0.9 samples, showing a displacement in the temperature corresponding to the highest degradation rate around $25{ }^{\circ} \mathrm{C}\left(225{ }^{\circ} \mathrm{C}\right.$ and $250{ }^{\circ} \mathrm{C}$ for solv_HA:DVS 1:0.9 and scaff_HA:DVS 1:0.9 samples, respectively).

\subsubsection{Characterization of scaffolds}

\subsubsection{Fourier transformed infrared spectroscopy}

In order to address the crosslinking reaction on the HA-DVS scaffolds, the spectra of pDVS, native HA and the different HA-DVS scaffolds in $\mathrm{KBr}$ were obtained using a smart diffuse reflectance FTIR device (see section 2.3).

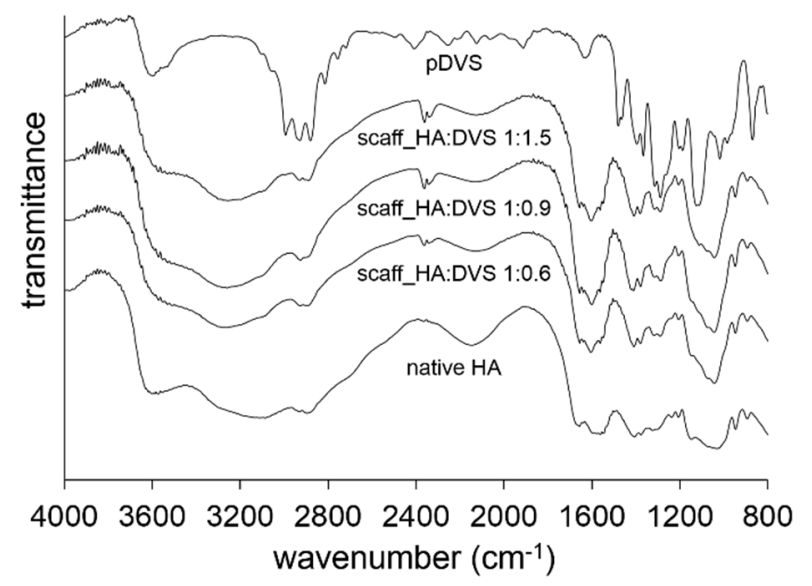

Figure 3. 26. IR spectra of native HA powder, the different HA-DVS scaffolds and pDVS.

Figure 3. 26 shows the different spectra of the mentioned samples. The native HA spectrum shows a broad absorption band between 3800 and $3000 \mathrm{~cm}^{-1}$ attributed to the overlapping of different bond vibrations: the $\mathrm{O}-\mathrm{H}$ stretching of hydroxyl and carboxylic acids and the $\mathrm{N}-\mathrm{H}$ stretching of amides. The multiple peaks in the range between 3000 and $2800 \mathrm{~cm}^{-1}$ are characteristic of the $\mathrm{C}-\mathrm{H}$ stretching of sp3-hybridized carbons. The intense double peak between 1700 and $1500 \mathrm{~cm}^{-1}$ corresponds to the stretching of associated $\mathrm{C}=\mathrm{O}$ groups within carboxylic acids and amide groups (Amide I) and the bending of $\mathrm{N}-\mathrm{H}$ groups (Amide II). The double peak between 1400 and $1380 \mathrm{~cm}^{-1}$ is attributed to the amide group. The intense superposed peaks between $1200-900 \mathrm{~cm}^{-1}$ correspond 
to different bond vibrations ( $\mathrm{C}-\mathrm{O}-\mathrm{C}, \mathrm{C}-\mathrm{O}$ and $\mathrm{C}-\mathrm{O}-\mathrm{H}$ stretching, $\mathrm{N}-\mathrm{H}$ bending, and $\mathrm{O}-\mathrm{H}$ and $\mathrm{C}=\mathrm{O}$ deformation).

The pDVS spectrum is characterized by intense bands between $3000-2800 \mathrm{~cm}^{-1}$ attributed to $\mathrm{C}-\mathrm{H}$ stretching in sp3-hybridized carbons and the two intense bands in $1290 \mathrm{~cm}^{-1}$ and $1110 \mathrm{~cm}^{-1}$ corresponding to the $\mathrm{S}=\mathrm{O}$ asymmetric and symmetric stretching, respectively.

The spectra of the HA-DVS scaffolds show similar characteristic bands than those of native HA, but new bands appeared and some native ones were significantly different. The intense overlapped bands between 1700 and $1500 \mathrm{~cm}^{-}$ ${ }^{1}$ correspond to the $\mathrm{C}=\mathrm{O}$ stretching of carboxylic acids and amides (Amide I), and the $\mathrm{N}-\mathrm{H}$ bending of amide groups (Amide II), all them are characterized by the superposition of three bands showing a symmetric shape. The intense peak of the sulfone symmetric stretching is observed at $1080 \mathrm{~cm}^{-1}$ superposed to characteristic bands of $\mathrm{HA}$, and this band turns more intense with increasing the DVS content. Moreover, the $\mathrm{S}=\mathrm{O}$ asymmetric stretching appears in the spectra of HA-DVS scaffolds as a low-intensity band.

\subsubsection{Sulphur content and degree of crosslinking}

Similarly to the HA-DVS polymer networks, the HA-DVS scaffolds were analysed by elemental analysis with the aim to determine the sulphur content (Table 3. 8), and hence the degree of crosslinking was estimate taking into account the stoichiometry of the crosslinking of HA with DVS (Figure 1. 6).

Table 3. 8. Atomic weight fraction of nitrogen, carbon, hydrogen and sulphur in the HA-DVS scaffolds and native HA.

\begin{tabular}{ccccc}
\hline \multirow{2}{*}{ Sample } & \multicolumn{4}{c}{ Atomic weight fraction (in percentage) } \\
\cline { 2 - 5 } & Nitrogen & Carbon & Hydrogen & sulphur \\
\hline native HA & 3.2 & 32.7 & 9.1 & 0.0 \\
scaff_HA:DVS 1:0.6 & 2.9 & 32.4 & 8.0 & 0.6 \\
scaff_HA:DVS 1:0.9 & 2.9 & 32.1 & 8.4 & 0.9 \\
scaff_HA:DVS 1:1.5 & 2.7 & 32.4 & 8.5 & 1.7 \\
\hline
\end{tabular}


The sulphur content resulted in higher values in the HA-DVS scaffolds with higher amounts of DVS. Therefore, the sulphur content was compared with the carbon and nitrogen content and, taking into account the stoichiometry of the crosslinking of HA with DVS, the different parameters of the crosslinking (degree of crosslinking, $\bar{M}_{c}$ and $\bar{d}$ ) were determined (Table 3. 9).

Table 3. 9. Parameters of the HA crosslinked scaffolds with DVS determined by elemental analysis.

\begin{tabular}{cccc}
\hline Composition & $\begin{array}{c}\text { Scaff_HA:DVS } \\
\mathbf{1 : 0 . 6}\end{array}$ & $\begin{array}{c}\text { scaff_HA:DVS } \\
\mathbf{1 : 0 . 9}\end{array}$ & $\begin{array}{c}\text { scaff_HA:DVS } \\
\mathbf{1 : 1 . 5}\end{array}$ \\
\hline degree of crosslinking (\%) & 18.4 & 20.9 & 42.6 \\
$\overline{\mathrm{M}}_{\mathrm{c}}(\mathrm{kDa})$ & 2.2 & 1.9 & 0.9 \\
$\overline{\mathrm{d}}$ & 5.4 & 4.8 & 2.3 \\
\hline
\end{tabular}

The parameters collected in Table 3. 9 show that the higher contents of DVS increase the degree of crosslinking in the HA-DVS scaffolds, and hence the HA molecules contain a lower molecular weight and disaccharide units between crosslinks.

\subsubsection{Morphological characterization}

The morphology of the different HA-DVS scaffolds was evaluated by SEM with the aim to assess their adequate interconnected porous structure. At the beginning, the HA-DVS scaffolds were swollen in deionized water until the equilibrium swelling ( 3 days). Then, the different scaffolds were subjected to a freeze-drying process and a posterior cryofracture obtaining their vertical crosssection. 

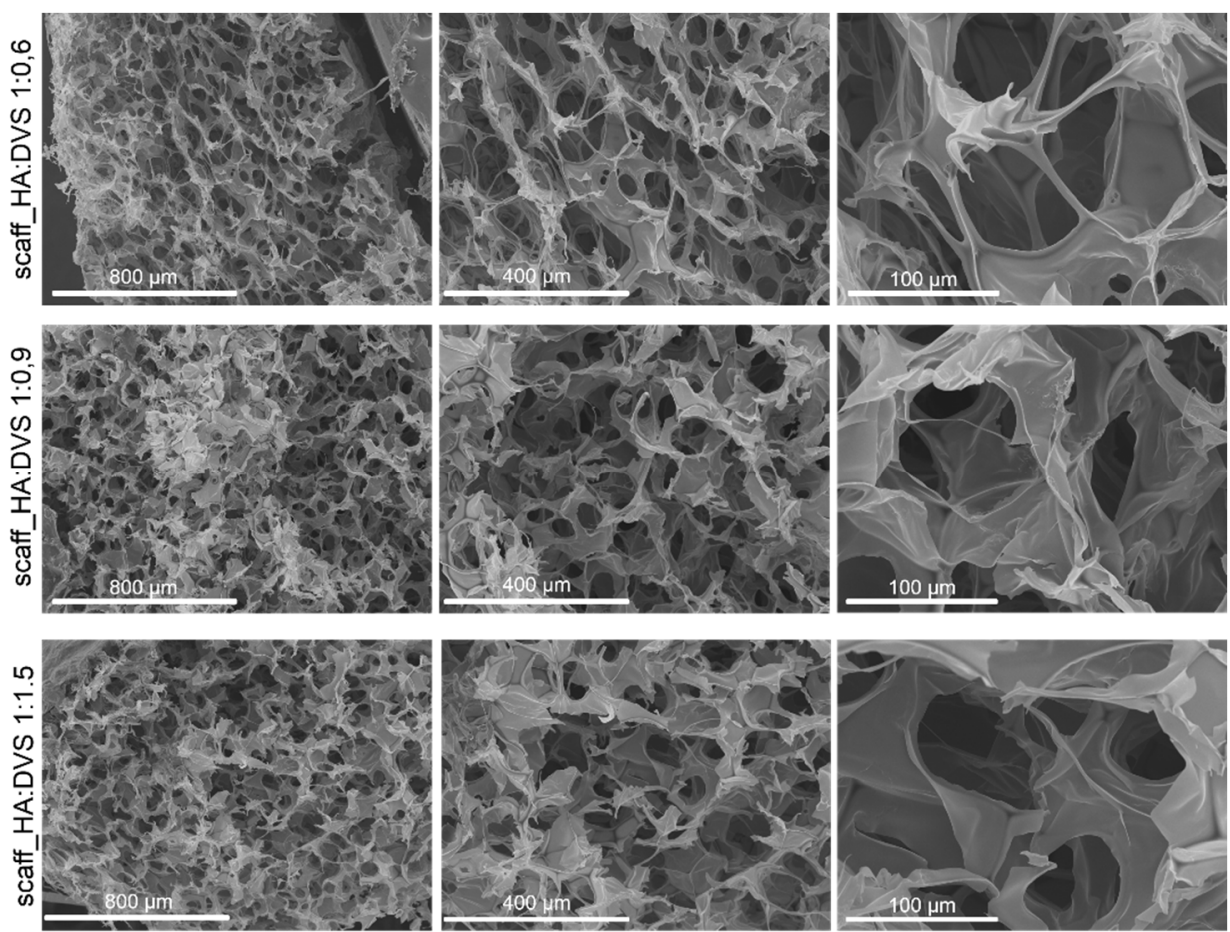

Figure 3. 27. Images of the inner structure in the different HA-DVS scaffolds obtained by SEM.

The internal structure (Figure 3. 27) and surface (data not shown) between the different scaffolds showed no significant differences: in all the cases HA-DVS scaffolds showed a highly interconnected and porous structure along their vertical cross-section. The porosity due to the PEMA beads was eclipsed by the sheetlike structure of the bulk material. The thickness of these sheets was measured by image software resulting in sizes below $5 \mu \mathrm{m}$. Moreover, the inner walls of the scaffolds resulted to be uniform without displaying microporosity. On the other hand, the interconnection between pores resulted to be spherical and oval with variable sizes showing no significant differences between compositions: 76.7 $\pm 20.9 \mu \mathrm{m}$ in scaff_HA:DVS 1:0.6, 76.0 $\pm 22.1 \mu \mathrm{m}$ in scaff_HA:DVS 1:0.9 and 82.8 $\pm 25.7 \mu \mathrm{m}$ in scaff_HA:DVS 1:1.5. 


\subsubsection{Porosity}

In order to obtain the macroporosity (large pores) due to the PEMA template and the microporosity (small pores) in the bulk material due to the freeze-drying process, the porosity of the different HA-DVS scaffolds was determined by different methods (see section 2.3).

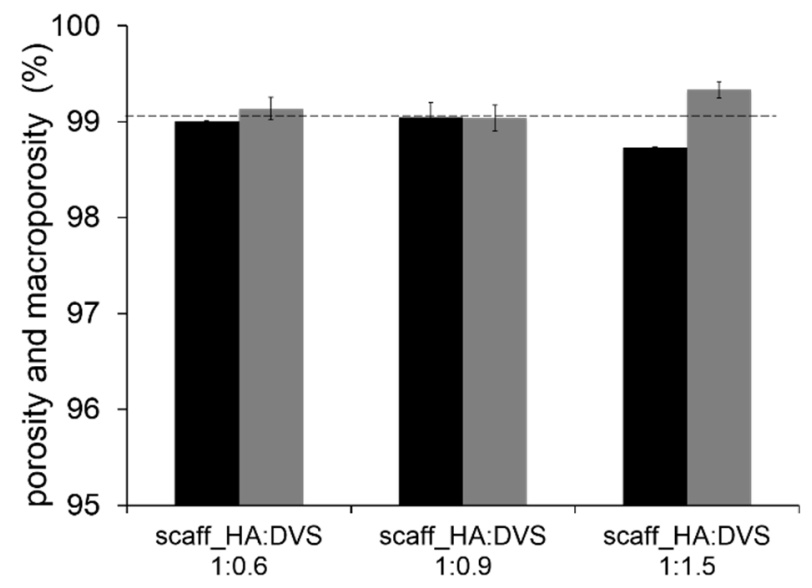

Figure 3. 28. Porosity due to large pores (black bars) and including large and small pores (grey bars) in the different HA-DVS scaffolds. The horizontal dashed line shows the averaged porosity between samples and method.

Figure 3.28 shows that the HA-DVS scaffolds contain a very high porosity with no significant differences between compositions. Moreover, the porosity due to large and small pores resulted in similar values for each composition, except for the scaff_HA:DVS 1:1.5 sample. Despite the discrepancies found in the scaff_HA:DVS 1:1.5 composition, the porosity value $(99.0 \pm 0.1 \%)$ was considered similar for all the compositions and the effect of large and small pores in such value was neglected. 


\subsubsection{Swelling experiments}

In order to compare the swelling on scaffolds and polymer networks, WI_HA-DVS samples were subjected to similar washing and drying processes (see section 2.2.2). The $E W C$ values of the different materials immersed in $0.15 \mathrm{M} \mathrm{PBS}$ and deionized water for 3 days were determined (see section 2.3).

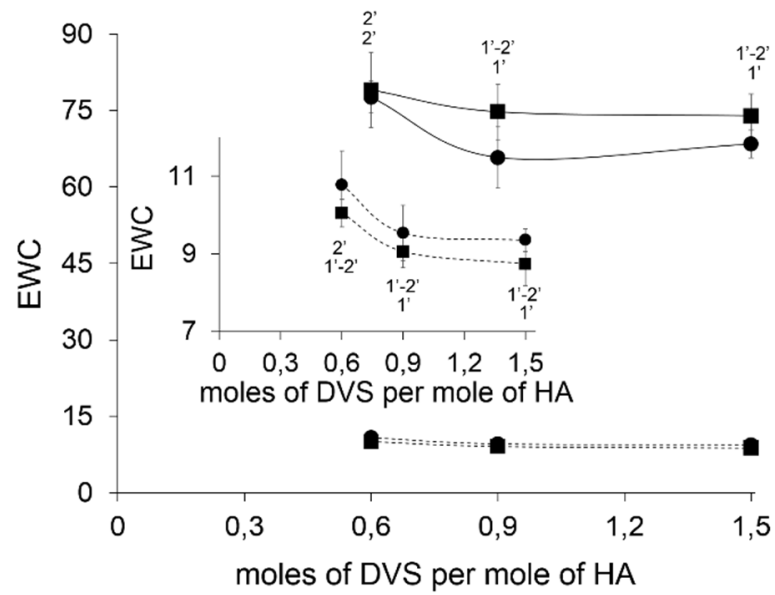

Figure 3. 29. The EWC value of HA-DVS scaffolds (solid lines) and WI_HA-DVS samples (dashed lines) in immersion of $0.15 \mathrm{M}$ PBS (squares) and de-ionized water (circles). Inset shows a zoom of the EWC value in WI_HA-DVS samples. Vertical bars represent the standard deviation of each experience.

As can be observed in Figure 3. 29, the swelling degree in each media resulted much higher on HA-DVS scaffolds (EWC values ranging from 65 to 80 ) than in WI_HA-DVS samples (EWC values ranging from 5 to 11). In general, the EWC of the HA-DVS scaffolds resulted in higher values when immersed in $0.15 \mathrm{M}$ PBS than those obtained for deionized water. Moreover, the compositions with lower amounts of DVS displayed higher EWC values; however, these discrepancies showed no significant differences. On the contrary, the EWC in WI_HA-DVS samples showed higher values when immersed in deionized water (values ranging from 9 to 11) than those determined for $0.15 \mathrm{M}$ PBS (values around 5). In the case of WI_HA-DVS samples, differences in swelling depending on the immersed medium showed significant differences. However, as the case of HADVS scaffolds, the EWC values between the different WI_HA-DVS samples resulted to be similar in both media. 


\subsubsection{Compression tests}

Compression tests of HA-DVS scaffolds and WI_HA-DVS samples at equilibrium swelling of $0.15 \mathrm{M}$ PBS were conducted with the aim to compare the mechanical properties of the HA-DVS bulk material and scaffolds (see section 2.3). In order to determine the compatibility of the materials for future brain implants from the point of view of their mechanical properties, compression tests of brain tissue of postnatal rats were conducted in a similar way.

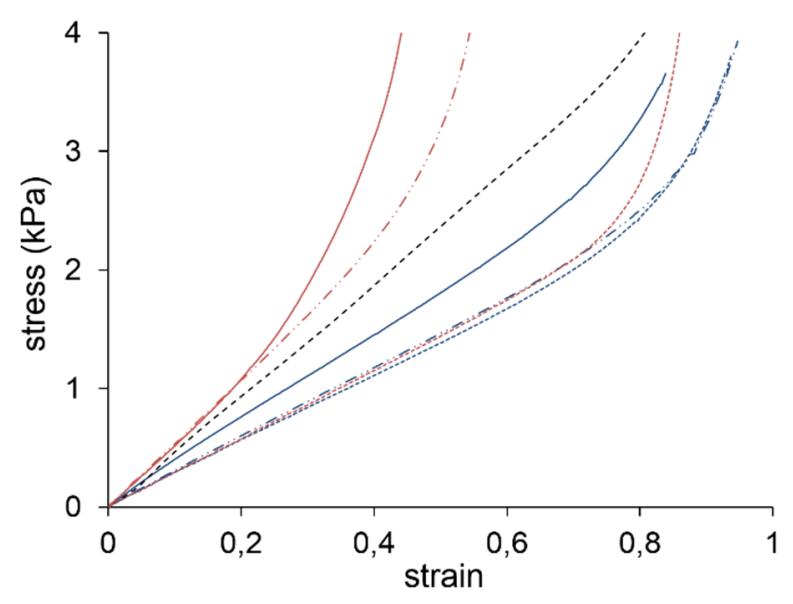

Figure 3. 30. Stress-strain curves of the different WI_HA:DVS compositions (red lines) (WI_HA:DVS 1:0.6 (dotted line), WI_HA:DVS 1:0.9 (dashed line) and WI_HA:DVS 1:1.5 (solid line)), brain tissue (dashed black line) and HA-DVS scaffolds (blue lines) (scaff_HA:DVS 1:0.6 (dotted line), scaff_HA:DVS 1:0.9 (dashed line) and scaff_HA:DVS 1:1.5 (solid line)). Samples were measured at equilibrium swelling in $0.15 \mathrm{M}$ PBS.

Figure 3. 30 shows similar trends for the stress-strain curves of the different materials; an initial and relative long elastic zone and after a densification region a second elastic region, in which the stress increases faster without noticeable increasing of strain. In the case of the brain tissue, the curve does not show a clearly identifiable densification region. The stress-strain curves of HA-DVS scaffolds evidence a very high first elastic region, reaching great strains (up to 0.8 ) in the stress range studied. In the Figure 3.30 can be observed that scaff_HA:DVS 1:0.6 and scaff_HA:DVS 1:0.9 samples show similar curves reaching strains near 1 , while the scaff_HA:DVS 1:1.5 sample shows a relative less soft behaviour reaching a strain near 0.8 . Moreover, the brain tissue shows a less soft behaviour than all the scaffolds compositions, showing a more similar behaviour with respect to the scaff_HA:DVS 1:1.5 sample. On the other hand, the stress-strain curves of WI_HA-DVS samples show a shorter first elastic region 
and the stress in the second elastic region increases faster compared with that observed in the HA-DVS scaffolds. Therefore, the WI_HA-DVS samples under compression reached lower strains in the range studied compared with scaffolds: values around 0.4 for WI_HA:DVS 1:1.5, 0.5 for WI_HA:DVS 1:0.9 and 0.8 for WI_HA:DVS 1:0.6. The WI_HA:DVS 1:0.6 sample shows a similar initial slope to those of scaff_HA:DVS 1:0.6 and scaff_HA:DVS 1:0.9 samples, but it resulted in a faster increasing of stress along the second elastic region.

In order to compare the mechanical properties of the different materials and brain tissue, different parameters were calculated from the stress strain curves: the initial modulus $\left(E_{0}\right)$, final modulus $\left(E_{f}\right)$ and final strain $\left(\varepsilon_{f}\right)$. The $E_{0}$ value was determined from the initial slope from strains between 0 and 0.1 . The $E_{f}$ value was determined from the slope in the region compressing stresses between 3 and $3.5 \mathrm{kPa}$ and from the intersection of this slope with the strain axis was defined the $\varepsilon_{f}$ value.
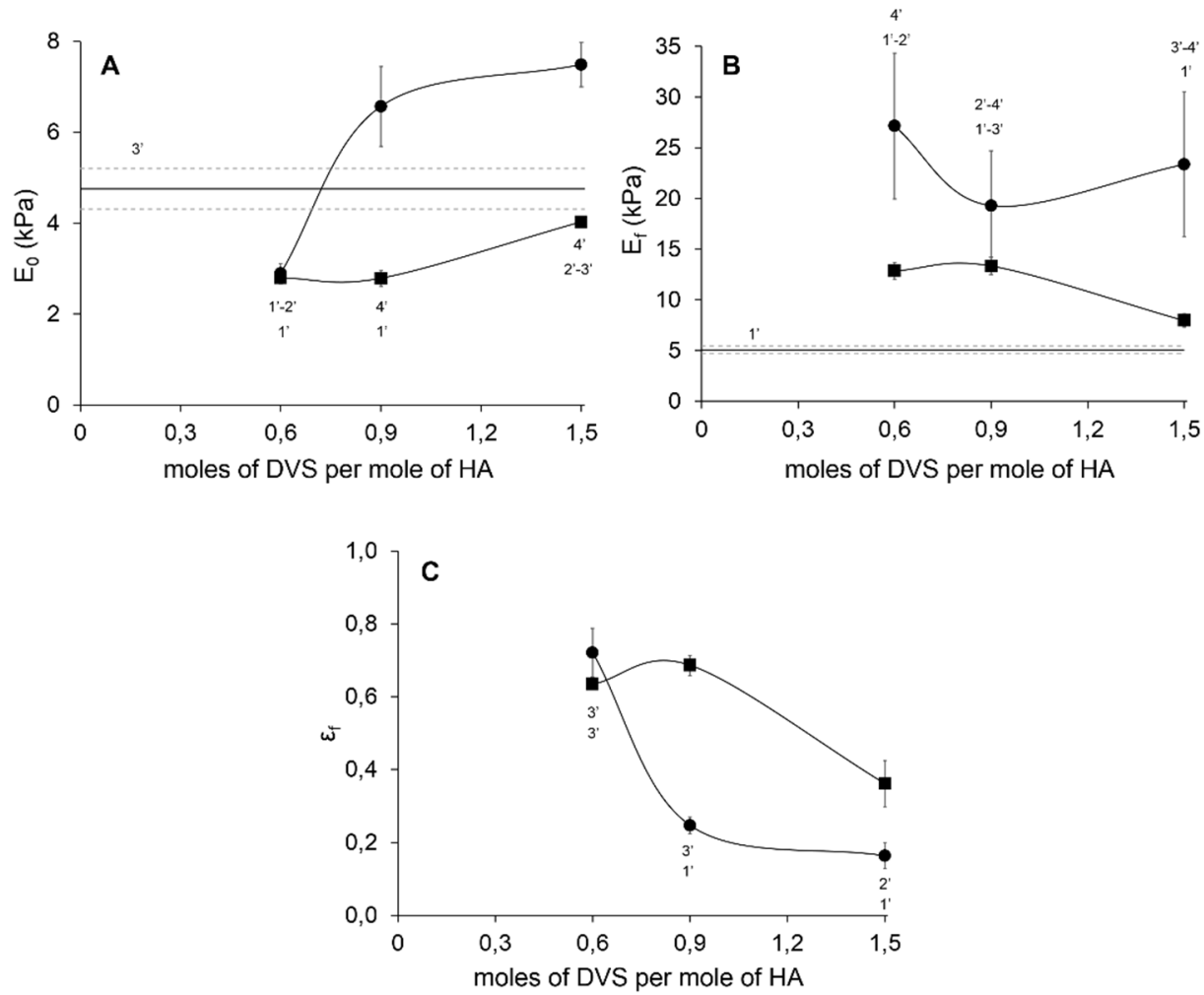

Figure 3. 31. Parameters of WI_HA-DVS samples (circles), HA-DVS scaffolds (squares) and rat brain (horizontal solid line) obtained from the compression tests. A) Initial modulus, B) final modulus and C) final strain. 
With respect to the initial modulus (Figure 3. 31-A), the scaffolds show values in the range between 2 and $4 \mathrm{kPa}$, while this modulus is slightly higher for WI_HADVS samples showing values from 2 to $8 \mathrm{kPa}$. These differences between samples become significant with increasing the DVS content. However, in both materials, the value of $E_{0}$ slightly increases in samples with higher amounts of DVS. Moreover, the different HA-DVS scaffolds show smaller initial modulus values than brain tissue $(4.8 \pm 0.4 \mathrm{kPa})$ arising more similar values with scaff_HA:DVS 1:1.5 samples $(4.0 \pm 0.0 \mathrm{kPa})$.

The Figure 3. 31-B shows that the $E_{f}$ values of HA-DVS scaffolds (from 14 to 35 $\mathrm{kPa}$ ) resulted lower than that of WI_HA-DVS samples (from 7 to $14 \mathrm{kPa}$ ). The $E_{f}$ values resulted in a different trend compared with that of the initial modulus: the higher the molar ratio of DVS to HA in the samples, the lower the $E_{f}$ values. However, differences in $E_{f}$ in the scaffolds resulted to not be significant between compositions, while WI_HA-DVS samples showed different $E_{f}$ values only for WI_HA:DVS 1:1.5 samples. In this case, brain tissue showed lower values of $E_{f}$ $(5.1 \pm 0.4 \mathrm{kPa})$ than the different materials, and differences between the values of initial modulus and $E_{f}$ resulted to be minimal. On the other hand, the $\varepsilon_{f}$ revealed in general higher values in scaffolds ranging from 0.6 to 0.7 in scaff_HA:DVS 1:0.6 and scaff_HA:DVS 1:0.9 samples and values from 0.3 to 0.5 in scaff_HA:DVS 1:1.5 samples. In the case of WI_HA-DVS samples the values of $\varepsilon_{f}$ resulted to have a maximum in WI_HA:DVS 1:0.6 samples (0.7) with a significant decreasing in WI_HA:DVS 1:0.9 and WI_HA:DVS 1:1.5 samples $(0.25$ and 0.16 , respectively).

\subsubsection{Thermogravimetric analysis}

In order to assess the thermal degradation stages, the different HA-DVS scaffolds were subjected to a thermogravimetric analysis from room temperature to $700{ }^{\circ} \mathrm{C}$ (see section 2.3). Additionally, the native HA was conducted in similar conditions with the aim to compare its thermal stability with that of the scaffolds. Thermogravimetric analyses allow obtaining two different thermograms showing the remaining weight and the derivative weight loss as functions of the temperature. 


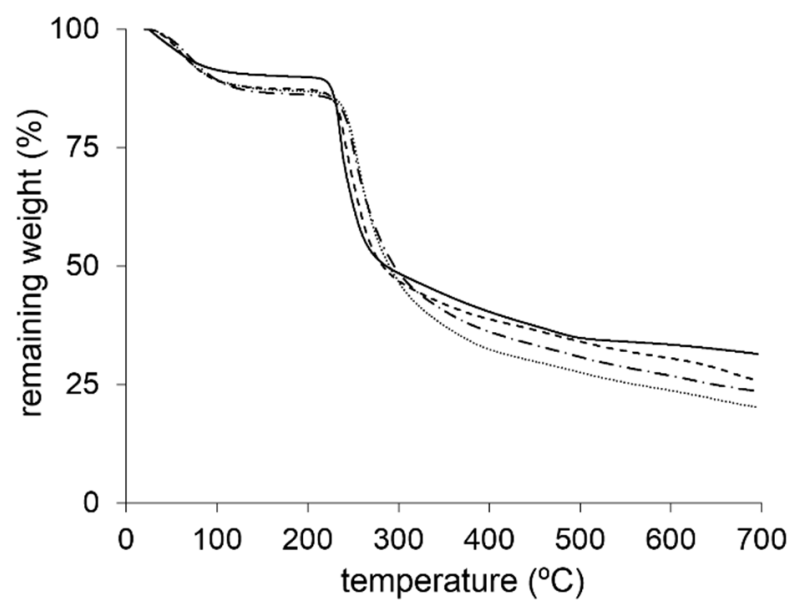

Figure 3. 32. Remaining weight thermograms of the native HA (solid line) and the different HA-DVS scaffolds: scaff_HA:DVS 1:0.6 (dashed line), scaff_HA:DVS 1:0.9 (dashed-dotted line) and scaff_HA:DVS 1:1.5 (dotted line).

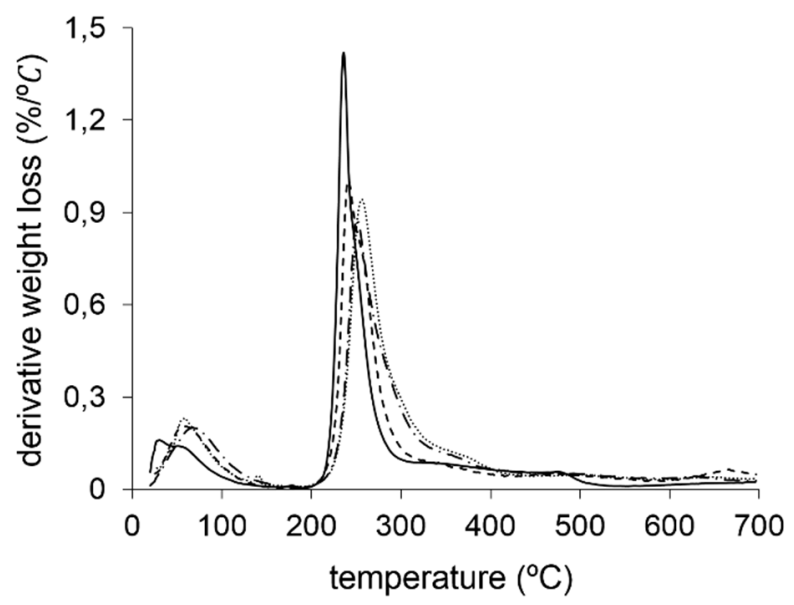

Figure 3. 33. Derivative weight loss thermograms of the native HA (solid line) and the different HADVS scaffolds: scaff_HA:DVS 1:0.6 (dashed line), scaff_HA:DVS 1:0.9 (dashed-dotted line) and scaff_HA:DVS 1:1.5 (dotted line).

Figure 3. 32 and Figure 3. 33 show that the different materials and native HA yield two differentiated stages of weight loss; the first one occurs in the temperature range between room temperature and $180^{\circ} \mathrm{C}$, and the second one from $225^{\circ} \mathrm{C}$ to the final temperature recorded $\left(700^{\circ} \mathrm{C}\right)$. As can be observed in the thermograms, some differences were evidenced between native HA and HA-DVS scaffolds; however, differences between HA-DVS scaffolds compositions resulted to be non-significant different and could be neglected. Therefore, the 
thermal parameters of the HA-DVS scaffolds were averaged between the different compositions. In the first weight loss stage, the different HA-DVS scaffolds and native HA show similar trends, however, at the end of this first stage, native HA shows lower weight loss $(8.1 \pm 0.6 \%)$ than the different HA-DVS scaffolds $(13.0 \pm 1.7 \%)$.

In the second degradation stage, the thermograms of native HA and HA-DVS scaffolds give similar trends showing continuity in the loss of weight at $700 \stackrel{\circ}{\circ}$. The percentage of residual species, determined from the remaining weight at 700 ${ }^{\circ} \mathrm{C}$, gave similar values in the different HA-DVS scaffolds (22.9 $\left.\pm 2.4 \%\right)$, while this value resulted in higher values in native $\mathrm{HA}(31.7 \pm 4.0 \%)$.

On the other hand, the thermograms of derivative weight loss (Figure 3. 33) arise discrepancies in the rate of weight loss between the HA-DVS scaffolds and native $\mathrm{HA}$. The maximum degradation rate corresponding to the first stage of degradation shows no significant differences between the HA-DVS scaffolds and native $\mathrm{HA}$, showing an averaged value of $0.2 \pm 0.0 \% /{ }^{\circ} \mathrm{C}$. However, in the second degradation stage, a noticeable difference between the maximum degradation rate of native HA $\left(1.6 \pm 0.2 \% /{ }^{\circ} \mathrm{C}\right)$ and HA-DVS scaffolds $\left(1.0 \pm 0.2 \% /{ }^{\circ} \mathrm{C}\right)$ can be observed. 


\subsection{Poly(methacrylated-co-ethyl acrylate) copolymer networks}

The methacrylated HA macromer, MeHA, was synthesized by the esterification reaction between native $\mathrm{HA}$ and MA. In order to tailor the swelling degree, mechanical properties and degradability, the MeHA macromer was copolymerized with EA obtaining semi-degradable copolymers. The EA was chosen as monomer because of the biostable nature of PEA polymer networks possess relative low mechanical properties and low swelling ratios compared with other similar polymers, which make it attractive on obtaining materials with controllable properties for soft tissue engineering, and particularly for neural regeneration purposes.

\subsubsection{Methacrylated hyaluronan macromer}

\subsubsection{Synthesis of methacrylated hyaluronan macromer}

In order to obtain poly(MeHA-co-EA) copolymer networks, HA was modified leading to chains with vinyl groups (described in section 2.2.3). Therefore, methacrylated hyaluronan (MeHA) was synthesized by adding 20 -fold excess of methacrylic anhydride (MA) to a solution of $\mathrm{HA}$ in $0.2 \mathrm{M} \mathrm{NaOH}$. The excess of $M A$ is required due to the low efficiency of this reaction in aqueous solutions. Moreover, the basic media prevents the formation of excessive methacrylic acid along the reaction. The MA was added dropwise in the HA solution under vigorous stirring due to the low solubility of this reagent in aqueous media and avoiding phase separation as possible.

After the reaction, the impure product resulted in a white, viscous and homogenous solution. During the purification process, and particularly in the precipitation with acetone, the product became a solid and white paste hindering its posterior solubility in water. However, the subsequent purification steps removed the insoluble components of the product. After the freeze-drying process, the product showed a white powdery appearance similar to the native HA. The reaction yield, amount of product obtained with respect to the initial HA employed, was calculated from three different batches resulting in an averaged value of $56.6 \pm 6.9 \%$.

In order to determine changes in the molecular weight, the MeHA product and native $\mathrm{HA}$ were dissolved in $0.15 \mathrm{M} \mathrm{NaCl}$ at a concentration of $1 \mathrm{mg} / \mathrm{mL}$, and these solutions were passed through gel permeation columns (see section 2.3). The different parameters ( $\mathrm{Mw}, \mathrm{Mn}$ and $\mathrm{PDI}$ ) and the molecular weight distribution of native and modified HA were determined similarly to those presented in sections 3.1.1 and 3.2.1. 


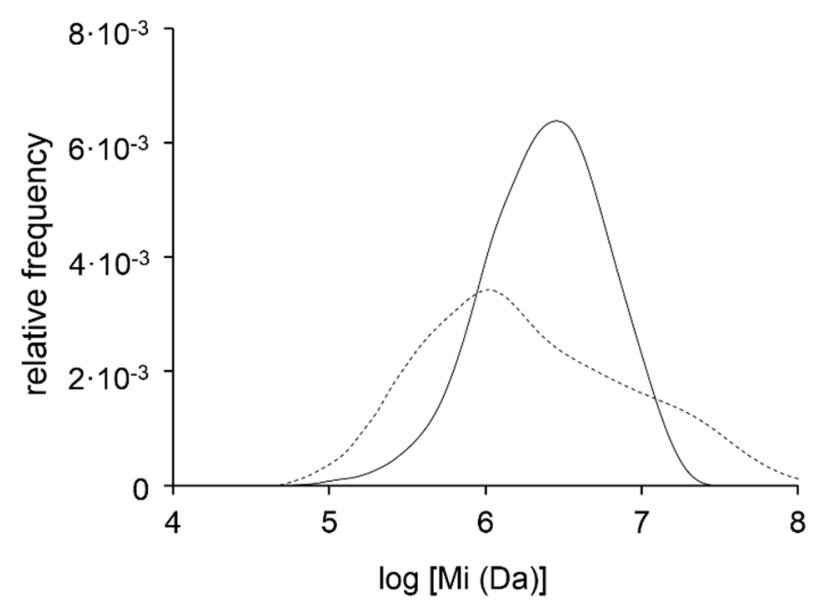

Figure 3. 34. Molecular weight distribution of HA (solid line) and MeHA (dashed line).

The Figure 3. 34 shows the differences of the molecular weight distribution between native HA and MeHA. The native HA resulted in a $M w$ value of $1.74 \mathrm{MDa}$ with a PDI value of 4 . However, the MeHA product resulted in higher Mw (2.48 $\mathrm{MDa}$ ) and $\mathrm{PDI}(13.3)$ values.

\subsubsection{Fourier transformed infrared spectroscopy}

In order to assess the methacrylation of $\mathrm{HA}$, the spectra of native $\mathrm{HA}$ and MeHA powders were obtained by FTIR spectroscopy (see section 2.3). The MeHA spectrum was compared with that of native HA. 


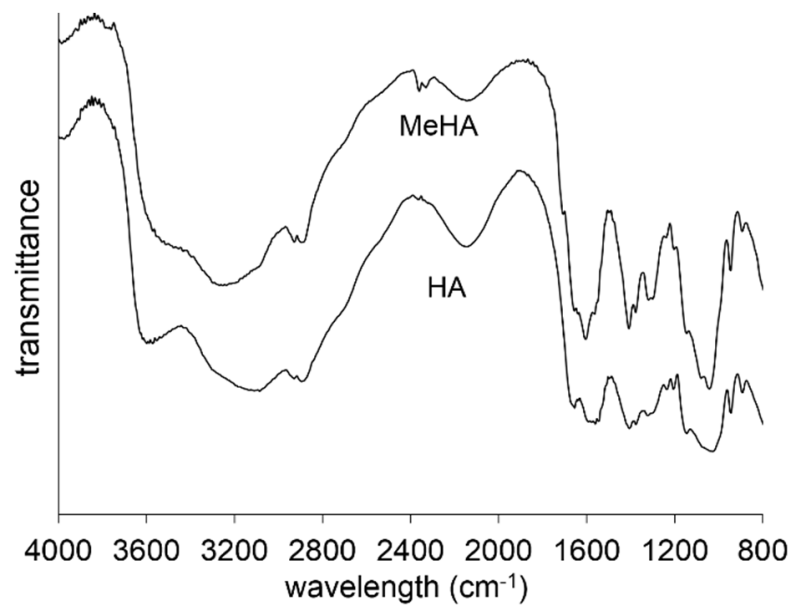

Figure 3. 35. IR spectra of native HA powder and MeHA.

The characteristic IR absorption bands of native HA were described in section 3.2.2.1. The broad band between $3600-3000 \mathrm{~cm}^{-1}$ is attributed to the $\mathrm{O}-\mathrm{H}$ and $\mathrm{N}$ $\mathrm{H}$ stretching of associated hydroxyls in acids and amides, respectively. The multiple peaks in the range $3000-2800 \mathrm{~cm}^{-1}$ are characteristic of the $\mathrm{C}-\mathrm{H}$ stretching. The overlapped bands between 1800 and $1600 \mathrm{~cm}^{-1}$ correspond to $\mathrm{C}=\mathrm{O}$ stretching of carboxylic acids and amides (Amide I) and $\mathrm{N}-\mathrm{H}$ bending of amide groups (Amide II). The peak at $1400 \mathrm{~cm}^{-1}$ is attributed to the $\mathrm{C}-\mathrm{N}$ stretching. The intense overlapped peaks between $1200-900 \mathrm{~cm}^{-1}$ correspond to other bond vibrations (C-O-C, C-O and $\mathrm{C}-\mathrm{O}-\mathrm{H}$ stretching, $\mathrm{N}-\mathrm{H}$ bending, and $\mathrm{O}-\mathrm{H}$ and $\mathrm{C}=\mathrm{O}$ deformation).

On the other hand, the spectrum of MeHA powder (Figure 3. 35) shows similarities with that of the native HA. However, with a detailed study of both spectra small differences may be appreciated. The broad band between 3600 and $2400 \mathrm{~cm}^{-1}$ revealed maximums at different frequencies in native HA (3100 $\left.\mathrm{cm}^{-1}\right)$ and MeHA $\left(3300 \mathrm{~cm}^{-1}\right)$, and the band of $3600 \mathrm{~cm}^{-1}$ corresponding to free O$\mathrm{H}$ stretching becomes less intense. The overlapped bands between 1800 and $1600 \mathrm{~cm}^{-1}$ present different shapes; in the case of native HA the bands corresponding to the $\mathrm{C}=\mathrm{O}$ stretching of carboxylic acids and amides shows an asymmetric distribution, while these bands in the MeHA resulted in a higher symmetry showing an intense peak centred at $1600 \mathrm{~cm}^{-1}$. The intense overlapped peaks between $1200-900 \mathrm{~cm}^{-1}$ show an increase of the intensity at $1000 \mathrm{~cm}^{-1}$, which is attributed with the $\mathrm{C}=\mathrm{C}-\mathrm{H}$ bending. 


\subsubsection{Determination of the degree of methacrylation}

In order to assess the efficiency of methacrylation and quantify the degree of methacrylation of the MeHA macromer, the ${ }^{1} \mathrm{H}-\mathrm{NMR}$ spectra of native HA and MeHA were compared. The ${ }^{1} \mathrm{H}-\mathrm{NMR}$ spectrum of MeHA was obtained from a homogeneous mixture of powder from three different batches.

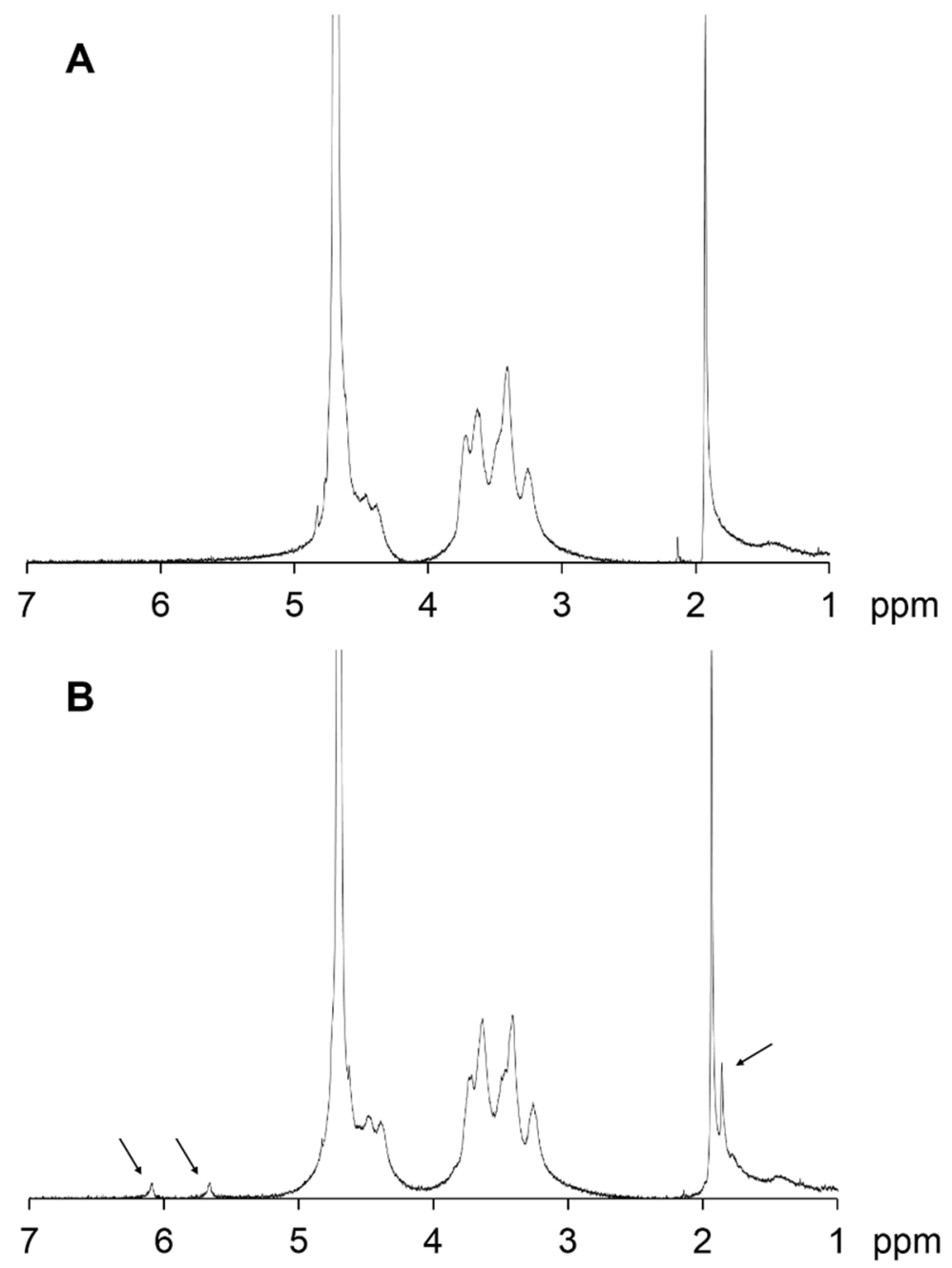

Figure 3. 36. ${ }^{1} \mathrm{H}-\mathrm{NMR}$ spectrum of $\mathrm{HA}(\mathrm{A})$ and $\mathrm{MeHA}(\mathrm{B})$. The arrows show the characteristic peaks of methacrylate groups. 
The ${ }^{1} \mathrm{H}-\mathrm{NMR}$ spectrum (Figure 3.36 ) of native HA revealed signals at three different regions. The first one between 1 and 2 ppm shows an intense singlet associated with the three protons of the methyl group in acetamide. The second region lies between 3 and $4 \mathrm{ppm}$ and contains a complex pattern that is associated with several protons of the HA backbone. Finally, the third region comprised between 4 and $4.5 \mathrm{ppm}$ shows two peaks overlapped with the intense $\mathrm{D}_{2} \mathrm{O}$ signal, and these peaks are attributed to hydrogens in the $\mathrm{HA}$ backbone that are close to electronegative groups (ether linkages). On the other hand, the MeHA spectrum revealed three new bands attributed to the methacrylate group: at 6.10 and $5.60 \mathrm{ppm}$ associated with the protons within the vinyl group and at $1.85 \mathrm{ppm}$ attributed to the three protons in the allyl group.

The degree of methacrylation (methacrylate groups per HA disaccharide unit) was determined comparing the relative integrated intensities of the five methacrylate protons in MeHA (peaks at 6.10, 5.60 and $1.85 \mathrm{ppm}$ ) and the three methyl protons within the $\mathrm{HA}$ acetamide (peak at $1.93 \mathrm{ppm}$ ), resulting in a value of $12.5 \%$.

\subsubsection{Synthesis of poly(methacrylated-co-ethyl acrylate) copolymer networks}

After addressing the methacrylation of $\mathrm{HA}$, the macromer was employed in the copolymerization reaction of MeHA and EA with the aim to obtain poly(MeHA-coEA) copolymer networks. In order to evaluate differences in the mechanical properties and the stability of the poly(MeHA-co-EA) copolymer networks, copolymerization was carried out using different weight ratios of MeHA to EA, including the homopolymer networks (see Table 2. 3). Due to the different polarity of both components, monomers were dissolved in a mixture of solvents (DMF/0.15 M PBS, 1/1 (v/v)). Therefore, salts in the polymer solutions were added with the aim of decreasing the viscosity of MeHA. In addition, the low solubility of both monomers in the solution limited the monomeric concentration, and the copolymerization reactions were carried out as very dilute ones. In order to assess the reaction of the macromer and monomer chains, MBAA ( $5 \%$ with respect to the amount of MeHA and/or EA in the copolymerization reaction) was added as crosslinking agent and the reaction was performed with a considerable amount of thermal initiator ( $1 \%$ with respect to the amount of MeHA and/or EA in the copolymerization reaction).

After the thermal copolymerization, the poly(MeHA-co-EA) copolymer networks showed a homogeneous, whitish and soft appearance. During the washing steps the poly(MeHA-co-EA) compositions with larger amounts of MeHA lead to a transparent hydrogel, while the ones with larger amounts of EA resulted to give a whitish colour. After the purification and freeze-drying process, the xerogels 
where characterized by different techniques with the aim to determine the composition and phase distribution of both components.

\subsubsection{Fourier transformed infrared spectroscopy}

In order to assess the incorporation of polymerized MeHA and PEA in the poly(MeHA-co-EA) copolymer networks, each composition was chemically studied by Fourier transformed infrared spectroscopy (FTIR) (see section 2.3). In order to determine variations in the infrared bands due to the different chemical groups of each component, spectra of different copolymer networks and those of the homopolymer networks were recorded.

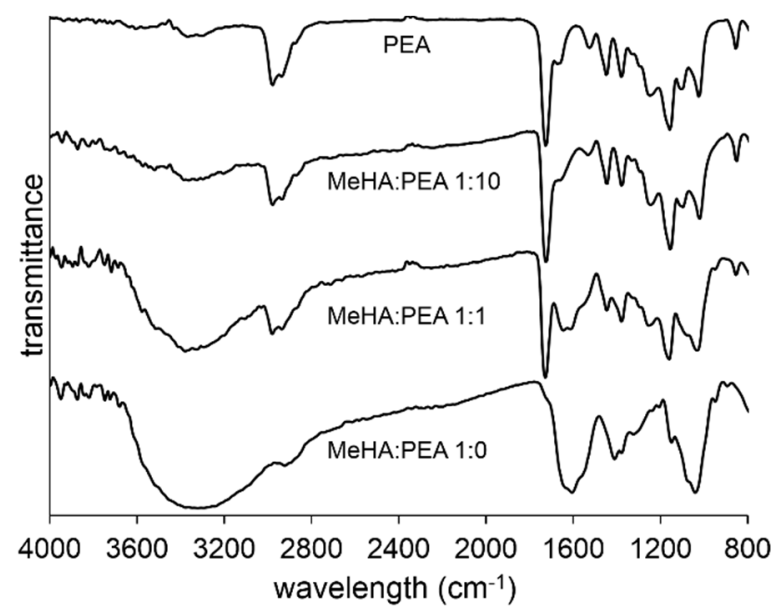

Figure 3. 37. IR spectra of the different poly(MeHA-co-EA) copolymer and homopolymer networks.

Figure 3. 37 shows that the spectrum of MeHA:PEA 1:0 samples contains a broad band between $3800-2800 \mathrm{~cm}^{-1}$ attributed to different bond vibrations: associated $\mathrm{O}-\mathrm{H}$ stretching of hydroxyl groups and associated $\mathrm{N}-\mathrm{H}$ stretching of amide groups. The bands in the range between 3000 and $2800 \mathrm{~cm}^{-1}$ are characteristic of $\mathrm{C}-\mathrm{H}$ stretching in sp3-hybridized carbons. The broad band between 1700 and $1500 \mathrm{~cm}^{-1}$ corresponds to the overlapping of the $\mathrm{C}=\mathrm{O}$ stretching within the carboxylate and amide (Amide I), and the $\mathrm{N}-\mathrm{H}$ bending of amide groups (Amide II). The peak at $1400 \mathrm{~cm}^{-1}$ is characteristic of the $\mathrm{C}-\mathrm{N}$ stretching of amide groups. The intense superposed peaks between $1200-900 \mathrm{~cm}^{-1}$ correspond to several bond vibrations (C-O-C, $\mathrm{C}-\mathrm{O}$ and $\mathrm{C}-\mathrm{O}-\mathrm{H}$ stretching, $\mathrm{N}-\mathrm{H}$ bending, and $\mathrm{O}-\mathrm{H}$ and $\mathrm{C}=\mathrm{O}$ deformation). 
The PEA spectrum shows a broad absorbance band centred at $3400 \mathrm{~cm}^{-1}$ with low intensity attributed to $\mathrm{N}-\mathrm{H}$ groups of the crosslinking agent, MBAA, and $\mathrm{O}-\mathrm{H}$ stretching of bound water. The overlapping of multiple peaks between 3000 and $2800 \mathrm{~cm}^{-1}$ is associated with the $\mathrm{C}-\mathrm{H}$ stretching in sp3-hybridized carbons. The intense peak at $1735 \mathrm{~cm}^{-1}$ is attributed to free $\mathrm{C}=\mathrm{O}$ stretching of ester groups and a lower peak at $1660 \mathrm{~cm}^{-1}$ is associated to the carbonyls of the MBBA (Amide I). Multiple bands in the region from 1000 to $1200 \mathrm{~cm}^{-1}$ are attributed to $\mathrm{C}-\mathrm{O}-\mathrm{H}$ and C-O stretching.

The different intermediate compositions of poly(MeHA-co-EA) copolymer networks show characteristic bands belonging to groups of the homopolymer networks. Therefore, larger amounts of MeHA in the copolymer networks resulted in changes in the spectra, such as a more intense band between $3000 \mathrm{~cm}^{-1}$ and $2800 \mathrm{~cm}^{-1}$, between $1600 \mathrm{~cm}^{-1}$ and $1500 \mathrm{~cm}^{-1}$ and the peaks around $1400 \mathrm{~cm}^{-1}$ and $1000 \mathrm{~cm}^{-1}$. Moreover, the increase of MeHA in the copolymer networks provokes a major overlapping of different bands and peaks. Moreover, as can be observed in Figure 3. 37, the intense peak at $1735 \mathrm{~cm}^{-1}$ corresponding to the $\mathrm{C}=\mathrm{O}$ stretching and the overlapped bands centred at $1600 \mathrm{~cm}^{-1}$ are attributed to carboxylates, Amide I and Amide II remain independent for all the intermediated compositions studied. However, bands at $1660 \mathrm{~cm}^{-1}$ attributed to the carbonyl stretching of the MBBA are overlapped with the ones at $1600 \mathrm{~cm}^{-1}$ corresponding to the Amide I of the MeHA component.

\subsubsection{Swelling experiments}

In order to determine the influence of the nominal PEA weight fraction (WEA) on swelling, the EWC of the different poly(MeHA-co-EA) copolymer networks and PEA was evaluated (see section 2.3). The EWC was studied on samples after 3 days immersed in $0.15 \mathrm{M}$ PBS with the aim to mimic the swelling in physiological conditions and shield electrostatic interactions because of the anionic nature of $\mathrm{HA}$ and hence MeHA. 


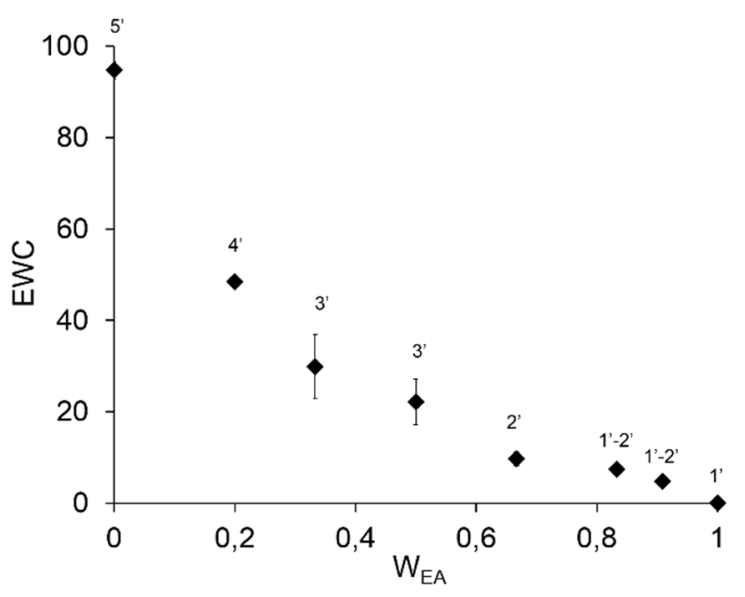

Figure 3. 38. The equilibrium water content of the different poly(MeHA-co-EA) copolymer networks as function of $\mathrm{W}_{\mathrm{EA} \text {. }}$

The Figure 3. 38 shows that the EWC value significantly decreases for higher weight fractions of EA. The MeHA homopolymer resulted in a value of EWC of $94.7 \pm 1.8$, and small increments in the $E A$ content $\left(\mathrm{W}_{\mathrm{EA}}=0.2\right)$ halve such value $(49.4 \pm 1.2)$. Moreover, greater $W_{E A}$ produces a continuous reduction of the EWC value. However, the reduction of the EWC values turns stable in samples with $W_{E A}$ beyond 0.7 until reaching values of $4.8 \pm 0.5$ in samples with the higher content of EA. The PEA homopolymer resulted in a value of $0.07 \pm 0.02$.

\subsubsection{Compression tests}

In order to determine the influence of the EA to MeHA weight ratio on the mechanical properties of the copolymer networks, the samples were studied under unconfined compression. The compression was conducted on the different samples at equilibrium swelling in $0.15 \mathrm{M}$ PBS. From the compression tests, the stress-strain curves were determined employing Equation 2. 9. Additionally, the stress-strain curves were used to calculate the mechanical parameters of the different copolymer networks: initial modulus, $E_{0}$, final modulus, $E_{f}$, and final strain, $\varepsilon_{f}$. 


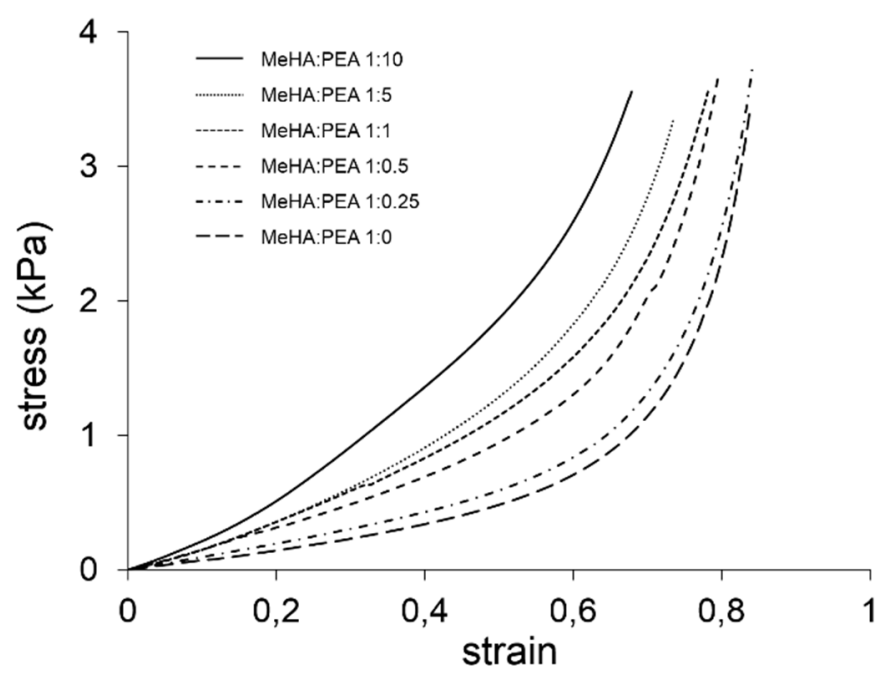

Figure 3. 39. Stress-strain curves of the different poly(MeHA-co-EA) copolymer networks. The study was conducted with samples swollen in $0.15 \mathrm{M}$ PBS to equilibrium.

Figure 3. 39 shows the mechanical behaviour of the different compositions of poly(MeHA-co-EA) copolymer networks. In the figure, it can be observed that all the compositions had a similar mechanical behaviour under compression, and the stress-strain curves had a similar trend: an initial linear stage and, after a small transition region, the curves display a vertical asymptotic behaviour at advanced compression. However, significant differences were found when changing the composition: samples with higher amounts of MeHA widen the linear region and, hence the vertical asymptote was shown at greater strains. 

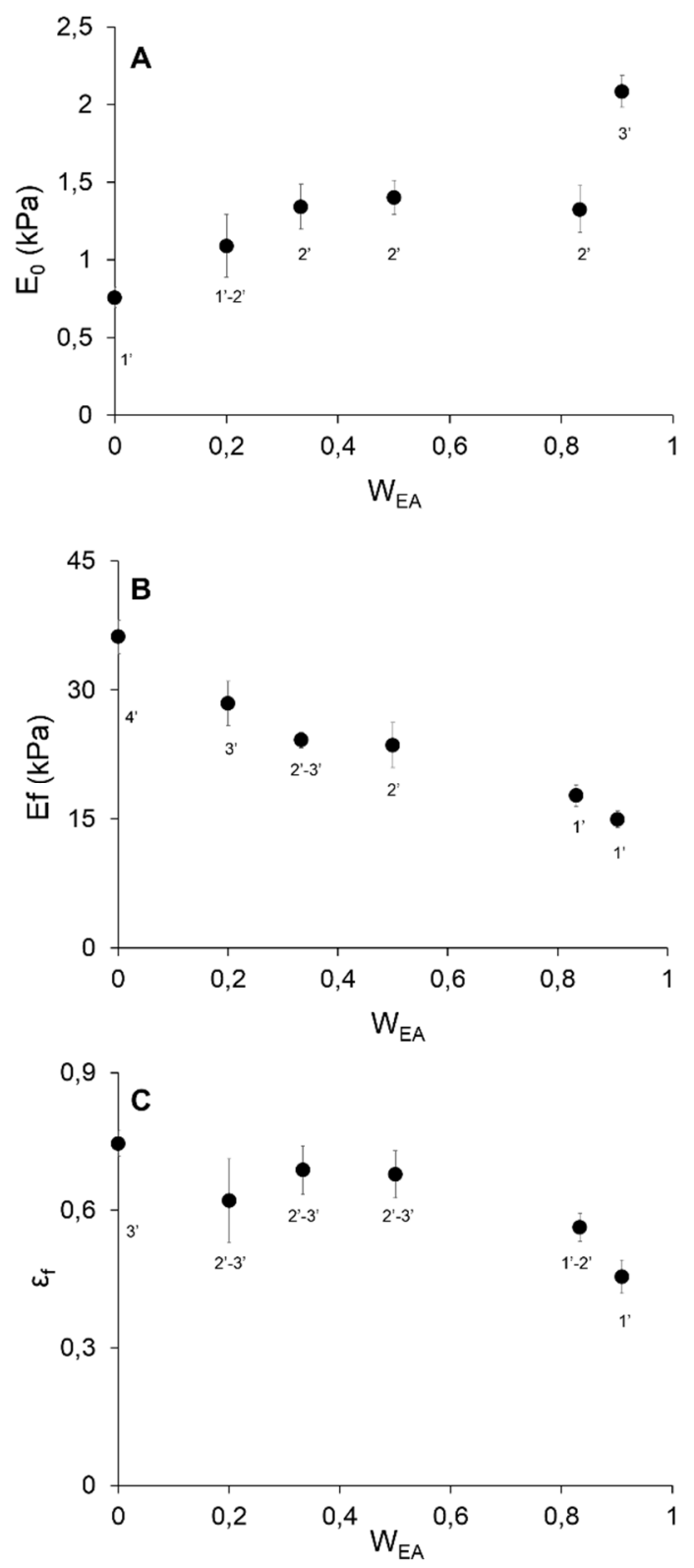

Figure 3. 40. Parameters of the poly(MeHA-co-EA) copolymer networks obtained from the stressstrain curves: A) Initial modulus, B) final modulus and C) final strain. 
Figure 3.40 shows the parameters determined from the stress-strain curves from the compositions plotted in Figure 3. 39. The determined parameters showed differences depending on the nominal PEA, weight ratio of EA to MeHA in the copolymer networks.

The $E_{0}$ value (Figure 3. $40 \mathrm{~A}$ ) was found to be in the range between 0.5 and 2.5 $\mathrm{kPa}$ in the different samples. This modulus increased faster its value from $0.7 \mathrm{kPa}$ in samples with EA weight fractions of 0.5 until reaching values of $1.4 \mathrm{kPa}$ in samples with $E A$ weight fractions of 0.3 . Moreover, this value of $E_{0}$ became stable in samples with higher content of EA. However, the value of $E_{0}$ increases significantly with respect to each other composition $(2.1 \mathrm{kPa})$ in the copolymer network with the highest content of EA.

The $E_{f}$ value (Figure 3. $40 \mathrm{~B}$ ), which is related with the strain when the vertical asymptote is reached, shows a different trend between the different samples than for the $E_{0}$ values. Moreover, $E_{f}$ had relative higher values than $E_{0}$ and resulted to be in the range from 15 to $37 \mathrm{kPa}$. Therefore, the trend of the $E_{f}$ showed decreasing values in compositions with higher EA weight fractions. However, in samples with the highest weight fraction of EA, the $E_{f}$ value seemed to reach a stable behaviour.

The $\varepsilon_{f}$ values (Figure 3. $40 \mathrm{C}$ ) in the different copolymer networks resulted to have decreasing values (from 0.75 to 0.5 ) with respect to the EA weight fraction. Therefore, the $\varepsilon_{f}$ value was significantly reduced in samples with higher amounts of EA, (WEA between 0.83 and 0.91 ). Therefore, the samples with larger amounts of EA reached lower strains under the load range studied.

\subsubsection{Morphological characterization}

In order to determine the macroscopic integration of both components within the poly(MeHA-co-EA) copolymer networks and the structural changes between compositions, the inner morphology of different compositions was analysed by scanning electron microscopy (section 2.3). 

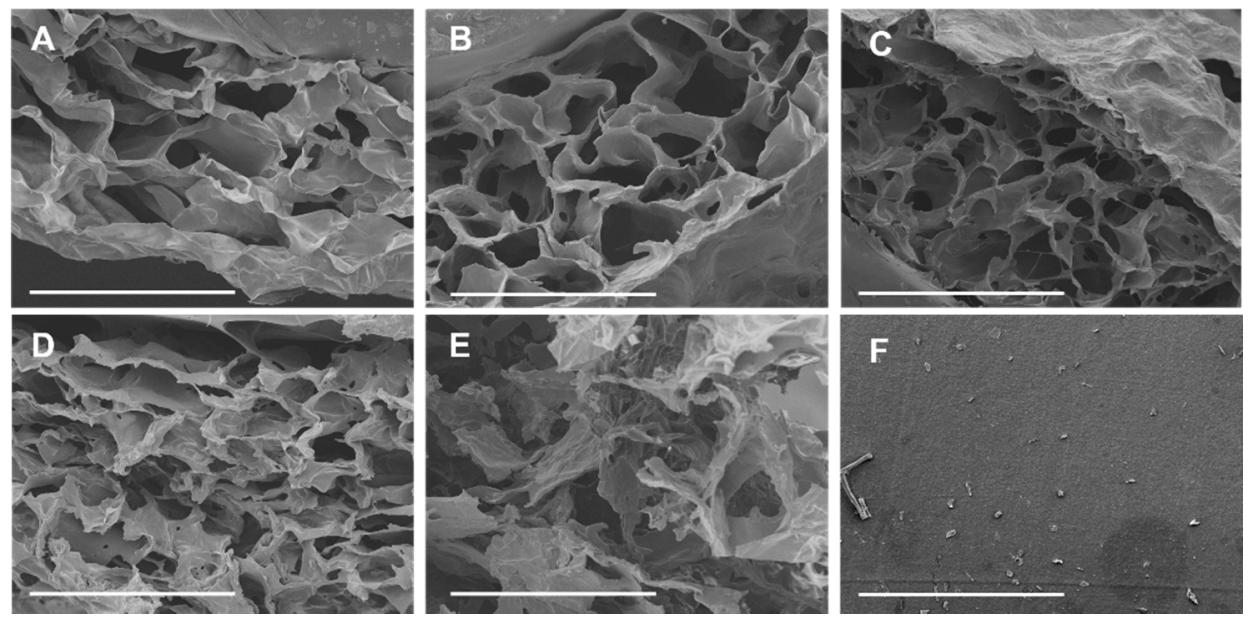

Figure 3. 41. Cross-section of MeHA:PEA 1:0 (A), MeHA:PEA 1:0.5 (B), MeHA:PEA 1:1 (C), MeHA:PEA 1:5 (D), MeHA:PEA 1:10 (E) copolymer networks, and PEA homopolymer (F). Scale bar $600 \mu \mathrm{m}$.

Figure 3. 41 displays the inner structure in the different copolymer networks and PEA homopolymer network. The MeHA:PEA 1:0 sample showed a porous structure with a sheet-like shape. The PEA homopolymer network shows a flat non-porous structure. The different copolymer networks show continuous porous flat structures with circular porous with different sizes. However, this structure becomes more closed with increasing $W_{\text {EA. }}$ Therefore, the MeHA:PEA 1:10 sample showed a closed and unstructured inner matrix.

\subsubsection{Thermogravimetric analysis}

In order to determine the thermal stability and degradation stages, the different poly(MeHA-co-EA) copolymer networks were analysed by thermogravimetry from room temperature to $700{ }^{\circ} \mathrm{C}$ (see section 2.3). Moreover, the thermal degradation of the intermediate copolymers were compared with the MeHA:PEA 1:0 and PEA samples with the aim of investigating some change in thermal behaviour due to the incorporation of both polymers. From the thermogravimetric analyses, the corresponding thermograms of remaining weight and derivative weight loss as functions of the temperature were obtained. Additionally, thermograms were employed to calculate some thermal parameters including maximum rate of weight loss in each weight loss stage, weight remaining after each stage and residual content at $700 \stackrel{\circ}{\circ}$. 
A

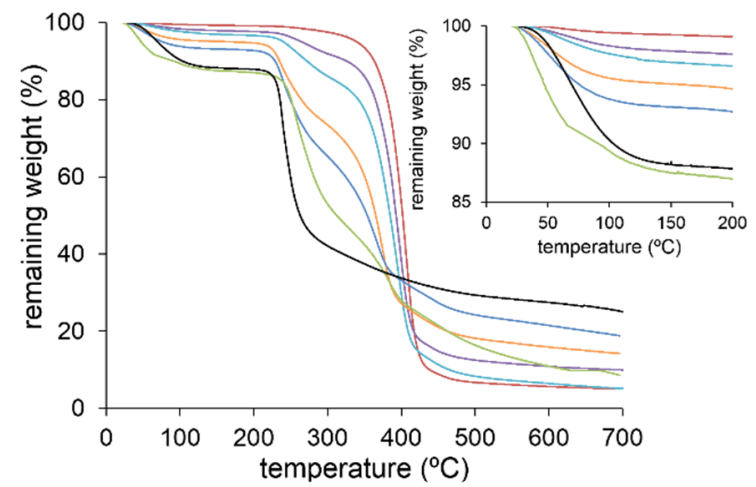

B

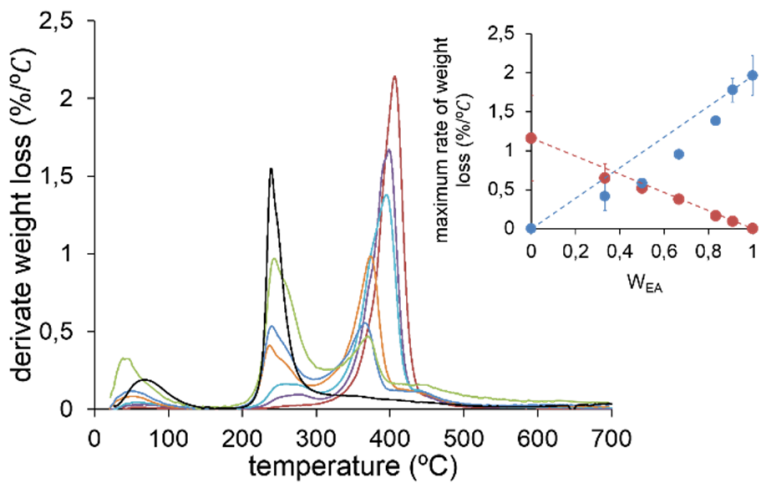

Figure 3. 42. Remaining weight $(A)$ and derivative weight loss $(B)$ thermograms of the different poly(MeHA-co-EA) copolymer networks: MeHA:PEA 1:0 (black line), MeHA:PEA 1:0.5 (green line), MeHA:PEA 1:1 (blue line), MeHA:PEA 1:2 (orange line), MeHA:PEA 1:5 (cyan line), MeHA:PEA 1:10 (purple line) and PEA (red line). The inset in "A" shows a detail of the thermograms at low temperatures. The Inset in "B" shows the maximum rate of weight loss between 200 and $300^{\circ} \mathrm{C}$ (red circles) and between $300{ }^{\circ} \mathrm{C}$ and $500 \stackrel{\circ}{\circ}$ (blue circles) as function of $W_{E A}$.

As can be observed in Figure 3. 42, the thermograms of the homopolymers, MeHA:PEA 1:0 and PEA samples, revealed two and one degradation stages, respectively. The thermogram of the MeHA:PEA 1:0 sample showed a first weight loss step from room temperature to $180{ }^{\circ} \mathrm{C}$ and a second one from $200{ }^{\circ} \mathrm{C}$ until the final temperature recorded. The thermogram of the PEA homopolymer network contains a unique degradation stage from $220 \stackrel{\circ}{\circ} \mathrm{C}$ to $500 \stackrel{\circ}{\circ} \mathrm{C}$. The thermograms of the intermediate poly(MeHA-co-EA) copolymer networks resulted to have three different degradation stages, and each one is located in the temperature range of those corresponding to the homopolymers: the two early weight loss stages were assumed to the MeHA component and the last one to the PEA matrix. However, in Figure 3. 42, it can be observed that the second weight loss stage is overlapped with the third one. On the other hand, the 
homopolymer networks resulted to have a residual content of $23.2 \%$ in the MeHA:PEA 1:0 sample and $3.1 \%$ in PEA homopolymer, whereas the intermediate copolymer networks showed residual contents with values between those corresponding to the homopolymer networks (see Table 3. 10).

At the first weight loss stage, in samples containing MeHA, the different materials show similar trends. However, in general, the lower the amounts of MeHA in the samples, the lower the maximum rate of weight loss and weight loss at the end of such stage (see Table 3. 10). Moreover, the maximum rate of weight loss in the intermediate compositions $\left(46^{\circ} \mathrm{C}\right.$ ) is reached at earlier temperatures than that of the MeHA homopolymer network ( $72^{\circ} \mathrm{C}$ ).

In the second stage of degradation, in the samples containing MeHA, it can be observed as the weight loss is higher in the MeHA:PEA 1:0 samples and it becomes lower in samples with larger amounts of EA. Moreover, the maximum degradation rate appears at similar temperature for all the mentioned samples, but the higher the amounts of MeHA, the lower the maximum weight loss rate (see Table 3. 10). The PEA homopolymer network did not reveal any degradation stage corresponding to this temperature range.

The third degradation stage was found in samples containing PEA. In this case, decreasing the EA content in the poly(MeHA-co-EA) copolymer networks resulted in a relative lower weight loss in this stage. PEA homopolymer networks resulted to have a higher maximum degradation rate than the intermediate compositions (see Table 3. 10). Moreover, larger amounts of MeHA in the samples resulted in lower degradation rates. In the case of poly(MeHA-co-EA) copolymer networks, such maximum was displaced to lower temperatures with increasing the MeHA content in the samples. 
Table 3. 10. Parameters determined from the TGA thermograms of different poly(MeHA-co-EA) copolymer networks.

\begin{tabular}{ccccc}
\hline $\begin{array}{c}\text { Copolymer } \\
\text { network } \\
\text { composition }\end{array}$ & $\begin{array}{c}\text { Remaining } \\
\text { weight after } \\
\text { the first } \\
\text { stage (\%) }\end{array}$ & $\begin{array}{c}\text { Maximum rate of } \\
\text { weight loss in } \\
\text { the second stage }\end{array}$ & $\begin{array}{c}\text { Maximum rate } \\
\text { of weight loss } \\
\text { in the third } \\
\text { stage }\end{array}$ & $\begin{array}{c}\text { Residual } \\
\text { content } \\
\text { (\%) }\end{array}$ \\
\hline MeHA:PEA 1:0 & 86.9 & 1.2 & 0.0 & 23.2 \\
MeHA:PEA 1:1 & 92.1 & 0.5 & 0.6 & 17.9 \\
MeHA:PEA 1:2 & 94.8 & 0.4 & 1.0 & 14.6 \\
MeHA:PEA 1:5 & 97.7 & 0.2 & 1.4 & 5.2 \\
MeHA:PEA 1:10 & 98.9 & 0.1 & 1.8 & 10.2 \\
PEA & 99.7 & ---- & 2.0 & 3.1 \\
\hline
\end{tabular}

\subsubsection{Differential scanning calorimetry}

In order to determine thermal transitions in the different poly(MeHA-co-EA) copolymer networks, different compositions of such copolymers and the homopolymer networks were analysed by differential scanning calorimetry (DSC). The analysis was conducted in the temperature range between $-60{ }^{\circ} \mathrm{C}$ and $30{ }^{\circ} \mathrm{C}$ and was performed for samples after the freeze-drying process and after an additional drying (see section 2.3) with the aim of removing possible bound water. The analysis of homopolymer and copolymer networks was performed with the aim of observing some changes in thermal transitions due to the incorporation of both components. From the DSC analyses, the corresponding thermograms, from the normalization of the recorded heat flow by the mass of the sample and the rate of heating, were obtained. Additionally, thermograms were employed to determine the glass transition temperature by the inflexion point method (Table 3. 11). 

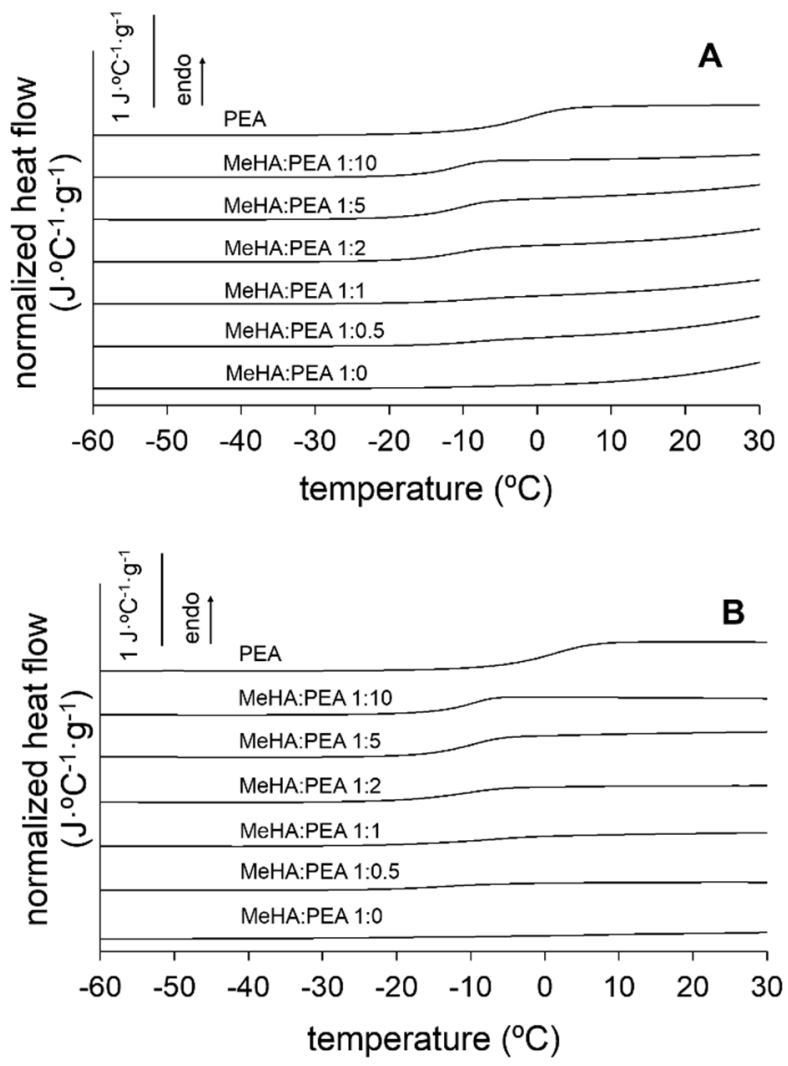

Figure 3. 43. DSC scans of the different poly(MeHA-co-EA) copolymer networks after the freezedrying $(A)$ and an additional drying step (B).

The DSC thermograms after the freeze-drying process (Figure 3. 43-A) and after additional drying (Figure 3. 43-B) showed analogous trends. The thermograms of each composition show a main transition corresponding to the glass transition, in samples after the freeze-drying process $\left(\mathrm{Tg}_{1}\right)$ and after the additional drying $\left(\mathrm{Tg}_{2}\right)$ (see Table 3. 11).

Figure 3. 43-A shows the DSC thermograms of the homopolymer networks (MeHA:PEA 1:0 and PEA) and different compositions of the poly(MeHA-co-EA) copolymer networks after the freeze-drying process. The vitreous transition $\left(\mathrm{Tg}_{1}\right)$ in the PEA homopolymer network and in the different poly(MeHA:PEA) copolymer networks was displayed at different temperatures. Therefore, PEA showed the glass transition around $-1.5^{\circ} \mathrm{C}$, while those in the different copolymer networks was displaced to lower temperatures, around $-10^{\circ} \mathrm{C}$, (see Table 3. 12). Moreover, among the different copolymer networks, the glass transition was observed at similar temperatures, but its amplitude was reduced with increasing the MeHA content. In the case of MeHA:PEA 1:0 sample the glass transition was not 
observed. In the thermograms of samples containing MeHA, a change in the heat flow was observed at $5{ }^{\circ} \mathrm{C}$ until the final temperature recorded. This phenomenon yields an endothermic change and its intensity is more significant in samples with higher amounts of MeHA.

Figure 3. 43-B shows the thermograms of the different samples after the additional drying process. In this case, the endothermic change from $5{ }^{\circ} \mathrm{C}$ to 30 ${ }^{\circ} \mathrm{C}$ in samples containing MeHA was not observed. On the other hand, the glass transition process $\left(\mathrm{Tg}_{2}\right)$ occurs at similar temperatures than in the series without additional drying process. Therefore, PEA homopolymers had the glass transition process at $1.2^{\circ} \mathrm{C}$, while the copolymer networks show a displacement to lower temperatures (around $-10^{\circ} \mathrm{C}$ ). However, the increment of MeHA content yields to a reduction in the change of the heat flow intensity through the glass transition.

Considering that PEA constitutes a completely amorphous phase in all the compositions, the experimental PEA content in them was estimated from the change in the normalized heat flow through the glass transition. Therefore, the experimental PEA fraction in each copolymer network was determined from the ratio between the change in the normalized heat flow of each composition and the one of the PEA homopolymer (Table 3. 11). The experimental PEA content was also obtained from the thermograms of the samples after the freeze-drying process (WPEA1) and the ones after the additional drying process (WPEA2). The experimental PEA content determined from both sample groups was compared with the nominal one, referred as the EA weight fraction (WEA) employed in the copolymerization reaction.

Table 3. 11. Temperature of glass transition and PEA weight fraction in the copolymer networks.

\begin{tabular}{cccccccc}
\hline $\mathbf{W}_{\text {EA }}$ & $\mathbf{0}$ & $\mathbf{0 . 3}$ & $\mathbf{0 . 5}$ & $\mathbf{0 . 7}$ & $\mathbf{0 . 8}$ & $\mathbf{0 . 9}$ & $\mathbf{1}$ \\
\hline $\mathrm{Tg}_{1}\left({ }^{\circ} \mathrm{C}\right)$ & --- & -8.91 & -9.06 & -10.73 & -10.19 & -10.79 & -1.46 \\
$\mathrm{Tg}_{2}\left({ }^{\circ} \mathrm{C}\right)$ & --- & -12.56 & -7.72 & -10.39 & -9.68 & -9.69 & 1.22 \\
$\mathrm{~W}_{\text {PEA1 }}$ & 0.00 & 0.33 & 0.30 & 0.43 & 0.61 & 0.81 & 1.00 \\
$\mathrm{~W}_{\mathrm{PEA} 2}$ & 0.00 & 0.23 & 0.24 & 0.48 & 0.60 & 0.86 & 1.00 \\
\hline
\end{tabular}




\subsubsection{Dynamic mechanical thermal analysis}

Dynamic mechanical thermal analysis (DMTA) of the different homopolymer and copolymer networks were performed with the aim of evaluating variations in the mechanical properties in the temperature range from $-60{ }^{\circ} \mathrm{C}$ to $60{ }^{\circ} \mathrm{C}$ (see section 2.3). Therefore, those changes in mechanical properties are related with transitions in the motion of the polymer chains of both components due to temperature variations. These differences were measured by DMTA recording the variation of the storage and loss modulus ( $E^{\prime}$ and $E^{\prime \prime}$, respectively) as function of the temperature. Moreover, the ratio between $E^{\prime \prime}$ and $E^{\prime}(\tan \delta)$, which constitutes a loss factor, was employed as an estimation of the viscoelastic behaviour.
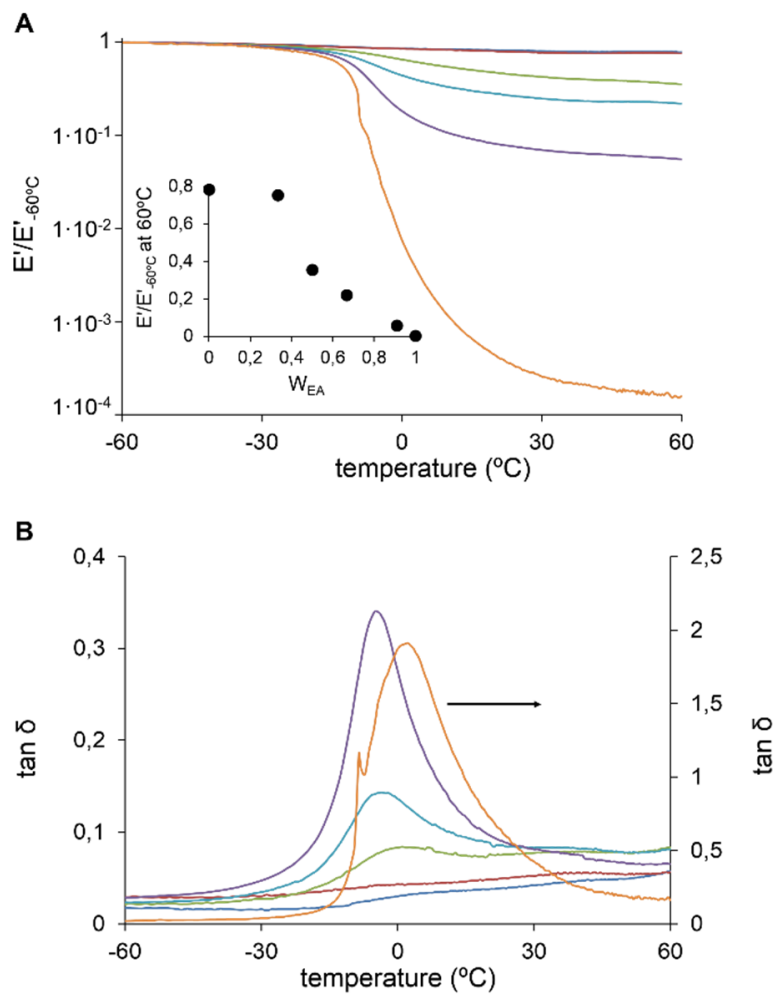

Figure 3. 44. Dynamic-mechanical properties of the different poly(MeHA-co-EA) copolymer networks: MeHA:PEA 1:0 (blue line), MeHA:PEA 1:1 (red line), MeHA:PEA 1:2 (green line), MeHA:PEA 1:5 (cyan line), MeHA:PEA 1:10 (purple line), and PEA (orange line). (A) Thermograms of $E^{\prime} / E_{-60}^{\prime}{ }^{\circ} \mathrm{C}$. (B) Thermograms of the loss tangent, $\tan \delta$, the arrow indicates the axis for PEA homopolymer network. Inset in "A" shows the value of $E^{\prime} / E_{-60^{\circ} \mathrm{C}}$ at $60{ }^{\circ} \mathrm{C}$ as function of the $\mathrm{W}_{\text {EAA. }}$. 
Figure 3. 44-A shows the variation in the storage modulus as function of the temperature for different copolymers and both homopolymer networks. In general, the samples show the principal mechanical-dynamical relaxation from $30 \stackrel{\circ}{ } \mathrm{C}$ to $50 \stackrel{\circ}{ } \mathrm{C}$ increasing its amplitude for samples with higher weight fractions of EA. However, this transition was not observed for MeHA:PEA 1:0 and MeHA:PEA 1:1 samples. Therefore, this transition was attributed to the glass transition of the PEA homopolymer network. At temperatures below the principal transition $(-30 \stackrel{\circ}{\circ})$, the PEA homopolymer network shows the highest storage modulus value (around $2 \times 10^{9} \mathrm{~Pa}$ ), whereas this modulus for copolymer and MeHA homopolymer networks resulted in much lower values (between $1 \times 10^{7}$ and $\left.2 \times 10^{8}\right)$. Moreover, this value of modulus decreased for copolymer networks with lower amounts of EA. In the region of temperatures above the transition, the storage modulus values evidenced a different behaviour. In this case, the PEA homopolymer network resulted in lower modulus values (around $4 \times 10^{5} \mathrm{~Pa}$ ), while the copolymer and the MeHA homopolymer networks displayed slightly higher values (between $9 \times 10^{6}$ and $1.6 \times 10^{7} \mathrm{~Pa}$ ). Taking into account the representation of Figure 3. 44 where the storage modulus is showed as the ratio between $E^{\prime}$ at each temperature and E' at $-60^{\circ}$, a reduction in such ratio can be appreciated for samples containing larger amounts of EA at temperatures above the main transition. This observation is clearly displayed in the inset of Figure 3. 44.

Figure 3. 44-B shows the loss tangent, $\tan \delta$, as function of the temperature. The PEA homopolymer network resulted to show the highest loss tangent value (2.8) and copolymer networks showed much lower values (from 0.34 to 0.08), decreasing such value in samples with a lower weight fraction of EA. Despite the observed changes in the loss tangent of the MeHA:PEA 1:0 and MeHA:PEA 1:1 samples, their respective maximums were not well defined and were not taken into account. The maximum of the loss tangent is manifested at $2.2^{\circ} \mathrm{C}$, whereas the corresponding ones of the copolymer networks was displaced to lower temperatures (between -1 and $-5 \stackrel{\circ}{\circ}$ ); however, the higher the EA weight fraction, the lower the temperature at which appeared the maximum. 


\subsection{Enzymatic degradation}

In order to somehow predict their behaviour in physiological conditions, enzymatic degradation of the different HA-based materials (solv_HA:DVS 1:0.9 samples, HA-DVS scaffolds and poly(MeHA-co-EA) copolymer networks) was carried out in buffer medium consisting in $0.15 \mathrm{M}$ PBS containing 10 units $/ \mathrm{mL}$ of hyaluronidase at $37^{\circ} \mathrm{C}$ (see section 2.3). The employed hyaluronidase is a mammal endo- $\beta-\mathrm{N}$-acetylhexosaminidase $(\mathrm{PH}-20)$ which possesses hydrolase activity, yielding as final products small oligosaccharides. The biodegradation of the HA-based materials and its effects were assessed analysing changes in some parameters at different degradation times: weight loss, equilibrium water content, morphology of degraded samples and molecular weight distribution of byproducts.

\subsubsection{Determination of weight loss}

The weight loss of the different samples after the enzymatic degradation was evaluated by comparing the remaining weight at different time points (up to 56 days) with the initial one. In this study, the effect of enzymatic digestion on the weight loss of HA-DVS scaffolds and poly(MeHA-co-EA) copolymer networks was evaluated separately. The degradation of the solv_HA:DVS 1:0.9 samples and HA-DVS scaffolds was compared. In order to determine some hydrolysis due to the effect of $0.15 \mathrm{M}$ PBS, samples swollen at equilibrium for 3 and 56 days were taken as controls.

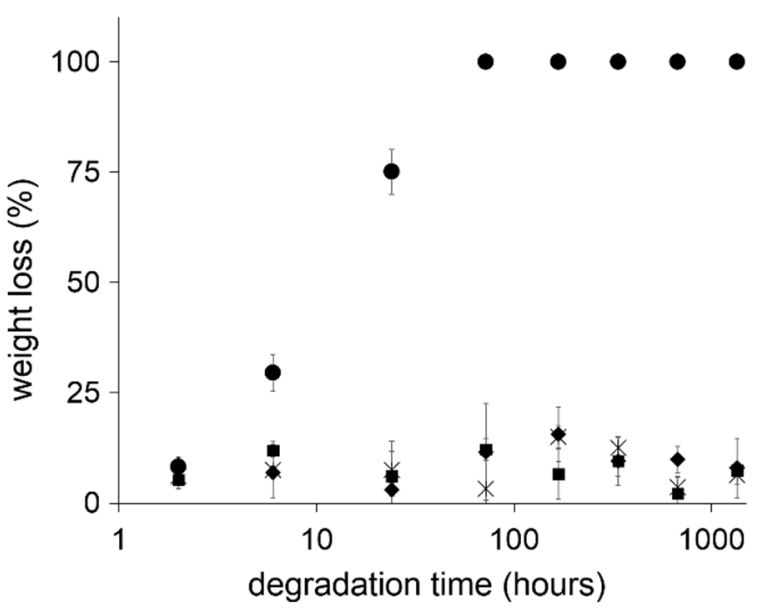

Figure 3. 45. Percentage of weight loss of solv_HA:DVS 1:0.9 (circles) and HA-DVS scaffolds: scaff_HA:DVS 1:0.6 (asterisks), scaff_HA:DVS 1:0.9 (squares) and scaff_HA:DVS 1:1.5 (diamonds), at different degradation times. 


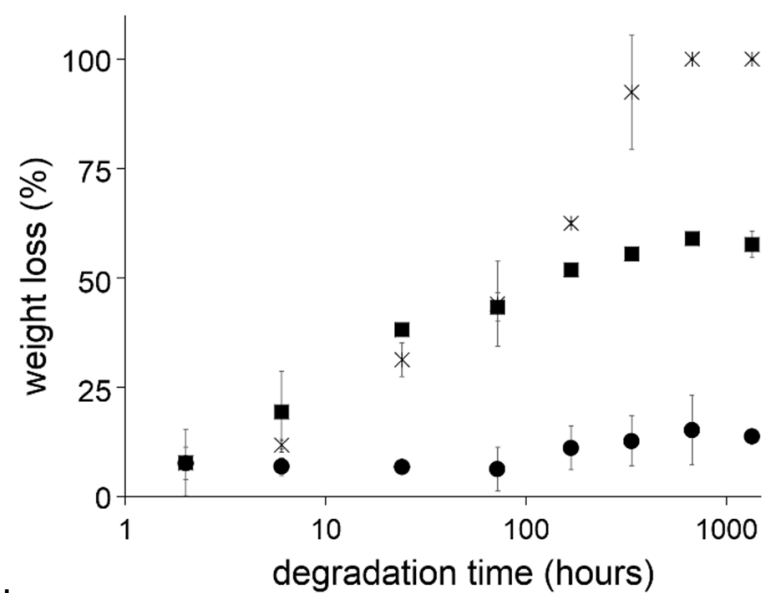

Figure 3. 46. Weight loss at different degradation times of poly(MeHA-co-EA) polymer networks: MeHA:PEA 1:0 (asterisks), MeHA:PEA 1:1 (squares) and MeHA:PEA 1:10 (circles).

Figure 3. 45 and Figure 3. 46 display significant differences in terms of the effect of hyaluronidase in the weight loss depending on the type of sample. Therefore, the solv_HA:DVS 1:0.9 materials show the highest digestion rates yielding to a complete degradation after 3 days. Moreover, the degradation of these samples can be clearly observed after 6 hours in the enzymatic solution. The different samples immersed in 0.15 M PBS for 3 and 56 days showed around $2 \%$ of weight loss.

The exposition of the different HA-DVS scaffolds to hyaluronidase (Figure 3. 45) resulted in a non-significant effect in terms of their weight loss along the studied period (in most cases weight loss resulted below $15 \%$ ). Moreover, the weight losses of these materials subjected to the degradation process and controls (samples immersed in $0.15 \mathrm{M}$ PBS) resulted in similar values (in all cases the weight loss was below $15 \%$ ).

The enzymatic degradation of poly(MeHA-co-EA) copolymer networks (Figure 3. 46) was found to depend on the EA weight fraction employed in the copolymerization reaction. The MeHA:PEA 1:0 samples suffer complete weight loss between 14 and 28 days and the degradation is observed after one day in the enzymatic solution. However, the MeHA:PEA 1:1 and MeHA:PEA 1:10 samples are only partially digested, reaching weight losses below $60 \%$ and 15 $\%$, respectively, after 52 days of enzymatic degradation. Moreover, the weight loss of the poly(MeHA-co-EA) copolymer networks in $0.15 \mathrm{M}$ PBS in equilibrium at 3 and 56 days represents values below $7 \%$. 


\subsubsection{Determination of equilibrium water content}

In order to evaluate the effect of enzymatic degradation on swelling, the equilibrium water content, EWC, of the different HA-based materials was evaluated after the enzymatic degradation. The swelling in a solution of $0.15 \mathrm{M}$ PBS is employed with the aim to shield electrostatic repulsions due to HA moieties. Additionally, the EWC value at different times was compared with that of controls after 3 and 56 days in 0.15 M PBS.

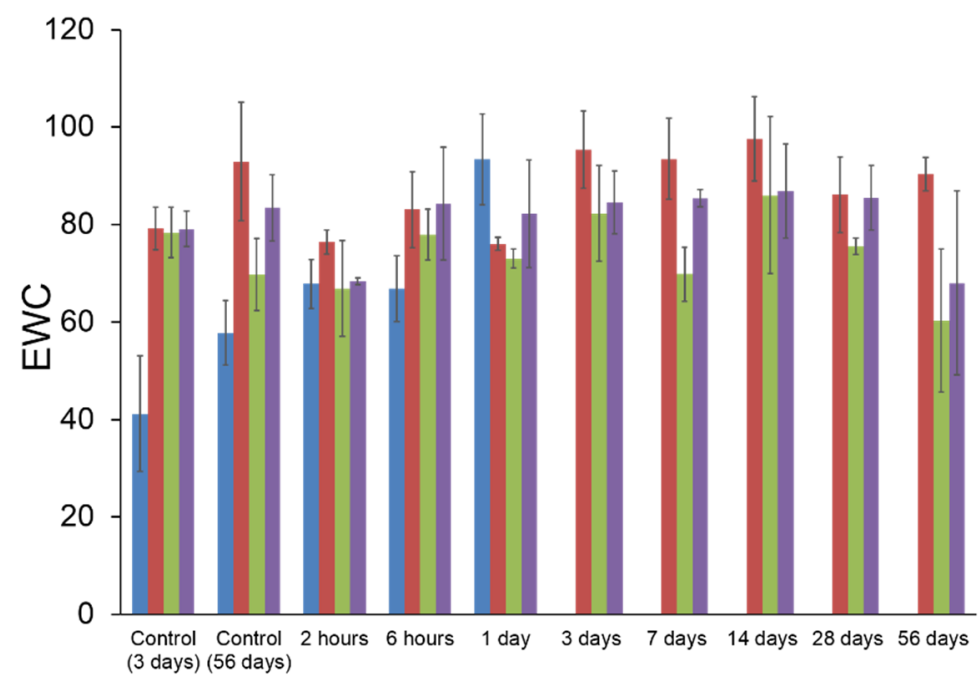

Figure 3. 47. Equilibrium water content of solv_HA:DVS 1:0.9 (blue bars) and HA-DVS scaffolds: scaff_HA:DVS 1:0.6 (red bars), scaff_HA:DVS 1:0.9 (green bars) and scaff_HA:DVS 1:1.5 (purple bars), immersed in $0.15 \mathrm{M}$ PBS at different degradation times. The two first groups represent samples immersed in $0.15 \mathrm{M}$ PBS without enzyme (time is shown in brackets). Vertical bars show the standard deviation. 


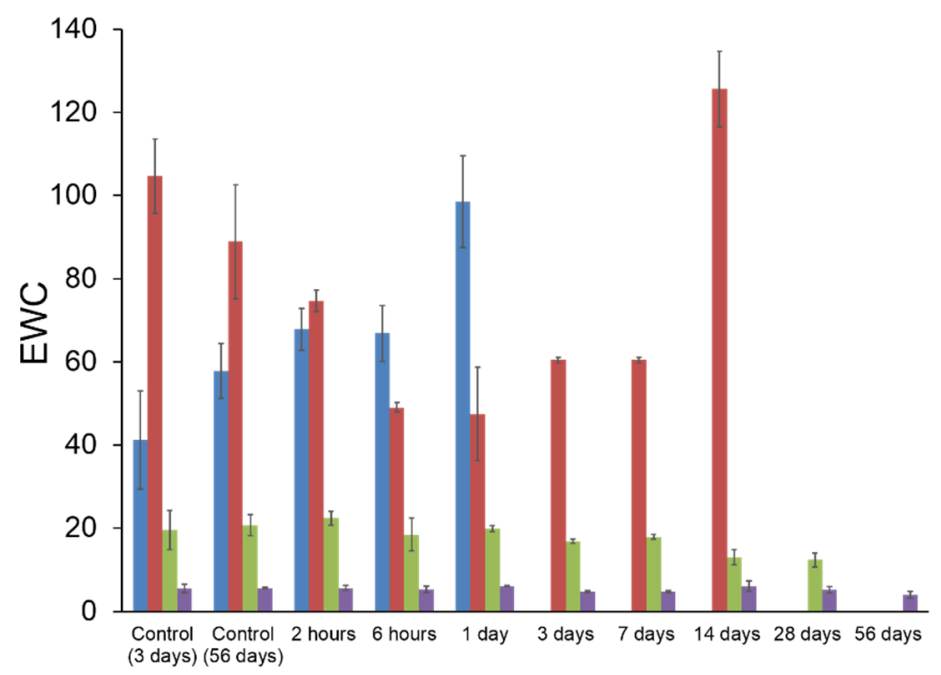

Figure 3. 48. Equilibrium water content of solv_HA:DVS 1:0.9 (blue bars) and poly(MeHA-co-EA) copolymer networks: MeHA:PEA 1:0 (red bars), MeHA:PEA 1:1 (green bars) and MeHA:PEA 1:10

(purple bars), immersed in $0.15 \mathrm{M}$ PBS at different degradation times. The two first groups represent samples immersed in 0.15 M PBS without enzyme (time is shown in brackets). Vertical bars show the standard deviation.

Figure 3. 47 and Figure 3. 48 show the variation of the EWC value in the different samples after different times of enzymatic digestion. After the degradation time, each sample was freeze-dried and swollen in $0.15 \mathrm{M}$ PBS for 3 days, taking samples non-exposed to the enzyme and immersed in 0.15 M PBS for 3 and 56 days as controls. In Figure 3. 47, the effect of degradation on the EWC values in the different HA-DVS scaffolds and the solv_HA:DVS 1:0.9 sample was compared. In the meanwhile, In Figure 3. 48, the effect of degradation on the $E W C$ values in the different poly(MeHA-co-PEA) copolymer networks and the solv_HA:DVS 1:0.9 sample was compared

The EWC value of the solv_HA:DVS 1:0.9 samples was increased along the incubation time in the enzymatic solution, ranging between values from 40 to 100 (no significant differences was found between both controls). In the case of HADVS scaffolds, the equilibrium water content value resulted in no variations comparing samples after the different degradation times and controls. Moreover, differences in the water content were not significant between different compositions. The evolution of the EWC value in the different poly(MeHA-co-EA) copolymer networks during enzymatic digestion was found to depend on chemical composition. The MeHA:PEA 1:0 sample showed a reduction in the equilibrium water content from the initial time and after one day incubated with the enzyme; after this time the water content seemed to stabilize between values 
from 50 to 60 and finally the value significantly increased after reaching a value of 125. Differences between both controls resulted to be not significant. The MeHA:PEA 1:1 and MeHA:PEA 1:10 samples resulted in a slight reduction of the equilibrium water content with increasing the degradation time, but differences resulted not significant.

\subsubsection{Morphological characterization}

In order to determine any change in the structure, the vertical sections of the HADVS scaffolds and poly(MeHA-co-EA) copolymer networks were analysed by SEM. The effect of degradation on the different samples was addressed comparing the control materials (at equilibrium swelling for 3 days) with the ones after the enzymatic degradation. Therefore, samples after 56 days in the enzymatic solution were taken as the degraded ones, except for the MeHA:PEA 1:0 sample, in which case the degraded sample was analysed after 14 days in the enzymatic solution because it was completely degraded for longer times (see Figure 3. 45).

In the following figures, the effect of the enzyme on the morphology of the different HA-DVS scaffolds is addressed comparing the internal structure of the scaffolds before the enzymatic digestion (Figure 3. 49) and after 56 days in a solution containing hyaluronidase (Figure 3. 50). 

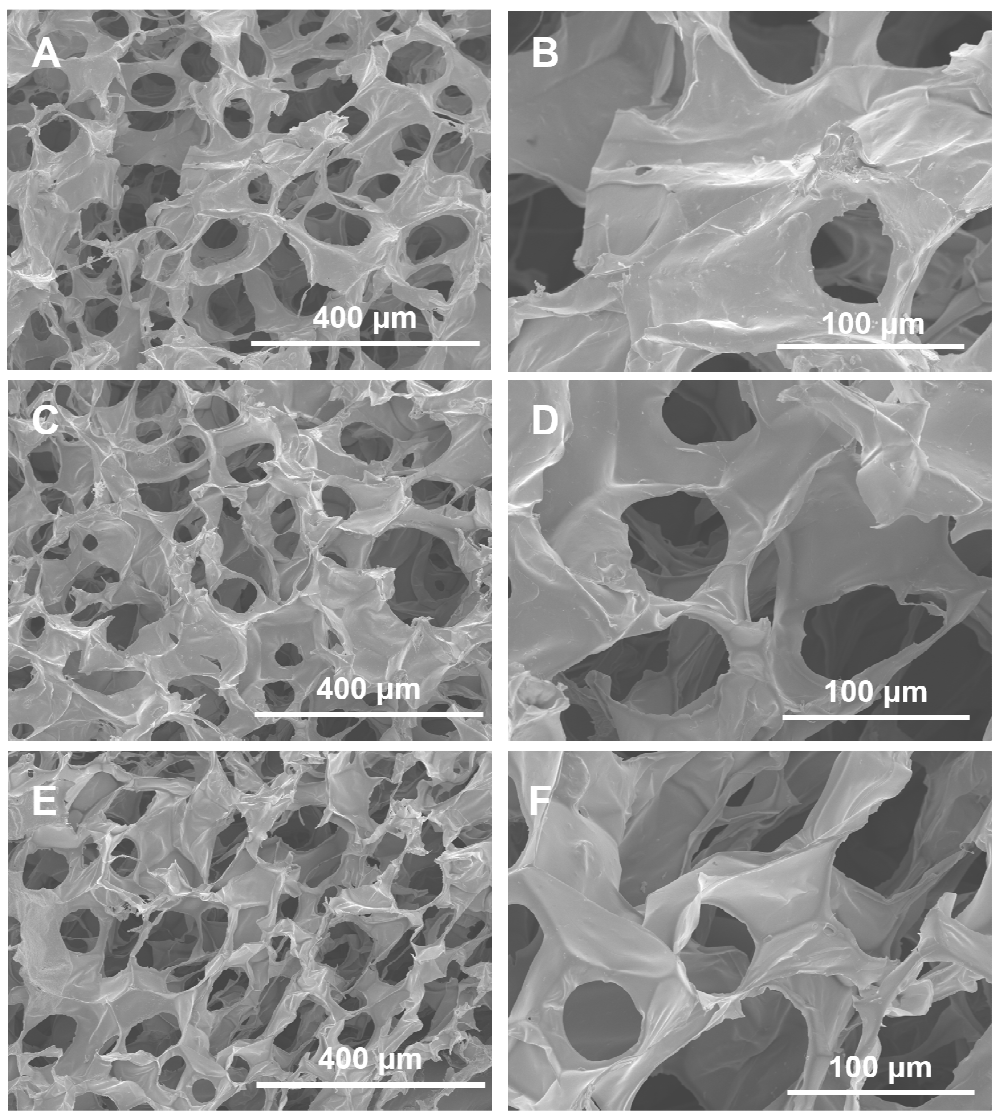

Figure 3. 49. Scanning electron microscopy of the inner structure of the HA-DVS scaffolds: scaff_HA:DVS 1:0.6 (A-B), scaff_HA:DVS 1:0.9 (C-D) and scaff_HA:DVS 1:1.5 (E-F). The images of the samples were taken before the enzymatic solution. 

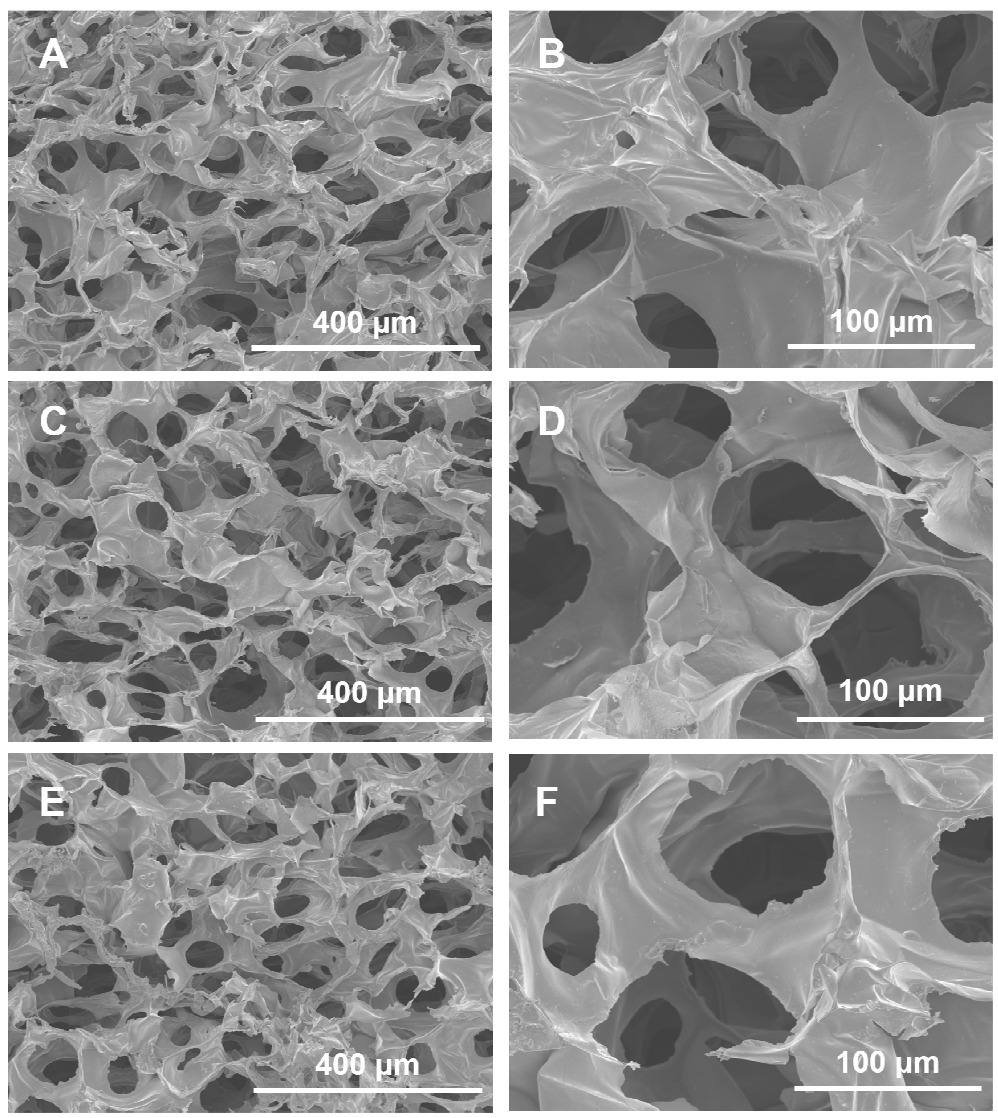

Figure 3. 50. Scanning electron microscopy of the inner structure of the HA-DVS scaffolds (scaff_HA:DVS 1:0.6 (A-B), scaff_HA:DVS 1:0.9 (C-D) and scaff_HA:DVS 1:1.5 (E-F)) immersed for 56 days in the solution containing enzyme.

The internal structure of the HA-DVS scaffolds, before the enzymatic degradation (Figure 3. 49) and after 56 days immersed in the solution containing hyaluronidase (Figure 3. 50) resulted in no evidence of degradation for the different compositions, in terms of morphological changes.

In the following figures, the effect of the enzyme on the morphology of three different poly(MeHA-co-PEA) copolymer networks is determined comparing their internal structure before the enzymatic digestion (Figure 3. 51) and after 56 days in a solution containing hyaluronidase (Figure 3. 52). 

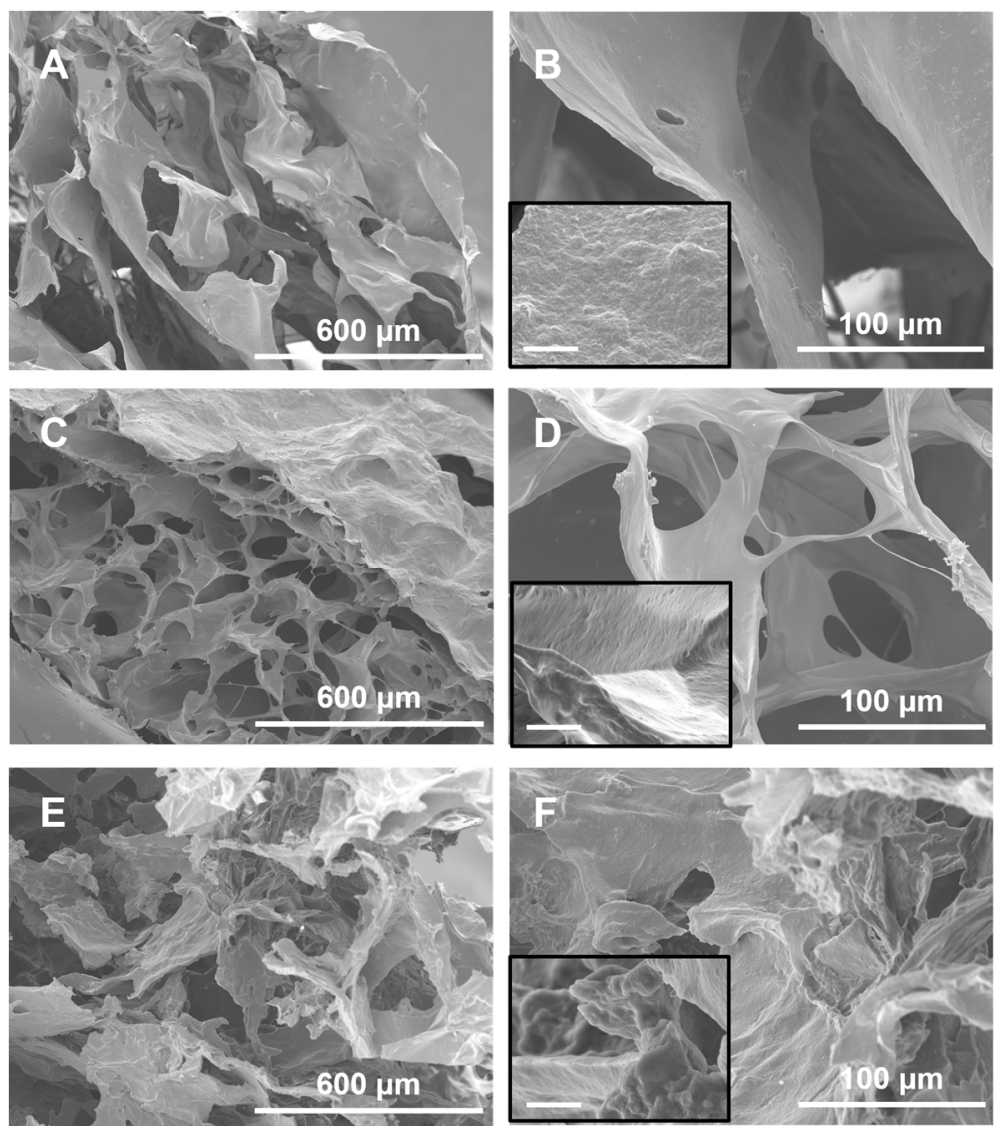

Figure 3. 51. Scanning electron microscopy of the inner structure of the poly(MeHA-co-EA) copolymer networks: MeHA:PEA 1:0 (A-B), MeHA:PEA 1:1 (C-D) and MeHA:PEA 1:10 (E-F). The images of the samples were taken before the enzymatic digestion. Insets show roughness of the inner structures for each composition at high magnification (scale bar $10 \mu \mathrm{m}$ ). 

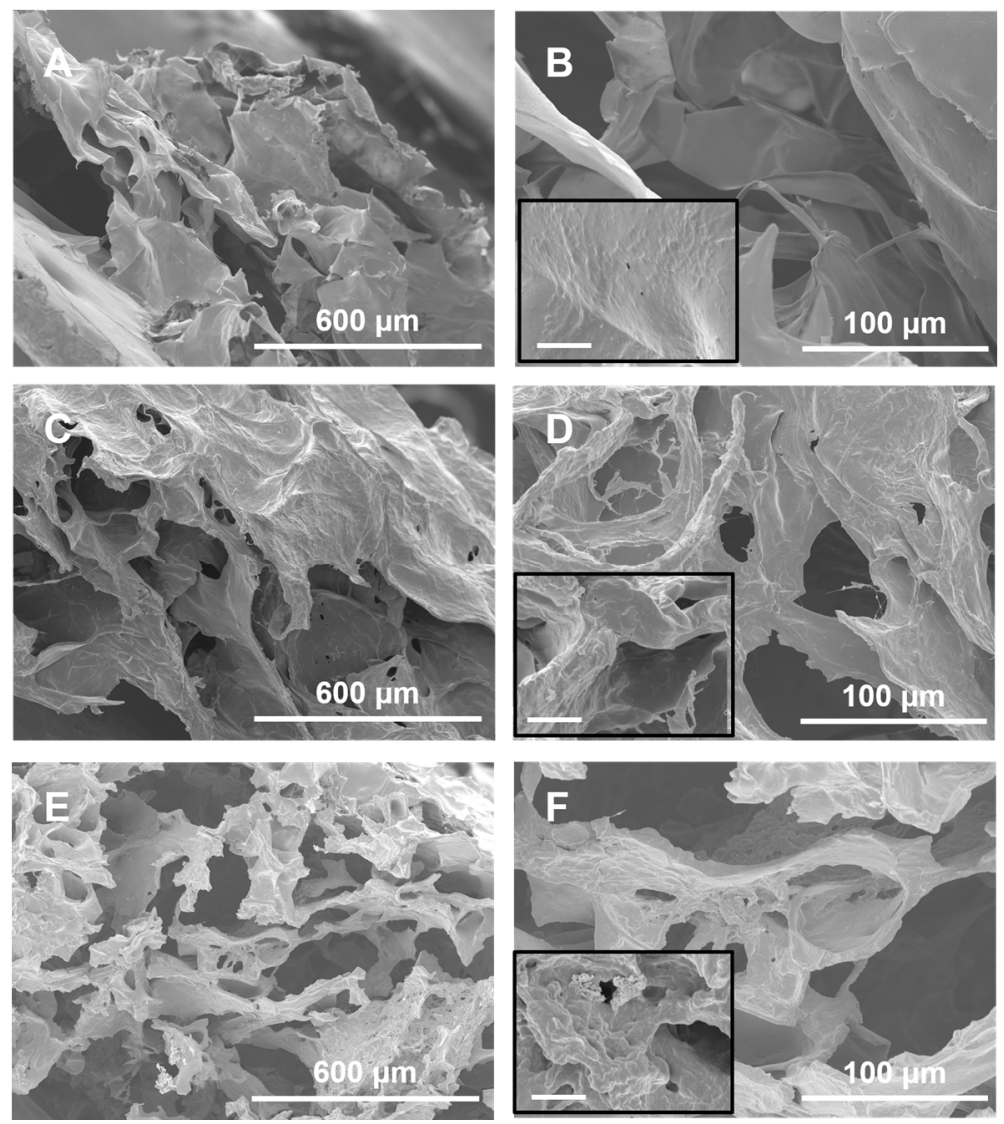

Figure 3. 52. Scanning electron microscopy of the inner structure of the poly(MeHA-co-EA) copolymer networks: MeHA:PEA 1:0 (A-B), MeHA:PEA 1:1 (C-D) and MeHA:PEA 1:10 (E-F). The images of each composition were taken for the last time point at which samples in the enzymatic solution were observed. Insets show roughness of the inner structures for each composition at high magnification (scale bar $10 \mu \mathrm{m}$ ).

Before the enzymatic degradation, the poly(MeHA-co-EA) copolymer networks showed different internal morphologies depending on the composition thereof (Figure 3. 51). The MeHA:PEA 1:0 sample displayed interconnected pores forming a sheet-like structure, which surface presented certain roughness. The MeHA:PEA 1:1 samples showed an interconnected structure forming sheets crossed by circular porous with different sizes. In this case, the roughness of the sheets resulted to be similar to that of the MeHA:PEA 1:0 sample. The MeHA:PEA 1:10 samples showed a collapsed and unstructured inner matrix distributed in agglomerates. 
The internal structure of the degraded poly(MeHA-co-EA) copolymer networks (Figure 3. 52) showed some changes respect to the non-degraded ones. The MeHA:PEA 1:0 sample showed a collapsed structured losing its interconnected structure, but its roughness seems to not be affected by the enzyme activity. The degradation in the MeHA:PEA 1:1 sample yielded a closer internal structure and the increasing of the roughness of the internal walls, however, its original internal porous structure was also observed. On the contrary, the effect of the enzyme on the MeHA:PEA 1:10 sample gave an unstructured internal matrix more open with respect to the non-degraded one. The roughness of this copolymer network resulted similar to that of the original non-degraded sample.

\subsubsection{Determination of by-products}

In order to determine the molecular weight distribution of by-products produced during the enzymatic degradation, the supernatants of the samples were collected at different time points and were analysed by gel permeation chromatography (see section 2.3). The HA solutions are usually passed through a chromatographic columns using fluxes with ionic strengths higher than $1 \mathrm{M}$ with the aim to shield electrostatic repulsions. Therefore, the supernatants were analysed using a flux of $0.15 \mathrm{M} \mathrm{NaCl}$, which also possesses similar ionic strength than the medium employed in the enzymatic solution (0.15 M PBS).

The methodology employed to determine the molecular weight of degraded $\mathrm{HA}$ chains was carried out using a calibration curve obtained from narrow standards of poly(ethylene oxide)/poly(ethyl glycol) (PEO/PEG). However, the elution volume (directly proportional to the retention time) of a molecule depends on its molecular weight, as well its hydrodynamic volume, and hence differences in the hydrodynamic volume between the polymer employed as standard and the HA should be taken into account. For this reason, the calibration curve calculated from the PEO/PEG narrow standards was reformulated using the Mark-HouwinkSakurada equations, which relate the intrinsic viscosity and molecular weight using the equation $[\eta]=\mathrm{k} \cdot \mathrm{M}^{\alpha}$ (described in section 1.2.2). Moreover, the molecular weight and the intrinsic viscosity of two components in a determined solvent may be related using the equation:

$\operatorname{Mw}_{1}\left[n_{1}\right]=M_{2}\left[n_{2}\right] \quad$ [Equation 3.7]

therefore, combining both equations the molecular weight of a component from the known one of other component may be calculated by their constants, $k$ and $\alpha$, as follows: 
$\ln M_{2}=\frac{1}{1+\alpha_{2}} \cdot \ln \frac{k_{1}}{k_{2}}+\frac{1+\alpha_{1}}{1+\alpha_{2}} \cdot \ln M_{1}$

[Equation 3.8]

the constants $k$ and $\alpha$ for PEO/PEG and HA are collected in Table 3. 12. These constants were compiled from previous works, in which they were obtained under similar conditions than the employed in the present work.

Table 3. 12. Values of the constants $k$ and $\alpha$ for $H A$ and PEO/PEG.

\begin{tabular}{cccc}
\hline Component & $\begin{array}{c}\text { Range of molecular } \\
\text { weight (kDa) }\end{array}$ & $\boldsymbol{\alpha}$ & $\mathbf{k}(\mathbf{m L} / \mathbf{g})$ \\
\hline \multirow{2}{*}{$\mathrm{HA}^{(1)}$} & below $10^{2}$ & 1.06 & $1.29 \times 10^{-3}$ \\
& from $10^{2}$ to $10^{3}$ & 0.78 & $3.39 \times 10^{-2}$ \\
& above $10^{3}$ & 0.60 & $3.95 \times 10^{-1}$ \\
PEO/PEG ${ }^{(2)}$ & from $3 \times 10^{3}$ to $7 \times 10^{5}$ & 0.82 & $0.64 \times 10^{-2}$ \\
\hline
\end{tabular}

(1) Data from [131]

(2) Data from [345]

The variation of $k$ and $\alpha$ with molecular weight should be taken into account: HA constants vary depending on the molecular weight range of the polymer chain, while the PEO/PEG ones show similar values in the range of molecular weights studied. This fact was considered in the determination of the calibration curve and the posterior analysis of supernatants.

Additionally, in order to not dismiss characteristic peaks of HA chains, the different media employed ( $0.15 \mathrm{M}$ PBS and $0.15 \mathrm{M} \mathrm{NaCl}$ containing $0.05 \%$ of sodium azide), and the enzyme in $0.15 \mathrm{M}$ PBS containing $0.05 \%$ of sodium azide at time 0 and after 56 days were also passed through the chromatographic columns. The enzyme at the employed concentration was not detected and salts from the different media appeared at times up 46 minutes. Therefore, the analysed peaks were limited to times below 46 minutes.

Taking into account all the considerations mentioned above, the supernatants of the different samples (solv_HA:DVS 1:0.9 sample, HA-DVS scaffolds and the poly(MeHA-co-EA) copolymer networks) were analysed by chromatography. For each injection, the chromatographic device proportioned raw signals with arbitrary units of the refractive index as a function of the retention time. After the normalization of the refractive index signals, the onset and offset of the detected peaks were determined and the region limited by them was integrated. From the 
integration of the corresponding peaks, the fraction $\left(w_{i}\right)$ of each specie with molecular weight $\left(\mathrm{M}_{\mathrm{i}}\right)$ was determined, and hence the molecular weight distribution was plotted. Therefore, the weight average molecular weight (Mw), the number average molecular weight (Mn) and the polydispersity index (PDI) were determined using the following equations:

$$
\begin{aligned}
& \mathrm{Mw}=\sum_{\mathrm{i}} \mathrm{M}_{\mathrm{i}} \cdot \mathrm{w}_{\mathrm{i}} \quad \text { [Equation 3.9] } \\
& M n=\frac{1}{\sum_{i} w_{i} / M_{i}} \quad \text { [Equation 3.10] } \\
& \mathrm{PDI}=\frac{\mathrm{Mw}}{\mathrm{Mn}} \quad \text { [Equation 3.11] }
\end{aligned}
$$

The following figure shows the molecular weight distribution of the by-products produced through the degrading solv_HA:DVS 1:0.9 sample at different time points:

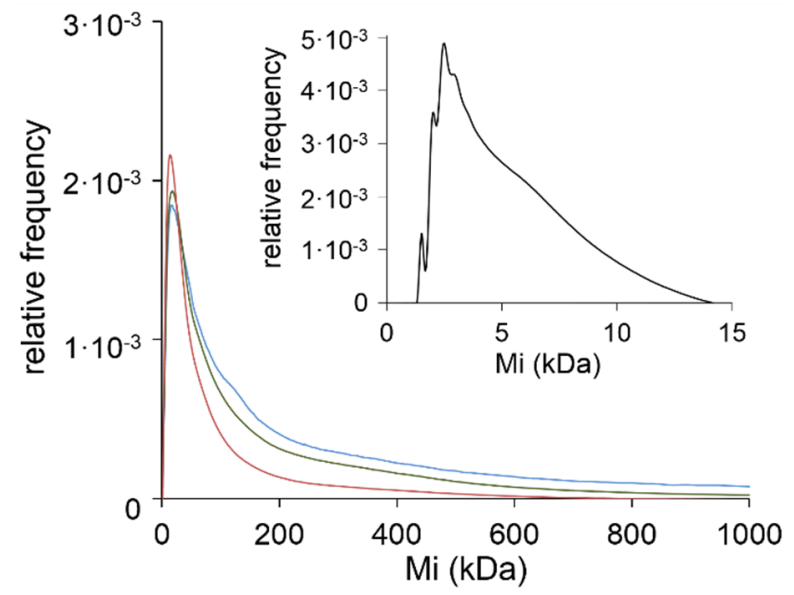

Figure 3. 53. Molecular weight distribution of degraded HA chains in the solv_HA:DVS 1:0.9 sample at different times: 6 hours (blue line), 1 day (green line) and 3 days (red line). Inset shows the molecular weight distribution after 56 days of enzymatic digestion.

The supernatants of the HA-DVS scaffolds studied by chromatography showed no traces of by-products due to enzymatic degradation in the range of times studied. However, the by-products of the degradation in solv_HA:DVS 1:0.9 samples (Figure 3.53) were observed after 6 hours of enzymatic digestion. The 
molecular weight distribution of by-products of the net-HA:DVS 1:0.9 sample after 6 hours and 3 days of enzymatic digestion resulted to be a markedly asymmetry. However, this asymmetry on the molecular weight distribution was slightly decreased for relative short incubation times (between 6 hours and 3 days). Even, this asymmetry was drastically reduced for much longer times of enzymatic degradation, up to 56 days (inset in Figure 3. 53). Moreover, all the parameters obtained from the chromatography ( $\mathrm{Mw}, \mathrm{Mn}$ and $\mathrm{PDI}$ ) were significantly decreased for longer incubation periods (Table 3. 13).

Table 3. 13. Mw, Mn and PDI of the by-products obtained in the enzymatic degradation of the solv_HA:DVS 1:0.9 sample at different incubation times.

\begin{tabular}{cccc}
\hline Incubation time & Mw $(\mathbf{k D a})$ & Mn $(\mathbf{k D a})$ & PDI \\
\hline 6 hours & $47.3 \pm 3.9$ & $14.0 \pm 0.2$ & $3.4 \pm 0.2$ \\
24 hours & $40.2 \pm 1.5$ & $13.0 \pm 0.0$ & $3.1 \pm 0.1$ \\
3 days & $28.8 \pm 0.6$ & $10.9 \pm 0.2$ & $2.7 \pm 0.1$ \\
56 days & $4.0 \pm 0.0$ & $3.1 \pm 0.0$ & $1.3 \pm 0.0$ \\
\hline
\end{tabular}

A more detailed study of the molecular weight distribution of the by-products in the degrading solv_HA:DVS 1:0.9 samples after 56 days (inset in Figure 3. 53) revealed different maximums with molecular weights of 2.9, 2.5, 2.0 and 1.6 kDa. Therefore, taking into account the molecular weight of the disaccharide unit of HA (401 Da, including the sodium ion), these maximums correspond to HA oligosaccharides between 8 - and 14-mer (4 and 7 disaccharide units, respectively).

The following figure shows the molecular weight distribution of the by-products produced through the degradation process of three different poly(MeHA-co-EA) copolymer networks at different time points: 

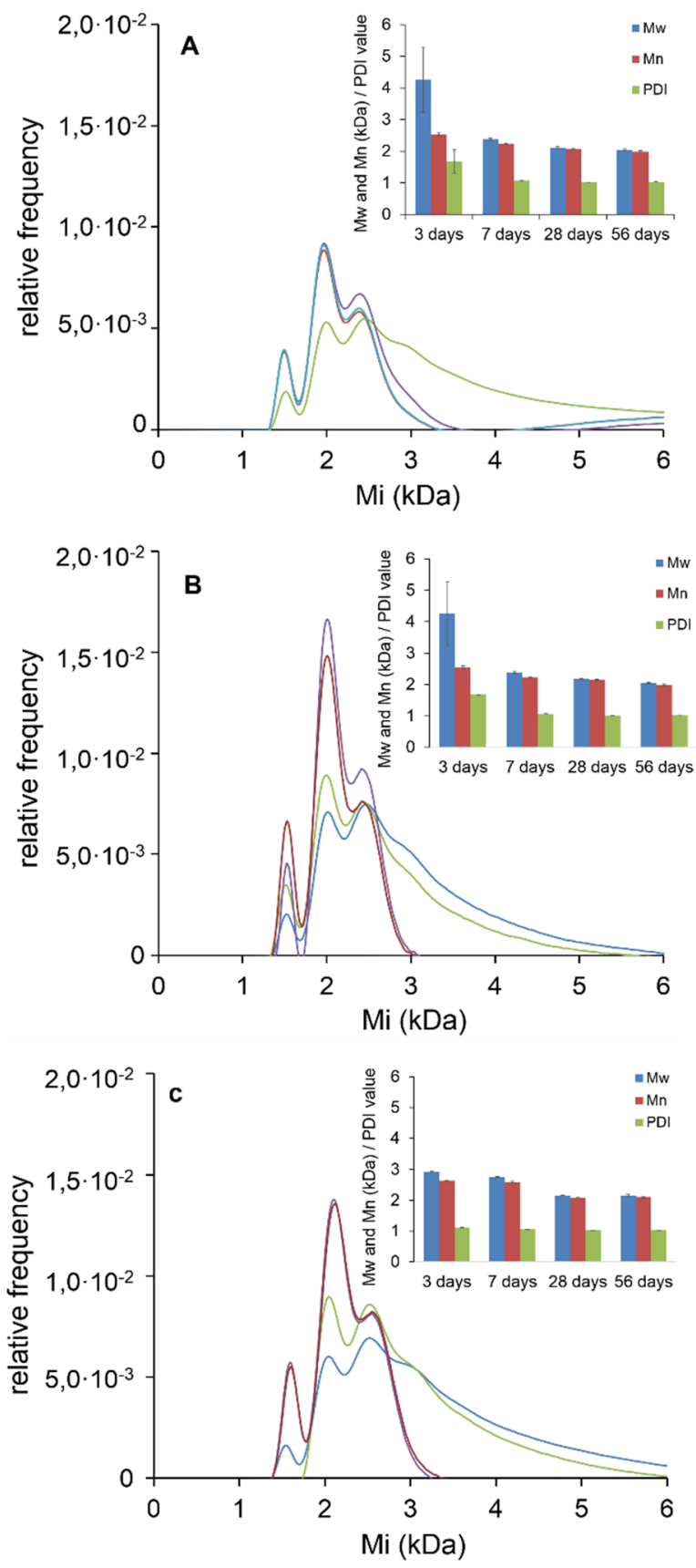

Figure 3. 54. Molecular weight distribution of degraded HA chains in MeHA:PEA 1:0 (A), MeHA:PEA 1:1 (B) and MeHA:PEA 1:10 (C) at different degradation times: 3 days (blue line), 7 days (green line), 28 days (purple line) and 56 days (red line). Insets show the parameters $M w, M n$ and $P D I$ for each composition at different degradation times. Vertical bars show the standard deviation. 
Figure 3. 54 shows the molecular weight distribution of the by-products obtained in the enzymatic degradation of MeHA:PEA 1:0, MeHA:PEA 1:1 and MeHA:PEA 1:10 samples. In general, the molecular weight distribution resulted to be asymmetric for all the samples, but this asymmetry (PDI values slightly higher than 1) was drastically reduced for incubation times up 7 days, at which time the PDI was found with values around near 1. However, in the MeHA:PEA 1:0 sample it can be observed a small fraction of by-products with $\mathrm{Mw}$ higher than $4 \mathrm{kDa}$ for all the incubation times studied. With respect to the $\mathrm{Mw}$ values, the different compositions reached values between 3 and $4 \mathrm{kDa}$ for incubation times below 7 days, however that value was significantly decreased around $2 \mathrm{kDa}$ at 28 days.

A detailed study of the molecular weight distribution shows that the different poly(MeHA-co-EA) copolymer networks displayed several peaks with molecular weights between 1 and $3 \mathrm{kDa}$. These maximums found in the molecular weight distribution displayed molecular weights around 1.6, 2.0 and $2.4 \mathrm{kDa}$, corresponding to HA oligosaccharides between 8- and 12-mer (4 and 6 disaccharide units, respectively). Moreover, another maximum in the molecular weight distribution was found with a value around $3 \mathrm{kDa}$, which corresponds with HA oligosaccharides between 14- and 16-mer (7 and 8 disaccharide units, respectively), for shorter degradation times (below 7 days). 


\subsection{Cell cultures}

Viability assays and cell cultures were performed in the HA-DVS scaffolds and poly(MeHA-co-EA) copolymer networks with the aim of determining some biological characteristics. The cell viability was addressed by different assays using extracts prepared from the materials (see section 2.4). Moreover, HA-DVS scaffolds were seeded with different cell populations as described in section 2.5.

\subsubsection{Viability assays}

The viability of hCMEC/D3, U373 and SH-SY5Y cell lines was evaluated by seeding the different cells in 96-well plates, previously coated with fibronectin. After culturing cells for 48 hours in ECBM medium, the cells were exposed to the extracts prepared from the different materials (see section 2.4). Due to the similar chemistry employed between compositions, these assays were carried out employing the extract of a representative composition for each material group: scaff_HA:DVS 1:0.9 and MeHA:PEA 1:1. The effect of the different extracts on cell viability was studied by different methods: cytotoxicity by the MTS and crystal violet assays, apoptosis by the LDH release assay, proliferation by the Ki-67 assay and inflammatory effects by the expression of E-selectin.

\section{Cytotoxicity}

The cytotoxic effect of the extracts, obtained from the biomaterials, on the different cell lines were determined by different methods: MTS, crystal violet and LDH release assay. The MTS and crystal violet are methods employed to determine live cells by indirect methods, whereas the LDH release assay is a measure of apoptotic cells. In the case of MTS and crystal violet assays, the different cell lines exposed to ECBM for 4 hours were set as the $100 \%$ viability with the aim to determine the effect of culture time in this value.

The viability of the different cell populations exposed to the different material extracts and measured by the MTS assay is displayed in the following figure: 

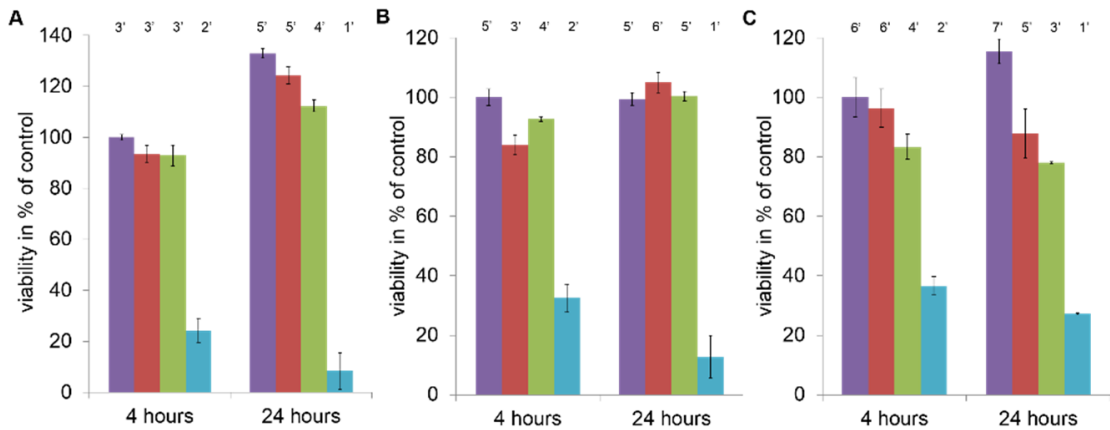

Figure 3. 55. Cell viability of U373/D3 (a), SH-SY5Y (b) and hCMEC/D3 (c) cell lines after exposure to the material extracts for 4 and 24 hours: ECBM (purple), scaff_HA:DVS 1:0.9 (red), MeHA:PEA $1: 1$ (green) and lysed cells (cyan). Viability was measured by the MTS assay, with cells cultured in pure ECBM for 4 hours being set to $100 \%$ of viability. Vertical bars show the standard deviation of each independent experiment.

The MTS assay (Figure 3. 55) showed that cells were viable at 4 and 24 hours for both material groups since the viability resulted to be up the $80 \%$ of the control. The viability of U373 and SH-SY5Y cells after 2 days of exposition to the biomaterial extract increased with respect to the control. However, the viability of hCMEC/D3 decreased after 24 hours, but the values resulted acceptable. Moreover, lysed cells displayed values of viability below $40 \%$ with respect to the control. The cells cultured with ECMB increased their viability after 24 hours with respect to culture times of 4 hours.

The viability of the different cell populations exposed to the different material extracts and measured by the crystal violet assay is shown in the following figure:
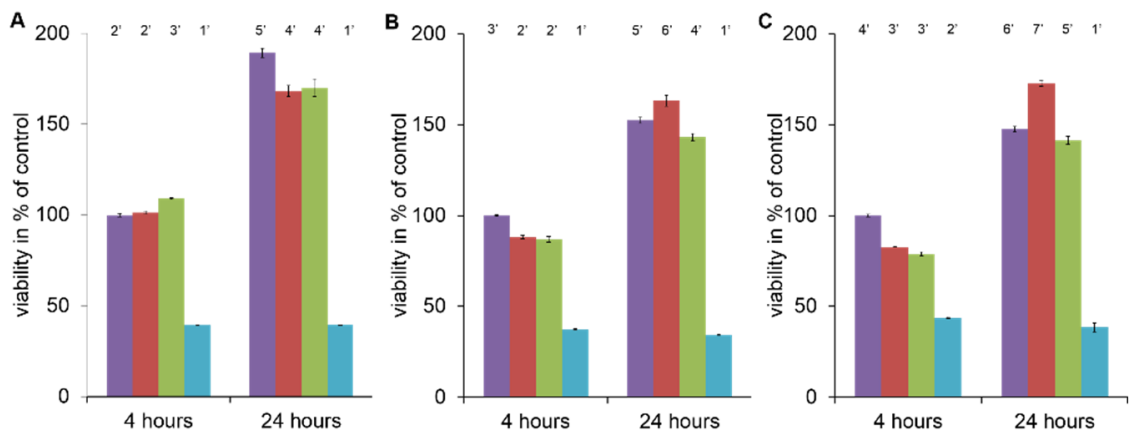

Figure 3. 56. Cell viability of U373/D3 (a), SH-SY5Y (b) and hCMEC/D3 (c) cell lines after exposure to the material extracts for 4 and 24 hours: ECBM (purple), scaff_HA:DVS 1:0.9 (red), MeHA:PEA

$1: 1$ (green) and lysed cells (cyan). Viability was measured by the crystal violet assay, with cells cultured in pure ECBM for 4 hours being set to $100 \%$ of viability. Vertical bars show the standard deviation of each independent experiment. 
Figure 3. 56 shows the cell viability measured by the crystal violet assay. The viability determined by this method resulted in similar results than those employing the MTS assay. However, in this case, all the cell lines increased their viability with respect to the corresponding controls when they were cultured in the different biomaterial extracts. As expected, the viability of lysed cells resulted in values below $40 \%$ of viability with respect to the control.

The apoptosis of the different cell populations exposed to the different material extracts and addressed from the determination of the LDH released by cells is shown in the following figure:
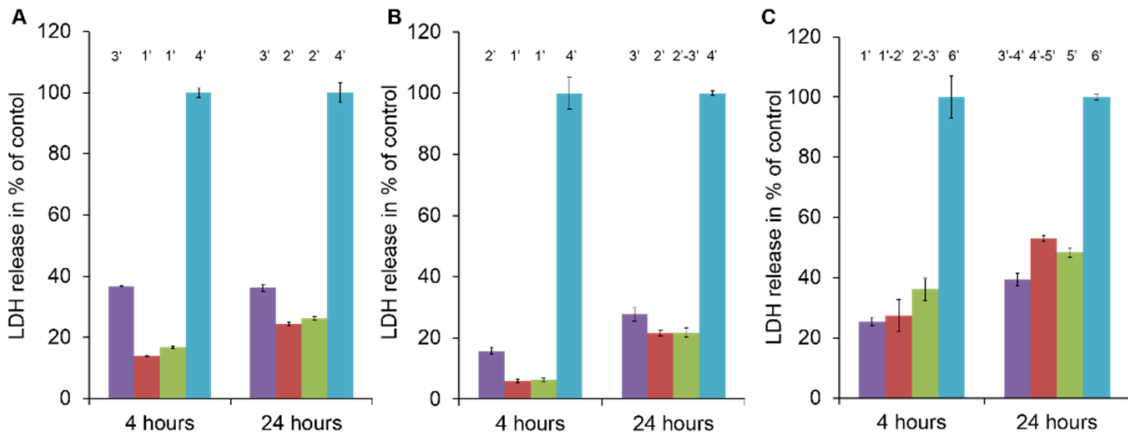

Figure 3. 57. LDH release in U373/D3 (a), SH-SY5Y (b) and hCMEC/D3 (c) cell lines after exposure to the material extracts for 4 and 24 hours: ECBM (purple), scaff_HA:DVS 1:0.9 (red), MeHA:PEA 1:1 (green) and lysed cells (cyan). Cell apoptosis was measured by the LDH release assay, with lysed cells after culture for 24 hours being set to $100 \%$ of apoptosis. Vertical bars show the standard deviation of each independent experiment.

As can be observed in Figure 3. 57, the release of LDH by cells exposed to the different media resulted in values below $50 \%$ (taking as controls cells exposed to ECMB for 4 and 24 hours). After 4 hours of culture, the different cell lines showed a percentage of LDH release below $40 \%$, whereas this percentage slightly increase after 24 hours. In the case of U373 and SH-SY5Y cell lines, the $\mathrm{LDH}$ release of cells exposed to the material extracts was lower with respect to cells cultured in ECBM. Oppositely, the hCMEC/D3 cell line displayed a LDH release when exposed to the different extracts and ECBM higher than the other cell lines. This effect was increased for hCMEC/D3 cultured for 24 hours. 


\section{Proliferation}

In order to determine the effect of the material extracts on cell proliferation, the amount of nuclear Ki-67 was quantified. In this case, cultures in ECBM after 4 hours were set as the $100 \%$ of the proliferation with the aim of determining changes induced by the culture time. The effect of material extracts on the proliferation of the different cell populations is shown in the following figure:
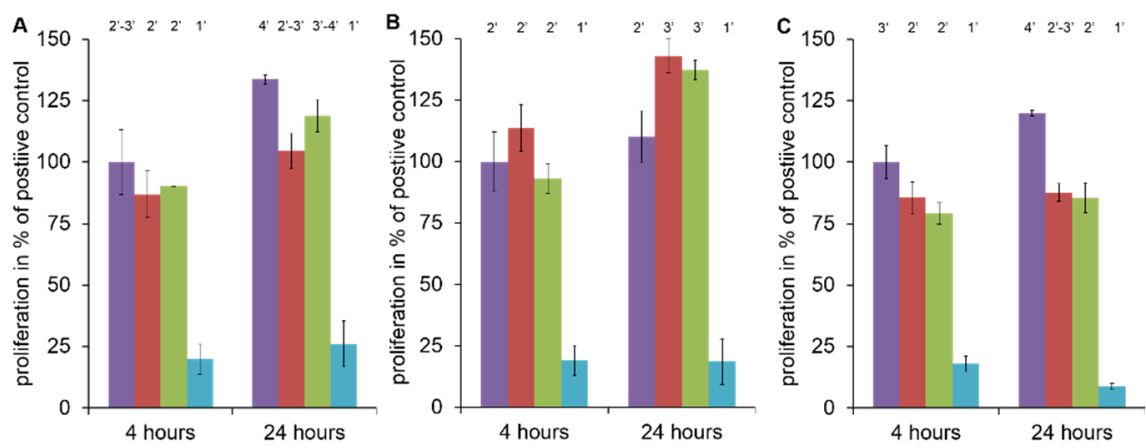

Figure 3. 58. Cell proliferation of U373 (a), SH-SY5Y (b) and hCMEC/D3 (c) after exposure to the material extracts for 4 and 24 hours: ECBM (purple), scaff_HA:DVS 1:0.9 (red), MeHA:PEA 1:1 (green) and lysed cells (cyan). Proliferation was measured by the ki-67 assay, with cells cultured in pure ECBM for 4 hours being set to $100 \%$ of proliferation. Vertical bars show the standard deviation of each independent experiment.

Figure 3. 58 shows that cell proliferation was increased after 24 hours in culture for all cell lines and exposure media, including ECBM. This increment on proliferation resulted to be significant for U373 cell line exposed to all the mentioned media, SH-SY5Y cell line exposed to the different extracts of materials and hCMEC/D3 cell line in ECBM. In the case of lysed cells, the proliferation value was below $30 \%$ with respect to the control.

\section{Inflammation}

In order to determine the inflammatory effect of extracts obtained from the biomaterials on the hCMEC/D3 cell line, the E-selectin expression was evaluated by a CAM-EIA assay. The hCMEC/D3 cell line was exposed for 4 hours to both material extracts, ECBM was used as negative control and ECBM containing 1 $\mu \mathrm{g} / \mathrm{mL}$ of LPS was employed as positive control (set as $100 \%$ of inflammation). 


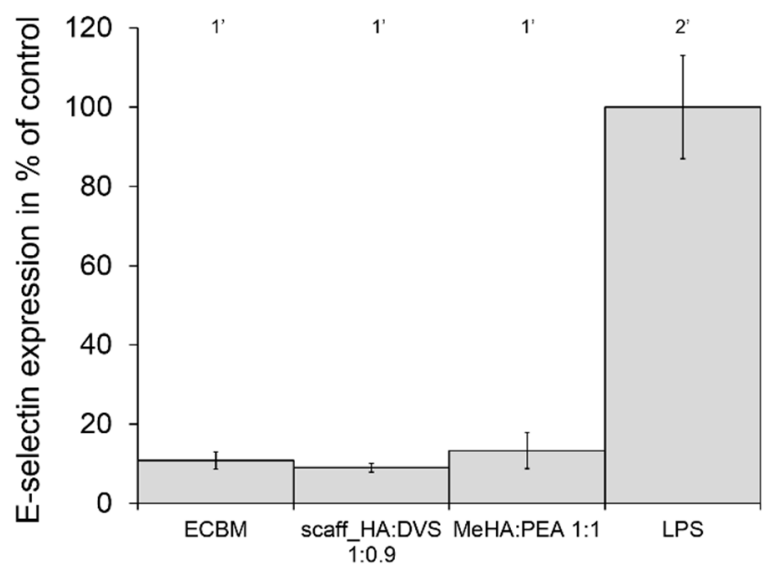

Figure 3. 59. Inflammation processes on $\mathrm{hCMEC/D3}$ cell line exposed to the material extracts for 4 hours. Inflammation was measured determining the expression of E-selectin using a CAM-EIA assay, with cells cultured in ECBM containing $1 \mu \mathrm{g} / \mathrm{mL}$ of LPS for 4 hours being set to $100 \%$ of inflammation. Vertical bars show the standard deviation.

Figure 3. 59 shows the inflammatory effects of the different extracts and media employed as controls on the hCMEC/D3 cell line. The expression of E-selectin in the hCMEC/D3 cell line resulted in values below $15 \%$ with respect to cells stimulated with $1 \mu \mathrm{g} / \mathrm{mL}$ of LPS.

\subsubsection{Cultures of cell populations on scaffolds}

Influence of the degree of crosslinking on the initial cell colonization

In order to determine the influence of the HA:DVS molar ratio on the initial cell colonization, the U373, SH-SY5Y and hCMEC/D3 cell lines were seeded on the different HA-DVS scaffolds. The different cultures were carried out up 7 days and the bottom of 96-well plates were taken as controls with the aim to assess the health of the cultured cells.

The culture of each cell type (U373 (Figure 3. 60), SH-SY5Y (Figure 3. 61) and hCMEC/D3 (Figure 3. 62)) on the different HA-DVS scaffolds and controls is shown in the following figures: 

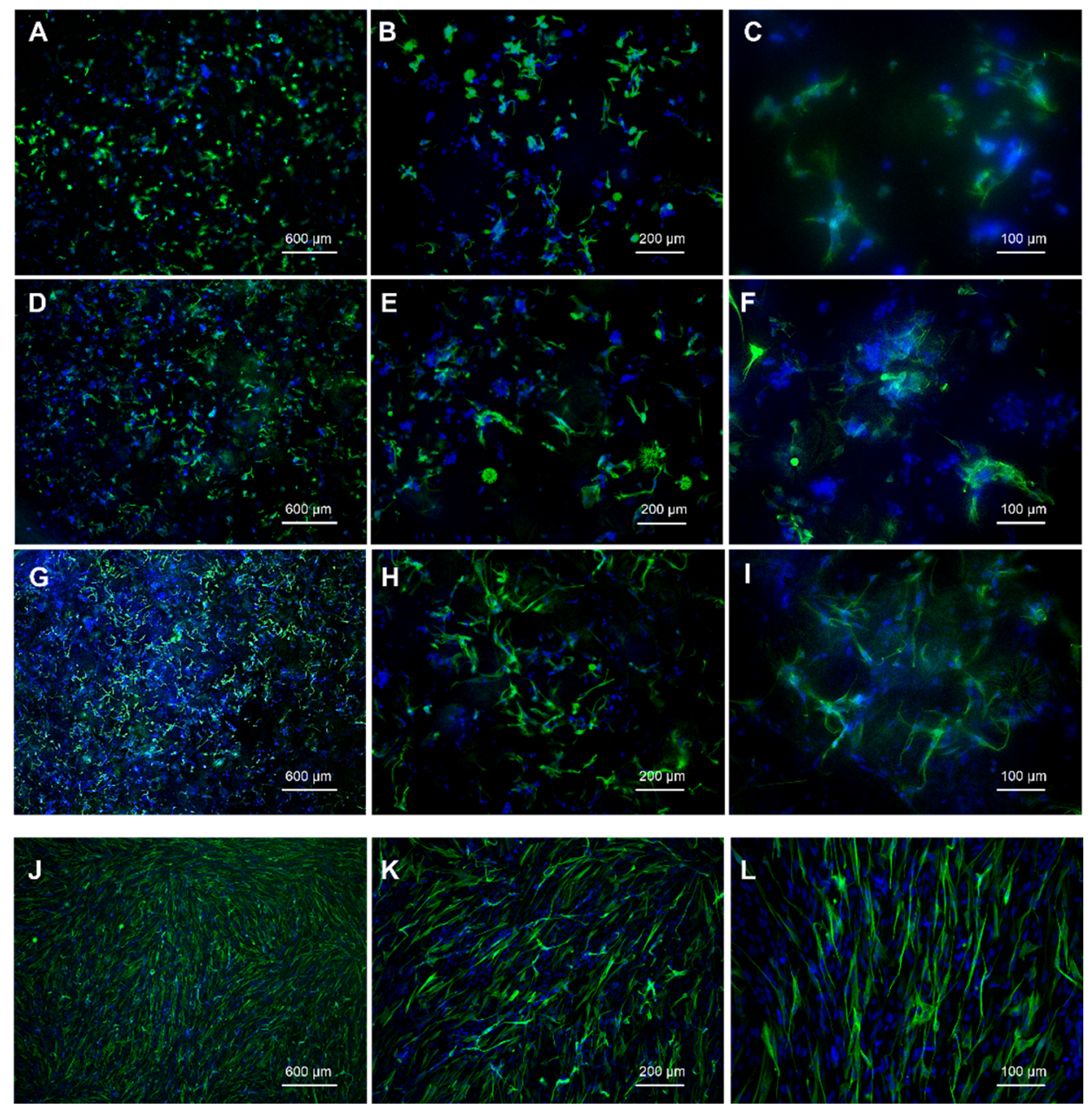

Figure 3. 60. GFAP expression (green fluorescence) by U373 cell line cultured on scaff_HA:DVS 1:0.6 (A, B and C), scaff_HA:DVS 1:0.9 (D, E and F) and scaff_HA:DVS 1:1.5 (G, H and I), and 96well plates $(\mathrm{J}, \overline{\mathrm{K}}$ and $\mathrm{L})$ for 7 days. Cell nuclei were stained in blue with Hoechst. 

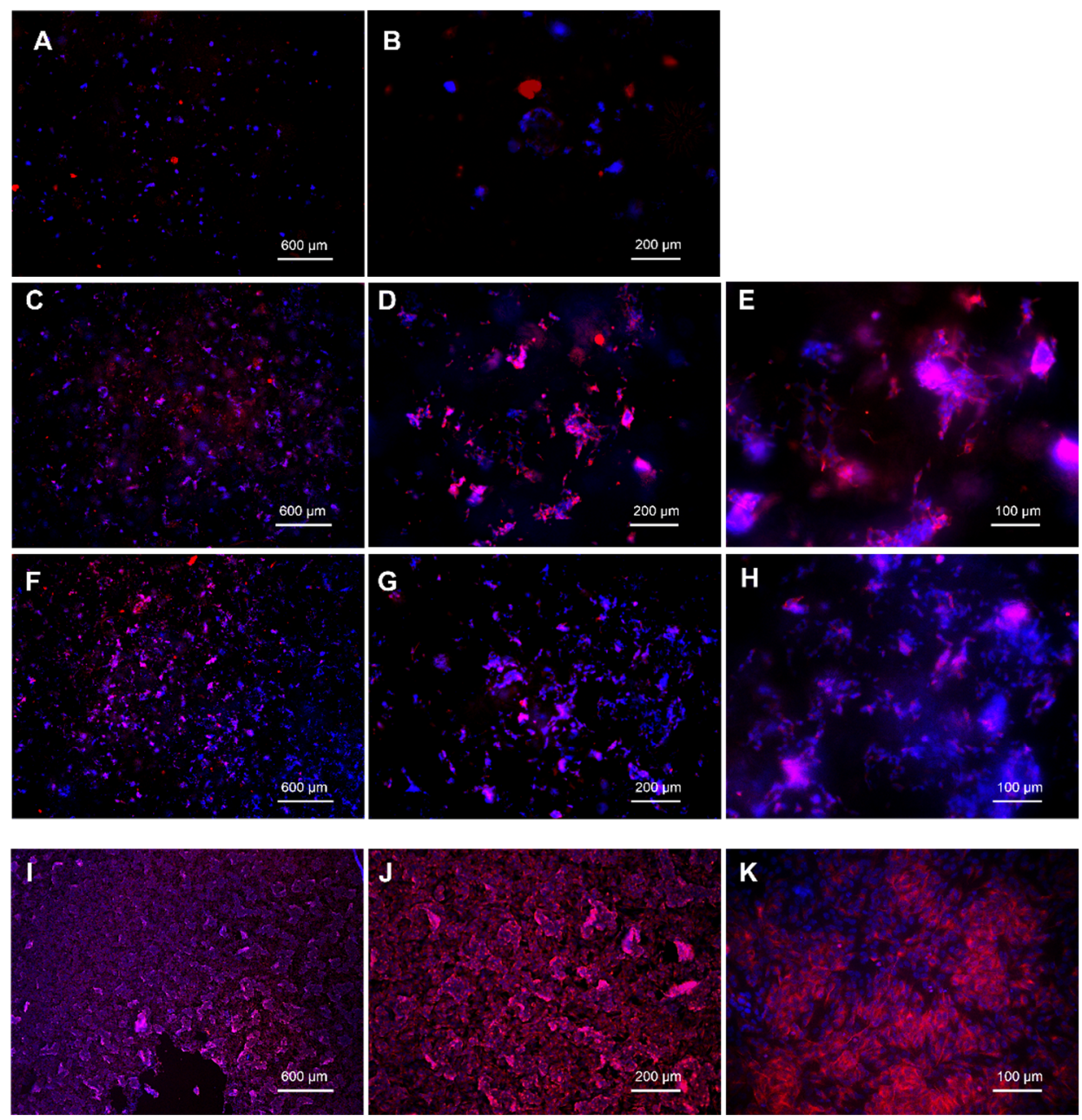

Figure 3. 61. Tuj-1 expression (red fluorescence) by SH-SY5Y cell line cultured on scaff_HA:DVS 1:0.6 (A, and B), scaff_HA:DVS 1:0.9 (C, D and E) and scaff_HA:DVS 1:1.5 (F, G and H), and 96well plates (I, $\mathrm{J}$ and $\mathrm{K}$ ) for 7 days. Cell nuclei were stained in blue with Hoechst. 

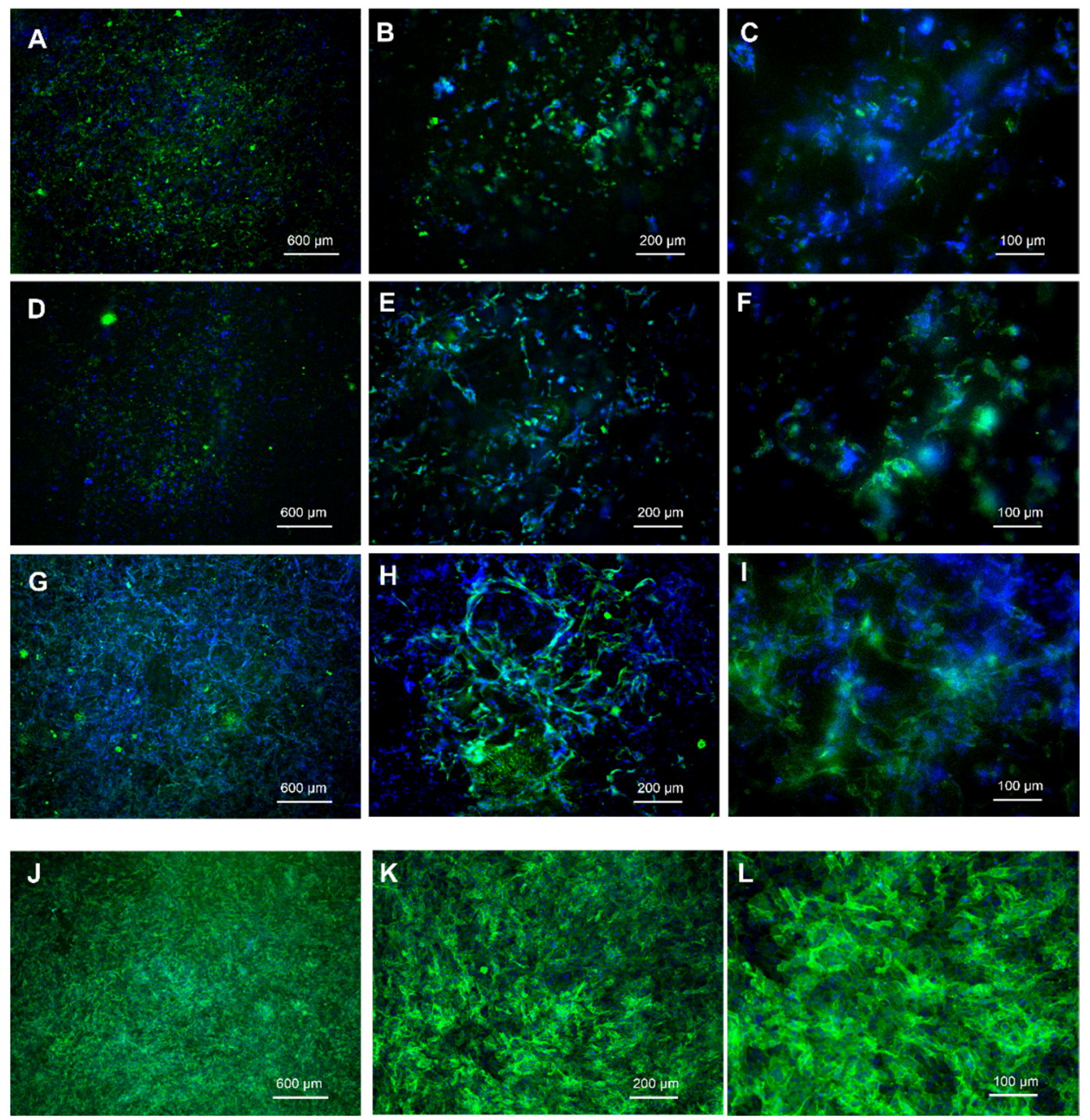

Figure 3. 62. $C D-31$ expression (green fluorescence) by hCMEC/D3 cell line cultured on scaff_HA:DVS 1:0.6 (A, B and C), scaff_HA:DVS 1:0.9 (D, E and F) and scaff_HA:DVS 1:1.5 (G, H and $\mathrm{I})$, and 96 -well plates $(\mathrm{J}, \mathrm{K}$ and $\mathrm{L})$ for 7 days. Cell nuclei were stained in blue with Hoechst.

In general, the HA-DVS scaffolds showed a low cell colonization of the different cell types (U373, SH-SY5Y and hCMEC/D3 cell lines) and cells were observed on top of the scaffolds with some cell ingrowth in the adjacent layers. The different monocultures show that the initial cell colonization is clearly influenced by the amount of DVS; all cell types were more abundant in the scaff_HA:DVS 1:1.5 samples. The U373 and SH-SY5Y cells were distributed as isolated clusters, whereas the hCMEC/D3 cells in the scaff_HA:DVS 1:1.5 samples were arranged in a uniform and continuous layer surrounding the pores. 
The U373 cells cultured on the 96-well plates displayed an orientated and elongated shape. Despite the low colonization in the HA-DVS scaffolds, this elongated shape was observed in the different scaffolds, showing longer processes when they are cultured in scaffolds with higher amounts of DVS. On the contrary, the SH-SY5Y cells formed clusters in both 96-well plates and the different scaffolds, showing some short processes. The hCMEC/D3 cells cultured on $\mathrm{p}-96$ well plates were distributed as a disordered monolayer, while its culture on the scaff_HA:DVS 1:1.5 samples evoked a distribution on the porous structure, showing cell-cell connections through cell processes.
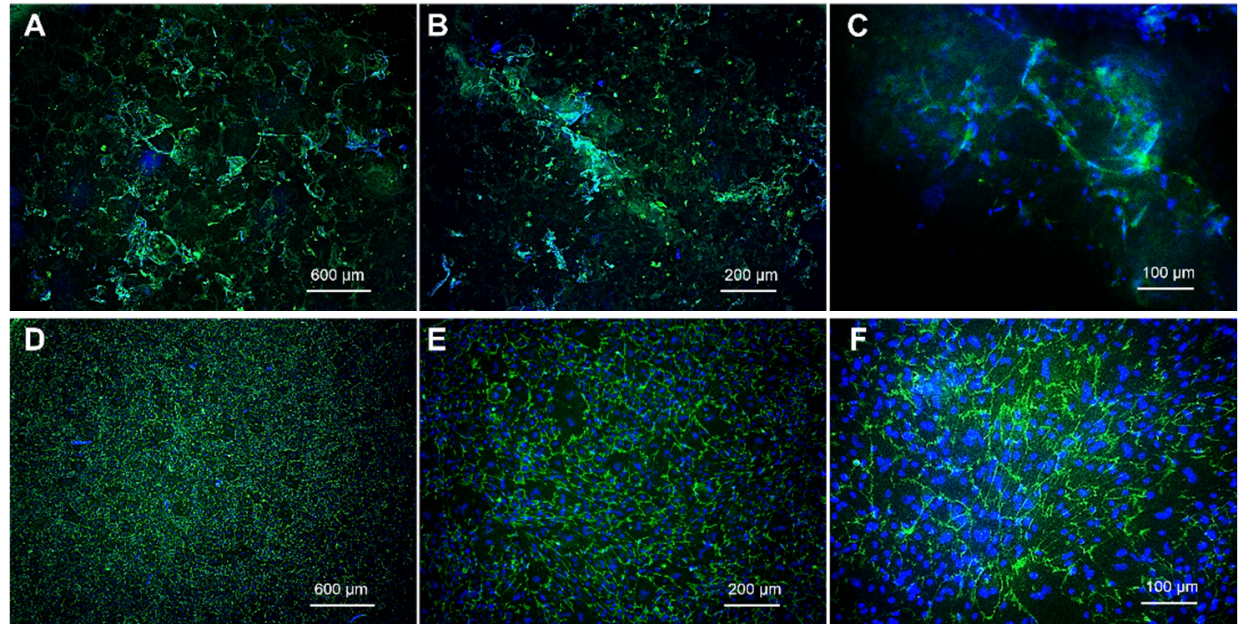

Figure 3. 63. CD-31 expression (green fluorescence) by pBECs primary cell line cultured on scaff_HA:DVS 1:1.5 (a, b and c) and 96-well plates (d, e and f) for 7 days. Cell nuclei were stained in blue with Hoechst.

Aimed by the results by the previous cultures, the primary endothelial cells, pBECs, were cultured on the scaff_HA:DVS 1:1.5 samples, taking cultures on 96well plates as control to address the health of cells. The culture of pBECs on the 96-well plates gave rise to the formation of a monolayer where cells formed a closely structure. However, as the case of hCMEC/D3 cells, the pBECs cultured on the scaff_HA:DVS 1:1.5 samples resulted to have a uniform colonization on top of the materials showing some degree of ingrowth into the scaffolds.

\section{Co-cultures and triple-cultures}

The endothelial cells in brain tissue are arranged forming a unique structure, the BBB, whose phenotypic maintenance is influenced by the interaction of endothelial cells with astrocytes and neurons among other cell types. Therefore, 
these three types of cells were employed in co-cultures and triple-cultures with the aim to study their synergistic effects on the initial colonization of the HA-DVS scaffolds. After choosing the scaff_HA:DVS 1:1.5 samples as those with the best chemistry in terms of monocultures behaviour, co-cultures and triple-cultures were performed on these scaffolds (described in section 2.5). The different cocultures and triple-cultures on the scaffolds were carried out with the aim to study the behaviour of cells, which are typical of brain tissue (endothelial, glial and neural cells).
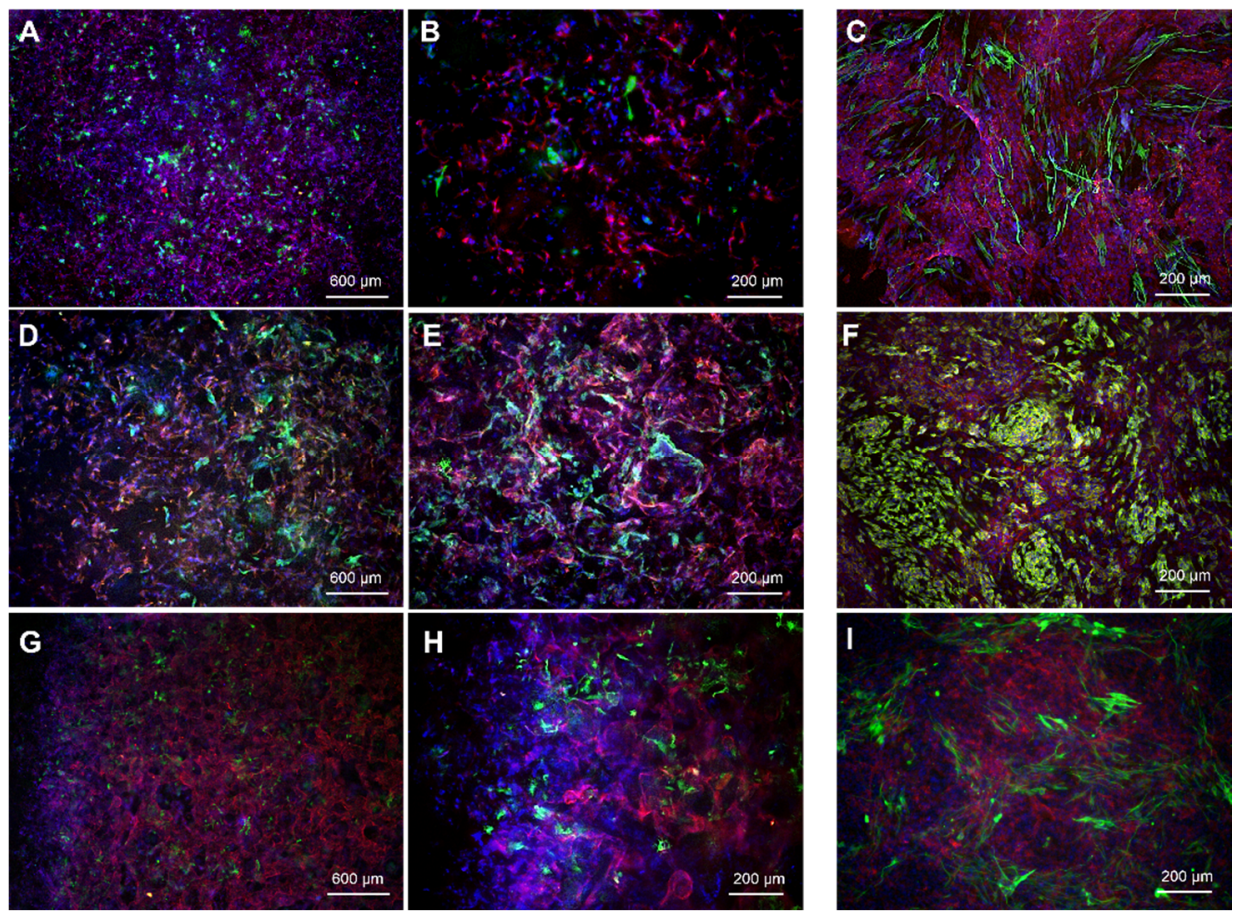

Figure 3. 64. Immunofluorescence analysis on co-cultures of hCMEC/D3 and U373 (A, B and C), hCMEC/D3 and SH-SY5Y (D, E and F) and triple-cultures of hCMEC/D3, U373 and SH-SY5Y (G, H and I). Cells were cultured on scaff_HA:DVS 1:1.5 samples (left and central columns) and 96-well plates (right column) for 7 days. hCMEC/D3 were stained against CD-31 expression (red fluorescence), U373 were stained against GFAP expression (green fluorescence) and SH-SY5Y were stained against Tuj-1 expression (green fluorescence). Cell nuclei were stained in blue with Hoechst.

In general, the initial cell colonization of the scaffolds by the hCMEC/D3 cells in co-cultures and triple-cultures after 7 days resulted more significant than the colonization observed by astrocytes and neurons (Figure 3. 64). The hCMEC/D3 cells resulted to cover few layers of the scaffolds showing cells attached onto 
their inner porous surfaces. On the contrary, the U373 cell line showed that these cells remained isolated in both co-cultures and triple-cultures. The presence of SH-SY5Y cells in the co-cultures is slightly higher compared with co-cultured U373, but these cells are forming individual clusters. However, the hCMEC/D3 co-cultured with the SH-SY5Y cells and in triple-cultures resulted in a uniform layer showing a dense distribution of cells.

The co-cultures and triple-cultures in the 96-well plates evidenced similar structures between endothelial cells with U373 and SH-SY5Y cells. The hCMEC/D3 cells formed dense layers of cells and delimited by the U373 and SHSY5Y cells yielding to heterogeneous cultures and some degree of structuration between cell groups.
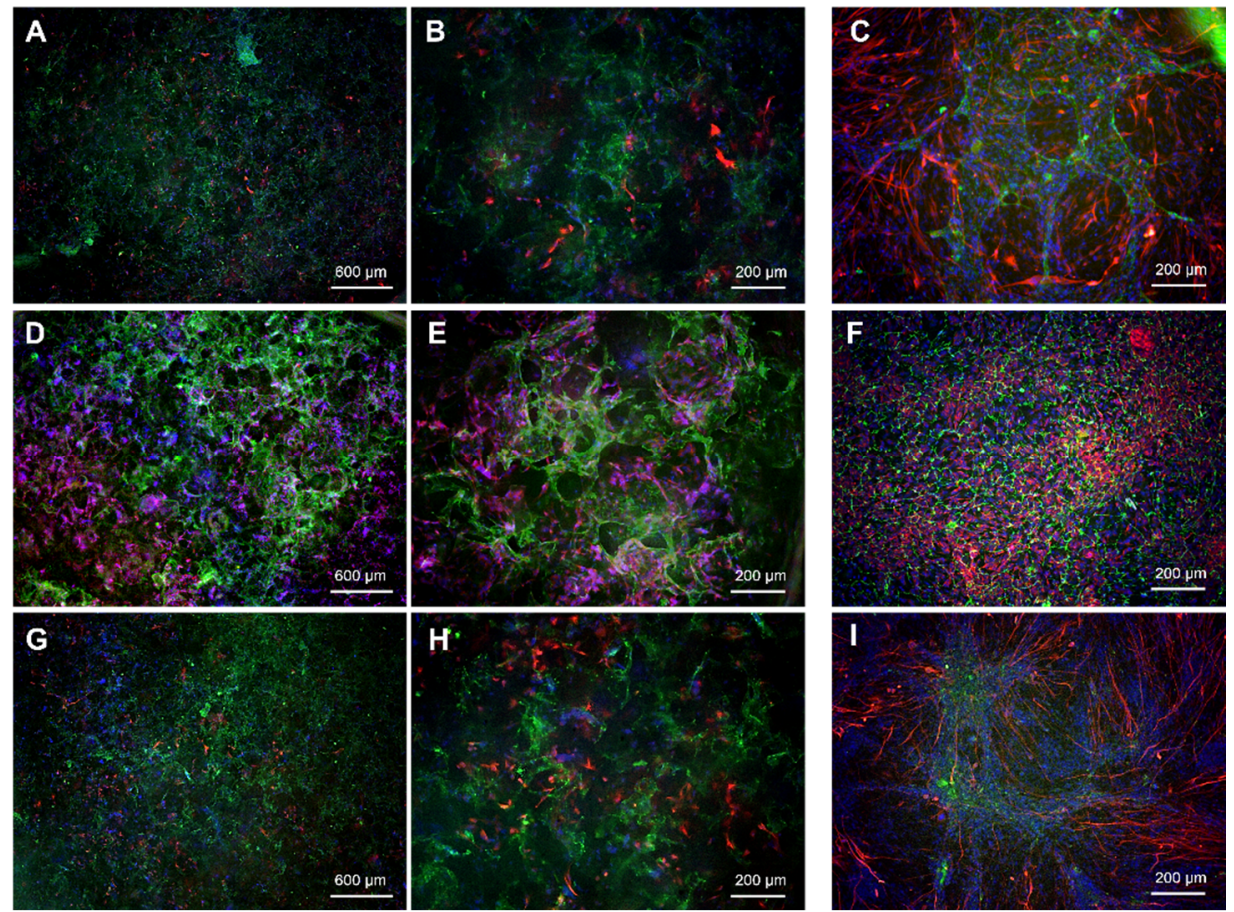

Figure 3. 65. Immunofluorescence analysis on co-cultures of pBECs and $U 373(A, B$ and $C)$, pBECs and SH-SY5Y (D, E and F) and triple-cultures of pBECs, U373 and SH-SY5Y (G, H and I). Cells were cultured on scaff_HA:DVS 1:1.5 samples (left and central columns) and 96-well plates (right column) for 7 days. The pBECs primary cells were stained against CD-31 expression (green fluorescence), U373 were stained against GFAP expression (red fluorescence) and SH-SY5Y were stained against Tuj-1 expression (red fluorescence). Cell nuclei were stained in blue with Hoechst.

The primary cells, pBECs, in co-cultures and triple-cultures resulted to give more dense populations surrounding the pores of the scaffolds (Figure 3. 65). As in 
cultures with hCMEC/D3 cells, the co-cultures and triple-cultures of U373 with pBECs on the scaffolds resulted in a low expression of these astrocyte-like cells. On other hand, the structures formed by $\mathrm{U} 373$ and pBECs resulted to be similar than those observed using the hCMEC/D3 cells; both cells types form heterogeneous structures limited by themselves. However, the SH-SY5Y cells co-cultured with pBECs yielded to a homogeneous distribution of both cell types, even observing the formation of a uniform layer of pBECs in the case of cocultures in 96-well plates.

After 14 days of culture, the different co-cultures and triple-cultures using the $\mathrm{hCMEC/D} 3$ cell line and the pBECs primary cells displayed similar results than at 7 days (Figure 3. 66). However, the different endothelial cells increased their density within the scaffolds, but this effect was not observed for U373 and SHSY5Y populations.
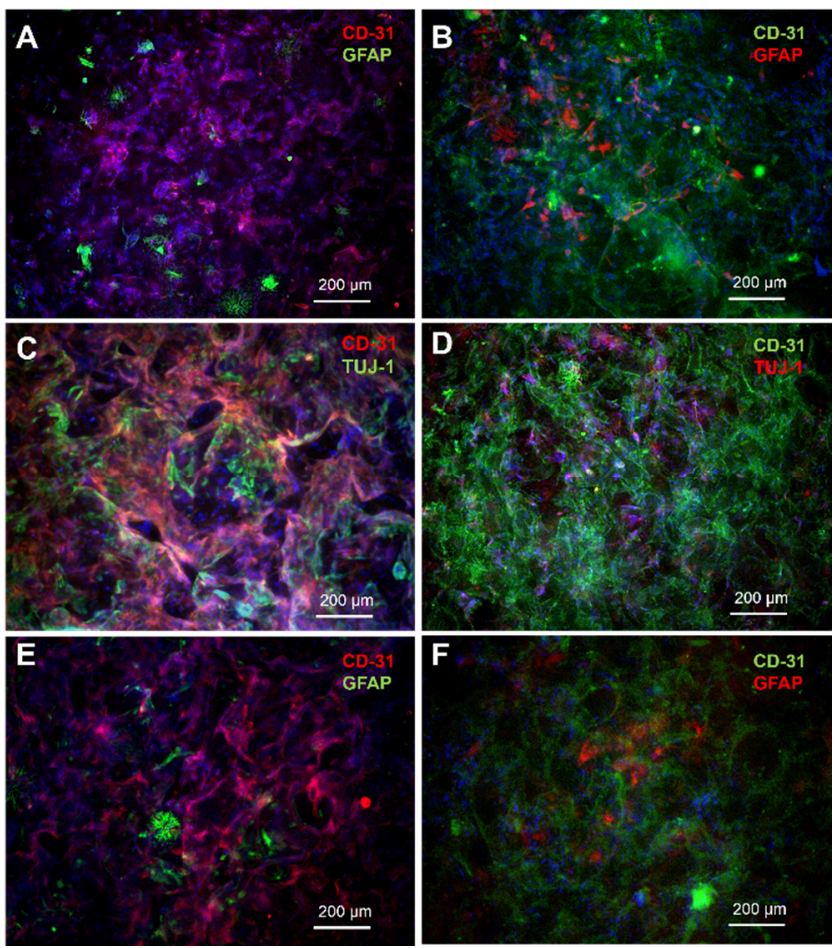

Figure 3. 66. Immunofluorescence analysis on co-cultures of hCMEC/D3 and U373 (a) pBECs and U373 (b), hCMEC/D3 and SH-SY5Y (c), pBECs and SH-SY5Y (d), and triple-cultures of hCMEC/D3, U373 and SH-SY5Y (e) and pBECs, U373 and SH-SY5Y (f). Cells were cultured on scaff_HA:DVS 1:1.5 samples for 14 days. The pBECs primary cells and hCMEC/D3 were stained against CD-31 expression, U373 were stained against GFAP expression and SH-SY5Y were stained against Tuj- 1 expression. Cell nuclei were stained in blue with Hoechst. The SH-SY5Y cell line was unstained in triple-cultures. 
CHAPTER IV

DISCUSSION 

The properties of HA and its nature as component of the ECM within multiple tissues have encouraged researchers to employ it for many tissue engineering purposes. In the past decades, the research of HA has found some clinical applications: temporal substitute of the synovial fluid in osteoarthritic joints, protective fluid against minor surgeries (ophthalmologic surgery), anti-adherence in some surgeries and as a dermal filler in aesthetic surgery [261,365]. However, the direct application of HA solutions is limited in tissue engineering since HA shows a high bioresorption within the body because of its high solubility, a low mechanical properties in its use as transient artificial ECM in regenerating tissues and an high biodegradability due to the presence of enzymes and chemicals within the body. Moreover, HA with high molecular weight is usually preferred to the one of low molecular weight because of its lower inflammatory effects and longer residence times. However, the HA with low molecular weight possesses some beneficial effects such as angiogenic activity helping the transport of nutrients in the regenerating tissue. Taking into account this duality, implants based on HA with high molecular weight with controlled biodegradability and insolubility in the physiological medium are usually preferred.

From the past few decades to present, many researchers have focused on developing new HA based materials with the aim of expanding the applications for tissue engineering. In this sense, a wide range of $\mathrm{HA}$ derivatives have been developed obtaining insoluble materials in physiological conditions with tailored degradability, mechanical properties and swelling degree. Therefore, a broad range of $\mathrm{HA}$ derivatives have been developed employing different chemistries and physical methods. The most common modified HA products are the ones obtained by crosslinking reactions (DVS-, glutaraldehyde- and BDDEcrosslinked); even these HA derivatives are the most common employed in several clinical applications [197].

In the present work, HA has been modified with the aim of developing polymer networks for soft tissue engineering, particularly for central nervous system regeneration in brain damages such as traumatic brain injury and stroke. Here two different strategies have been employed: the crosslinking of HA with DVS (HA-DVS polymer networks) and the copolymerization of a HA derivative, MeHA, with EA (poly(MeHA-co-EA) copolymer networks). These HA-based materials have been developed to increase the molecular weight of HA single chains, and improve and grant a control over mechanical properties, residence time in the physiological environment, degradation rate, and swelling degree. Additionally, these HA derivatives have been employed to develop structured materials for their use as artificial ECM for cells in the regenerating tissue. Therefore, the materials were subjected to a preliminary biologically characterization in vitro employing different cells belonging to the brain tissue (astrocytes, neurons and endothelial cells). 
On the other hand, the methacrylated $\mathrm{HA}, \mathrm{MeHA}$, allows its copolymerization with other monomers. In this case, MeHA has been copolymerized with EA to obtain semi-biodegradable copolymers combining the properties of HA and PEA. Therefore, the copolymers are expected to possess the biological properties of HA and the good mechanical properties and cell compatibility of PEA. Besides, different compositions of these copolymers may result in materials with tuneable mechanical properties and swelling degrees, provide structural stability during the regenerative process and improve the initial colonization of glial, neural and endothelial cells belonging to the brain tissue. 


\subsection{Crosslinking of hyaluronan with divinyl sulfone}

The divinyl sulfone-crosslinked hyaluronan, HA-DVS, (known as hylan) has been widely employed with the aim to develop HA-based hydrogels for tissue engineering applications. Moreover, this material is currently employed in some clinical applications [219]. In the present work HA-DVS polymer networks have been developed focusing the attention on their further use as artificial ECM for brain tissue engineering. In the current section is discussed the characterization of the HA-DVS polymer networks, in which water removal was performed by a solvent-casting process.

The HA-DVS crosslinking was evaluated as a method to obtain insoluble hydrogels based on HA as a principal component. The crosslinking reaction of HA with DVS requires alkaline aqueous media (above 12) since vinyl groups of DVS are firstly activated by hydroxyl ions and hydroxyls of HA must be deprotonated. It is widely accepted that this reaction occurs principally by the unique primary hydroxyl within the $\mathrm{N}$-acetyl-D-glucosamine moiety due to the steric hindrance of the molecule. Therefore, the crosslinking yields an ether bond between two HA disaccharides by one molecule of DVS (see Figure 1.6). The steric hindrance of $\mathrm{HA}$ molecule together the high viscosity and low concentration of the HA solutions yield to very low efficiencies of reaction. For this reason, the crosslinking with DVS is commonly carried out employing a DVS excess.

In order to determine the effect of DVS in the final properties of the hydrogel, different amounts of DVS were employed to carry out the crosslinking of HA. Therefore, the appropriate amounts of DVS in the development of the HA-DVS polymer networks were addressed by using a broad range of molar ratios of $\mathrm{HA}$ monomeric unit to DVS (HA:DVS molar ratio) from 1:0.2 to 1:2. Then, the resulting hydrogels were swollen in $0.15 \mathrm{M}$ PBS until reaching the equilibrium swelling (after 3 days in immersion) and the equilibrium water content, $E W C$, was evaluated with the aim to choose three representative compositions of the HADVS polymer networks for further studies. The fitting of the EWC values for the different HA-DVS polymer networks (Figure 3.1) shows a clear reduction of water in the inner network due to larger amounts of crosslinking density. However, extreme HA:DVS molar ratios gave anomalous results. The HA-DVS polymer networks with a molar ratio 1:0.2 yielded unexpected low EWC values, because of the inefficiency of the reaction and a partial solubility of the uncrosslinked HA chains. On the other hand, high HA:DVS molar ratios (above 1:1.5) resulted in heterogeneous hydrogels due to the self-polymerization of divinyl sulfone molecules. For this reason, the HA:DVS molar ratios selected were the intermediate ones: 1:0.6, 1:0.9 and 1:1.5. 
The HA molecule is subjected to spontaneous hydrolysis in aqueous media by the nucleophilic attack of water molecules on carbon forming the glycosidic bonds. However, the hydrolysis rate is increased under extreme $\mathrm{pH}$ values, both acid and alkaline [138]. The hydrolysis produces a reduction of the molecular weight of HA chains that may produce inflammatory effects and some alterations in the crosslinking reaction. Therefore, the effect of the alkaline medium, employed in the crosslinking reaction, on the molecular weight of HA was studied by chromatography (Figure 3. 2 and Figure 3. 23). The results evidence a reduction in the molecular weight during the solubilisation of HA in the alkaline medium, halving its value with respect to the original one. However, the molecular weight distribution shows a more symmetric fashion after mixing with alkaline medium, suggesting that the reduction of the molecular weight occurs in a random fashion. Moreover, the reduction in the molecular weight resulted to be to be independent of the concentration of HA in the alkaline solution. Despite the reduction of the molecular weight, the values between $700-800 \mathrm{kDa}$, which may be considered as HA chains of high molecular weight [189]. On the other hand, alkaline medium may yield other undesirable reactions within the HA chains, including the deacetylation of the acetylamide moiety [138]. However, spectra recorded from thin films of the different HA-DVS polymer networks (Figure 3. 3) showed no evidence of primary amine formation, since the double peak with maximums around 3300 and $3100 \mathrm{~cm}^{-1}$ associated to the $\mathrm{N}-\mathrm{H}$ stretching of this group was not present, and hence these secondary reactions were discarded.

The crosslinking reaction with DVS is considered complete after a few hours; however, in the present work, the reactions were carried out for 24 hours. After the crosslinking reaction, hydrogels are formed because samples show a soft but consistent appearance. Moreover, the subsequent purification processes do not produce visible disaggregation of the samples. The spectra of the HA-DVS polymer networks showed the incorporation of sulfone groups in the hydrogels (Figure 3. 3). The sulfone characteristic asymmetric and symmetric stretching appears in the self-polymerized DVS (pDVS) at wavenumbers of $1290 \mathrm{~cm}^{-1}$ and $1110 \mathrm{~cm}^{-1}$, respectively. In the case of the HA-DVS polymer networks these bands clearly appeared, but they are displaced to lower wavenumbers and, in the case of the $\mathrm{S}=\mathrm{O}$ symmetric stretching, the band was overlapped with characteristic peaks of HA. Moreover, the elemental analysis (atomic weight fraction of carbon, nitrogen, hydrogen and sulphur collected in Table 3. 6) corroborated the presence of sulphurs in the samples, even this method shows an increment of the sulphur content when the DVS is increased during the crosslinking reaction.

The swelling of crosslinked hydrogels, both neutral and polyelectrolytes, are governed by the interaction of the solvent-polymer system and the elastic properties of the crosslinked networks, which are correlated with the incorporated 
crosslinks. However, in the case of polyelectrolyte hydrogels, the presence of ionisable groups noticeably influences the swelling process. Therefore, the change in free energy in swollen polyelectrolytes is defined by three components: mixing of pure solvent with the unstrained polymer network (free energy of mixing), conformational changes of the gel structure (elastic free energy), and the gradient of ions in the hydrogel and in the external solution (ionic free energy) [362]. The change in free energy $(\partial \Delta \mathrm{G} / \partial \mathrm{V})$ can be expressed in terms of the osmotic pressure $(\pi)$ at which the hydrogels are subjected in the swelling process as follows:

$$
-\left(\frac{\partial \Delta G}{\partial V}\right)_{n, T}=\pi=\pi_{m i x}+\pi_{e l}+\pi_{i o n} \quad \text { [Equation 4.1] }
$$

where, $\Pi_{m i x}, \Pi_{e}$ and $\pi_{i o n}$ are the osmotic pressure in the hydrogel due to the mixing with the solvent, changes in the configuration and the gradient of ions between the electrolyte and the polymer network, respectively.

At equilibrium swelling, $\pi$ in the equation is set equal to 0 and each term contributing to the osmotic pressure can be estimated: $\Pi_{\text {mix }}$ from the FloryHuggins theory, $\Pi_{e l}$ from the rubber-elasticity theory and $\pi_{i o n}$ by the Donnan equilibrium $[362,363]$. Therefore, the combination of all these terms, taking into account several assumptions, gives an expression as that described in Equation 3. 4 allowing the estimation of the molecular weight between crosslinking points.

Polysaccharide chains, as the case of $\mathrm{HA}$, are characterized by presenting interactions in aqueous media that are usually not taken into account when these equations are employed [366] including entanglements and hydrogen bonding. Additionally, hydrogels derived from polysaccharides are sensitive to the purification and drying processes behind the crosslinking reaction [221], leading to chains interactions and entanglements, which may alter the results obtained from these equations. The HA molecule shows a rigid structure in aqueous environments at neutral $\mathrm{pH}$ due to hydrogen bonding formation mediated by water molecules, and hence its hydrodynamic volume show high values giving a low motility of chains and solutions with very high viscosity, even at low concentrations [135]. However, the increment in $\mathrm{pH}$ of the media, such as the case of the alkaline solutions employed in the crosslinking solution, yields to a reduction of the hydrodynamic volume with a higher motility of chains, which is reflected in a dramatic reduction of the viscosity. This fact may not be explained only by the addition of ions and indicates that the rupture of hydrogen bonds due to the ionization of hydroxyls influences in such reduction of the hydrodynamic volume in the HA molecule. 
In order to discard the possible effect of entanglements on the swelling of the hydrogels, the HA-DVS polymer networks were obtained and purified by a determined process (described in section 2.2.1). Therefore, during the dissolution in $0.2 \mathrm{M} \mathrm{NaOH}$ and after the crosslinking reaction, the chains of hyaluronan remain contracted and hence forming entanglements. The volume increment observed in the samples in the posterior washing steps in the water/acetone mixture suggested that the water environment allow the rearrangement of chains in an extended fashion, whereas acetone limits the formation of hydrogen bonds in the molecules. The purification finished by washing steps in pure de-ionized water, and after that a soft drying at $40{ }^{\circ} \mathrm{C}$ with the aim to maintain the chains without producing entanglements. Taking into account all these considerations, the effect of the crosslinking agent, ionic strength and $\mathrm{pH}$ on the swelling process, and the determination of the crosslinking degree using the above-mentioned equations was addressed discarding chain entanglements. The higher HA:DVS molar ratios yielded a reduction in the equilibrium water content values in the hydrogels for all the media studied (Figure 3. 12), even for much higher DVS amounts that those for a theoretical degree of substitution of $100 \%$, suggesting the incorporation of additional crosslinks.

According to the swelling experiments, the effect of $\mathrm{pH}$ resulted in a higher water uptake for relative more alkaline media (Figure 3. 5, Figure 3. 6, Figure 3. 7 Figure 3. 12-A). Discrepancies between $\mathrm{pH}$ of 7 and 8 resulted not significant due to their similar values. However, the swelling of hydrogels in acidic medium, at $\mathrm{pH} 3$, resulted in lower swelling degrees. This fact can be explained by the degree of hydrolysis of the carboxylic acids within the HA disaccharides since these groups possess a pKa value around 3.2, and more acid $\mathrm{pH}$ values yield a lower degree of ionization decreasing chains repulsions. However, several studies have determined that at ionic strengths up to the physiological one (around 0.1 M) the charges within the HA molecule are shielded and the electrostatic effects on swelling resulted to be modest [135]. In this work, the effect of $\mathrm{pH}$ on the swelling of the hydrogels was performed at a constant ionic strength of $1 \mathrm{M}$, discarding changes in the swelling degree due to charge repulsions. Therefore, the higher swelling found at $\mathrm{pH}$ values of 7 and 8 is explained by the rupture of hydrogen bonds between HA chains directly bonded or mediated by water molecules, due to the ionization of hydroxyls within the HA and the increment of hydroxyl ions in the media. These results suggest that hydrogen bonds restricts the free swelling of the network acting in a similar way than the crosslinks. Moreover, the effect of the $\mathrm{pH}$ seems to not be influenced by the HA:DVS molar ratio (Figure 3.12-A), suggesting that hydrogen bonding is independent of the degree of crosslinking. 
On the other hand, the effect of ionic strength in the swelling of the HA-DVS polymer networks (Figure 3. 8, Figure 3. 9, Figure 3. 10 and Figure 3. $12 \mathrm{~B}$ ) is in agreement with the effect predicted by the Donnan equilibrium over the osmotic pressure due to ionic contributions:

$\Pi_{\text {ion }}=R T \cdot\left(\frac{i \cdot c_{2}}{z}-v \cdot\left(c_{s}^{*}-c_{s}\right)\right)$

[Equation 4.2]

where, $R$ is the gas constant, $T$ the temperature, $i$ the degree of ionization multiplied by the valence of the ionisable groups, $\mathrm{C}_{2}$ is the polymer concentration, $\mathrm{z}$ the valence of the ions in the polymer network, $\mathrm{Y}=\left(\mathrm{Y}_{+}+\mathrm{Y}-\right)$ the sum of the valences of the dissociating salt, $\mathrm{c}_{\mathrm{s}}^{*}$ and $\mathrm{c}_{\mathrm{s}}$ are the concentration of salt in the electrolyte and in the polymer network, respectively.

From the Equation 4.2 it can be deduced that the lower swelling degree in hydrogels immersed in media with higher ionic strengths is produced by the reduction of the osmotic pressure in the network. However, the equilibrium water content of hydrogels for ionic strengths of $1 \mathrm{M}$ and $2 \mathrm{M}$ resulted in no significant differences, suggesting that for relative high ionic strengths the effect on swelling is limited. Contrarily to the effect of $\mathrm{pH}$ on swelling, the ionic strength seems to have an effect dependent with the HA:DVS molar ratio: the lower the crosslinking agent, the lower the discrepancies in swelling degree. This observation may be related to the higher degree of crosslinking points within the polymer networks and subsequent reduction of the mesh size yielding to a restriction on swelling.

The effect of electrostatic repulsions on swelling can be estimated comparing the water uptake of the hydrogels at equilibrium swelling in water and in $0.15 \mathrm{M}$ PBS (Figure 3. 11 and Figure 3. 12-C). Therefore, the swelling degrees of the hydrogels in de-ionized water resulted in equilibrium water content values much higher than hydrogels immersed in 0.15 M PBS. This fact can be explained because of the absence of ionic species and a complete ionization of carboxylic acids in de-ionized water yielding to repulsions between chains. Additionally, in the inner lattices of the HA polymer networks are always present ionic species due to the ionization of carboxylic acids. This phenomenon produces a difference of osmotic pressure between the interior and exterior of the network, which is neutralized with ions from the external media. In the case of swelling in water, the absence of ions leads to greater increments of the water uptake to maintain the equilibrium conditions.

In the first instance, the diffusion of electrolyte in the HA-DVS polymer networks was supposed to follow a Fickian transport. This assumption implies that water 
diffusing in the hydrogels is dependent with the square root of time (Equation 3. 1) when the water uptake is less than the $60 \%$ of that in the equilibrium swelling. Moreover, this supposition was corroborated by the power law equation (Equation 3. 2) which determines the dependence of diffusion with time. Therefore, in general, the swelling exponents determined from this equation resulted in values close to 0.5 (Table 3. 3), for swellings ratios between $20 \%$ and $60 \%$ with respect to that at equilibrium, corroborating the Fickian diffusion. However, the diffusion process resulted to be lower for swellings less than $20 \%$, as can be observed in insets in Figure 3.5 to Figure 3. 11. This phenomenon is explained because the xerogels show a glassy state and the swelling process produces a transition from the glassy to a relaxed rubbery state, consequently, during the transition the water uptake is governed by the relaxation of the network. A similar effect is observed in hydrogels with higher contents of DVS, in which the swelling exponent increases, even values up 0.5 were found. Therefore, increasing the crosslinks, the water uptake shifts to a higher control due to the relaxation of the polymer chains. Surprisingly, the diffusion coefficient, which shows values between $4 \times 10^{-8}$ and $6.9 \times 10^{-7} \mathrm{~cm}^{2} / \mathrm{s}$, typical of HA hydrogels [367,368], results in higher values for compositions with larger amounts of DVS.

The degree of crosslinking in the HA-DVS polymer networks is usually estimated employing equations derived from the Flory-Rehner theory. However, many studies discard the contribution of ions in the osmotic pressure of the polymer networks [213]. From the results of swelling described above, it is derived that the polyelectrolyte nature of HA takes an important role in the swelling behaviour and must be considered. Therefore, in the present work, the crosslinking density was estimated considering all the terms contributing to the osmotic pressure within the networks (Equation 3. 4). On the other hand, these equations are commonly applied for hydrogels in which the posterior purification and drying processes are not taken into account [366]. In this work, the degree of crosslinking was determined from the HA-DVS polymer networks with the aim to discard false crosslinks related with chain entanglements. In any case, these equations were used under certain common hypothesis including the assumption of isotropic swelling, even though that is not the case of the HA-DVS polymer networks (Figure 3. 13 to Figure 3. 15). Taking into account all these considerations, calculations from these equations arise higher degree of crosslinking when higher DVS quantities are employed (Table 3. 5). Despite the DVS is introduced in molar excess in all the cases, the degree of crosslinking results in very low values $(14.6,17.7$ and $20.7 \%$ for HA:DVS molar ratios of 1:0.6, 1:0.9 and 1:1.5, respectively). Although this method overestimates the water content, due to the water retained in the cavities of the imperfect network, the degree of crosslinking is in agree with the determination of the molecular weight after enzymatic degradation (Figure 3. 53 and Table 3. 13). On the other hand, 
the determination of sulphur content, and hence the crosslinked degree calculated from the data of the elemental analysis yielded higher values (32.9, 53.3 and $87.2 \%$ for HA:DVS molar ratios of 1:0.6, $1: 0.9$ and 1:1.5, respectively) (Table 3. 7) than those predicted by the equations derived from the Flory-Rehner theory. Despite the fact that DVS is assumed to react principally by the primary hydroxyls, this method might overestimate the degree of crosslinking since some DVS might be included forming ineffective chains incorporated or interpenetrated in the bulk polymer networks. However, the possible ineffective DVS chains lack of double bonds since the infrared spectra of the HA-DVS polymer networks do not show these characteristic bands on the left of $3000 \mathrm{~cm}^{-1}$ (Figure 3. 3). Therefore, possible undesirable effects of vinyl groups in a biological environment are discarded. In any case, the low degree of crosslinking determined by both methods evidences the inefficacy of the reaction between HA and DVS.

The HA-DVS polymer networks show low mechanical properties, which are in agreement with similar studied hydrogels [73,238]. Additionally, the mechanical properties of the HA-DVS polymer networks can be tailored using different amounts of DVS, because increasing the DVS content produces a slight improvement of the mechanical properties of the hydrogels (Figure 3.16 to Figure 3. 19). Similar observations were found in crosslinked HA with DVS [369], as well as using other crosslinking methods $[73,238]$. The different HA-DVS polymer networks subjected under unconfined compression show two characteristic regions typical of hydrogels: the first one corresponds to the elastic region extending until strains around 0.2 , and the subsequent stage corresponds to the densification and collapse of the structure. The large elastic region appears because of the compression of lattices contained in the polymer networks and the displacement of water from themselves. The densification region is produced due to the collapse of the lattices, and hence the compression is applied in the bulk material containing lower amounts of water. Moreover, the elastic region is increased and the initial modulus takes lower values in hydrogels containing lower amounts of DVS, suggesting that the content of crosslinks increases the rigidity of the polymer networks. This fact is corroborated because of the higher contents of DVS yield to lower reached strains for a similar stress. On the other hand, the mechanical properties of the different HA-DVS polymer networks are clearly influenced by the swelling medium. These results are in agreement with the results from the swelling studies since, in general, the higher the water uptake, the lower the mechanical properties, due to the reduction in the contraction of the lattices formed between crosslinks.

As can be observed in the thermal degradation, after the purification and drying processes of the different HA-DVS polymer networks and the native HA, some water remains in the samples because of the lyophilisation process does not remove the bound water within the HA chains [370]. The water desorption 
process is produced until $180^{\circ} \mathrm{C}$, suggesting that the water is bonded to the HA chains. Moreover, the desorption process is slower in the native HA than in the HA-DVS polymer networks because native HA is in a lyophilized power form and the different HA-DVS polymer networks show a glassy contracted conformation giving a lower diffusion of water molecules. The addition of DVS does not produce significant changes in water content and degradation. On the other hand, the degradation of HA and HA-DVS chains starts at temperatures around $200{ }^{\circ} \mathrm{C}$ leading to similar residues contents at $700^{\circ} \mathrm{C}$, because of the major proportion in weight of HA with respect to DVS. However, the addition of DVS alters the maximal degradation rate, decreasing and displacing it to lower temperatures, due to the presence of a degradation step of self-polymerized DVS (pDVS) at lower temperatures than that corresponding to the HA. Contrarily, the maximum degradation rate between the HA-DVS polymer networks is reached faster with increasing the DVS content, suggesting a synergistic effect of the degradation stages in the pDVS sample and the HA-DVS polymer networks (inset in Figure 3. 21). 


\subsection{Crosslinked hyaluronan scaffolds}

The crosslinking of HA with different amounts of DVS allow the development of materials with soft tuneable mechanical properties maintaining high degrees of diffusion in aqueous environments. These properties together with the large clinical applications previously reported using these hydrogels encouraged us to consider the HA-DVS polymer networks as a good candidate in the development of scaffolds for brain tissue engineering. Moreover, the use of diverse DVS amounts suggests the possibility to obtain scaffolds with different mechanical properties and study this effect on cells from the CNS environment. HA scaffolds have been widely studied employing DVS and other crosslinking agents for soft tissue engineering, even central nervous system. The methods usually employed to develop HA based scaffolds are diverse including electrospinning [304], freeze-drying processes [278], particulate leaching [295]... One of the most important requisites in scaffold fabrication as artificial ECM for tissue engineering includes accomplishing an inner structure able to allow cell ingrowth, cell communication and diffusion of nutrients. In some cases, the techniques employed in developing scaffolds yield structures with small spaces for cell ingrowth and in many cases, the control over the size and interconnectivity of this inner structure shows a low reproducibility. In the present work, the development of HA-DVS scaffolds was carried out by a template technique with the aim to get a control over pore size and interconnectivity developing a repetitive method in the batch-to-batch production.

First of all, the optimal process in the fabrication of the PEMA templates (described in 2.2.2) was achieved by studying their microscopic morphology in order to stablish the more adequate conditions in the compression and sintering of PEMA beads. The conditions selected gave templates with not excessive deformation of the PEMA beads, maintaining an adequate interconnection between themselves and leaving space enough to the filling with the crosslinking solution (Figure 3. 22). In order to ensure the correct filling of the PEMA templates, the crosslinking solution was prepared with a lower HA concentration with respect to the HA-DVS polymer networks without no significant effect on the HA molecular weight after exposing to the alkaline medium (Figure 3. 23). The correct crosslinking of the HA with DVS was suggested by the integrity of the materials observed during the purification process and corroborated by the posterior analysis of the dry HA-DVS scaffolds by SEM (Figure 3. 27). Additionally, different properties on PEMA templates and HA-DVS scaffolds were evaluated with the aim to ensure repetitively in the batch-to-batch production. These parameters and studies include the determination of the thickness in PEMA templates between the compression and sintering stages, the analysis of the porosity in the interstices of the templates, thermogravimetric analysis of the HA-DVS scaffolds to ensure the elimination of residual PEMA (Figure 3. 24), 
microscopic imaging by SEM and compression tests on the final scaffolds (described in the following). These parameters and studies resulted to be similar between scaffolds obtained in different batches, and hence the fabrication of the HA-DVS scaffolds was considered repetitive.

In addition to the observed integrity of the HA-DVS scaffolds during the fabrication and purification processes, the effectiveness of the crosslinking was achieved from the IR spectra (Figure 3. 26) and posterior elemental analysis (Table 3. 8). The infrared spectra of the different HA-DVS scaffolds compared with that of native HA showed sulfone groups, and the posterior elemental analysis confirms the presence of sulphur content. With respect to the infrared spectra, increasing DVS content resulted in a higher absorption band attributed to the symmetric stretching of sulfone groups (at wavenumber of $1080 \mathrm{~cm}^{-1}$ ). The band corresponding to the asymmetric stretching of sulfone groups (wavenumber of $1290 \mathrm{~cm}^{-1}$ ) is presented but its relation with the DVS amount is not clearly observed. Moreover, the effective crosslinking between HA and DVS is corroborated comparing the relative intensity of the peaks at $1100 \mathrm{~cm}^{-1}$ (stretching of the ether groups) and at $1600 \mathrm{~cm}^{-1}$ of the different HA-DVS scaffolds and the native HA. Therefore, the intensity of the band corresponding to the ether groups is more intense in the HA-DVS scaffolds than in the native $\mathrm{HA}$, corroborating the ether formation and hence the adequate crosslinking of $\mathrm{HA}$ with DVS. On the other hand, the determination of the degree of crosslinking derived from the elemental analysis yielded lower values $(18.4,20.9$ and 42.6 for HA:DVS molar ratios of $1: 0.6,1: 0.9$ and $1: 1.5$, respectively) than those corresponding to the solv_HA-DVS polymer networks. This fact suggests that the effectiveness of the crosslinking between the interstices of the PEMA templates is altered.

The morphological characterization of the different HA-DVS scaffolds (Figure 3. 27) and the thermogravimetric analysis (Figure 3. 25) resulted in no evidences of PEMA residues. The content of DVS seems not to alter the morphology of the scaffolds, which resulted in an interconnected porous inner structure where the HA walls form a sheet-like structure typical of scaffolds based on polysaccharides [279]. The interconnections in the inner surface resulted in sizes ranging from 60 to $100 \mu \mathrm{m}$, suggesting their adequate dimensions to permit cell ingrowth [371]. The porosity due to the sintered beads was estimated to have a maximum value of $75 \%$; however, the porosity determined by gravimetric measurements resulted in higher values, around $99 \%$. This fact is explained because HA chains are contracted during the washing process in acetone, the subsequent swelling and freeze process can produce additional pores in the size range of those to the PEMA template itself, due to the growth of ice nuclei during the freeze process [278]. The different HA-DVS scaffolds showed similar porosity taking into account the effect of small and large pores together and only the large pores, suggesting 
the lack of micro- and nano-porosity due to the removal of solvent (Figure 3. 28). Additionally, the walls of the inner surface of HA-DVS scaffolds observed by SEM (Figure 3. 27) showed a bulk material with absence of micro- and nano- porosity. This closed structure of the bulk material suggests that the drastic immersion of the crosslinked $\mathrm{HA}$ in acetone yields to a contraction of the molecules suggesting stable entanglements between chains.

In order to compare the properties of HA-DVS scaffolds with HA in bulk, HA-DVS polymer networks (WI_HA-DVS samples) were developed and purified using similar conditions than those of the HA-DVS scaffolds. Despite the differences in HA concentration in the crosslinking solution, the much lower swelling degree on WI_HA-DVS samples (inset in Figure 3. 29) than on solv_HA:DVS samples (Figure 3.12 C) corroborated the influence of post-treatments in the final swelling of the hydrogel. Therefore, the establishment of interactions and entanglements between HA chains due to the drastic transition of hydrogels from a conformation containing contracted and entangled chains (after the crosslinking reaction using alkaline medium) to a non-aqueous environment (such as the case of acetone immersion) was corroborated. In spite of the reduction in the swelling degree observed in the polymer networks with higher contents of DVS, the equilibrium water content values of the WI_HA-DVS samples immersed in water and $0.15 \mathrm{M}$ PBS evidence no significant differences, suggesting an important influence of the entanglements in their swelling behaviour. On the other hand, the HA-DVS scaffolds resulted in a much higher water uptake compared with the lyophilized WI_HA-DVS samples and differences between media and compositions resulted to be not significant when the salt weight is taken into account. The much higher water uptake by the HA-DVS scaffolds respect to the non-structured hydrogels can be explained because of its high porosity (around $99 \%$ ). However, taking into account the porosity and determining the water uptake by the bulk HA contained in the HA-DVS scaffolds, the values of EWC resulted much lower than in the WI_HA-DVS samples, suggesting that the material structure have an important effect on swelling.

The principal advantage of employing hydrogels against other polymeric systems is their similar mechanical properties with respect to brain tissue [73]. Moreover, several works have demonstrated that hydrogels with closer mechanical properties to those of the brain tissue favour the differentiation of NPCs to neural lineages against glial ones, and enhance neurite extension in neural cell lines [75]. For this reason, the different HA-DVS scaffolds were conducted to compression tests and the parameters derived from them were compared with those of the brain tissue. The HA-DVS scaffolds under compression showed a typical behaviour of hydrated hydrogels with open-mesh structures, resulting in a very large first elastic region and after densification zone a second elastic region at advanced strains, above values of 0.7 (Figure 3. 30) [280]. The large elastic 
region is due to the compression of the lattices and the effect of water displacement from the inner hydrogel, while in the densification region the compression is applied in the bulk HA, containing some remaining water, due to the collapse of the pores and displacement of water from HA. The content of DVS on the HA-DVS scaffolds yielded appreciable changes, which become more evident for the scaff_HA:DVS 1:1.5 samples, indicating an effect of the degree of crosslinking on the mechanical properties of the scaffolds. The module and final strain (Figure 3. 31) determined from the compression tests corroborated that the higher amounts of DVS increase their rigidity, since the scaff_HA:DVS 1:1.5 samples resulted in higher initial modulus values and lower final strains indicating a less soft behaviour. However, the different compositions showed similar values in the parameters obtained from the compression tests, and the scaffolds are considered such as soft materials. Moreover, the result of the comparation of HADVS scaffolds with brain tissue is that both have similar mechanical properties, suggesting that HA-DVS scaffolds are good candidates for brain tissue engineering from the point of view of their mechanical properties. As it was expected, bulk materials corresponding to the WI_HA-DVS samples resulted in higher mechanical properties than HA-DVS scaffolds. These materials showed a shorter elastic region and the densification was more pronounced, and it appeared at lower strains. This fact is explained by the lack of pores within WI_HA-DVS samples.

Such as other HA-DVS based materials, the HA-DVS scaffolds resulted in a similar thermal degradation, showing a first stage associated to the desorption of water and a principal degradation step starting at $200{ }^{\circ} \mathrm{C}$ and extending through all the temperature range (Figure 3. 32 and Figure 3. 33). Despite the similar behaviour of HA-DVS scaffolds and native HA submitted to thermal degradation, some differences were appreciated. As can be observed in the first stage of weight loss, the HA-DVS scaffolds gave higher amounts of bound water; such fact can be explained by the increment of specific surface because of their porosity. Moreover, the principal degradation stage yielded lower degradation rates and percentage of residues at $700{ }^{\circ} \mathrm{C}$ than native $\mathrm{HA}$, suggesting that the DVS content reduces, both degradation rate and residual content, which is in agreement with the thermal degradation observed in the thermograms of pDVS (Figure 3. 20 and inset in Figure 3.21). 


\subsection{Poly(MeHA-co-EA) copolymer networks}

Previous studies have reported that the HA based biomaterials, and particularly the DVS crosslinked ones, possess low resistance against the degradation mechanisms found at physiological conditions [222,372]. In order to obtain hydrogels with longer residence times and tuneable properties, poly(MeHA-coEA) copolymer networks were developed. The incorporation of ethyl acrylate in the polymer networks was performed with the aim to obtain semi-biodegradable block copolymers, providing the presence of artificial ECM through the regenerative process. Moreover, the incorporation of EA allows to modulate several properties within the hydrogels including mechanical properties and swelling degree.

Poly(methacrylates) and poly(acrylates) have been widely employed and investigated for a wide range of tissue engineering applications, even some of them have found clinical applications [349]. In particular, poly(ethyl acrylate) networks constitutes a less explored system for tissue engineering applications, but few studies have demonstrated their good biological properties, in terms of cell colonization, including good biocompatibility with the brain tissue even showing infiltration of neural and glial cells [49]. Moreover, the poly(ethyl acrylate) networks show softer mechanical properties compared with other poly(methacrylates) and poly(acrylates), suggesting it as a more adequate polymer for soft tissue engineering. Additionally, the polymerized ethyl acrylate is a relatively hydrophophic material [353], suggesting that the use of different amounts of ethyl acrylate in the copolymerization with HA can be useful in modulating the swelling degree of the resulting hydrogels.

However, the copolymerization of ethyl acrylate cannot be successfully performed directly with native HA, and it needs to be previously chemically modified. Despite the wide range of possible chemical modifications within the HA molecule, in the present work, the HA has been esterified using anhydride methacrylic giving methacrylate hyaluronan (MeHA) (see Figure 1. 7-A). This chemistry incorporates methacrylate groups, mainly by the primary hydroxyls of the $\mathrm{N}$-acetyl glucosamine subunit within the HA. Therefore, this HA derivative possesses vinyl groups, which are able to carry out polymerization reactions, and hence the copolymerization with ethyl acrylate (see Figure 1.7-B). This chemistry has been employed by other authors with the aim to copolymerize HA and other synthetic polymers and functionalize HA chains [241].

The HA derivative, MeHA, has been previously synthesized in both, aqueous and organic media [373]. However, the reaction in an aqueous medium possesses a difficult control, because the $\mathrm{pH}$ must be maintained at higher values with the aim to avoid the formation of methacrylic acid, which reduces the degree of methacrylation. This fact together the inherent properties of HA in solution, high 
viscosity and molecular hindrance, and the phase separation between aqueous medium and anhydride methacrylic produce in general low methacrylation degrees. In order to provide an adequate degree of methacrylation, the reaction was carried out employing a $\mathrm{HA}$ solution in $0.2 \mathrm{M} \mathrm{NaOH}$ with the aim to reduce $\mathrm{HA}$ viscosity and ensure high $\mathrm{pH}$ values avoiding excessive methacrylic acid formation. Moreover, the anhydride methacrylic reagent was added dropwise in excess with the aim to obtain an adequate degree of functionalization and reduce phase separation, as suggested in previous works [73,238]. After the reaction time, the $\mathrm{pH}$ falls down to values of 3 indicating the inevitable formation of methacrylic acid as by-product. However, after the purification processes, the $\mathrm{pH}$ taken values around neutrality, even the analysis of the macromer by chromatography, infrared spectroscopy and nuclear magnetic resonance showed non-remaining methacrylic acid. However, as in the case of WI_HA-DVS samples, the precipitation of MeHA in acetone during the purification steps yielded chain entanglements, which effect can be observed by the decrease of the solubility in water. Additionally, the posterior analysis of MeHA macromer by chromatography resulted in weight average molecular weights around 2.5 $\mathrm{MDa}$, much higher than the native HA (Figure 3. 34), which is only explained by the formation of entanglements.

On the other hand, the functionalization of HA chains was addressed by Fourier transformed infrared spectroscopy and nuclear magnetic resonance. The MeHA spectra obtained by FTIR did not show the characteristic bands of vinyl groups because of the presence of broad bands corresponding to the associated $\mathrm{O}-\mathrm{H}$ stretching of carboxylic acids, hydroxyls and associated $\mathrm{N}-\mathrm{H}$ bonds of the amide groups (Figure 3. 35). However, comparing the spectra of native HA and MeHA, the band around $1000 \mathrm{~cm}^{-1}$ increased its relative intensity with respect to peaks associated with unmodified groups ( $\mathrm{N}-\mathrm{H}$ stretching), suggesting an increment of the $\mathrm{C}-\mathrm{O}-\mathrm{C}$ stretching and hence the formation of ester groups. Additionally, the posterior nuclear magnetic resonance of purified MeHA showed peaks at 6.1, 5.6 and $1.85 \mathrm{ppm}$ (Figure 3.36) indicating the presence of methacrylate groups as previous works $[73,212]$. Therefore, from the ${ }^{1} \mathrm{H}-\mathrm{NMR}$ spectrum of MeHA, the degree of methacrylation was determined, resulting to be a value of $12.5 \%$, which is comparable with that obtained by other authors employing similar conditions [238].

Once the functionalization of HA was determined, the MeHA was copolymerized with different amounts of ethyl acrylate with the aim to obtain semi-biodegradable copolymers with tuneable properties and resistance against enzymatic degradation. The amounts of EA employed in the copolymerization were chosen in agreement with the solubility of EA itself in the reacting solution employed. Therefore, the reaction was performed in a solvent mixture of $0.15 \mathrm{M}$ PBS and DMF using a low concentration of MeHA because of the high viscosity of MeHA 
solutions and the entanglements between chains. On the one hand, the use of $0.15 \mathrm{M}$ PBS reduces the viscosity of MeHA and, on the other hand, the use of DMF increases the solubility of EA and reduces the entanglements between MeHA chains, due to its intrinsic nature as strong hydrogen bonding acceptor decreasing the interactions between chains [374,375]. Additionally, the incorporation of DMF increases the solubility of ethyl acrylate, which shows a reduced solubility in aqueous medium. On the other hand, the incorporation of crosslinking agent, $\mathrm{N}, \mathrm{N}$-methylenebisacrylamide, was aimed to ensure the crosslinking between methacrylate groups within the MeHA and/or the ethyl acrylate networks, since the high viscosity and the low degree of methacrylation of MeHA poses a handicap to obtain an adequate poly(MeHA-co-EA) copolymer network. Therefore, during the mixture of all the reagents (monomer, macromer, crosslinking agent and initiator) in the polymerization solution, phase separation and MeHA entanglement was not observed for the different compositions.

Despite the relative low degree of methacrylation obtained in the production of $\mathrm{MeHA}$, its copolymerization with EA employing MBAA as crosslinking agent was accomplished for all the compositions. After the copolymerization reaction, the different poly(MeHA-co-EA) copolymer networks resulted to be continuous and soft samples, without observing segregation of material during the purification processes. However, the copolymer networks with larger amounts of EA (above MeHA:EA weight ratios of 1:2) resulted to have an opaque aspect with a lower consistence, suggesting some phase separation. This fact can be explained because of the largest MeHA:EA weight ratios (1:5 and 1:10) are in the limit of phase separation for the mixture of solvents employed in the copolymerization reaction. Moreover, during the copolymerization reaction, the new forming networks (the growing chains of polymerized EA and MeHA) decreases their solubility in the solvent mixture.

The copolymerization of both components and their presence in the different copolymer networks was addressed by FTIR. The spectra of the copolymer networks showed the characteristic absorption bands of PEA and MeHA homopolymer networks. Therefore, the copolymer networks with higher content of MeHA gave a more intense band at $1000 \mathrm{~cm}^{-1}$ due to the major presence of CO-C groups within the MeHA molecule with respect to the EA one. In this way, the broad band from 3600 to $2500 \mathrm{~cm}^{-1}$, attributed to associated $\mathrm{N}-\mathrm{H}$ and $\mathrm{O}-\mathrm{H}$ stretching vibrations, resulted more intense and narrow in samples containing MeHA because this molecule contains larger amounts of these groups and includes bound water. The copolymer networks show multiple peaks in the range between $1800 \mathrm{~cm}^{-1}$ and $1600 \mathrm{~cm}^{-1}$, associated with a unique vibration, the stretching of $\mathrm{C}=\mathrm{O}$, whose intensity depends on the amount of $\mathrm{EA}$ or MeHA employed in the copolymerization reaction. The band at $1735 \mathrm{~cm}^{-1}$ in the PEA homopolymer and the different copolymer networks, corresponding to free $\mathrm{C}=\mathrm{O}$ 
groups, appears at the same wavenumber suggesting that PEA and MeHA chains lack of hydrogen interactions at the molecular level. However, the use of MBAA could slightly increase the interaction between the PEA and MeHA networks since the characteristic bands of associated carbonyls in Amide I and carboxylates within the MeHA and MBAA molecules were displayed as overlapped bands around $1600 \mathrm{~cm}^{-1}$. On the other hand, the IR spectra suggest that the PEA chains are forming a network without hydrogen bonding association, whereas the MeHA and MBBA chains in the copolymer networks may form interactions by hydrogen bonds. These observations suggest the lack of interactions between MeHA and PEA networks at the molecular level induced by hydrogen bonds. However, the incorporation of MBAA may slightly increase the interaction between both components due to hydrogen bonding between the crosslinking agent and the MeHA molecules, and hence this fact could indicate a low miscibility between PEA and MeHA networks mediated by MBBA.

The copolymerization yields copolymer networks with a hydrogel-like behaviour for all the compositions studied. Therefore, all the compositions showed a high water uptake under immersion in $0.15 \mathrm{M}$ PBS, even considering that PEA homopolymer networks are relatively hydrophophic (Figure 3. 38). However, the swelling degree is drastically reduced when MeHA homopolymer networks are compared with the copolymer ones, even in those in which low amounts of EA were employed. Therefore, the MeHA homopolymer networks show a very high equilibrium water content (values around 100); while in the copolymer networks using EA weight fractions of 0.2 this value is drastically reduced (values around 50). Moreover, the incorporation of higher amounts of EA produces a continuous reduction on the swelling degree until an apparent stabilization. However, the $E W C$ reached values slightly higher than that characteristic of PEA homopolymer networks (EWC values below 0.1) [353]. This fact may be explained because of the high water uptake of the MeHA component and the additional porosity produced in the freeze-drying process, and hence water could be incorporated in the inner porous structure.

On the other hand, the copolymerization including greater contents of EA yielded hydrogels with less soft mechanical properties, including the copolymers networks containing highest EA contents, which are considered as not consistent ones (Figure 3. 39). The different copolymers showed soft mechanical properties when compressed, displaying the typical behaviour of hydrogels open-lattice structures. The copolymer networks display a large elastic region and, after a transition region, they undergo a densification, and a second elastic region is observed where the compression is applied over the bulk material due to the displacement of water and the collapse of pores. The first elastic region is characterized by a high strain rate when the applied stress is small, while the second one is characterized by a limited change in strain increasing the applied 
stress. However, the first elastic region becomes narrower with increasing the EA content in the copolymer networks, and hence the densification region is reached at lower strains. Therefore, this behaviour in copolymer networks with higher amounts of EA is translated to the parameters determined from the stress-strain curves: the initial elastic modulus take higher values, the final strain is slightly lower and the final modulus decreases (Figure 3. 40). However, the stress-strain curves and the initial modulus, reaching values between 0.5 and $2.5 \mathrm{kPa}$, display a behaviour typical of hydrogels, even the copolymer networks with higher content of EA (weight fractions above 0.9 ) resulted in a very soft behaviour. This fact suggests discrepancies in the formation of the PEA networks due to the possible low solubility during the copolymerization reaction, formation of internal porosity during the freeze-drying process and a predominance of MeHA networks over the mechanical properties of the copolymer networks. Despite the differences in the synthesis and purification of MeHA homopolymer networks with respect other authors, their soft mechanical properties are in agreement with previous works $[73,238]$.

The macroscopic images of the cross-sections in the different copolymer networks (Figure 3. 41) evidence changes in the inner structure by varying the composition of EA respect to MeHA. Therefore, the MeHA homopolymer displayed pores with a sheet shape, whereas lower amounts of EA produces inner structures with spherical pores. However, copolymers with high contents of EA lead to the collapse of the internal pores giving disordered internal structures. Therefore, the swelling degree and compression of the copolymers networks are clearly influenced by the presence of this internal structure. Moreover, from the macroscopic study arises that the copolymer networks may be obtained with tuneable topography varying the EA content in the copolymerization reaction. Besides, the copolymerization of MeHA and EA can be carried out into the interstices of the PEMA templates, in a similar way that the process described in section 2.2.2. In this process, it is possible to obtain scaffolds based on poly(MeHA-co-EA) copolymer networks, and hence a method to develop scaffolds with a controlled porosity and pore size (Figure 4. 1). These scaffolds display interconnected pores with an average diameter of $204.4 \pm 51.8 \mu \mathrm{m}$ and interconnections with a diameter of $78.7 \pm 20.3 \mu \mathrm{m}$. 

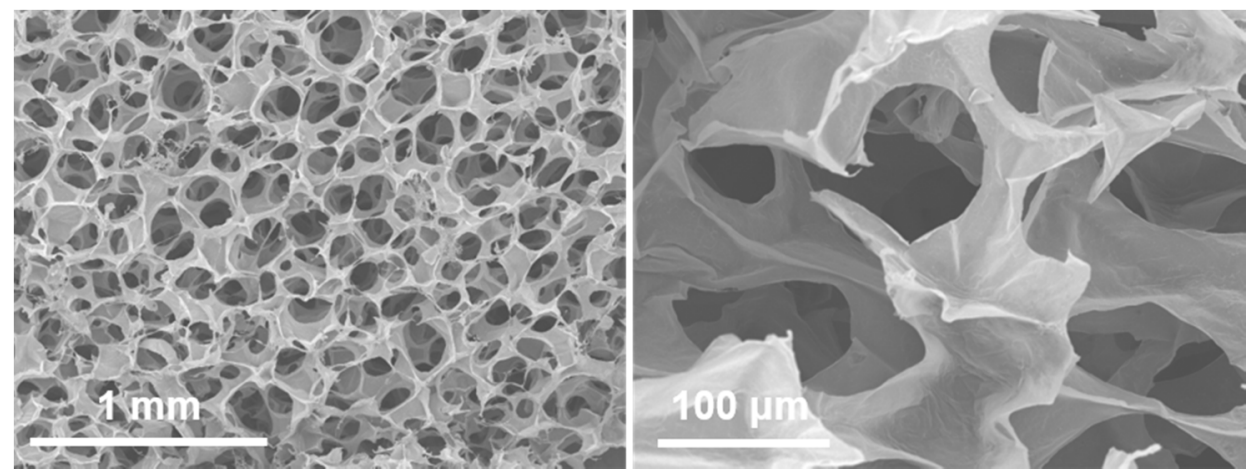

Figure 4. 1. Cross-section in the scaffolds obtained from the MeHA:PEA 1:10 composition.

The methacrylation of $\mathrm{HA}$ and subsequent crosslinking using MBAA did not significantly alter the thermal degradation of the HA derivative with respect to the native $\mathrm{HA}$, as is observed comparing the thermograms of native HA (Figure 3. 20) and MeHA homopolymer networks (Figure 3. 42). This fact can be explained by the low degree of functionalization and the big size of the HA chains and its disaccharide unit, and hence the MeHA molecules are considered as a big one. Moreover, the MeHA homopolymer networks resulted to content similar bound water than other developed HA-DVS based materials, indicating a low influence of the employed crosslinking agents and modification in the ability of MeHA to associate with water molecules. On the other hand, the incorporation of higher contents of EA yielded to a drastic reduction of bound water because of the relative more hydrophobic domains of the EA molecule. In addition to the stage of water desorption, the thermograms of the poly(MeHA-co-EA) copolymer networks showed two differentiated degradation stages corresponding to each component. This behaviour under thermal degradation is typical of block copolymers [376]. However, a possible low interaction between both components in the copolymer networks cannot be achieved by thermogravimetry because of the degradation of MeHA continues in the whole temperature range studied altering that corresponding to the PEA phase (inset in Figure 3. $42 \mathrm{~B}$ ).

In order to determine the interaction between both components, MeHA and PEA, the different copolymer networks were characterized by differential scanning calorimetry. The thermograms of the copolymer networks showed a vitreous transition associated to the amorphous PEA phase (Figure 3. 43 and Figure 3. 44). However, the vitreous transition in the PEA homopolymer networks occurs at higher temperatures than in the copolymer networks. Moreover, the temperature of glass transition was independent of the EA content in the different copolymer networks, suggesting that PEA and MeHA phases interact at least in some degree. After desorption of bound water, the temperatures of glass 
transition took relative higher values than those in samples containing bound water. However, the glass transition in PEA homopolymer networks was displayed at higher temperatures than those of the copolymer networks, corroborating that MeHA network interacts with the PEA one, and hence some degree of interaction occurs between both phases. Additionally, the lack of displacement of the vitreous transition between the different copolymer networks implies that MeHA does not have an influence on the thermal properties of the PEA network.

Due to the conditions employed in the copolymerization reaction, a quantity of the EA employed was expected to not being involved in the formation of the PEA network. Taking into account this hypothesis, the real PEA content in the different copolymer networks, considering the bound water as part of the MeHA homopolymer network, was determined by different methods: from calorimetry of both dried and water-containing samples (Table 3.11), and from the residues determined from the thermogravimetric analysis (Table 3. 10).

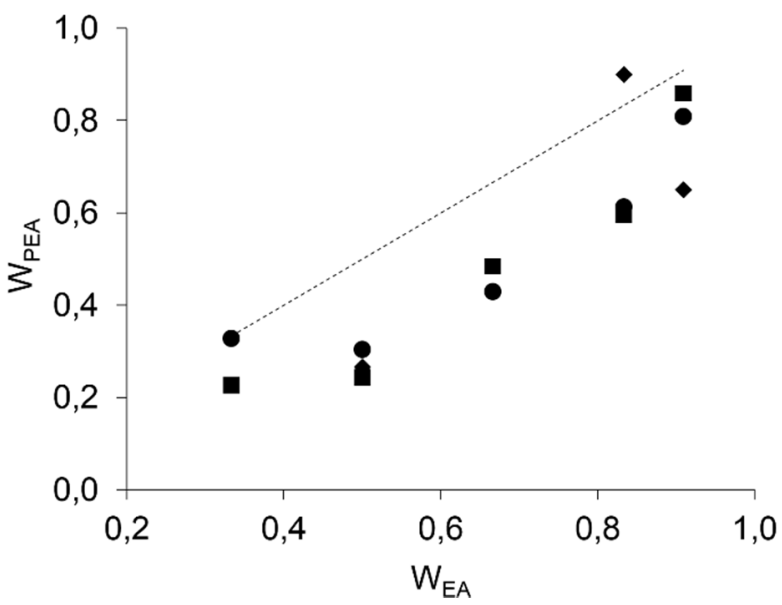

Figure 4. 2. $W_{P E A}$ in each copolymer network as function of $W_{E A}$ employed in the copolymerization reaction. The weight fraction was determined from the DSC thermograms in networks containing bound water (squares), from the DSC thermograms after drying the samples (circles) and from the residues determined by TGA (diamonds). Dotted line represents the theoretical $W_{\text {PEA }}$ in each copolymer network.

Therefore, the experimental PEA content resulted in lower values than expected, considering that the total amount of EA forms efficient networks (Figure 4. 2). The real PEA content and the expected one resulted in more discrepant values for intermediate compositions, suggesting that MeHA could have an effect on the formation of the PEA network for such compositions. The PEA content for each 
composition resulted, generally, in similar values independently of the method employed in its determination, and hence its value was supposed the average of those calculated by each method (Table 4. 1). Additionally, the similarity of the calculated PEA content, independently of the method used (from TGA and DSC analysis), asserts that the change in thermal properties of the copolymer networks is exclusively due to the amorphous PEA network.

Table 4. 1. Weight fraction of EA employed in the copolymerization reaction and the real weight fraction of PEA in each copolymer network.

\begin{tabular}{cccccc}
\hline W $_{\text {EA }}$ & 0.33 & 0.50 & 0.67 & 0.83 & 0.91 \\
\hline W & & & & & \\
\hline
\end{tabular}

The copolymer networks were measured by dynamic analysis at $1 \mathrm{~Hz}$ in the temperature range between $-60{ }^{\circ} \mathrm{C}$ and $60{ }^{\circ} \mathrm{C}$ and showed a main, $\alpha$, relaxation process between $-30 \stackrel{\circ}{\circ}$ and $50 \stackrel{\circ}{\circ}$ (Figure 3. 45). A drastic drop in the elastic modulus characterizes the transition from the glassy to the rubbery state derived from the amorphous PEA component. The intensity on the reduction of the elastic modulus resulted to be dependent with the weight fraction of PEA contained in the copolymer network. Moreover, this transition was observed in the $\tan \delta$ thermograms, which contain a maximum indicating the loss of mechanical properties around the $a$ relaxation process. However, the copolymers synthesized with lower EA weight fractions ( $\mathrm{W}_{\mathrm{EA}}$ of 0.33 ) do not register this transition stage, suggesting that the PEA matrix does not have an influence on their mechanical properties, and hence the PEA may be presented as a noncontinuous phase for this composition forming aggregates or isolated chains included or linked to a continuous matrix of MeHA. The PEA homopolymer networks attained the higher elastic modulus in the glassy state with values around $2 \mathrm{GPa}$, which are typical of PEA in such state [377]. However, the different copolymer networks displayed lower values of elastic modulus in this region with increasing the MeHA fraction, suggesting that in the glassy state the PEA network governs the mechanical properties. After the $\alpha$ relaxation process, the elastic modulus of the PEA homopolymer network is drastically reduced to values around $0.4 \mathrm{MPa}$ at $50{ }^{\circ} \mathrm{C}$, as expected for PEA networks [377]. However, the different copolymer networks and the MeHA homopolymer networks attained similar values in the rubbery state, between 10 and $15 \mathrm{MPa}$, but the rate between the modulus at the rubbery and the glassy state, $E^{\prime} / E_{-60}^{\prime}{ }^{\circ} C$, resulted to be dependent with the EA content showing higher values for lower EA contents. This observation suggests that after the main relaxation process, the copolymer networks have a more similar behaviour with the MeHA homopolymer networks, but the EA content decreases the mechanical properties of the copolymer 
networks. The copolymerization of EA with MeHA produces a displacement of the $\alpha$ relaxation to lower temperatures compared with the PEA homopolymer network, as can be observed in the thermograms of $E^{\prime} / E_{-60^{\circ} \mathrm{C}}$ and $\tan \delta$, indicating some interaction between MeHA and PEA chains. However, as in the case of the DSC thermograms, this $\alpha$ relaxation is not displaced with the EA content in the different copolymer networks indicating that the interaction between both kinds of networks is in any case minimal. This minimal interaction may be due to the reaction during the polymerization process of methacrylate groups of MeHA with the crosslinking agent (MBAA) and PEA chains. On the other hand, the $E^{\prime} / E^{\prime}-60^{\circ} \mathrm{C}$ and $\tan \delta$ thermograms do not display secondary transitions associated with PEA chains, observing a stabilization of both, $E^{\prime} / E_{-60}^{\prime} c$ and $\tan \delta$, for temperatures above the $\alpha$ relaxation. However, the $\tan \delta$ thermograms of the copolymer and MeHA homopolymer networks showed an increment of losing mechanical properties that can be attributed to the desorption of water at temperatures above $20 \stackrel{\circ}{ } \mathrm{C}$. The results of the dynamic-mechanical thermograms displayed that the mechanical response of copolymers in the dry state is influenced by the PEA content at physiological temperatures. This observation is in consonance with those results reported in the compression tests, where the mechanical properties of the swollen copolymer networks resulted to be dependent with the PEA weight fraction.

Due to the nature of MeHA, the procedure employed in the copolymerization and the results showed above, the MeHA homopolymer network is considered to be as a continuous phase, at least in compositions with lower amounts of EA. On the other hand, the growing PEA network during the copolymerization is supposed to be associated in some degree with the MeHA one, but the phase distribution of the PEA networks for each copolymer composition is not well understood. In order to address the phase distribution, the logarithmic value at $60{ }^{\circ} \mathrm{C}$ of the ratio between $E^{\prime}$ at each temperature and at $-60{ }^{\circ} \mathrm{C}$ in the different poly(MeHA-co-EA) copolymer networks was fitted to a Takayanagi's model for bi-phasic systems [378], considering disperse phase both components, MeHA and PEA, respectively. 


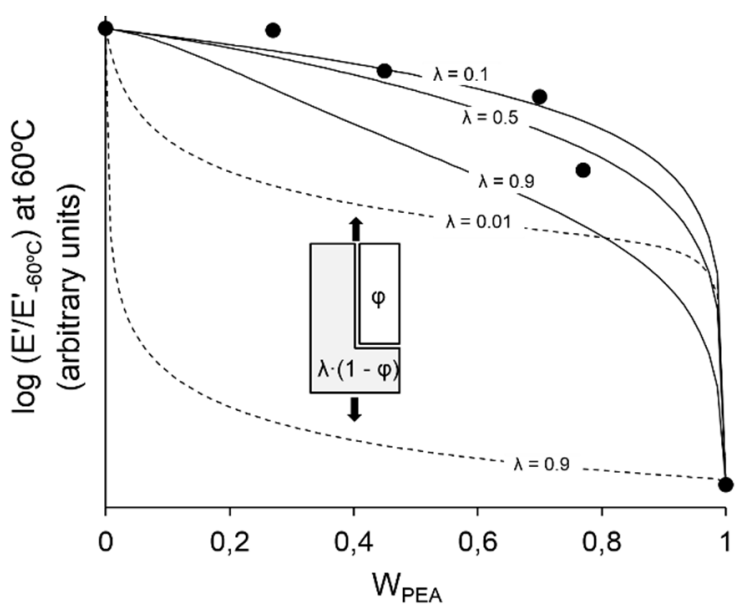

Figure 4. 3. Fitting of the experimental $E^{\prime}$ values from the dynamical-mechanical assay to the Takayanagi's model for bi-phasic systems, taking the data as the absolute value of the logarithmic value at $60^{\circ} \mathrm{C}$ of the ratio between $\mathrm{E}^{\prime}$ at each temperature and at $-60^{\circ} \mathrm{C}$ in the different poly(MeHAco-EA) copolymer networks. The approximation to the Takayanagi's model considering the PEA (solid lines) and MeHA (dashed lines) as the components in the disperse phase, respectively, with different $\lambda$ values are fitted. Inset shows the Takayanagi's block model in series.

Figure 4.3 reveals that the elastic modulus at $60{ }^{\circ} \mathrm{C}$ in the copolymer networks may be predicted by the Takayanagi's model. The data fitted to the Takayanagi's block model, considering as disperse phase both components, arise similar conclusions: the $\lambda$ fraction in the different copolymer and homopolymer networks resulted in small values, and it was slightly increased in the sample with the largest PEA weight fraction (WPEA of 0.77). These observations indicate that disperse and continuous phases within the copolymer networks are disposed in a parallel arrangement. However, the copolymer network with the lowest WPEA, 0.27 , may consist in a continuous MeHA phase including isolated PEA chains, since it is characterized by a modulus similar to the MeHA homopolymer network and some deviation from the values predicted by the model. Additionally, the experimental elastic modulus of the copolymer network with the largest WPEA, 0.77 , is slightly higher than the predicted for a bi-phasic system without $\lambda$ fraction suggesting the formation of aggregates.

Taking into account the different analysis above described, the copolymer networks are composed of two different phases, which coincide with each component, and owing a low interaction degree at the molecular level. Moreover, the copolymers showed mechanical properties more similar to the MeHA homopolymer networks indicating that MeHA governs the properties of the copolymer networks. However, the intermediate copolymers contain a parallel arrangement of the phase structure indicating co-continuity, except for the 
extreme compositions: high MeHA weight fractions lead to isolated PEA chains and the composition with larger PEA content slightly turns the co-continuity of phases to an aggregate-like structure. 


\subsection{Enzymatic degradation}

In the biological environment, there are multiple factors involved in degrading HA: free radicals, hydrolysis mediated by water molecules and enzymes. However, the most prevalent degradation mechanism of HA in the biological environment is due to the enzymatic degradation catalysed by hyaluronidases $[145,153]$. Moreover, the enzymatic activity of hyaluronidases is increased because their synthesis is up-regulated in certain pathologies, including the CNS ones, yielding higher concentrations of enzyme in the physiological fluids, and hence the degradation of HA chains is faster [154].

The study of the enzymatic degradation induced by hyaluronidase activity was performed with the aim of mimicking the biological environment, understanding that in the biological environment, and in particular in the damaged brain tissue, the degradation of $\mathrm{HA}$ is mediated by multiple factors, which synergistic effect cannot be taking into account in an in vitro model. Taking into account this limitation, the study of the enzymatic degradation in the HA-based materials is aimed to estimate the residence time and integrity of the materials when subjected to degradation. Moreover, the biodegradation of HA chains is an important matter to promote certain biological functions. Therefore, the HA fragments containing between 4 and 25 disaccharides up-regulate important biological functions in the regenerative process, including angiogenesis and cell migration and proliferation [190]. For this reason, in addition to the characterization of the materials subjected to enzymatic degradation, the byproducts derived from the digestion were analysed by chromatography with the aim to determine the size of the fragments, and hence the biological functions upregulated by themselves may be estimated.

The enzymatic degradation of HA by the activity of mammal hyaluronidases, as that employed in the present work, possess a well-known mechanism of action [157]. Therefore, the enzymes randomly hydrolyse HA chains cleaving the $\beta(1-4)$ glyosidic linkage between disaccharide units. However, this reaction is initiated by the interaction of the enzyme with the $\mathrm{N}$-acetyl glucosamine moiety and the oxygen forming the $\beta(1-4)$ glyosidic bond. This specific mechanism suggests that the degradation of $\mathrm{HA}$ chains composing the different materials may be affected by their own conformation. In this sense, derivatives of HA and the different crosslinking methods, as well as the posterior purification processes, carried out in this work may affect the enzymatic degradation. For this reason, the enzymatic degradation on HA-DVS scaffolds and poly(MeHA-co-EA) copolymer networks was compared with that on the different HA-DVS polymer networks (solv_HA:DVS 1:0.9, WI_HA:DVS 1:0.9 and lyo_HA:DVS 1:0.9 samples), with the aim to determine the effect of the conformation and modification of HA chains on the degradation of the different HA-based materials. 
Additionally, the different materials were incubated for 56 days immersed in 0.15 M PBS at $37 \stackrel{\circ}{\circ}$ with the aim to estimate the degradation due to the hydrolysis of water molecules neglecting that due to the effect of hyaluronidases. However, any material showed hydrolytic degradation in the $0.15 \mathrm{M}$ PBS, suggesting that the effect of this medium on the degradation of the different HA-based materials is very low to be noticed. On the other hand, the enzymatic degradation was carried out as accelerating degradation employing a concentration of hyaluronidase of 10 units $/ \mathrm{mL}$, such as previously reported [282]. Surprisingly, the HA-DVS scaffolds with different degree of crosslinking showed no significant degradation up 56 days (Figure 3. 45). Despite the higher concentration in the HA solution employed in the crosslinking of the HA-DVS polymer networks, the solv_HA:DVS 1:0.9 samples were completely degraded after 3 days of enzymatic incubation. The different results found in the degradation of HA-DVS scaffolds and polymer networks corroborate that the posterior processes of purification of the HA-DVS crosslinked materials affect the degradation against hyaluronidase, as well as the mechanical properties and swelling degree, as described above. The idea, above described, that the immersion in acetone of the crosslinked HA with DVS immediately after the crosslinking reaction affects to the hydrogel properties, it is explained because the HA molecules form stable entanglements and possible weak interactions between chains.

On the other hand, the enzymatic degradation of the poly(MeHA-co-EA) copolymer networks yielded a slow degradation of the MeHA component compared with the HA-DVS polymer networks (Figure 3. 46). This phenomenon suggests that the freeze-drying process produces chain entanglement, but the MeHA chains are less contracted than those of the HA-DVS polymer networks summited to phase inversion in acetone after the crosslinking reaction. Moreover, the enzymatic degradation of the MeHA homopolymer network resulted to be complete after 28 days, whereas the degradation was partial in the copolymer networks. This phenomenon may be explained because only the fraction corresponding to the MeHA is susceptible to the hyaluronidase activity. Comparing the weight loss through the enzymatic degradation with the experimental weigh fractions of PEA, and hence MeHA (Table 4. 1), it can be stated that the MeHA component within the copolymer networks was only partially degraded.

In order to determine the possible effect of the purification and drying processes on the stability of HA against the enzymatic degradation, polymer networks based on DVS-crosslinked HA were subjected to different processes after the crosslinking reaction. In addition to the solv_HA:DVS samples, the degradation study was extended to HA crosslinked with DVS in which water was removed using different methods: WI_HA:DVS 1:0.9 samples (described in section 2.2.2) were subjected to phase inversion using acetone, and HA crosslinked with DVS 
in which water is removed by a freeze-drying process (lyo_HA:DVS 1:0.9). These samples were compared with the aim to estimate the effect of the water removal process on chain entanglements, and hence on enzymatic degradation. The results (Figure 4. 4) corroborate that the process employed for removing water has an important effect on the behaviour of crosslinked HA against the hyaluronidase action since the samples subjected to water inversion (WI_HA:DVS 1:0.9) and the lyophilized ones (lyo_HA:DVS 1:0.9) displayed different degradation rates. The WI_HA:DVS 1:0.9 samples were not degraded after 56 days (as in the case of scaffolds), whereas the lyo_HA:DVS 1:0.9 ones were completely degraded between 28 and 56 days (similarly to the MeHA homopolymer networks).

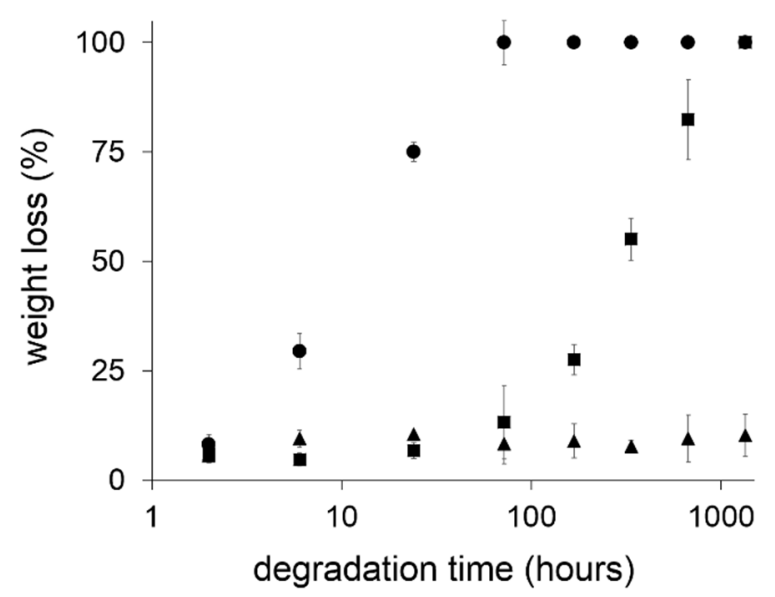

Figure 4. 4. Percentage of weight loss in the different polymer networks based on DVS-crosslinked HA at different degradation times: solv_HA:DVS 1:0.9 (circles), lyo_HA:DVS 1:0.9 (squares) and WI_HA:DVS 1:0.9 (triangles).

The HA-DVS polymer networks purified under different processes were analysed by FTIR with the aim to determine indicia of conformational changes in the HA molecules. Therefore, the different samples displayed similar spectra, but after the deconvolution of the overlapped bands between $1700 \mathrm{~cm}^{-1}$ and $1500 \mathrm{~cm}^{-1}$ (Figure 4. 5) corresponding to the $\mathrm{C}=\mathrm{O}$ stretching of carboxylic acids and amides and the $\mathrm{N}-\mathrm{H}$ bending of amides, some differences in the relative intensity of these bands between each sample were found. The band centred at $1660 \mathrm{~cm}^{-1}$ was predominant in the solv_HA:DVS 1:0.9 samples, while the lyo_HA:DVS 1:0.9 samples, the HA-DVS scaffolds and the WI_HA:DVS 1:0.9 samples showed a predominant band at $1600 \mathrm{~cm}^{-1}$. Therefore, the hypothesis that entanglements 
between HA chains are formed modifying the conformation of the crosslinked HA chains is corroborated.

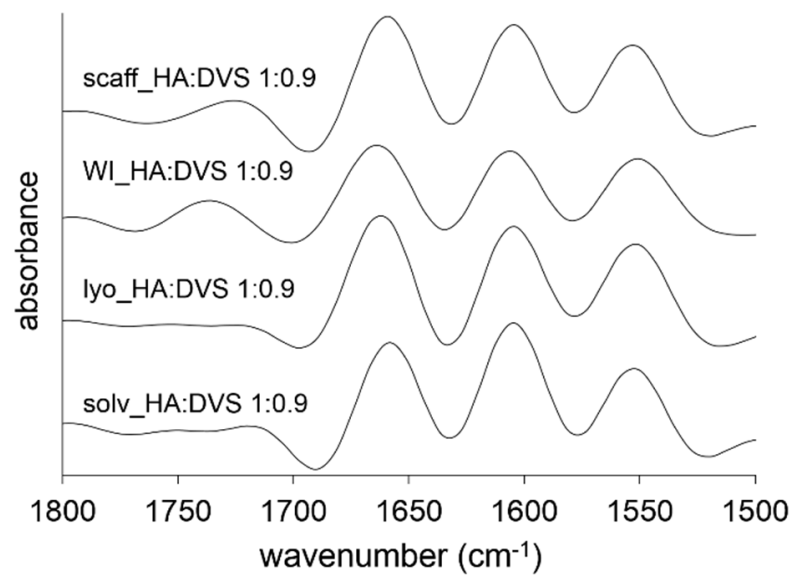

Figure 4. 5. Deconvolution of the bands between 1700 and $1500 \mathrm{~cm}^{-1}$ obtained by FTIR for the HADVS based materials subjected to different purification processes.

In order to determine changes on the HA-based materials due to the activity of the hyaluronidase, the swelling degree was recorded for all the degradation times and microscopic images were taken for each sample. The swelling degree and the internal structure of the different HA-DVS scaffolds resulted in non-significant changes (Figure 3. 47, Figure 3. 49 and Figure 3. 50). However, the HA-DVS polymer networks and the different poly(MeHA-co-EA) copolymer networks change their swelling degree through the enzymatic digestion (Figure 3. 48). The solv_HA:DVS 1:0.9 samples and the MeHA homopolymer networks decrease the swelling degree through the degradation, but at later times such value is noticeably increased. The poly(MeHA-co-EA) copolymer networks showed a slightly reduction of their swelling degree through the enzymatic digestion, due to the reduction of the MeHA content, but this reduction is not in concordance with the real loss of MeHA chains because the pores remaining in the MeHA matrix are filled with media shielding the real swelling degree. On the other hand, the microscopic images of the internal structures in the different copolymer networks were altered after the degradation (Figure 3. 51 and Figure 3. 52). This fact produces certain collapse of the internal structure in the MeHA:PEA 1:0 and MeHA:PEA 1:1 samples, whereas the samples containing the highest PEA content, MeHA:PEA 1:10, resulted in a more open structure, due to the degradation of the MeHA matrix. 
The determination of the molecular weights of the by-products produced during the enzymatic degradation was conducted by chromatography. The study of the supernatants in the different HA-DVS scaffolds resulted in non-presence of byproducts, which is in agreement with the lack of weight loss (Figure 3. 45). This phenomenon suggests that the enzymatic degradation of the HA-DVS scaffolds was not produced at least for 56 days or, in any case, resulted to be low. In the case of the solv_HA:DVS 1:0.9 samples the complete degradation was achieved after 3 days of exposure, showing an earlier degradation after few hours and yielding $\mathrm{HA}$ fragments of relative high weight average molecular weight (between 25 and $50 \mathrm{kDa}$ ) (Figure 3. 53). Moreover, the HA fragments produced after prolonged degradation times (56 days) were further hydrolysed by the hyaluronidase action to lower weight average molecular weights $(4 \mathrm{kDa})$, including an important fraction of oligosaccharides containing between 4 and 8 disaccharide units. The fast degradation observed in the HA-DVS polymer networks corroborates the hypothesis that these materials contain chains without interactions and entanglements, and hence the effective coupling of the enzyme to HA chains to initiate the degradation is accomplished. However, after 56 days of enzymatic degradation the by-products derived from the HA-DVS polymer networks contains important fractions of HA with high molecular weight, suggesting that the crosslinking with DVS, which bond is not disrupted by hyaluronidases, may yield oligosaccharides linked by DVS molecules as final byproducts. Taking into account the Mn $(3.1 \mathrm{KDa})$ of the solv_HA-DVS 1:0.9 fragments after degrading for 56 days (Table 3. 13) and the impossibility of hyaluronidase to cleave the DVS crosslinking, the most fragments are composed of oligosaccharides close to 5 unmodified disaccharides or containing a crosslinking point between themselves. This observation is in agreement with the determination of the degree of crosslinking from swelling experiments since the oligosaccharides between crosslinks were estimated to contain 5.6 disaccharides (Table 3. 5).

The degradation of the different poly(MeHA-co-EA) copolymer networks gave small HA fragments as by-products in the enzymatic degradation, even for short exposure times (Figure 3. 54); this fact may be explained by the relative lower amount of HA chains compared with the HA-DVS polymer networks (these materials were prepared employing solutions with a low HA concentration). However, the degradation in the copolymer networks yielded by-products of high molecular weight for times below 7 days compared with those for longer times, indicating that the enzyme continues cleaving the HA fragments composing MeHA derivative. Besides, the degradation times up 28 days the HA fragments in the copolymer networks were reduced to oligosaccharides containing between 4 and 6 disaccharide units. These results, together with those found on the degradation of the HA-DVS polymer networks, arise that the hyaluronidase employed is limited to cleave HA chains until oligosaccharides containing 4 
disaccharides, as is suggested by other authors [161]. On the other hand, the byproducts derived from the MeHA homopolymer networks resulted in a large amount of the mentioned oligosaccharides, as well as HA fragments with higher molecular weights for all the degradation times. This fact suggests that a fraction of the by-products derived from MeHA homopolymer networks contains an important fraction of crosslinked MeHA fragments, which are not affected by the hyaluronidase activity. In the case of the copolymer networks containing PEA, these bigger fragments were not observed suggesting that the incorporation of EA in the copolymerization reaction leads to MeHA chains crosslinked to the forming PEA network. Therefore, some MeHA fragments remained bonded to the PEA network being not influenced by the enzymatic action of the hyaluronidase. This idea is also suggested comparing the weight loss on the degradation of the copolymer networks (Figure 3. 46) and the calculated PEA weight fraction (Table 4. 1), since the experimental weight fraction of PEA showed higher values than those deducted from the degradation. Additionally, the thermogravimetric analysis of the poly(MeHA-co-EA) copolymer networks under enzymatic digestion for 56 days (Figure 4. 6) displayed that the samples contains some amount of MeHA. Therefore, some MeHA fraction is bonded or occluded in the PEA matrix and is not susceptible of enzymatic degradation.

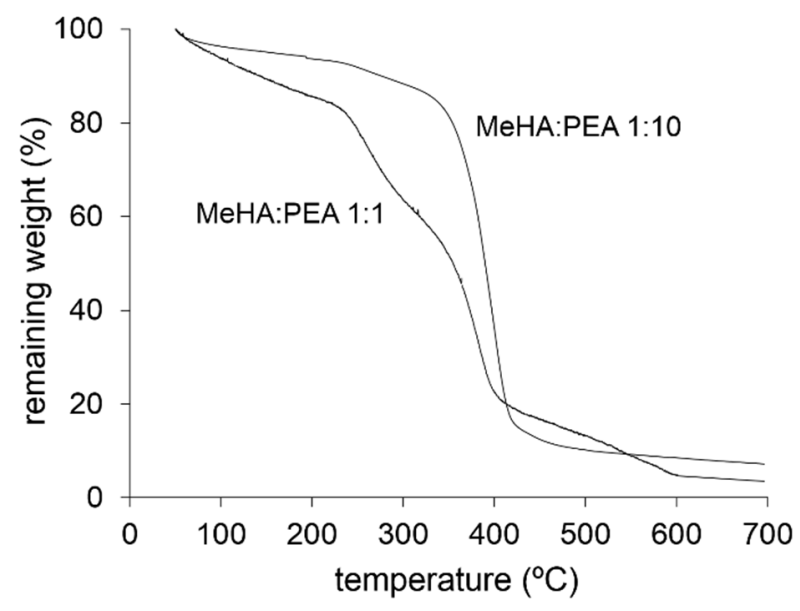

Figure 4. 6. Thermograms of remaining weight of the poly(MeHA-co-EA) copolymer networks after 56 days of enzymatic degradation.

To conclude, the degradation study has demonstrated that the degradation of the HA-based materials could be tailored with the posterior treatments on the crosslinked $\mathrm{HA}$ employed in water removal, as well as by the incorporation of a biostable component such as PEA. In both materials, HA-DVS scaffolds and 
poly(MeHA-co-EA) copolymer networks, the integrity of the structures remain after 56 days of enzymatic degradation. Despite these results cannot be extended to the degradation of a biological environment, the resistance against hyaluronidase activity of the different HA-based materials aimed for their application when longer residence time is required, since the HA molecule and some derivatives thereof are characterized by fast enzymatic degradation rates $[213,372,379]$. On the other hand, taking into account the low methacrylation degree of MeHA (12.5\%), the oligosaccharides derived from the enzymatic degradation are expected to not being composed of modified subunits. Additionally, the controlled delivery of small unmodified oligosaccharides, even at short times, in the different poly(MeHA-co-EA) copolymer networks could improve the regenerative process because small HA fragments promote important biological functions including angiogenesis, cell migration, cell adhesion... [189,190]. 


\subsection{Biological performance}

In the regenerative process of brain tissue, multiple cell types are involved including endothelial, glial and neural cells. After a brain damage, such as stroke or trauma, the capillary network constituting the BBB can be disrupted and the tissues are subjected to an abrupt loss of neural and glial cells, yielding a loss of the biological functions usually permanent [3]. The BBB is a unique structure and shows several differences with respect to the capillary network of other tissues, which result in an impermeable structure for molecules and peripheral cells. Additionally, these unique characteristics of the BBB are related with endothelial cells, as well as their interactions with brain cells including astrocytes, pericytes and neurons. Therefore, in the present work, a preliminary study was carried out using different cells implied in the BBB maintenance (astrocytes, neurons and endothelial cells), with the aim to address their interaction and viability in the HADVS scaffolds. However, the different cell types employed here are models of the original ones: U373 cell line as astrocyte model, SH-SY5Y cell line as neuronal cell model, and primary endothelial cells from pig brains and hCMEC/D3 cell line as endothelial cell models.

First of all, the cell viability in contact with material extracts (HA-DVS scaffolds and poly(MeHA-co-EA) copolymer networks) was addressed determining the cytotoxicity (Figure 3. 55 and Figure 3. 56), induction of cell apoptosis (Figure 3. 57), effect on proliferation (Figure 3. 58) and the inflammatory effect (Figure 3. 59) and taking as reference adequate controls. From the study of viability arises that the extracts of both materials, HA-DVS scaffolds and poly(MeHA-co-EA) copolymer networks, do not induce adverse effects, in terms of cytotoxicity and inflammation, over the different cell lines employed in the cell cultures.

The initial cell colonization of the different cell types (glial, neural and endothelial cells) was enhanced in the HA-DVS scaffolds with higher amounts of DVS. This fact may be related with the change in the scaffolds properties induced by the degree of crosslinking. Several authors have suggested that softer HA based hydrogels enhance the adhesion of neurons but not that of glial cells [380]. Taking into account the enhancement of cell colonization by all cell populations and the similar properties of the scaff_HA:DVS 1:1.5 samples, it indicates that this material is a good candidate for further brain implants. Scaffolds from HA derivatives resulted in a low cell attachment because of the anionic nature of HA. However, the presence of specific proteins and other factors could enhance the ingrowth of cells in the scaffolds such as is corroborated in some studies [286].

Despite the low density, the astrocyte-like cell line cultured in the scaffolds shows their characteristic elongated shape (Figure 3. 60) indicating the absence of hypertrophic astrocytes, which are associated with the glial scar. On the other hand, the absence of stimulus for neurite development explains that the neural- 
like cells did not display longer processes, whereas some cells displayed longer neurites when they were cultured in both controls and scaffolds inducing certain degree of neurite development by themselves or due to the presence of fibronectin (Figure 3. 61). The endothelial-like cells have demonstrated to be morphologically homogeneous forming confluent monolayers when they are cultured on well plates, moreover this structure is observed when they are seeded on the scaffolds showing monolayers surrounding the pores of the scaffolds (Figure 3. 62). Additionally, the endothelial-like cells resulted to yield denser populations and the structures became more evident when they were cultured together with neural- and/or glial-like cells (Figure 3. 64 and Figure 3. 65). This phenomenon may be induced by U373 and SH-SY5Y cells, since these cells have demonstrated to deliver neurotrophic factors, such as VEGF, favouring the maintenance of the endothelial cell phenotype [381,382].

The colonization of the scaffolds by the different endothelial cells suggests that the scaff_HA:DVS 1:1.5 samples may act as a good substrate for brain regeneration purposes because the ingrowth of endothelial cells could guarantee the nutrient transport required in the regenerative process. Moreover, the colonization of the scaffolds by the endothelial cells is viable at least for 14 days (Figure 3. 66).

On the other hand, preliminary studies of cell cultured on FN- coated poly(MeHAco-EA) copolymer and PEA homopolymer networks were performed with the aim of determining the effect of the PEA component. In the following figure is displayed the nuclei staining of the glial-, neural-, and endothelial-like cells in the pure MeHA and PEA homopolymer networks and in the poly(MeHA-co-EA) copolymer networks. 


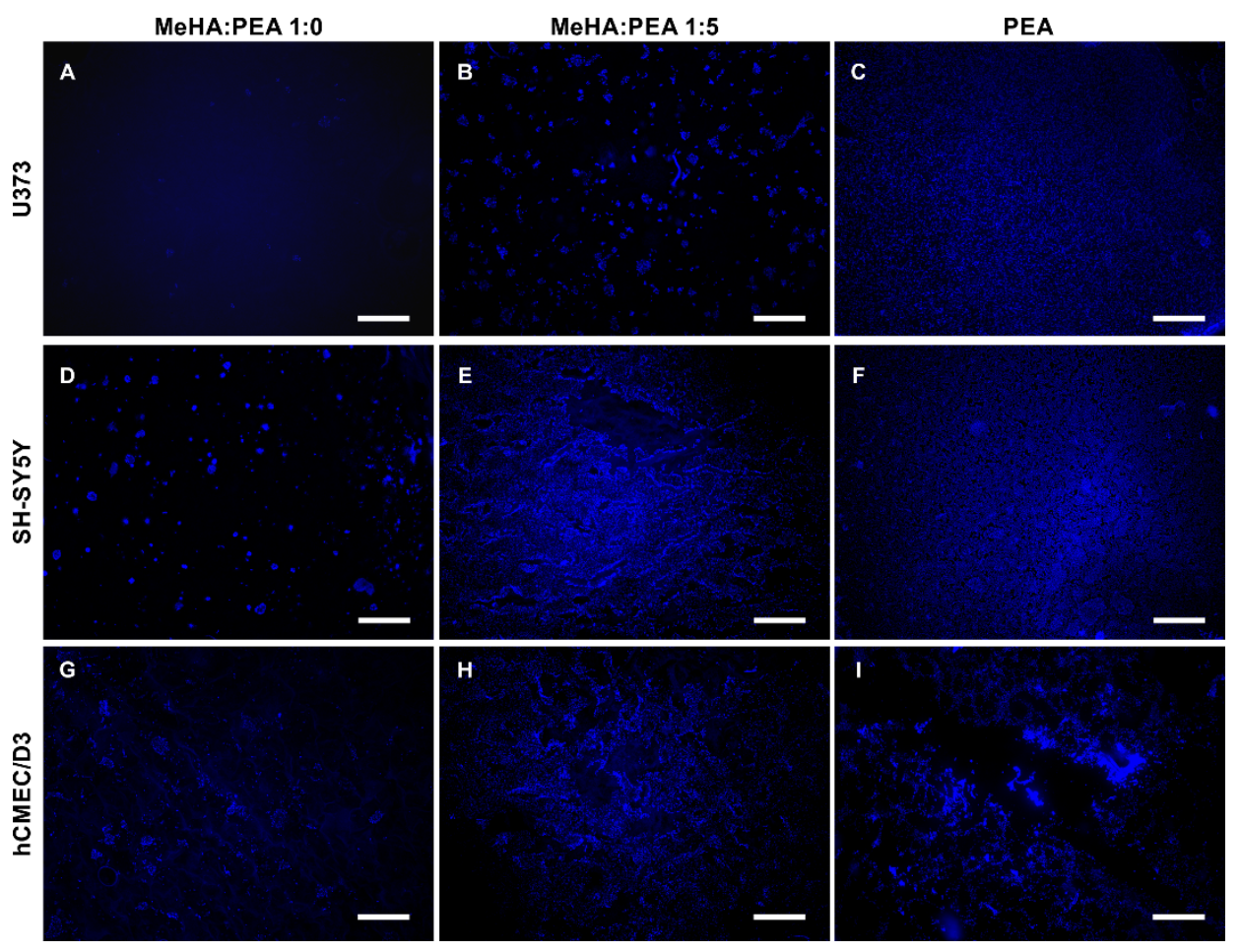

Figure 4. 7. Hoechst staining of U373, SH-SY5Y and hCMEC/D3 cell lines seeded on different poly(MeHA-co-EA) copolymer and PEA homopolymer networks and incubated for 7 days. Scale bar $500 \mu \mathrm{m}$.

Figure 4.7 shows, as expected, that the higher the PEA content in the copolymer networks, the higher the density of the different cell populations. These results are in agreement with previous works, in which it has been demonstrated that PEA is a good substrate for different cells, including endothelial [354], glial [353] and neural [21] ones. Additionally, the different cells seeded on the MeHA homopolymer network display a low cell density compared with the materials containing PEA and they are arranged as isolated clusters, which are typical of HA based hydrogels [73]. On the other hand, cell density is increased in the copolymer networks, even the SH-SY5Y and hCMEC/D3 cell populations are arranged forming a continuous monolayer. Despite this study was considered as a preliminary one, the results found here aimed us to consider that the copolymer networks are a good substrate for the different cells involved in brain regeneration. This hypothesis is also corroborated by immunostaining studies of the different cells seeded as monocultures on PEA surface coated with FN and showed in the following figure. 


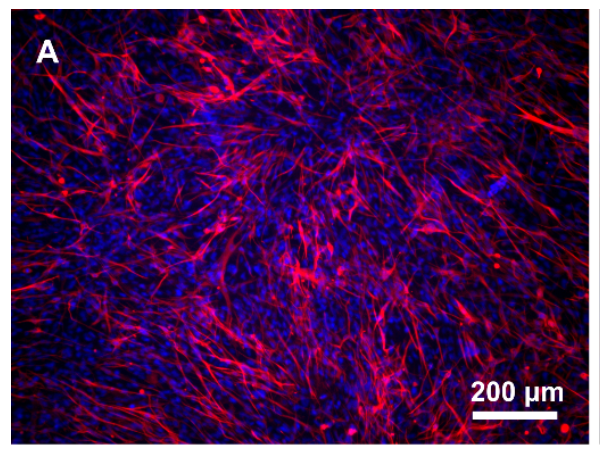

\section{B}

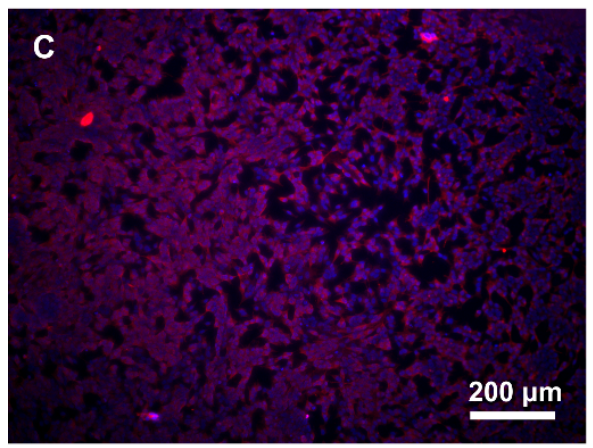

D

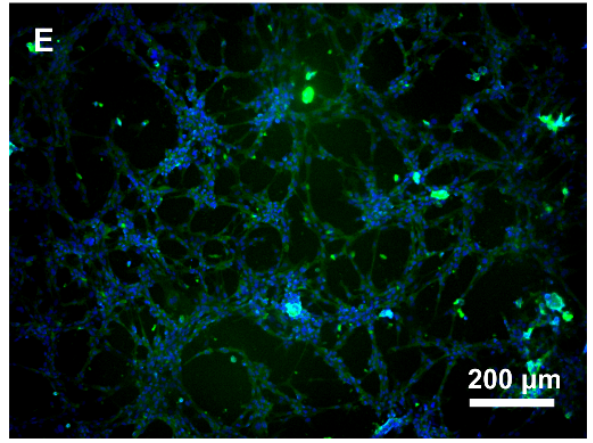

$\mathbf{F}$

$100 \mu \mathrm{m}$

G

H

$100 \mu \mathrm{m}$

Figure 4. 8. Immunostaining studies of different cells seeded as monocultures on the PEA homopolymer networks and incubated for 7 days. Expression of GFAP (red) in U373 cell line (A-B), Tuj-1 (red) in SH-SY5Y cell line (C-D) and CD-31 (green) in hCMEC/D3 cell line (E-F) and pBEC primary cells $(\mathrm{G}-\mathrm{H})$. Cell nuclei were stained in blue with Hoechst. 
The immunostaining studies of the different cells seeded as monocultures on PEA (Figure 4. 8) corroborate the good biocompatibility of this material with the astrocyte-, neural- and endothelial-like cells. The different cell populations cover the material surface arranging in continuous structures. The U373 cells shows a high density covering the complete surface and, in the most cases, these cells display an elongated shape. Similarly, the SH-SY5Y cells cover the complete surface of PEA, and they are arranged in continuous clusters showing contact between cells. Moreover, some SH-SY5Y cells display neurite growth when localized in regions containing low cell density. In general, these processes are short ones, but Figure 4. 8-D suggests that the SH-SY5Y cells seeded on the PEA substrates express neurites, which also may form connections between cells. On the other hand, the cultures of hCMEC/D3 and pBEC cell populations on the PEA surface resulted in cells forming different structures depending on the cell type. In the case of hCMEC/D3 cell line, cells were arranged forming networklike structures. This phenomenon, could be produced due to the early fibrillogenesis of FN onto PEA surfaces observed by other authors [383]. Additionally, the hCMEC/D3 are forming tubular-like structures and connections between themselves, but this phenomenon should be corroborated with additional studies. In the case of pBECs cultures on PEA, cell morphology resulted to be similar to that observed for pBECs seeded on controls (Figure 3 . 63). Therefore, pBECs are arranged forming continuous monolayers with a close contact between themselves.

In the present work, the different biological studies showed no cytotoxic and inflammatory effects on cells exposed to the extract of the different materials. Besides, the results suggest the possibility to improve the initial cell colonization increasing the degree of crosslinking in the HA-DVS scaffolds and increasing the PEA content in the poly(MeHA-co-EA) copolymer networks. However, the BBB structure is a very complex system, and hence further studies are required with the aim to assess the effect of each cell type on the signal transduction of each other (crosstalk) and determine the unique characteristics of the BBB (low permeability, tight junctions between endothelial cells, specific carriers...). 

CHAPTER V

CONCLUSIONS 



\section{Conclusions}

Polymer networks based on HA have been developed by different methods: divinyl sulfone crosslinked $\mathrm{HA}$ and copolymers of methacrylated HA with a biostable component, PEA. The developed biomaterials permit to produce 3D structures (scaffolds) containing interconnected pores with sizes that allow access for cell colonization. Therefore, these structures may be employed in the regenerative processes of soft tissues, such as brain tissue. Varying the composition of the biomaterials has permitted to tune the mechanical and physical properties of the different polymer networks. The different cells types, modelling those involved in brain regeneration (neurons, glia and endothelial cells), have resulted to be viable for the different materials. The materials developed (HA-DVS scaffolds and poly(MeHA-co-EA) copolymer networks) have demonstrated resistance against the hyaluronidase activity.

The characterization of each system has proportioned particular conclusions.

Divinyl sulfone- crosslinked hyaluronan

- Comparing the degree of crosslinking of HA with divinyl sulfone determined by semi-empirical methods with the real content of DVS estimated by experimental methods, it is deduced that the crosslinking reaction yielded low efficacies.

- It was possible to develop a repetitive process to fabricate scaffolds based on HA crosslinked with DVS.

- The mechanical and physical properties of the HA-DVS scaffolds were tailored by varying the molar ratio of HA to DVS. Additionally, the scaffolds showed similar mechanical properties with respect to those of the brain tissue.

- The methods employed in the purification of the crosslinked polymer networks cause conformational changes in the HA chains, which influence on their resistance against the hyaluronidase activity.

- The degree of crosslinking, and hence the mechanical properties, of the crosslinked HA scaffolds have resulted to influence the initial cell colonization of glial, neural and endothelial cells. 
Poly(MeHA-co-EA) copolymer networks

- Varying the composition of the copolymer networks is interesting for obtaining biomaterials with tuneable mechanical and physical properties.

- The experimental weight fraction of PEA in the copolymer networks, determined by different methods, resulted to be lower than the nominal weight fraction of PEA (EA weight fraction used in the copolymerization reaction).

- The incorporation of a biostable matrix, the PEA component, in the copolymer networks has permitted to develop semi-biodegradable materials. Moreover, the enzymatic digestion on the copolymer networks yielded to a sustained release of HA fragments with angiogenic properties.

- The poly(MeHA-co-EA) copolymer networks might be employed in the development of interconnected porous scaffolds controlling pore sizes.

- The copolymer networks have shown no cytotoxic effects on neural, glial and endothelial cells. Furthermore, these biomaterials have no significant inflammatory effect on the endothelial cells.

- The preliminary biological studies suggest that the incorporation of PEA might help the compatibility of the copolymer networks with glial, neural and endothelial cells. 




\section{References}

[1] Dekkers MPJ, Nikoletopoulou V, Barde Y-A. Cell biology in neuroscience: Death of developing neurons: new insights and implications for connectivity. The Journal of Cell Biology 2013; 203(3) 385-93.

[2] Yiu G, He Z. Glial inhibition of CNS axon regeneration. Nature Reviews Neuroscience 2006; 7(8) 617-27.

[3] Horner PJ, Gage FH. Regenerating the damaged central nervous system. Nature 2000; 407(6807) 963-70.

[4] Galvao R, Garcia-Verdugo JM, Alvarez-Buylla A. Adult neural stem cells; prospects for brain reapair. In: García-Olmo D, García-Verdugo JM, Alemany A, Gutiérrez-Fuentes JA, editors. Cell therapy, Madrid (SP): McGraw-HillInteramericana; 2008.

[5] Willerth SM, Sakiyama-Elbert SE. Cell therapy for spinal cord regeneration. Advanced Drug Delivery Reviews 2008; 60(2) 263-76.

[6] Saberi H, Firouzi M, Habibi Z, Moshayedi P, Aghayan HR, Arjmand B, et al. Safety of intramedullary Schwann cell transplantation for postrehabilitation spinal cord injuries: 2-year follow-up of 33 cases. Journal of Neurosurgery Spine 2011; 15(5) 515-25.

[7] Zhou X-H, Ning G-Z, Feng S-Q, Kong X-H, Chen J-T, Zheng Y-F, et al. Transplantation of autologous activated Schwann cells in the treatment of spinal cord injury: six cases, more than five years of follow-up. Cell Transplantation 2012; 21(Suppl 1) S39-47.

[8] Lakatos A, Barnett SC, Franklin RJM. Olfactory ensheathing cells induce less host astrocyte response and chondroitin sulphate proteoglycan expression than Schwann cells following transplantation into adult CNS white matter. Experimental Neurology 2003; 184(1) 237-46.

[9] Popovich PG, Guan Z, Wei P, Huitinga I, van Rooijen N, Stokes BT. Depletion of hematogenous macrophages promotes partial hindlimb recovery and neuroanatomical repair after experimental spinal cord injury. Experimental Neurology 1999; 158(2) 351-65.

[10] Miron VE, Franklin RJM. Macrophages and CNS remyelination. Journal of Neurochemistry 2014;

[11] Tetzlaff W, Okon EB, Karimi-Abdolrezaee S, Hill CE, Sparling JS, Plemel JR, et al. A systematic review of cellular transplantation therapies for spinal cord injury. Journal of Neurotrauma 2011; 28(8) 1611-82.

[12] Techangamsuwan S, Imbschweiler I, Kreutzer R, Kreutzer M, Baumgärtner W, Wewetzer K. Similar behaviour and primate-like properties of adult canine Schwann cells and olfactory ensheathing cells in long-term culture. Brain Research 2008; 1240 31-8.

[13] Imaizumi T, Lankford KL, Waxman SG, Greer CA, Kocsis JD. Transplanted olfactory ensheathing cells remyelinate and enhance axonal conduction in the demyelinated dorsal columns of the rat spinal cord. The Journal of Neuroscience : The Official Journal of the Society for Neuroscience 1998; 18(16) 6176-85.

[14] Yan H, Nie X, Kocsis JD. Hepatocyte growth factor is a mitogen for olfactory ensheathing cells. Journal of Neuroscience Research 2001; 66(4) 698-704.

[15] Granger N, Blamires H, Franklin RJM, Jeffery ND. Autologous olfactory mucosal cell transplants in clinical spinal cord injury: a randomized double-blinded trial in a canine translational model. Brain : A Journal of Neurology 2012; 135(Pt 11) 322737.

[16] Stamegna JC, Felix M., Roux-Peyronnet J, Rossi V, Féron F, Gauthier P, et al. Nasal OEC transplantation promotes respiratory recovery in a subchronic rat 
model of cervical spinal cord contusion. Experimental Neurology 2011; 229(1) 120-31.

[17] Shi X, Kang Y, Hu Q, Chen C, Yang L, Wang K, et al. A long-term observation of olfactory ensheathing cells transplantation to repair white matter and functional recovery in a focal ischemia model in rat. Brain Research 2010; 1317 257-67.

[18] Su Z, He C. Olfactory ensheathing cells: biology in neural development and regeneration. Progress in Neurobiology 2010; 92(4) 517-32.

[19] Honmou O, Onodera R, Sasaki M, Waxman SG, Kocsis JD. Mesenchymal stem cells: therapeutic outlook for stroke. Trends in Molecular Medicine 2012; 18(5) 292-7.

[20] Setoguchi T, Nakashima K, Takizawa T, Yanagisawa M, Ochiai W, Okabe M, et al. Treatment of spinal cord injury by transplantation of fetal neural precursor cells engineered to express BMP inhibitor. Experimental Neurology 2004; 189(1) 3344.

[21] Martínez-Ramos C, Lainez S, Sancho F, García Esparza MA, Planells-Cases R, García Verdugo JM, et al. Differentiation of postnatal neural stem cells into glia and functional neurons on laminin-coated polymeric substrates. Tissue Engineering Part A 2008; 14(8) 1365-75.

[22] Cepeda IL, Flores J, Cornfeldt ML, O'Kusky JR, Doudet DJ. Human retinal pigment epithelial cell implants ameliorate motor deficits in two rat models of Parkinson disease. Journal of Neuropathology and Experimental Neurology 2007; 66(7) 576-84.

[23] Doudet DJ, Cornfeldt ML, Honey CR, Schweikert AW, Allen RC. PET imaging of implanted human retinal pigment epithelial cells in the MPTP-induced primate model of Parkinson's disease. Experimental Neurology 2004; 189(2) 361-8.

[24] Nógrádi A, Szabó A. Transplantation of embryonic neurones to replace missing spinal motoneurones. Restorative Neurology and Neuroscience 2008; 26(2-3) 215-23.

[25] Okano H. Strategies toward CNS-regeneration using induced pluripotent stem cells. Genome Informatics International Conference on Genome Informatics 2009; 23(1) 217-20.

[26] Pérez-Garnes M, Barcia Albacar JA, Gómez-Pinedo U, Monleón-Pradas M, Vallés-Lluch A. Materials for central nervous system tissue engineering. Regenerative Medicine and tissue engineering, InTech; ISBN 978-953-51-41143; 2014.

[27] Clements IP, Mukhatyar VJ, Srinivasan A, Bentley JT, Andreasen DS, Bellamkonda R V. Regenerative scaffold electrodes for peripheral nerve interfacing. IEEE Transactions on Neural Systems and Rehabilitation Engineering: A Publication of the IEEE Engineering in Medicine and Biology Society 2013; 21(4) 554-66.

[28] Huang W-C, Yao C-K, Liao J-D, Lin C-CK, Ju M-S. Enhanced Schwann cell adhesion and elongation on a topographically and chemically modified poly(Llactic acid) film surface. Journal of Biomedical Materials Research Part A 2011; 99(2) 158-65.

[29] Gertz CC, Leach MK, Birrell LK, Martin DC, Feldman EL, Corey JM. Accelerated neuritogenesis and maturation of primary spinal motor neurons in response to nanofibers. Developmental Neurobiology 2010; 70(8) 589-603.

[30] Bellamkonda $\mathrm{R}$ V. Peripheral nerve regeneration: an opinion on channels, scaffolds and anisotropy. Biomaterials 2006; 27(19) 3515-8.

[31] Yang IH, Co CC, Ho C-C. Controlling neurite outgrowth with patterned substrates. Journal of Biomedical Materials Research Part A 2011; 97(4) 451-6.

[32] Béduer A, Vieu C, Arnauduc F, Sol J-C, Loubinoux I, Vaysse L. Engineering of adult human neural stem cells differentiation through surface micropatterning. Biomaterials 2012; 33(2) 504-14. 
[33] Yao L, Wang S, Cui W, Sherlock R, O'Connell C, Damodaran G, et al. Effect of functionalized micropatterned PLGA on guided neurite growth. Acta Biomaterialia 2009; 5(2) 580-8.

[34] Goldner JS, Bruder JM, Li G, Gazzola D, Hoffman-Kim D. Neurite bridging across micropatterned grooves. Biomaterials 2006; 27(3) 460-72.

[35] Krsko P, McCann TE, Thach T-T, Laabs TL, Geller HM, Libera MR. Length-scale mediated adhesion and directed growth of neural cells by surface-patterned poly(ethylene glycol) hydrogels. Biomaterials 2009; 30(5) 721-9.

[36] Johansson F, Carlberg P, Danielsen N, Montelius L, Kanje M. Axonal outgrowth on nano-imprinted patterns. Biomaterials 2006; 27(8) 1251-8.

[37] Mahoney MJ, Chen RR, Tan J, Saltzman WM. The influence of microchannels on neurite growth and architecture. Biomaterials 2005; 26(7) 771-8.

[38] Seidlits SK, Lee JY, Schmidt CE. Nanostructured scaffolds for neural applications. Nanomedicine 2008; 3(2) 183-99.

[39] Lim SH, Liu XY, Song H, Yarema KJ, Mao H-Q. The effect of nanofiber-guided cell alignment on the preferential differentiation of neural stem cells. Biomaterials 2010; 31(34) 9031-9.

[40] Xie J, Willerth SM, Li X, Macewan MR, Rader A, Sakiyama-Elbert SE, et al. The differentiation of embryonic stem cells seeded on electrospun nanofibers into neural lineages. Biomaterials 2009; 30(3) 354-62.

[41] Schnell E, Klinkhammer K, Balzer S, Brook G, Klee D, Dalton P, et al. Guidance of glial cell migration and axonal growth on electrospun nanofibers of poly-epsiloncaprolactone and a collagen/poly-epsilon-caprolactone blend. Biomaterials 2007; 28(19) 3012-25.

[42] Mukhatyar VJ, Salmerón-Sánchez M, Rudra S, Mukhopadaya S, Barker TH, García AJ, et al. Role of fibronectin in topographical guidance of neurite extension on electrospun fibers. Biomaterials 2011; 32(16) 3958-68.

[43] Corey JM, Lin DY, Mycek KB, Chen Q, Samuel S, Feldman EL, et al. Aligned electrospun nanofibers specify the direction of dorsal root ganglia neurite growth. Journal of Biomedical Materials Research Part A 2007; 83(3) 636-45.

[44] Wen X, Tresco PA. Effect of filament diameter and extracellular matrix molecule precoating on neurite outgrowth and Schwann cell behavior on multifilament entubulation bridging device in vitro. Journal of Biomedical Materials Research Part A 2006; 76(3) 626-37.

[45] Pérez-Garnes M, Martínez-Ramos C, Barcia JA, Escobar Ivirico JL, GómezPinedo U, Vallés-Lluch $A$, et al. One-dimensional migration of olfactory ensheathing cells on synthetic materials: experimental and numerical characterization. Cell Biochemistry and Biophysics 2013; 65(1) 21-36.

[46] Vitale-Brovarone C, Novajra G, Lousteau J, Milanese D, Raimondo S, Fornaro M. Phosphate glass fibres and their role in neuronal polarization and axonal growth direction. Acta Biomaterialia 2012; 8(3) 1125-36.

[47] Yu W, Jiang X, Cai M, Zhao W, Ye D, Zhou Y, et al. A novel electrospun nerve conduit enhanced by carbon nanotubes for peripheral nerve regeneration. Nanotechnology 2014; 25(16) 165102.

[48] Clements IP, Kim Y, English AW, Lu X, Chung A, Bellamkonda R V. Thin-film enhanced nerve guidance channels for peripheral nerve repair. Biomaterials 2009; 30(23-24) 3834-46.

[49] Martínez-Ramos C, Vallés-Lluch A, Verdugo JMG, Ribelles JLG, Barcia Albacar JA, Orts $A B$, et al. Channeled scaffolds implanted in adult rat brain. Journal of Biomedical Materials Research Part A 2012; 100(12) 3276-86.

[50] Yu TT, Shoichet MS. Guided cell adhesion and outgrowth in peptide-modified channels for neural tissue engineering. Biomaterials 2005; 26(13) 1507-14. 
[51] Sanghvi AB, Miller KP-H, Belcher AM, Schmidt CE. Biomaterials functionalization using a novel peptide that selectively binds to a conducting polymer. Nature Materials 2005; 4(6) 496-502.

[52] Voge CM, Stegemann JP. Carbon nanotubes in neural interfacing applications. Journal of Neural Engineering 2011; 8(1) 011001.

[53] Young T-H, Lin U-H, Lin D-J, Chang H-H, Cheng L-P. Immobilization of L-lysine on microporous PVDF membranes for neuron culture. Journal of Biomaterials Science Polymer Edition 2009; 20(5-6) 703-20.

[54] Schmidt CE, Leach JB. Neural tissue engineering: strategies for repair and regeneration. Annual Review of Biomedical Engineering 2003; 5 293-347.

[55] Wong JY, Langer R, Ingber DE. Electrically conducting polymers can noninvasively control the shape and growth of mammalian cells. Proceedings of the National Academy of Sciences of the United States of America 1994; 91(8) 3201-4.

[56] Song H-K, Toste B, Ahmann K, Hoffman-Kim D, Palmore GTR. Micropatterns of positive guidance cues anchored to polypyrrole doped with polyglutamic acid: a new platform for characterizing neurite extension in complex environments. Biomaterials 2006; 27(3) 473-84.

[57] Mattioli-Belmonte M, Giavaresi G, Biagini G, Virgili L, Giacomini M, Fini M, et al. Tailoring biomaterial compatibility: in vivo tissue response versus in vitro cell behavior. The International Journal of Artificial Organs 2003; 26(12) 1077-85.

[58] Richardson-Burns SM, Hendricks JL, Martin DC. Electrochemical polymerization of conducting polymers in living neural tissue. Journal of Neural Engineering 2007; 4(2) L6-L13.

[59] Wang X, Gu X, Yuan C, Chen S, Zhang P, Zhang T, et al. Evaluation of biocompatibility of polypyrrole in vitro and in vivo. Journal of Biomedical Materials Research Part A 2004; 68(3) 411-22.

[60] Asplund M, Thaning E, Lundberg J, Sandberg-Nordqvist AC, Kostyszyn B, Inganäs $\mathrm{O}$, et al. Toxicity evaluation of PEDOT/biomolecular composites intended for neural communication electrodes. Biomedical Materials 2009; 4(4) 045009.

[61] Guimard NK, Gomez N, Schmidt CE. Conducting polymers in biomedical engineering. Progress in Polymer Science 2007; 32(8-9) 876-921.

[62] Sisken BF, Kanje M, Lundborg G, Herbst E, Kurtz W. Stimulation of rat sciatic nerve regeneration with pulsed electromagnetic fields. Brain Research 1989; 485(2) 309-16.

[63] Zhao M, Forrester J V, McCaig CD. A small, physiological electric field orients cell division. Proceedings of the National Academy of Sciences of the United States of America 1999; 96(9) 4942-6.

[64] Yamada M, Tanemura K, Okada S, Iwanami A, Nakamura M, Mizuno H, et al. Electrical stimulation modulates fate determination of differentiating embryonic stem cells. Stem Cells 2007; 25(3) 562-70.

[65] Wood M, Willits RK. Short-duration, DC electrical stimulation increases chick embryo DRG neurite outgrowth. Bioelectromagnetics 2006; 27(4) 328-31.

[66] Alexander JK, Fuss B, Colello RJ. Electric field-induced astrocyte alignment directs neurite outgrowth. Neuron Glia Biology 2006; 2(2) 93-103.

[67] Ghasemi-Mobarakeh L, Prabhakaran MP, Morshed M, Nasr-Esfahani MH, Ramakrishna S. Electrical stimulation of nerve cells using conductive nanofibrous scaffolds for nerve tissue engineering. Tissue Engineering Part A 2009; 15(11) 3605-19.

[68] Thompson BC, Richardson RT, Moulton SE, Evans AJ, O'Leary S, Clark GM, et al. Conducting polymers, dual neurotrophins and pulsed electrical stimulation-dramatic effects on neurite outgrowth. Journal of Controlled Release 2010; 141(2) 161-7. 
[69] Edalatmanesh M-A, Matin MM, Neshati Z, Bahrami A-R, Kheirabadi M. Systemic transplantation of mesenchymal stem cells can reduce cognitive and motor deficits in rats with unilateral lesions of the neostriatum. Neurological Research 2010; 32(2) 166-72.

[70] Xie J, Macewan MR, Willerth SM, Li X, Moran DW, Sakiyama-Elbert SE, et al. Conductive Core-Sheath Nanofibers and Their Potential Application in Neural Tissue Engineering. Advanced Functional Materials 2009; 19(14) 2312-8.

[71] Patel N, Poo MM. Orientation of neurite growth by extracellular electric fields. The Journal of Neuroscience: The Official Journal of the Society for Neuroscience 1982; 2(4) 483-96.

[72] Elkin BS, Azeloglu EU, Costa KD, Morrison B. Mechanical heterogeneity of the rat hippocampus measured by atomic force microscope indentation. Journal of Neurotrauma 2007; 24(5) 812-22.

[73] Seidlits SK, Khaing ZZ, Petersen RR, Nickels JD, Vanscoy JE, Shear JB, et al. The effects of hyaluronic acid hydrogels with tunable mechanical properties on neural progenitor cell differentiation. Biomaterials 2010; 31(14) 3930-40.

[74] Georges PC, Miller WJ, Meaney DF, Sawyer ES, Janmey PA. Matrices with compliance comparable to that of brain tissue select neuronal over glial growth in mixed cortical cultures. Biophysical Journal 2006; 90(8) 3012-8.

[75] Banerjee A, Arha M, Choudhary S, Ashton RS, Bhatia SR, Schaffer D V, et al. The influence of hydrogel modulus on the proliferation and differentiation of encapsulated neural stem cells. Biomaterials 2009; 30(27) 4695-9.

[76] Man AJ, Davis HE, Itoh A, Leach JK, Bannerman P. Neurite outgrowth in fibrin gels is regulated by substrate stiffness. Tissue Engineering Part A 2011; 17(2324) 2931-42.

[77] Gunn JW, Turner SD, Mann BK. Adhesive and mechanical properties of hydrogels influence neurite extension. Journal of Biomedical Materials Research Part A 2005; 72(1) 91-7.

[78] Leach JB, Brown XQ, Jacot JG, Dimilla PA, Wong JY. Neurite outgrowth and branching of PC12 cells on very soft substrates sharply decreases below a threshold of substrate rigidity. Journal of Neural Engineering 2007; 4(2) 26-34.

[79] Stabenfeldt SE, LaPlaca MC. Variations in rigidity and ligand density influence neuronal response in methylcellulose-laminin hydrogels. Acta Biomaterialia 2011; 7(12) 4102-8.

[80] Cheng C-M, LeDuc PR, Lin Y-W. Localized bimodal response of neurite extensions and structural proteins in dorsal-root ganglion neurons with controlled polydimethylsiloxane substrate stiffness. Journal of Biomechanics 2011 ; 44(5) 856-62.

[81] Zelzer M, Alexander MR, Russell NA. Hippocampal cell response to substrates with surface chemistry gradients. Acta Biomaterialia 2011; 7(12) 4120-30.

[82] Lee SJ, Khang G, Lee YM, Lee HB. The effect of surface wettability on induction and growth of neurites from the PC-12 cell on a polymer surface. Journal of Colloid and Interface Science 2003; 259(2) 228-35.

[83] Lamour G, Eftekhari-Bafrooei A, Borguet E, Souès S, Hamraoui A. Neuronal adhesion and differentiation driven by nanoscale surface free-energy gradients. Biomaterials 2010; 31(14) 3762-71.

[84] Tate CC, Shear DA, Tate MC, Archer DR, Stein DG, LaPlaca MC. Laminin and fibronectin scaffolds enhance neural stem cell transplantation into the injured brain. Journal of Tissue Engineering and Regenerative Medicine 2009; 3(3) 20817.

[85] Wei YT, Tian WM, Yu X, Cui FZ, Hou SP, Xu QY, et al. Hyaluronic acid hydrogels with IKVAV peptides for tissue repair and axonal regeneration in an injured rat brain. Biomedical Materials 2007; 2(3) S142-6. 
[86] Nisbet DR, Rodda AE, Horne MK, Forsythe JS, Finkelstein DI. Neurite infiltration and cellular response to electrospun polycaprolactone scaffolds implanted into the brain. Biomaterials 2009; 30(27) 4573-80.

[87] Wong DY, Krebsbach PH, Hollister SJ. Brain cortex regeneration affected by scaffold architectures. Journal of Neurosurgery 2008; 109(4) 715-22.

[88] Wang Y, Cooke MJ, Morshead CM, Shoichet MS. Hydrogel delivery of erythropoietin to the brain for endogenous stem cell stimulation after stroke injury. Biomaterials 2012; 33(9) 2681-92.

[89] Guo J, Leung KKG, Su H, Yuan Q, Wang L, Chu T-H, et al. Self-assembling peptide nanofiber scaffold promotes the reconstruction of acutely injured brain. Nanomedicine: Nanotechnology, Biology, and Medicine 2009; 5(3) 345-51.

[90] Xiong Y, Qu C, Mahmood A, Liu Z, Ning R, Li Y, et al. Delayed transplantation of human marrow stromal cell-seeded scaffolds increases transcallosal neural fiber length, angiogenesis, and hippocampal neuronal survival and improves functional outcome after traumatic brain injury in rats. Brain Research 2009; 1263 183-91.

[91] Qu C, Xiong Y, Mahmood A, Kaplan DL, Goussev A, Ning R, et al. Treatment of traumatic brain injury in mice with bone marrow stromal cell-impregnated collagen scaffolds. Journal of Neurosurgery 2009; 111(4) 658-65.

[92] Tate MC, Shear DA, Hoffman SW, Stein DG, LaPlaca MC. Biocompatibility of methylcellulose-based constructs designed for intracerebral gelation following experimental traumatic brain injury. Biomaterials 2001; 22(10) 1113-23.

[93] Lu D, Mahmood A, Qu C, Hong X, Kaplan D, Chopp M. Collagen scaffolds populated with human marrow stromal cells reduce lesion volume and improve functional outcome after traumatic brain injury. Neurosurgery 2007; 61(3) 596603.

[94] Mingyu C, Kai G, Jiamou L, Yandao G, Nanming Z, Xiufang Z. Surface modification and characterization of chitosan film blended with poly-L-lysine. Journal of Biomaterials Applications 2004; 19(1) 59-75.

[95] Lewitus DY, Smith KL, Shain W, Bolikal D, Kohn J. The fate of ultrafast degrading polymeric implants in the brain. Biomaterials 2011; 32(24) 5543-50.

[96] Azemi E, Lagenaur CF, Cui XT. The surface immobilization of the neural adhesion molecule L1 on neural probes and its effect on neuronal density and gliosis at the probe/tissue interface. Biomaterials 2011; 32(3) 681-92.

[97] Moon SU, Kim J, Bokara KK, Kim JY, Khang D, Webster TJ, et al. Carbon nanotubes impregnated with subventricular zone neural progenitor cells promotes recovery from stroke. International Journal of Nanomedicine 2012; 7 2751-65.

[98] Wong DY, Hollister SJ, Krebsbach PH, Nosrat C. Poly(epsilon-caprolactone) and poly (L-lactic-co-glycolic acid) degradable polymer sponges attenuate astrocyte response and lesion growth in acute traumatic brain injury. Tissue Engineering 2007; 13(10) 2515-23.

[99] King VR, Alovskaya A, Wei DYT, Brown RA, Priestley J V. The use of injectable forms of fibrin and fibronectin to support axonal ingrowth after spinal cord injury. Biomaterials 2010; 31(15) 4447-56.

[100] Khaing ZZ, Milman BD, Vanscoy JE, Seidlits SK, Grill RJ, Schmidt CE. High molecular weight hyaluronic acid limits astrocyte activation and scar formation after spinal cord injury. Journal of Neural Engineering 2011; 8(4) 046033.

[101] Stokols S, Tuszynski MH. Freeze-dried agarose scaffolds with uniaxial channels stimulate and guide linear axonal growth following spinal cord injury. Biomaterials 2006; 27(3) 443-51.

[102] Chvatal SA, Kim Y-T, Bratt-Leal AM, Lee H, Bellamkonda R V. Spatial distribution and acute anti-inflammatory effects of Methylprednisolone after sustained local delivery to the contused spinal cord. Biomaterials 2008; 29(12) 1967-75.

[103] Fornaro M, Plescia J, Chheang S, Tallini G, Zhu Y-M, King M, et al. Fibronectin protects prostate cancer cells from tumor necrosis factor-alpha-induced apoptosis 
via the AKT/survivin pathway. The Journal of Biological Chemistry 2003; 278(50) 50402-11.

[104] Taylor L, Jones L, Tuszynski MH, Blesch A. Neurotrophin-3 gradients established by lentiviral gene delivery promote short-distance axonal bridging beyond cellular grafts in the injured spinal cord. The Journal of Neuroscience: The Official Journal of the Society for Neuroscience 2006; 26(38) 9713-21.

[105] Liu T, Houle JD, Xu J, Chan BP, Chew SY. Nanofibrous collagen nerve conduits for spinal cord repair. Tissue Engineering Part A 2012; 18(9-10) 1057-66.

[106] Stokols S, Tuszynski MH. The fabrication and characterization of linearly oriented nerve guidance scaffolds for spinal cord injury. Biomaterials 2004; 25(27) 583946.

[107] Prang P, Müller R, Eljaouhari A, Heckmann K, Kunz W, Weber T, et al. The promotion of oriented axonal regrowth in the injured spinal cord by alginate-based anisotropic capillary hydrogels. Biomaterials 2006; 27(19) 3560-9.

[108] Tysseling-Mattiace VM, Sahni V, Niece KL, Birch D, Czeisler C, Fehlings MG, et al. Self-assembling nanofibers inhibit glial scar formation and promote axon elongation after spinal cord injury. The Journal of Neuroscience 2008; 28(14) 3814-23.

[109] Cigognini D, Satta A, Colleoni B, Silva D, Donegà M, Antonini S, et al. Evaluation of early and late effects into the acute spinal cord injury of an injectable functionalized self-assembling scaffold. PloS One 2011; 6(5) e19782.

[110] Tsai EC, Dalton PD, Shoichet MS, Tator CH. Matrix inclusion within synthetic hydrogel guidance channels improves specific supraspinal and local axonal regeneration after complete spinal cord transection. Biomaterials 2006; 27(3) 519-33.

[111] Yao L, Damodaran G, Nikolskaya N, Gorman AM, Windebank A, Pandit A. The effect of laminin peptide gradient in enzymatically cross-linked collagen scaffolds on neurite growth. Journal of Biomedical Materials Research Part A 2010; 92(2) 484-92.

[112] Johnson PJ, Tatara A, Shiu A, Sakiyama-Elbert SE. Controlled release of neurotrophin-3 and platelet-derived growth factor from fibrin scaffolds containing neural progenitor cells enhances survival and differentiation into neurons in a subacute model of SCI. Cell Transplantation 2010; 19(1) 89-101.

[113] Patist CM, Mulder MB, Gautier SE, Maquet V, Jérôme R, Oudega M. Freeze-dried poly(D,L-lactic acid) macroporous guidance scaffolds impregnated with brainderived neurotrophic factor in the transected adult rat thoracic spinal cord. Biomaterials 2004; 25(9) 1569-82.

[114] Hurtado A, Moon LDF, Maquet V, Blits B, Jérôme R, Oudega M. Poly (D,L-lactic acid) macroporous guidance scaffolds seeded with Schwann cells genetically modified to secrete a bi-functional neurotrophin implanted in the completely transected adult rat thoracic spinal cord. Biomaterials 2006; 27(3) 430-42.

[115] Hurtado A, Cregg JM, Wang HB, Wendell DF, Oudega M, Gilbert RJ, et al. Robust CNS regeneration after complete spinal cord transection using aligned poly-Llactic acid microfibers. Biomaterials 2011; 32(26) 6068-79.

[116] Yang Y, De Laporte L, Zelivyanskaya ML, Whittlesey KJ, Anderson AJ, Cummings $\mathrm{BJ}$, et al. Multiple channel bridges for spinal cord injury: cellular characterization of host response. Tissue Engineering Part A 2009; 15(11) 3283-95.

[117] Lowry N, Goderie SK, Lederman P, Charniga C, Gooch MR, Gracey KD, et al. The effect of long-term release of Shh from implanted biodegradable microspheres on recovery from spinal cord injury in mice. Biomaterials 2012; 33(10) 2892-901.

[118] Moore MJ, Friedman JA, Lewellyn EB, Mantila SM, Krych AJ, Ameenuddin S, et al. Multiple-channel scaffolds to promote spinal cord axon regeneration. Biomaterials 2006; 27(3) 419-29. 
[119] Tuinstra HM, Aviles MO, Shin S, Holland SJ, Zelivyanskaya ML, Fast AG, et al. Multifunctional, multichannel bridges that deliver neurotrophin encoding lentivirus for regeneration following spinal cord injury. Biomaterials 2012; 33(5) 1618-26.

[120] Olson HE, Rooney GE, Gross L, Nesbitt JJ, Galvin KE, Knight A, et al. Neural stem cell- and Schwann cell-loaded biodegradable polymer scaffolds support axonal regeneration in the transected spinal cord. Tissue Engineering Part A 2009; 15(7) 1797-805.

[121] Chen BK, Knight AM, Madigan NN, Gross L, Dadsetan M, Nesbitt JJ, et al. Comparison of polymer scaffolds in rat spinal cord: a step toward quantitative assessment of combinatorial approaches to spinal cord repair. Biomaterials 2011; 32(32) 8077-86.

[122] Gelain F, Panseri S, Antonini S, Cunha C, Donega M, Lowery J, et al. Transplantation of nanostructured composite scaffolds results in the regeneration of chronically injured spinal cords. ACS Nano 2011; 5(1) 227-36.

[123] Meyer K, Palmer JW. The polysaccharide of the vitreous humor. J Biol Chem 1934; 107(3) 629-34.

[124] Salustri A, Fulop C. Role of hyaluronan during ovulation and maduration. http://www.glycoforum.gr.jp/science/hyaluronan/HA03/HA03E.html 1998; 1-12.

[125] Kogan G, Soltés L, Stern R, Gemeiner P. Hyaluronic acid: a natural biopolymer with a broad range of biomedical and industrial applications. Biotechnology Letters 2007; 29(1) 17-25.

[126] Laurent TC. The amorphous X-ray diffractogram of hyaluronic acid. Ark Kemi 1957; 11 513-8.

[127] Blundell CD, Reed MAC, Almond A. Complete assignment of hyaluronan oligosaccharides up to hexasaccharides. Carbohydrate Research 2006; 341(17) 2803-15.

[128] Laurent TC. The interaction between polysaccharides and other macromolecules. 9. The exclusion of molecules from hyaluronic acid gels and solutions. The Biochemical Journal 1964; 93(1) 106-12.

[129] Scott JE. Secondary structures in hyaluronan solutions: chemical and biological implications. Ciba Foundation Symposium 1989; 143 6-15; discussion 15-20, 281-5.

[130] Cowman MK, Matsuoka S. Experimental approaches to hyaluronan structure. Carbohydrate Research 2005; 340(5) 791-809.

[131] Cowman MK, Matsuoka S. The Intrinsic Viscosity of Hyaluronan. In: Kennedy JF, Phillips GO, William PA, editors. Hyaluronan, Cambridge (UK): Woodhead Pubishing; 2002, p. 75-8.

[132] Berriaud N, Milas M, Rinaudo M. Rheological study on mixtures of different molecular weight hyaluronates. International Journal of Biological Macromolecules 1994; 16(3) 137-42.

[133] Fouissac E, Milas M, Rinaudo M, Borsali R. Influence of the ionic strength on the dimensions of sodium hyaluronate. Macromolecules 1992; 25(21) 5613-7.

[134] Balazs EA. The Physical Properties of Synovial Fluid and the Special Role of Hyaluronic Acid. In: Helfet A, editor. Disorders of the knee, Philadelphia (PA): T.B. Lippincott Company; 1974, p. 63-75.

[135] Hardingham T. Solution properties of hyaluronan. In: Garg HG, Hales CA, editors. Chemistry and Biology of Hyaluronan, Boston (MA): Elsevier; 2004, p. 1-19.

[136] Cowman MK, Mendichi R. Methods for determination of hyaluronan molecular weight. In: Garg HG, Hales CA, editors. Chemistry and Biology of Hyaluronan, Boston (MA): Elsevier; 2004, p. 41-55.

[137] Bothner $\mathrm{H}$, Waaler T, Wik O. Limiting viscosity number and weight average molecular weight of hyaluronate samples produced by heat degradation. International Journal of Biological Macromolecules 1988; 10(5) 287-91. 
[138] Tokita Y, Okamoto A. Hydrolytic degradation of hyaluronic acid. Polymer Degradation and Stability 1995; 48(2) 269-73.

[139] Tømmeraas K, Melander C. Kinetics of hyaluronan hydrolysis in acidic solution at various $\mathrm{pH}$ values. Biomacromolecules 2008; 9(6) 1535-40.

[140] Inoue Y, Nagasawa K. Preparation, by chemical degradation of hyaluronic acid, of a series of even- and odd-numbered oligosaccharides having a 2-acetamido-2deoxy-d-glucose and a d-glucuronic acid residue, respectively, at the reducing end. Carbohydrate Research 1985; 141(1) 99-110.

[141] Reháková M, Bakos D, Soldán M, Vizárová K. Depolymerization reactions of hyaluronic acid in solution. International Journal of Biological Macromolecules 1994; 16(3) 121-4.

[142] Vercruysse KP, Lauwers AR, Demeester JM. Absolute and empirical determination of the enzymatic activity and kinetic investigation of the action of hyaluronidase on hyaluronan using viscosimetry. The Biochemical Journal 1995; 306(Pt 1) 153-60.

[143] Orviský E, Šoltés L, Chabreček P, Novák I, Stančíková M. Size exclusion chromatographic characterization of sodium hyaluronate fractions prepared by high energetic sonication. Chromatographia 1993; 37(1-2) 20-2.

[144] Drímalová E, Velebný V, Sasinková V, Hromádková Z, Ebringerová A. Degradation of hyaluronan by ultrasonication in comparison to microwave and conventional heating. Carbohydrate Polymers 2005; 61(4) 420-6.

[145] Stern R, Kogan G, Jedrzejas MJ, Soltés L. The many ways to cleave hyaluronan. Biotechnology Advances 2007; 25(6) 537-57.

[146] McNeil JD, Wiebkin OW, Betts WH, Cleland LG. Depolymerisation products of hyaluronic acid after exposure to oxygen-derived free radicals. Annals of the Rheumatic Diseases 1985; 44(11) 780-9.

[147] Lawwill T. Three major pathologic processes caused by light in the primate retina: a search for mechanisms. Transactions of the American Ophthalmological Society 1982; 80 517-79.

[148] Bezáková Z, Hermannová M, Drímalová E, Malovíková A, Ebringerová A, Velebný $V$. Effect of microwave irradiation on the molecular and structural properties of hyaluronan. Carbohydrate Polymers 2008; 73(4) 640-6.

[149] Lapcík L, Chabrecek P, Stasko A. Photodegradation of hyaluronic acid: EPR and size exclusion chromatography study. Biopolymers 1991; 31(12) 1429-35.

[150] Srinivas A, Ramamurthi A. Effects of gamma-irradiation on physical and biologic properties of crosslinked hyaluronan tissue engineering scaffolds. Tissue Engineering 2007; 13(3) 447-59.

[151] Galema SA. Microwave chemistry. Chemical Society Reviews 1997; 26(3) 233.

[152] Meyer K, Dubos R, Smith EM. The hydrolysis of the polysaccharide acids of vitreous humor, of umbilical cord, and of streptococcus by the autolytic enzyme of pneumococcus. J Biol Chem 1937; 118 71-8.

[153] Girish KS, Kemparaju K. The magic glue hyaluronan and its eraser hyaluronidase: a biological overview. Life Sciences 2007; 80(21) 1921-43.

[154] Al'Qteishat A, Gaffney J, Krupinski J, Rubio F, West D, Kumar S, et al. Changes in hyaluronan production and metabolism following ischaemic stroke in man. Brain : A Journal of Neurology 2006; 129(Pt 8) 2158-76.

[155] Makris G, Wright JD, Ingham E, Holland KT. The hyaluronate lyase of Staphylococcus aureus - a virulence factor? Microbiology 2004; 150(Pt 6) 200513.

[156] Ponnuraj K, Jedrzejas MJ. Mechanism of hyaluronan binding and degradation: structure of Streptococcus pneumoniae hyaluronate lyase in complex with hyaluronic acid disaccharide at $1.7 \mathrm{~A}$ resolution. Journal of Molecular Biology 2000; 299(4) 885-95. 
[157] Stern R, Jedrzejas MJ. Hyaluronidases: their genomics, structures, and mechanisms of action. Chemical Reviews 2006; 106(3) 818-39.

[158] Csóka AB, Frost GI, Wong T, Stern R, Csóka TB. Purification and microsequencing of hyaluronidase isozymes from human urine. FEBS Letters 1997; 417(3) 307-10.

[159] Lepperdinger G, Müllegger J, Kreil G. Hyal2--less active, but more versatile? Matrix Biology : Journal of the International Society for Matrix Biology 2001; 20(8) 509-14.

[160] Myles DG, Primakoff P. Why did the sperm cross the cumulus? To get to the oocyte. Functions of the sperm surface proteins $\mathrm{PH}-20$ and fertilin in arriving at, and fusing with, the egg. Biology of Reproduction 1997; 56(2) 320-7.

[161] Chen F, Kakizaki I, Yamaguchi M, Kojima K, Takagaki K, Endo M. Novel products in hyaluronan digested by bovine testicular hyaluronidase. Glycoconjugate Journal 2009; 26(5) 559-66.

[162] Nicoll SB, Barak O, Csóka AB, Bhatnagar RS, Stern R. Hyaluronidases and CD44 undergo differential modulation during chondrogenesis. Biochemical and Biophysical Research Communications 2002; 292(4) 819-25.

[163] Kaneiwa T, Miyazaki A, Kogawa R, Mizumoto S, Sugahara K, Yamada S. Identification of amino acid residues required for the substrate specificity of human and mouse chondroitin sulfate hydrolase (conventional hyaluronidase-4). The Journal of Biological Chemistry 2012; 287(50) 42119-28.

[164] Miller KA, Shao M, Martin-DeLeon PA. Hyalp1 in murine sperm function: evidence for unique and overlapping functions with other reproductive hyaluronidases. Journal of Andrology 2007; 28(1) 67-76.

[165] Weigel PH, Hascall VC, Tammi M. Hyaluronan synthases. The Journal of Biological Chemistry 1997; 272(22) 13997-4000.

[166] Weigel PH. The hyaluronan shyntases. In: Garg HG, Hales CA, editors. Chemistry and Biology of Hyaluronan, Boston (MA): Elsevier; 2004, p. 553-64.

[167] Jiang D, Liang J, Noble PW. Hyaluronan in tissue injury and repair. Annual Review of Cell and Developmental Biology 2007; 23 435-61.

[168] Seyfried NT, Day AJ, Almond A. Experimental evidence for all-or-none cooperative interactions between the G1-domain of versican and multivalent hyaluronan oligosaccharides. Matrix Biology : Journal of the International Society for Matrix Biology 2006; 25(1) 14-9.

[169] Rauch U, Karthikeyan L, Maurel P, Margolis RU, Margolis RK. Cloning and primary structure of neurocan, a developmentally regulated, aggregating chondroitin sulfate proteoglycan of brain. The Journal of Biological Chemistry 1992; 267(27) 19536-47.

[170] Knudson CB, Knudson W. Hyaluronan and CD44: modulators of chondrocyte metabolism. Clinical Orthopaedics and Related Research 2004; (427 Suppl) S152-62.

[171] Toole BP. Hyaluronan: from extracellular glue to pericellular cue. Nature Reviews Cancer 2004; 4(7) 528-39.

[172] Knudson W, Chow G, Knudson CB. CD44-mediated uptake and degradation of hyaluronan. Matrix Biology : Journal of the International Society for Matrix Biology 2002; 21(1) 15-23.

[173] Toole BP. Hyaluronan in morphogenesis. Seminars in Cell \& Developmental Biology 2001; 12(2) 79-87.

[174] Entwistle J, Zhang S, Yang B, Wong C, Li Q, Hall CL, et al. Characterization of the murine gene encoding the hyaluronan receptor RHAMM. Gene 1995; 163(2) 233-8.

[175] Savani RC, Cao G, Pooler PM, Zaman A, Zhou Z, DeLisser HM. Differential involvement of the hyaluronan (HA) receptors CD44 and receptor for HA-mediated 
motility in endothelial cell function and angiogenesis. The Journal of Biological Chemistry 2001; 276(39) 36770-8.

[176] Turley EA, Noble PW, Bourguignon LYW. Signaling properties of hyaluronan receptors. The Journal of Biological Chemistry 2002; 277(7) 4589-92.

[177] Zhang S, Chang MC, Zylka D, Turley S, Harrison R, Turley EA. The hyaluronan receptor RHAMM regulates extracellular-regulated kinase. The Journal of Biological Chemistry 1998; 273(18) 11342-8.

[178] Zaman A, Cui Z, Foley JP, Zhao H, Grimm PC, Delisser HM, et al. Expression and role of the hyaluronan receptor RHAMM in inflammation after bleomycin injury. American Journal of Respiratory Cell and Molecular Biology 2005; 33(5) 447-54.

[179] Sherman L, Sleeman J, Herrlich P, Ponta H. Hyaluronate receptors: key players in growth, differentiation, migration and tumor progression. Current Opinion in Cell Biology 1994; 6(5) 726-33.

[180] Borowsky ML, Hynes RO. Layilin, a novel talin-binding transmembrane protein homologous with $\mathrm{C}$-type lectins, is localized in membrane ruffles. The Journal of Cell Biology 1998; 143(2) 429-42.

[181] Wegener KL, Basran J, Bagshaw CR, Campbell ID, Roberts GCK, Critchley DR, et al. Structural basis for the interaction between the cytoplasmic domain of the hyaluronate receptor layilin and the talin F3 subdomain. Journal of Molecular Biology 2008; 382(1) 112-26.

[182] Zhou B, Weigel JA, Fauss L, Weigel PH. Identification of the hyaluronan receptor for endocytosis (HARE). The Journal of Biological Chemistry 2000; 275(48) 37733-41.

[183] Jackson DG. Biology of the lymphatic marker LYVE-1 and applications in research into lymphatic trafficking and lymphangiogenesis. APMIS: Acta Pathologica, Microbiologica, et Immunologica Scandinavica n.d.; 112(7-8) 526-38.

[184] Poltorak A, He X, Smirnova I, Liu MY, Van Huffel C, Du X, et al. Defective LPS signaling in $\mathrm{C} 3 \mathrm{H} / \mathrm{HeJ}$ and $\mathrm{C} 57 \mathrm{BL} / 10 \mathrm{ScCr}$ mice: mutations in Tlr4 gene. Science (New York, NY) 1998; 282(5396) 2085-8.

[185] Takeuchi O, Hoshino K, Kawai T, Sanjo H, Takada H, Ogawa T, et al. Differential roles of TLR2 and TLR4 in recognition of gram-negative and gram-positive bacterial cell wall components. Immunity 1999; 11(4) 443-51.

[186] Xu Y, Jagannath C, Liu X-D, Sharafkhaneh A, Kolodziejska KE, Eissa NT. Tolllike receptor 4 is a sensor for autophagy associated with innate immunity. Immunity 2007; 27(1) 135-44.

[187] Texereau J, Chiche J-D, Taylor W, Choukroun G, Comba B, Mira J-P. The importance of Toll-like receptor 2 polymorphisms in severe infections. Clinical Infectious Diseases: An Official Publication of the Infectious Diseases Society of America 2005; 41(Suppl 7) S408-15.

[188] Vigetti D, Karousou E, Viola M, Deleonibus S, De Luca G, Passi A. Hyaluronan: Biosynthesis and signaling. Biochimica et Biophysica Acta 2014; 1840(8) 2452-9.

[189] Stern R, Asari AA, Sugahara KN. Hyaluronan fragments: an information-rich system. European Journal of Cell Biology 2006; 85(8) 699-715.

[190] West DC, Kumar S. The effect of hyaluronate and its oligosaccharides on endothelial cell proliferation and monolayer integrity. Experimental Cell Research 1989; 183(1) 179-96.

[191] Nyström B, Kjøniksen A-L, Beheshti N, Maleki A, Zhu K, Knudsen KD, et al. Characterization of polyelectrolyte features in polysaccharide systems and mucin. Advances in Colloid and Interface Science 2010; 158(1-2) 108-18.

[192] Nakajima N, Ikada Y. Mechanism of amide formation by carbodiimide for bioconjugation in aqueous media. Bioconjugate Chemistry 1995; 6(1) 123-30.

[193] Bulpitt P, Aeschlimann D. New strategy for chemical modification of hyaluronic acid: preparation of functionalized derivatives and their use in the formation of 
novel biocompatible hydrogels. Journal of Biomedical Materials Research 1999; 47(2) 152-69.

[194] Matsuda T, Magoshi T. Preparation of vinylated polysaccharides and photofabrication of tubular scaffolds as potential use in tissue engineering. Biomacromolecules 2002; 3(5) 942-50.

[195] Shoham N, Sasson AL, Lin F-H, Benayahu D, Haj-Ali R, Gefen A. The mechanics of hyaluronic acid/adipic acid dihydrazide hydrogel: towards developing a vessel for delivery of preadipocytes to native tissues. Journal of the Mechanical Behavior of Biomedical Materials 2013; 28 320-31.

[196] Huang B, Li C-Q, Zhou Y, Luo G, Zhang C-Z. Collagen II/hyaluronan/chondroitin6-sulfate tri-copolymer scaffold for nucleus pulposus tissue engineering. Journal of Biomedical Materials Research Part B, Applied Biomaterials 2010; 92(2) 32231.

[197] Xu X, Jha AK, Harrington DA, Farach-Carson MC, Jia X. Hyaluronic Acid-Based Hydrogels: from a Natural Polysaccharide to Complex Networks. Soft Matter 2012; 8(12) 3280-94.

[198] Schneider A, Picart C, Senger B, Schaaf P, Voegel J-C, Frisch B. Layer-by-layer films from hyaluronan and amine-modified hyaluronan. Langmuir: The ACS Journal of Surfaces and Colloids 2007; 23(5) 2655-62.

[199] Moriyama K, Ooya T, Yui N. Hyaluronic acid grafted with poly(ethylene glycol) as a novel peptide formulation. Journal of Controlled Release : Official Journal of the Controlled Release Society 1999; 59(1) 77-86.

[200] Luo Y, Prestwich GD. Synthesis and selective cytotoxicity of a hyaluronic acidantitumor bioconjugate. Bioconjugate Chemistry n.d.; 10(5) 755-63.

[201] Luo Y, Bernshaw NJ, Lu Z-R, Kopecek J, Prestwich GD. Targeted delivery of doxorubicin by HPMA copolymer-hyaluronan bioconjugates. Pharmaceutical Research 2002; 19(4) 396-402.

[202] Magnani A, Rappuoli R, Lamponi S, Barbucci R. Novel polysaccharide hydrogels: characterization and properties. Polymers for Advanced Technologies 2000; 11 (812) 488-95.

[203] Della Valle F, Romeo A. Crosslinked carboxy polysaccharides. US5676964 A, 1997.

[204] Young J-J, Cheng K-M, Tsou T-L, Liu H-W, Wang H-J. Preparation of cross-linked hyaluronic acid film using 2-chloro-1-methylpyridinium iodide or water-soluble 1ethyl-(3,3-dimethylaminopropyl)carbodiimide. Journal of Biomaterials Science Polymer Edition 2004; 15(6) 767-80.

[205] Bergman K, Elvingson C, Hilborn J, Svensk G, Bowden T. Hyaluronic acid derivatives prepared in aqueous media by triazine-activated amidation. Biomacromolecules 2007; 8(7) 2190-5.

[206] Bellini D, Topai A. Amides of hyaluronic acid and the derivatives thereof and a process for their preparation. CA2339066 A1, 2000.

[207] D'Este M, Alini M, Eglin D. Single step synthesis and characterization of thermoresponsive hyaluronan hydrogels. Carbohydrate Polymers 2012; 90(3) 1378-85.

[208] Picotti F, Fabbian M, Gianni R, Sechi A, Stucchi L, Bosco M. Hyaluronic acid lipoate: synthesis and physicochemical properties. Carbohydrate Polymers 2013; 93(1) 273-8.

[209] Pelletier S, Hubert P, Payan E, Marchal P, Choplin L, Dellacherie E. Amphiphilic derivatives of sodium alginate and hyaluronate for cartilage repair: rheological properties. Journal of Biomedical Materials Research 2001; 54(1) 102-8.

[210] Huin-Amargier C, Marchal P, Payan E, Netter P, Dellacherie E. New physically and chemically crosslinked hyaluronate (HA)-based hydrogels for cartilage repair. Journal of Biomedical Materials Research Part A 2006; 76(2) 416-24. 
[211] Della Valle F, Romeo A. Esters of hyaluronic acid and their salts. EP0216453 A2, 1987.

[212] Bencherif SA, Srinivasan A, Horkay F, Hollinger JO, Matyjaszewski K, Washburn NR. Influence of the degree of methacrylation on hyaluronic acid hydrogels properties. Biomaterials 2008; 29(12) 1739-49.

[213] Baier Leach J, Bivens KA, Patrick CW, Schmidt CE. Photocrosslinked hyaluronic acid hydrogels: natural, biodegradable tissue engineering scaffolds. Biotechnology and Bioengineering 2003; 82(5) 578-89.

[214] Jin Y, Yamanaka J, Sato S, Miyata I, Yomota C, Yonese M. Recyclable characteristics of hyaluronate-polyhydroxyethyl acrylate blend hydrogel for controlled releases. Journal of Controlled Release: Official Journal of the Controlled Release Society 2001; 73(2-3) 173-81.

[215] Li X, Luan S, Shi H, Yang H, Song L, Jin J, et al. Improved biocompatibility of poly (styrene-b-(ethylene-co-butylene)-b-styrene) elastomer by a surface graft polymerization of hyaluronic acid. Colloids and Surfaces B, Biointerfaces 2013; $102210-7$.

[216] De Nooy AE, Capitani D, Masci G, Crescenzi V. Ionic polysaccharide hydrogels via the Passerini and Ugi multicomponent condensations: synthesis, behavior and solid-state NMR characterization. Biomacromolecules 2000; 1(2) 259-67.

[217] Crescenzi V, Francescangeli A, Capitani D, Mannina L, Renier D, Bellini D. Hyaluronan networking via Ugi's condensation using lysine as cross-linker diamine. Carbohydrate Polymers 2003; 53(3) 311-6.

[218] Schanté CE, Zuber G, Herlin C, Vandamme TF. Chemical modifications of hyaluronic acid for the synthesis of derivatives for a broad range of biomedical applications. Carbohydrate Polymers 2011; 85(3) 469-89.

[219] Fakhari A, Berkland C. Applications and emerging trends of hyaluronic acid in tissue engineering, as a dermal filler and in osteoarthritis treatment. Acta Biomaterialia 2013; 9(7) 7081-92.

[220] Ramamurthi A, Vesely I. Smooth muscle cell adhesion on crosslinked hyaluronan gels. Journal of Biomedical Materials Research 2002; 60(1) 195-205.

[221] Collins MN, Birkinshaw C. Comparison of the effectiveness of four different crosslinking agents with hyaluronic acid hydrogel films for tissue-culture applications. Journal of Applied Polymer Science 2007; 104(5) 3183-91.

[222] Oh EJ, Kang S-W, Kim B-S, Jiang G, Cho IH, Hahn SK. Control of the molecular degradation of hyaluronic acid hydrogels for tissue augmentation. Journal of Biomedical Materials Research Part A 2008; 86(3) 685-93.

[223] Balazs EA, Leshchiner A. Cross-linked gels of hyaluronic acid and products containing such gels. DE 3520008 A1, 1986.

[224] Kimmelman CP, Edelstein DR, Cheng HJ. Sepragel sinus (hylan B) as a postsurgical dressing for endoscopic sinus surgery. Otolaryngology--Head and Neck Surgery : Official Journal of American Academy of Otolaryngology-Head and Neck Surgery 2001; 125(6) 603-8.

[225] Zhang J, Ma X, Hui J, Ma P, Deng J, Fan D. Crosslinking of hyaluronic acid and human-like collagen with divinyl sulfone. Journal of Chemical and Pharmaceutical Research 2014; 6(1) 726-30.

[226] Parent EG, Larsen NE. Hydrophilic biopolymer-drug conjugates, their preparation and use. US 20040087488 A1, 2004.

[227] Serban MA, Yang G, Prestwich GD. Synthesis, characterization and chondroprotective properties of a hyaluronan thioethyl ether derivative. Biomaterials 2008; 29(10) 1388-99.

[228] Tomihata K, Ikada Y. Crosslinking of hyaluronic acid with glutaraldehyde. Journal of Polymer Science Part A: Polymer Chemistry 1997; 35(16) 3553-9.

[229] Crescenzi V, Francescangeli A, Taglienti A, Capitani D, Mannina L. Synthesis and partial characterization of hydrogels obtained via glutaraldehyde crosslinking of 
acetylated chitosan and of hyaluronan derivatives. Biomacromolecules 2003; 4(4) 1045-54.

[230] Ramires PA, Milella E. Biocompatibility of poly(vinyl alcohol)-hyaluronic acid and poly(vinyl alcohol)-gellan membranes crosslinked by glutaraldehyde vapors. Journal of Materials Science Materials in Medicine 2002; 13(1) 119-23.

[231] Mlcochová P, Bystrický S, Steiner B, Machová E, Koós M, Velebný V, et al. Synthesis and characterization of new biodegradable hyaluronan alkyl derivatives. Biopolymers 2006; 82(1) 74-9.

[232] Chytil M, Pekař M. Effect of new hydrophobic modification of hyaluronan on its solution properties: evaluation of self-aggregation. Carbohydrate Polymers 2009; 76(3) 443-8.

[233] Pravata L, Braud C, Boustta M, El Ghzaoui A, Tømmeraas K, Guillaumie F, et al. New amphiphilic lactic acid oligomer-hyaluronan conjugates: synthesis and physicochemical characterization. Biomacromolecules 2008; 9(1) 340-8.

[234] Toemmeraas K, Eenschooten C. Aryl/alkyl succinic anhydride hyaluronan derivatives. WO2007033677 A1, 2007.

[235] Eenschooten C, Guillaumie F, Kontogeorgis GM, Stenby EH, Schwach-Abdellaoui K. Preparation and structural characterisation of novel and versatile amphiphilic octenyl succinic anhydride-modified hyaluronic acid derivatives. Carbohydrate Polymers 2010; 79(3) 597-605.

[236] Neves-Petersen MT, Klitgaard S, Skovsen E, Petersen SB, Tømmeraas K, Schwach-Abdellaoui K. Biophysical properties of phenyl succinic acid derivatised hyaluronic acid. Journal of Fluorescence 2010; 20(2) 483-92.

[237] Smeds KA, Pfister-Serres A, Hatchell DL, Grinstaff MW. Synthesis of a novel polysaccharide hydrogel. Journal of Macromolecular Science, Part A 1999; 36(78) 981-9.

[238] Burdick JA, Chung C, Jia X, Randolph MA, Langer R. Controlled degradation and mechanical behavior of photopolymerized hyaluronic acid networks. Biomacromolecules 2005; 6(1) 386-91.

[239] Brigham MD, Bick A, Lo E, Bendali A, Burdick JA, Khademhosseini A. Mechanically robust and bioadhesive collagen and photocrosslinkable hyaluronic acid semi-interpenetrating networks. Tissue Engineering Part A 2009; 15(7) 164553.

[240] Pitarresi G, Pierro P, Palumbo FS, Tripodo G, Giammona G. Photo-cross-linked hydrogels with polysaccharide-poly(amino acid) structure: new biomaterials for pharmaceutical applications. Biomacromolecules 2006; 7(4) 1302-10.

[241] Sahoo S, Chung C, Khetan S, Burdick JA. Hydrolytically degradable hyaluronic acid hydrogels with controlled temporal structures. Biomacromolecules 2008; 9(4) 1088-92.

[242] Bae KH, Yoon JJ, Park TG. Fabrication of hyaluronic acid hydrogel beads for cell encapsulation. Biotechnology Progress 2006; 22(1) 297-302.

[243] Miki D, Dastgheib K, Kim T, Pfister-Serres A, Smeds KA, Inoue M, et al. A photopolymerized sealant for corneal lacerations. Cornea 2002; 21(4) 393-9.

[244] Crescenzi V, Francescangeli A, Segre AL, Capitani D, Mannina L, Renier D, et al. NMR structural study of hydrogels based on partially deacetylated hyaluronan. Macromolecular Bioscience 2002; 2(6) 272-9.

[245] Oerther S, Maurin AC, Payan E, Hubert P, Lapicque F, Presle N, et al. High interaction alginate-hyaluronate associations by hyaluronate deacetylation for the preparation of efficient biomaterials. Biopolymers 2000; 54(4) 273-81.

[246] Jia X, Colombo G, Padera R, Langer R, Kohane DS. Prolongation of sciatic nerve blockade by in situ cross-linked hyaluronic acid. Biomaterials 2004; 25(19) 4797804. 
[247] Jha AK, Hule RA, Jiao T, Teller SS, Clifton RJ, Duncan RL, et al. Structural Analysis and Mechanical Characterization of Hyaluronic Acid-Based Doubly Cross-Linked Networks. Macromolecules 2009; 42(2) 537-46.

[248] Liu L-S, Ng C-K, Thompson AY, Poser JW, Spiro RC. Hyaluronate-heparin conjugate gels for the delivery of basic fibroblast growth factor (FGF-2). Journal of Biomedical Materials Research 2002; 62(1) 128-35.

[249] Glass JR, Dickerson KT, Stecker K, Polarek JW. Characterization of a hyaluronic acid-Arg-Gly-Asp peptide cell attachment matrix. Biomaterials 1996; 17(11) 11018.

[250] Shu XZ, Liu Y, Luo Y, Roberts MC, Prestwich GD. Disulfide cross-linked hyaluronan hydrogels. Biomacromolecules 2002; 3(6) 1304-11.

[251] Kim KS, Park SJ, Yang J-A, Jeon J-H, Bhang SH, Kim B-S, et al. Injectable hyaluronic acid-tyramine hydrogels for the treatment of rheumatoid arthritis. Acta Biomaterialia 2011; 7(2) 666-74.

[252] Asayama S, Nogawa M, Takei Y, Akaike T, Maruyama A. Synthesis of novel polyampholyte comb-type copolymers consisting of a poly(L-lysine) backbone and hyaluronic acid side chains for a DNA carrier. Bioconjugate Chemistry 1998; 9(4) 476-81.

[253] Intergel. FDA premarket approval letter P990015 for gynecare intergel, November 16, 2001. Lifecore Biomedical I, ed., Chaska, MN, 2001 n.d.;

[254] Polexe RC, Delair T. Elaboration of stable and antibody functionalized positively charged colloids by polyelectrolyte complexation between chitosan and hyaluronic acid. Molecules (Basel, Switzerland) 2013; 18(7) 8563-78.

[255] Li H, Jiang J, Ge Y, Xu J, Zhang P, Zhong W, et al. Layer-by-layer hyaluronic acidchitosan coating promoted new collagen ingrowth into a poly(ethylene terephthalate) artificial ligament in a rabbit medical collateral ligament (MCL) reconstruction model. Journal of Biomaterials Science Polymer Edition 2013; 24(4) 431-46.

[256] Kim SJ, Shin SR, Lee KB, Park YD, Kim SI. Synthesis and characteristics of polyelectrolyte complexes composed of chitosan and hyaluronic acid. Journal of Applied Polymer Science 2004; 91(5) 2908-13.

[257] Luo Y, Wang Q. Recent development of chitosan-based polyelectrolyte complexes with natural polysaccharides for drug delivery. International Journal of Biological Macromolecules 2014; 64 353-67.

[258] Oh EJ, Park K, Kim KS, Kim J, Yang J-A, Kong J-H, et al. Target specific and longacting delivery of protein, peptide, and nucleotide therapeutics using hyaluronic acid derivatives. Journal of Controlled Release : Official Journal of the Controlled Release Society 2010; 141(1) 2-12.

[259] Kisiel M, Klar AS, Ventura M, Buijs J, Mafina M-K, Cool SM, et al. Complexation and sequestration of BMP-2 from an ECM mimetic hyaluronan gel for improved bone formation. PloS One 2013; 8(10) e78551.

[260] Jeong Y-I, Kim S-T, Jin S-G, Ryu H-H, Jin Y-H, Jung T-Y, et al. Cisplatinincorporated hyaluronic acid nanoparticles based on ion-complex formation. Journal of Pharmaceutical Sciences 2008; 97(3) 1268-76.

[261] Balazs EA. Viscoelastic properties of hyaluronan and its therapeutic use. In: Garg $\mathrm{HG}$, Hales CA, editors. Chemistry and Biology of Hyaluronan, Boston (MA): Elsevier; 2004, p. 415-43.

[262] Maltese A, Borzacchiello A, Mayol L, Bucolo C, Maugeri F, Nicolais L, et al. Novel polysaccharides-based viscoelastic formulations for ophthalmic surgery: rheological characterization. Biomaterials 2006; 27(29) 5134-42.

[263] Larsen NE, Pollak CT, Reiner K, Leshchiner E, Balazs EA. Hylan gel biomaterial: dermal and immunologic compatibility. Journal of Biomedical Materials Research 1993; 27(9) 1129-34. 
[264] Su W-Y, Chen K-H, Chen Y-C, Lee Y-H, Tseng C-L, Lin F-H. An injectable oxidated hyaluronic acid/adipic acid dihydrazide hydrogel as a vitreous substitute. Journal of Biomaterials Science Polymer Edition 2011; 22(13) 1777-97.

[265] Soltés L, Mendichi R, Kogan G, Schiller J, Stankovska M, Arnhold J. Degradative action of reactive oxygen species on hyaluronan. Biomacromolecules 2006; 7(3) 659-68.

[266] Chong BF, Blank LM, Mclaughlin R, Nielsen LK. Microbial hyaluronic acid production. Applied Microbiology and Biotechnology 2005; 66(4) 341-51.

[267] Greenberg DD, Stoker A, Kane S, Cockrell M, Cook JL. Biochemical effects of two different hyaluronic acid products in a co-culture model of osteoarthritis. Osteoarthritis and Cartilage / OARS, Osteoarthritis Research Society 2006; 14(8) 814-22.

[268] Jones D. Volumizing the face with soft tissue fillers. Clinics in Plastic Surgery 2011; 38(3) 379-90.

[269] Upton DC, Johnson M, Zelazny SK, Dailey SH. Prospective evaluation of officebased injection laryngoplasty with hyaluronic acid gel. The Annals of Otology, Rhinology, and Laryngology 2013; 122(9) 541-6.

[270] Lone F, Sultan AH, Thakar R. Long-term outcome of transurethral injection of hyaluronic acid/dextranomer (NASHA/Dx gel) for the treatment of stress urinary incontinence (SUI). International Urogynecology Journal 2010; 21(11) 1359-64.

[271] Narins RS, Brandt F, Leyden J, Lorenc ZP, Rubin M, Smith S. A randomized, double-blind, multicenter comparison of the efficacy and tolerability of Restylane versus Zyplast for the correction of nasolabial folds. Dermatologic Surgery: Official Publication for American Society for Dermatologic Surgery 2003; 29(6) 588-95.

[272] Hertegård S, Hallén L, Laurent C, Lindström E, Olofsson K, Testad P, et al. Crosslinked hyaluronan versus collagen for injection treatment of glottal insufficiency: 2year follow-up. Acta Oto-Laryngologica 2004; 124(10) 1208-14.

[273] Molteni G, Bergamini G, Ricci-Maccarini A, Marchese C, Ghidini A, AlicandriCiufelli $\mathrm{M}$, et al. Auto-crosslinked hyaluronan gel injections in phonosurgery. Otolaryngology--Head and Neck Surgery: Official Journal of American Academy of Otolaryngology-Head and Neck Surgery 2010; 142(4) 547-53.

[274] Van Kerrebroeck P, ter Meulen F, Larsson G, Farrelly E, Edwall L, Fianu-Jonasson A. Efficacy and safety of a novel system (NASHA/Dx copolymer using the Implacer device) for treatment of stress urinary incontinence. Urology 2004; 64(2) 276-81.

[275] Lightner D, Rovner E, Corcos J, Payne C, Brubaker L, Drutz H, et al. Randomized controlled multisite trial of injected bulking agents for women with intrinsic sphincter deficiency: mid-urethral injection of Zuidex via the Implacer versus proximal urethral injection of Contigen cystoscopically. Urology 2009; 74(4) 7715 .

[276] Edmonds M, Wilson M, Foster A. Diabetic foot ulcers. Nursing Standard 1999; 14(12) 39-45.

[277] Uccioli L, Giurato L, Ruotolo V, Ciavarella A, Grimaldi MS, Piaggesi A, et al. Twostep autologous grafting using HYAFF scaffolds in treating difficult diabetic foot ulcers: results of a multicenter, randomized controlled clinical trial with long-term follow-up. The International Journal of Lower Extremity Wounds 2011; 10(2) 805 .

[278] Collins MN, Birkinshaw C. Hyaluronic acid based scaffolds for tissue engineering-a review. Carbohydrate Polymers 2013; 92(2) 1262-79.

[279] Collins MN, Birkinshaw C. Morphology of crosslinked hyaluronic acid porous hydrogels. Journal of Applied Polymer Science 2011; 120(2) 1040-9.

[280] Wang T-W, Spector M. Development of hyaluronic acid-based scaffolds for brain tissue engineering. Acta Biomaterialia 2009; 5(7) 2371-84. 
[281] Davidenko N, Campbell JJ, Thian ES, Watson CJ, Cameron RE. Collagenhyaluronic acid scaffolds for adipose tissue engineering. Acta Biomaterialia 2010; 6(10) 3957-68.

[282] Suri S, Schmidt CE. Photopatterned collagen-hyaluronic acid interpenetrating polymer network hydrogels. Acta Biomaterialia 2009; 5(7) 2385-97.

[283] Zhang F, He C, Cao L, Feng W, Wang H, Mo X, et al. Fabrication of gelatinhyaluronic acid hybrid scaffolds with tunable porous structures for soft tissue engineering. International Journal of Biological Macromolecules 2011; 48(3) 47481.

[284] Tan H, Chu CR, Payne KA, Marra KG. Injectable in situ forming biodegradable chitosan-hyaluronic acid based hydrogels for cartilage tissue engineering. Biomaterials 2009; 30(13) 2499-506.

[285] Zhang L-M, Wu C-X, Huang J-Y, Peng X-H, Chen P, Tang S-Q. Synthesis and characterization of a degradable composite agarose/HA hydrogel. Carbohydrate Polymers 2012; 88(4) 1445-52.

[286] Hou S, Xu Q, Tian W, Cui F, Cai Q, Ma J, et al. The repair of brain lesion by implantation of hyaluronic acid hydrogels modified with laminin. Journal of Neuroscience Methods 2005; 148(1) 60-70.

[287] Cui FZ, Tian WM, Hou SP, Xu QY, Lee I-S. Hyaluronic acid hydrogel immobilized with RGD peptides for brain tissue engineering. Journal of Materials Science Materials in Medicine 2006; 17(12) 1393-401.

[288] Kisiel M, Martino MM, Ventura M, Hubbell JA, Hilborn J, Ossipov DA. Improving the osteogenic potential of BMP-2 with hyaluronic acid hydrogel modified with integrin-specific fibronectin fragment. Biomaterials 2013; 34(3) 704-12.

[289] Tian WM, Hou SP, Ma J, Zhang CL, Xu QY, Lee IS, et al. Hyaluronic acid-poly-Dlysine-based three-dimensional hydrogel for traumatic brain injury. Tissue Engineering 2005; 11(3-4) 513-25.

[290] Ananthanarayanan B, Kim Y, Kumar S. Elucidating the mechanobiology of malignant brain tumors using a brain matrix-mimetic hyaluronic acid hydrogel platform. Biomaterials 2011; 32(31) 7913-23.

[291] Hou S, Tian W, Xu Q, Cui F, Zhang J, Lu Q, et al. The enhancement of cell adherence and inducement of neurite outgrowth of dorsal root ganglia co-cultured with hyaluronic acid hydrogels modified with Nogo-66 receptor antagonist in vitro. Neuroscience 2006; 137(2) 519-29.

[292] Niiyama H, Kuroyanagi Y. Development of novel wound dressing composed of hyaluronic acid and collagen sponge containing epidermal growth factor and vitamin $C$ derivative. Journal of Artificial Organs: The Official Journal of the Japanese Society for Artificial Organs 2014; 17(1) 81-7.

[293] Lei Y, Huang S, Sharif-Kashani P, Chen Y, Kavehpour P, Segura T. Incorporation of active DNA/cationic polymer polyplexes into hydrogel scaffolds. Biomaterials 2010; 31(34) 9106-16.

[294] Yoo HS, Lee EA, Yoon JJ, Park TG. Hyaluronic acid modified biodegradable scaffolds for cartilage tissue engineering. Biomaterials 2005; 26(14) 1925-33.

[295] Flaibani M, Elvassore N. Gas anti-solvent precipitation assisted salt leaching for generation of micro- and nano-porous wall in bio-polymeric 3D scaffolds. Materials Science \& Engineering C, Materials for Biological Applications 2012; 32(6) 16329.

[296] Ko C-L, Tien Y-C, Wang J-C, Chen W-C. Characterization of controlled highly porous hyaluronan/gelatin cross-linking sponges for tissue engineering. Journal of the Mechanical Behavior of Biomedical Materials 2012; 14 227-38.

[297] Tokatlian T, Cam C, Siegman SN, Lei Y, Segura T. Design and characterization of microporous hyaluronic acid hydrogels for in vitro gene transfer to mMSCs. Acta Biomaterialia 2012; 8(11) 3921-31. 
[298] Cam C, Segura T. Chemical sintering generates uniform porous hyaluronic acid hydrogels. Acta Biomaterialia 2014; 10(1) 205-13.

[299] Mintz BR, Cooper JA. Hybrid hyaluronic acid hydrogel/poly( $\varepsilon$-caprolactone) scaffold provides mechanically favorable platform for cartilage tissue engineering studies. Journal of Biomedical Materials Research Part A 2013;

[300] Antunes JC, Oliveira JM, Reis RL, Soria JM, Gómez-Ribelles JL, Mano JF. Novel poly(L-lactic acid)/hyaluronic acid macroporous hybrid scaffolds: characterization and assessment of cytotoxicity. Journal of Biomedical Materials Research Part A 2010; 94(3) 856-69.

[301] Arnal-Pastor M, Vallés-Lluch A, Keicher M, Pradas MM. Coating typologies and constrained swelling of hyaluronic acid gels within scaffold pores. Journal of Colloid and Interface Science 2011; 361(1) 361-9.

[302] Liu Y, Ma G, Fang D, Xu J, Zhang H, Nie J. Effects of solution properties and electric field on the electrospinning of hyaluronic acid. Carbohydrate Polymers 2011; 83(2) 1011-5.

[303] Hsu F-Y, Hung Y-S, Liou H-M, Shen C-H. Electrospun hyaluronate-collagen nanofibrous matrix and the effects of varying the concentration of hyaluronate on the characteristics of foreskin fibroblast cells. Acta Biomaterialia 2010; 6(6) 21407.

[304] Arnal-Pastor M, Martínez Ramos C, Pérez Garnes M, Monleón Pradas M, Vallés Lluch A. Electrospun adherent-antiadherent bilayered membranes based on cross-linked hyaluronic acid for advanced tissue engineering applications. Materials Science \& Engineering C, Materials for Biological Applications 2013; 33(7) 4086-93.

[305] Ji Y, Ghosh K, Li B, Sokolov JC, Clark RAF, Rafailovich MH. Dual-syringe reactive electrospinning of cross-linked hyaluronic acid hydrogel nanofibers for tissue engineering applications. Macromolecular Bioscience 2006; 6(10) 811-7.

[306] Um IC, Fang D, Hsiao BS, Okamoto A, Chu B. Electro-spinning and electroblowing of hyaluronic acid. Biomacromolecules 2004; 5(4) 1428-36.

[307] Li J, He A, Han CC, Fang D, Hsiao BS, Chu B. Electrospinning of Hyaluronic Acid (HA) and HA/Gelatin Blends. Macromolecular Rapid Communications 2006; 27(2) 114-20.

[308] Fischer RL, McCoy MG, Grant SA. Electrospinning collagen and hyaluronic acid nanofiber meshes. Journal of Materials Science Materials in Medicine 2012; 23(7) 1645-54.

[309] Wang X, Um IC, Fang D, Okamoto A, Hsiao BS, Chu B. Formation of waterresistant hyaluronic acid nanofibers by blowing-assisted electro-spinning and nontoxic post treatments. Polymer 2005; 46(13) 4853-67.

[310] Sundararaghavan HG, Metter RB, Burdick JA. Electrospun fibrous scaffolds with multiscale and photopatterned porosity. Macromolecular Bioscience 2010; 10(3) 265-70.

[311] Kim TG, Chung HJ, Park TG. Macroporous and nanofibrous hyaluronic acid/collagen hybrid scaffold fabricated by concurrent electrospinning and deposition/leaching of salt particles. Acta Biomaterialia 2008; 4(6) 1611-9.

[312] Kim IL, Khetan S, Baker BM, Chen CS, Burdick JA. Fibrous hyaluronic acid hydrogels that direct MSC chondrogenesis through mechanical and adhesive cues. Biomaterials 2013; 34(22) 5571-80.

[313] Pescosolido L, Schuurman W, Malda J, Matricardi P, Alhaique F, Coviello T, et al. Hyaluronic acid and dextran-based semi-IPN hydrogels as biomaterials for bioprinting. Biomacromolecules 2011; 12(5) 1831-8.

[314] Suri S, Han L-H, Zhang W, Singh A, Chen S, Schmidt CE. Solid freeform fabrication of designer scaffolds of hyaluronic acid for nerve tissue engineering. Biomedical Microdevices 2011; 13(6) 983-93. 
[315] Park JK, Shim J-H, Kang KS, Yeom J, Jung HS, Kim JY, et al. Solid Free-Form Fabrication of Tissue-Engineering Scaffolds with a Poly(lactic-co-glycolic acid) Grafted Hyaluronic Acid Conjugate Encapsulating an Intact Bone Morphogenetic Protein-2/Poly(ethylene glycol) Complex. Advanced Functional Materials 2011; 21(15) 2906-12.

[316] Skardal A, Zhang J, McCoard L, Xu X, Oottamasathien S, Prestwich GD. Photocrosslinkable hyaluronan-gelatin hydrogels for two-step bioprinting. Tissue Engineering Part A 2010; 16(8) 2675-85.

[317] Takahashi S, Yamazoe H, Sassa F, Suzuki H, Fukuda J. Preparation of coculture system with three extracellular matrices using capillary force lithography and layer-by-layer deposition. Journal of Bioscience and Bioengineering 2009; 108(6) 544-50.

[318] Surendrakumar K, Martyn GP, Hodgers ECM, Jansen M, Blair JA. Sustained release of insulin from sodium hyaluronate based dry powder formulations after pulmonary delivery to beagle dogs. Journal of Controlled Release : Official Journal of the Controlled Release Society 2003; 91(3) 385-94.

[319] Yin D, Ge Z, Yang W, Liu C, Yuan Y. Inhibition of tumor metastasis in vivo by combination of paclitaxel and hyaluronic acid. Cancer Letters 2006; 243(1) 71-9.

[320] Coradini D, Zorzet S, Rossin R, Scarlata I, Pellizzaro C, Turrin C, et al. Inhibition of hepatocellular carcinomas in vitro and hepatic metastases in vivo in mice by the histone deacetylase inhibitor HA-But. Clinical Cancer Research: An Official Journal of the American Association for Cancer Research 2004; 10(14) 4822-30.

[321] Di Meo C, Panza L, Campo F, Capitani D, Mannina L, Banzato A, et al. Novel types of carborane-carrier hyaluronan derivatives via "click chemistry". Macromolecular Bioscience 2008; 8(7) 670-81.

[322] Varghese OP, Sun W, Hilborn J, Ossipov DA. In situ cross-linkable high molecular weight hyaluronan-bisphosphonate conjugate for localized delivery and cellspecific targeting: a hydrogel linked prodrug approach. Journal of the American Chemical Society 2009; 131(25) 8781-3.

[323] Kim KS, Hur W, Park S-J, Hong SW, Choi JE, Goh EJ, et al. Bioimaging for targeted delivery of hyaluronic Acid derivatives to the livers in cirrhotic mice using quantum dots. ACS Nano 2010; 4(6) 3005-14.

[324] Lee H, Choi SH, Park TG. Direct Visualization of Hyaluronic Acid Polymer Chain by Self-Assembled One-Dimensional Array of Gold Nanoparticles. Macromolecules 2006; 39(1) 23-5.

[325] Ito T, lida-Tanaka N, Niidome T, Kawano T, Kubo K, Yoshikawa K, et al. Hyaluronic acid and its derivative as a multi-functional gene expression enhancer: protection from non-specific interactions, adhesion to targeted cells, and transcriptional activation. Journal of Controlled Release : Official Journal of the Controlled Release Society 2006; 112(3) 382-8.

[326] Jiang G, Park K, Kim J, Kim KS, Hahn SK. Target specific intracellular delivery of siRNA/PEI-HA complex by receptor mediated endocytosis. Molecular Pharmaceutics 2009; 6(3) 727-37.

[327] Kim SJ, Hahn SK, Kim MJ, Kim DH, Lee YP. Development of a novel sustained release formulation of recombinant human growth hormone using sodium hyaluronate microparticles. Journal of Controlled Release : Official Journal of the Controlled Release Society 2005; 104(2) 323-35.

[328] Motokawa K, Hahn SK, Nakamura T, Miyamoto H, Shimoboji T. Selectively crosslinked hyaluronic acid hydrogels for sustained release formulation of erythropoietin. Journal of Biomedical Materials Research Part A 2006; 78(3) 45965.

[329] De la Fuente M, Seijo B, Alonso MJ. Design of novel polysaccharidic nanostructures for gene delivery. Nanotechnology 2008; 19(7) 075105. 
[330] Lee H, Ahn C-H, Park TG. Poly[lactic-co-(glycolic acid)]-grafted hyaluronic acid copolymer micelle nanoparticles for target-specific delivery of doxorubicin. Macromolecular Bioscience 2009; 9(4) 336-42.

[331] Pitarresi G, Palumbo FS, Albanese A, Fiorica C, Picone P, Giammona G. Selfassembled amphiphilic hyaluronic acid graft copolymers for targeted release of antitumoral drug. Journal of Drug Targeting 2010; 18(4) 264-76.

[332] Li J, Huo M, Wang J, Zhou J, Mohammad JM, Zhang Y, et al. Redox-sensitive micelles self-assembled from amphiphilic hyaluronic acid-deoxycholic acid conjugates for targeted intracellular delivery of paclitaxel. Biomaterials 2012; 33(7) 2310-20.

[333] Almalik A, Donno R, Cadman CJ, Cellesi F, Day PJ, Tirelli N. Hyaluronic acidcoated chitosan nanoparticles: molecular weight-dependent effects on morphology and hyaluronic acid presentation. Journal of Controlled Release : Official Journal of the Controlled Release Society 2013; 172(3) 1142-50.

[334] Ratliff BB, Ghaly T, Brudnicki P, Yasuda K, Rajdev M, Bank M, et al. Endothelial progenitors encapsulated in bioartificial niches are insulated from systemic cytotoxicity and are angiogenesis competent. American Journal of Physiology Renal Physiology 2010; 299(1) F178-86.

[335] Zor F, Deveci M, Kilic A, Ozdag MF, Kurt B, Sengezer M, et al. Effect of VEGF gene therapy and hyaluronic acid film sheath on peripheral nerve regeneration. Microsurgery 2014; 34(3) 209-16.

[336] Shibata S, Cho KH, Kim JH, Abe H, Murakami G, Cho BH. Expression of hyaluronan (hyaluronic acid) in the developing laminar architecture of the human fetal brain. Annals of Anatomy = Anatomischer Anzeiger : Official Organ of the Anatomische Gesellschaft 2013; 195(5) 424-30.

[337] Preston M, Sherman LS. Neural stem cell niches: roles for the hyaluronan-based extracellular matrix. Frontiers in Bioscience (Scholar Edition) 2011; 3 1165-79.

[338] Wang Y, Wei YT, Zu ZH, Ju RK, Guo MY, Wang XM, et al. Combination of hyaluronic acid hydrogel scaffold and PLGA microspheres for supporting survival of neural stem cells. Pharmaceutical Research 2011; 28(6) 1406-14.

[339] Wei Y-T, He Y, Xu C-L, Wang Y, Liu B-F, Wang X-M, et al. Hyaluronic acid hydrogel modified with nogo-66 receptor antibody and poly-L-lysine to promote axon regrowth after spinal cord injury. Journal of Biomedical Materials Research Part B, Applied Biomaterials 2010; 95(1) 110-7.

[340] Shoichet MS, Tator CH, Poon P, Kang C, Baumann MD. Intrathecal drug delivery strategy is safe and efficacious for localized delivery to the spinal cord. Progress in Brain Research 2007; 161 385-92.

[341] Kang CE, Poon PC, Tator CH, Shoichet MS. A new paradigm for local and sustained release of therapeutic molecules to the injured spinal cord for neuroprotection and tissue repair. Tissue Engineering Part A 2009; 15(3) 595604.

[342] Yu H, Fang Y, Chen L, Chen S. Investigation of redox initiators for free radical frontal polymerization. Polymer International 2009; 58(8) 851-7.

[343] Brígido Diego R, Salmerón Sánchez M, Gómez Ribelles JL, Monleón Pradas M. Effect of gamma-irradiation on the structure of poly(ethyl acrylate-co-hydroxyethyl methacrylate) copolymer networks for biomedical applications. Journal of Materials Science Materials in Medicine 2007; 18(5) 693-8.

[344] Martínez-Ramos C, Rodríguez-Pérez E, Garnes MP, Chachques JC, Moratal D, Vallés-Lluch A, et al. Design and Assembly Procedures for Large-Sized Biohybrid Scaffolds as Patches for Myocardial Infarct. Tissue Engineering Part C, Methods 2014; 20(10) 817-27.

[345] Mark J. Polymer data handbook. Oxford (UK): Oxford University Press, Inc.; 1999. 
[346] Ohara T, Sato T, Shimizu N, Prescher G, Schwind H, Weiberg O, et al. Ullmann's Encyclopedia of Industrial Chemistry. Weinheim (Germany): Wiley-VCH Verlag $\mathrm{GmbH} \&$ Co. KGaA; 2000.

[347] Lozano Picazo P, Pérez Garnes M, Martínez Ramos C, Vallés-Lluch A, Monleón Pradas M. New Semi-Biodegradable Materials from Semi-Interpenetrated Networks of Poly( $\epsilon$-caprolactone) and Poly(ethyl acrylate). Macromolecular Bioscience; Doi: 101002/mabi201400331 n.d.;

[348] Khan AJ, Percival SP. 12 year results of a prospective trial comparing poly(methyl methacrylate) and poly(hydroxyethyl methacrylate) intraocular lenses. Journal of Cataract and Refractive Surgery 1999; 25(10) 1404-7.

[349] Skalsky B, Petereit H-U. Chemistry and Application Properties of Polymethacrylate Systems. In: Felton JWM, Linda A, editors. Aqueous Polymeric Coatings for Pharmaceutical Dosage Forms, CRC Press; 2008.

[350] Patil SB, Naveen BH, Patil NP. Bonding acrylic teeth to acrylic resin denture bases: a review. Gerodontology 2006; 23(3) 131-9.

[351] Song M, Uhrich KE. Optimal micropattern dimensions enhance neurite outgrowth rates, lengths, and orientations. Annals of Biomedical Engineering 2007; 35(10) 1812-20.

[352] Moore K, MacSween M, Shoichet M. Immobilized concentration gradients of neurotrophic factors guide neurite outgrowth of primary neurons in macroporous scaffolds. Tissue Engineering 2006; 12(2) 267-78.

[353] Soria JM, Martínez Ramos C, Bahamonde O, García Cruz DM, Salmerón Sánchez M, García Esparza MA, et al. Influence of the substrate's hydrophilicity on the in vitro Schwann cells viability. Journal of Biomedical Materials Research Part A 2007; 83(2) 463-70.

[354] Campillo-Fernández AJ, Unger RE, Peters K, Halstenberg S, Santos M, Salmerón Sánchez M, et al. Analysis of the biological response of endothelial and fibroblast cells cultured on synthetic scaffolds with various hydrophilic/hydrophobic ratios: influence of fibronectin adsorption and conformation. Tissue Engineering Part $A$ 2009; 15(6) 1331-41.

[355] Lesný P, De Croos J, Prádný M, Vacík J, Michálek J, Woerly S, et al. Polymer hydrogels usable for nervous tissue repair. Journal of Chemical Neuroanatomy 2002; 23(4) 243-7.

[356] Cui FZ, Tian WM, Fan YW, Hou SP, Xu QY, Lee I-S. Cerebrum Repair with PHPMA Hydrogel Immobilized with Neurite-Promoting Peptides in Traumatic Brain Injury of Adult Rat Model. Journal of Bioactive and Compatible Polymers 2003; 18(6) 413-32.

[357] Woerly S, Pinet E, de Robertis L, Van Diep D, Bousmina M. Spinal cord repair with PHPMA hydrogel containing RGD peptides (NeuroGel). Biomaterials 2001; 22(10) 1095-111.

[358] Loh NK, Woerly S, Bunt SM, Wilton SD, Harvey AR. The regrowth of axons within tissue defects in the CNS is promoted by implanted hydrogel matrices that contain BDNF and CNTF producing fibroblasts. Experimental Neurology 2001; 170(1) 72 84.

[359] Hejcl A, Lesný P, Prádný M, Michálek J, Jendelová P, Stulík J, et al. Biocompatible hydrogels in spinal cord injury repair. Physiological Research / Academia Scientiarum Bohemoslovaca 2008; 57(Suppl 3) S121-32.

[360] Ward IM, Hadley DW. Introduction to the Mechanical Properties of Solid Polymers. London: Wiley; 1993.

[361] Ganji F, Vasheghani-Farahani S, Vasheghani-Raraahani E. Theoretical description of hydrogel swelling: a review. Iranian Polymer Journal 2010; 19(5) 375-98.

[362] Flory PJ. Phase equilibria in polymer systems. Principles of Polymer Chemistry, Ithaca, (NY): Cornell University Press; 1953, p. 541-94. 
[363] Grassi M, Grassi G. Mathematical modelling and controlled drug delivery: matrix systems. Current Drug Delivery 2005; 2(1) 97-116.

[364] Bercea M, Nichifor M, Eckelt J, Wolf BA. Dextran-Based Polycations: Thermodynamic Interaction with Water as Compared With Unsubstituted Dextran, 2 - Flory/Huggins Interaction Parameter. Macromolecular Chemistry and Physics $2011 ; \mathrm{n} / \mathrm{a}-\mathrm{n} / \mathrm{a}$.

[365] Gigante A, Callegari L. The role of intra-articular hyaluronan (Sinovial) in the treatment of osteoarthritis. Rheumatology International 2011; 31(4) 427-44.

[366] Patenaude M, Hoare T. Injectable, mixed natural-synthetic polymer hydrogels with modular properties. Biomacromolecules 2012; 13(2) 369-78.

[367] Collins MN, Birkinshaw C. Investigation of the swelling behavior of crosslinked hyaluronic acid films and hydrogels produced using homogeneous reactions. Journal of Applied Polymer Science 2008; 109(2) 923-31.

[368] Mrácek A, Benesová K, Minarík A, Urban P, Lapcík L. The diffusion process of sodium hyaluronate $(\mathrm{Na}-\mathrm{Ha})$ and $\mathrm{Na}-\mathrm{Ha}-\mathrm{n}$-alkyl derivatives films swelling. Journal of Biomedical Materials Research Part A 2007; 83(1) 184-90.

[369] Credi C, Biella S, De Marco C, Levi M, Suriano R, Turri S. Fine tuning and measurement of mechanical properties of crosslinked hyaluronic acid hydrogels as biomimetic scaffold coating in regenerative medicine. Journal of the Mechanical Behavior of Biomedical Materials 2014; 29 309-16.

[370] Kim SJ, Lee CK, Kim SI. Characterization of the water state of hyaluronic acid and poly(vinyl alcohol) interpenetrating polymer networks. Journal of Applied Polymer Science 2004; 92(3) 1467-72.

[371] O'Brien FJ, Harley BA, Yannas I V, Gibson LJ. The effect of pore size on cell adhesion in collagen-GAG scaffolds. Biomaterials 2005; 26(4) 433-41.

[372] Lai J. Relationship between structure and cytocompatibility of divinyl sulfone cross-linked hyaluronic acid. Carbohydrate Polymers 2014; 30(101) 203-12.

[373] Oudshoorn MHM, Rissmann R, Bouwstra JA, Hennink WE. Synthesis of methacrylated hyaluronic acid with tailored degree of substitution. Polymer 2007; 48(7) 1915-20.

[374] Zhang C, Ren Z, Liu L, Yin Z. Modelling hydrogen bonds in N N dimethylformamide. Molecular Simulation 2013; 39(11) 875-81.

[375] Güner A. The algorithmic calculations of solubility parameter for the determination of interactions in dextran/certain polar solvent systems. European Polymer Journal 2004; 40(7) 1587-94.

[376] Gianotti V, Antonioli D, Sparnacci K, Laus M, Giammaria TJ, Ferrarese Lupi F, et al. On the Thermal Stability of PS- b -PMMA Block and P(S- $r-M M A)$ Random Copolymers for Nanopatterning Applications. Macromolecules 2013; 46(20) 8224-34.

[377] Campillo-Fernandez AJ, Pastor S, Abad-Collado M, Bataille L, Gomez-Ribelles $\mathrm{JL}$, Meseguer-Dueñas JM, et al. Future design of a new keratoprosthesis. Physical and biological analysis of polymeric substrates for epithelial cell growth. Biomacromolecules 2007; 8(8) 2429-36.

[378] Dickie R. Mechanical properties (small deformations) of multiphase polymer blends. In: Paul D, Newman S, editors. Polymer Blends, San Diego, CA: Academic Press, Inc; 1978, p. 353-88.

[379] Xiao L, Tong Z, Chen Y, Pochan DJ, Sabanayagam CR, Jia X. Hyaluronic acidbased hydrogels containing covalently integrated drug depots: implication for controlling inflammation in mechanically stressed tissues. Biomacromolecules 2013; 14(11) 3808-19.

[380] Georges PC, Miller WJ, Meaney DF, Sawyer ES, Janmey PA. Matrices with compliance comparable to that of brain tissue select neuronal over glial growth in mixed cortical cultures. Biophysical Journal 2006; 90(8) 3012-8. 
[381] Hernández-Hernández OT, González-García TK, Camacho-Arroyo I. Progesterone receptor and SRC-1 participate in the regulation of VEGF, EGFR and Cyclin D1 expression in human astrocytoma cell lines. The Journal of Steroid Biochemistry and Molecular Biology 2012; 132(1-2) 127-34.

[382] Watcharasit $P$, Visitnonthachai D, Suntararuks S, Thiantanawat A, Satayavivad J. Low arsenite concentrations induce cell proliferation via activation of VEGF signaling in human neuroblastoma SH-SY5Y cells. Environmental Toxicology and Pharmacology 2012; 33(1) 53-9.

[383] Rico P, Rodríguez Hernández JC, Moratal D, Altankov G, Monleón Pradas M, Salmerón-Sánchez M. Substrate-induced assembly of fibronectin into networks: influence of surface chemistry and effect on osteoblast adhesion. Tissue Engineering Part A 2009; 15(11) 3271-81. 



\section{Abbreviations}

\begin{tabular}{|c|c|}
\hline${ }^{1} \mathrm{H}-\mathrm{NMR}$ & Proton Nuclear Magnetic Resonance \\
\hline$A C$ & Alternating Current \\
\hline $\mathrm{ADH}$ & Adipic Dihydrazide \\
\hline ALS & Amyotrophic Lateral Sclerosis \\
\hline ASA & Aryl/Alkyl Succinic Anhydride \\
\hline ATCC & American Type Culture Collection \\
\hline ATR-FTIR & $\begin{array}{l}\text { Attenuated Total Reflectance Fourier Transformed Infrared } \\
\text { spectroscopy }\end{array}$ \\
\hline BBB & Blood Brain Barrier \\
\hline BDDE & Butanediol-Diglycidyl Ether \\
\hline BDNF & Brain-Derived Neurotrophic Factor \\
\hline bFGF & basic Fibroblast Growth Factor \\
\hline BMP & Bone Morphogenetic Protein \\
\hline BSCB & Blood Spinal Cord Barrier \\
\hline CD-31 & Cluster of Differentiation 31 \\
\hline CD44 & Cell-Surface Glycoprotein 44 \\
\hline CDI & 1,1'-Carbonyldiimidazole \\
\hline CDMT & 2-Chloro-Dimethoxy-1,3,5-Triazine \\
\hline CMPI & 2-Chloro-1-Methylpyridinium lodide \\
\hline CNF & Carbon Nanofibre \\
\hline CNS & Central Nervous System \\
\hline CNT & Carbon Nanotube \\
\hline CSPG & Chondroitin Sulphate Proteoglycan \\
\hline CTA & Cetyltrimethylammonium Bromide \\
\hline DC & Direct Current \\
\hline DMEM/F12 & Dulbecco's Modified Eagle Medium/nutrient mixture F-12 \\
\hline DMF & Dimethylformamide \\
\hline DMSO & Dimethyl Sulfoxide \\
\hline DMTA & Dynamic Mechanical Thermal Analysis \\
\hline DRG & Dorsal Root Ganglia \\
\hline
\end{tabular}


Dithiothreitol

DVS

Divinyl Sulfone

EA

Ethyl Acrylate

ECBM

Endothelial Cell Basal Medium

ECM

Extracellular Matrix

EDC

1-Ethyl-3-(3-Dimethylaminopropyl)Carbodiimide

EGF

Endothelial Growth Factor

EPO

Erythropoietin

ESC

Embryonic Stem Cell

FCS

Foetal Calf Serum

FDA

Food and Drug Administration

FGF

Fibroblast Growth Factor

FN

Fibronectin

FPRL1

Formyl Peptide Receptor Like 1

FVM

Foetal Ventral Mesencephalon

GAG

Glycosaminoglycan

GDNF

Glial-Derived Neurotrophic Factor

GFAP

Glial Fibrillary Acidic Protein

GLcA

D-Glucuronic Acid

GLCNAC

N-Acetyl-D-Glucosamine

GM

Glycidyl Methacrylate

GPC

Gel Permeation Chromatography

HA

Hyaluronan

HA:DVS

Molar ratio of hyaluronan monomeric unit to divinyl sulfone

HA-DTPH

3,3'-Dithiobis(propanoic dihydrazide)-modified HA

HA-DVS

Hyaluronan crosslinked with divinyl sulfone

HAGM

HA with Glycidyl Methacrylate groups

HAMC

HA with Methylcellulose

HARE

Hyaluronan Receptor for Endocytosis

hCMEC/D3

human Cerebral Microvascular Endothelial Cell line 


\begin{tabular}{|c|c|}
\hline HD & Huntington's Disease \\
\hline HEPES & Hydroxyethyl Piperazine Ethanesulfonic acid \\
\hline hGH & human Growth Hormone \\
\hline $\mathrm{HOBt}$ & 1-Hydroxybenzotriazole \\
\hline HRP & Horse-Radish Peroxidase \\
\hline hRPE & human Retinal Pigment Epithelium Cell \\
\hline HYAL & Hyaluronidases \\
\hline Hyal & Hyaluronoglucosaminidase \\
\hline IPN & Interpenetrating Polymer Network \\
\hline iPS & induced Pluripotent Stem Cell \\
\hline LALS & Low Angle Light Scattering \\
\hline LDH & Lactate Dehydrogenase \\
\hline LEC receptor & Liver Endothelial Cell Clearance Receptor \\
\hline LN & Laminin \\
\hline LPS & Lipopolysaccharide \\
\hline lyo_HA:DVS & Lyophylized hyaluronan crosslinked with divinyl sulfone \\
\hline LYVE1 & Lymphatic Vessel Endothelial HA Receptor \\
\hline MA & Methacrylic Anhydride \\
\hline MAG & Myelin Associated Glycoprotein \\
\hline MALS & Multi-Angle Light Scattering \\
\hline MBAA & N,N'-Methylenebisacrylamide \\
\hline MeHA & Methacrylated Hyaluronan \\
\hline MeHA:PEA & $\begin{array}{l}\text { Weight ratio of methacrylated hyaluronan to ethyl acrylate in } \\
\text { the copolymerization }\end{array}$ \\
\hline MEM & Minimum Essential Media \\
\hline MSC & Mesenchymal Stem Cell \\
\hline NHS & N-Hydroxysuccinimide \\
\hline $\mathrm{NIH}$ & National Institutes of Health \\
\hline NMM & N-Methylmorpholinium \\
\hline NPC & Neural Progenitor Cell \\
\hline NSC & Neural Stem Cells \\
\hline
\end{tabular}


NT-3

OECs

PANI

pBEC

PBS

PBSA

PCL

PCLF

PD

PDL

PDMS

PDS

pDVS

PEA

PEDOT

PEG

PEMA

PGA

PHEMA

PHPMA

PLGA

PLLA

PMA

PMMA

PNS

poly(MeHA-co-EA)

PPy

RHAMM

ROS

SAPN

SC
Neurotrophin-3

Olfactory Ensheathing Cell

Polyaniline

pig Brain Endothelial Cell

Phosphate Buffered Saline.

$1 \%$ foetal calf serum in $0.1 \mathrm{M}$ PBS

Poly- $\varepsilon$-caprolactone

Poly( $\varepsilon$-caprolactone fumarate)

Parkinson's Disease

Poly-D-Lysine

Poly(dimethylsiloxane)

Polydioxanone

Poly(divinyl sulfone)

Poly(ethyl acrylate)

Poly(3,4-ethylenedioxythiphene)

Poly(ethylene glycol)

Poly(ethyl methacrylate)

Polyglycolic acid

Poly(hydroxyethyl methacrylate)

Poly(hydroxypropyl methacrylate)

Poly(lactic-co-glycolic acid)

Poly(L-lactic acid)

Poly(methyl acrylate)

Poly(methyl methacrylate)

Peripheral Nervous System

Poly(methacrylated hyaluronan-co-ethyl acrylate)

Polypirrol

Receptor for Hyaluronate-Mediated Motility

Reactive Oxygen Species

Self-Assembling Peptides Nanofibre

Schwann Cell 
Scaff_HA:DVS

$\mathrm{SCl}$

SDR-FTIR

SEC

SEM

SH-SY5Y

Solv_HA:DVS

SVZ

TACC

TBA

TBI

TCD

TGA

TLR-4/2

Tuj-1

U373

VEGF

WI_HA:DVS
Scaffolds of hyaluronan crosslinked with divinyl sulfone Spinal Cord Injury

Smart Diffuse Reflectance Fourier Transform Infrared spectroscopy

Size Exclusion Chromatography

Scanning Electron Microscope

Neuroblastoma Cell Line

Solvent-cast polymer networks of hyaluronan crosslinked with divinyl sulfone

Subventricular Zone

Transforming Acidic Coiled-Coil domain

Tetrabutylammonium

Traumatic Brain Injury

Thermal Conductivity Detector

Thermogravimetric Analysis

Toll-Like Receptors

Neuron-specific class III beta-tubulin

Human glioblastoma-astrocytoma cell line

Vein Endothelial Growth Factor

Hyaluronan crosslinked with divinyl sulfone subjected to a phase inversion of water 


\section{Glossary of symbols}

$\overline{\mathrm{M}}_{\mathrm{c}}$

$\bar{d}$

$\mathrm{e}_{0}$

$\mathrm{V}_{\mathrm{H}_{2} \mathrm{O}}$

$\overline{\mathrm{v}}$

$\Phi_{2}$ and $\Phi_{0}$

A

$\mathrm{Cs}^{*}$

D

$d_{d}$

$d_{s}$

$\mathrm{E}$ or $\mathrm{E}_{0}$

E'

E”

$E_{f}$

EWC

$\mathrm{F}$

i

K

I

Mo

$\mathrm{m}_{\text {HA }}$

$\mathrm{Mi}_{\mathrm{i}}$

$\mathrm{Mn}$

moctane

$\mathrm{M}_{\mathrm{t}}$

$\mathrm{Mw}$

$\mathrm{n}$

PDI
Molecular weight between crosslinking points

Disaccharide units between crosslinking points

Initial thickness of the sample (compression tests)

Specific volume of water

Specific volume of hyaluronan

Polymer fractions in the hydrogel at equilibrium swelling and after the crosslinking reaction

Initial cross-section of the sample (compression tests)

Salt concentration outside the hydrogel.

Diffusion coefficient

Diameter of the dry samples

Diameter of the swollen samples at equilibrium

Initial modulus (determined at strains between 0 and 0.1 )

Storage modulus

Loss modulus

Final modulus (determined between 3.5 and $4 \mathrm{kPa}$ )

Equilibrium Water Content,

Force (compression tests)

Degree of ionization of hyaluronan

Swelling constant

Thickness of the hydrogel

Mass of the xerogel

Mass of dry hyaluronan

Molecular weight

Number average Molecular weight

Mass of octane (porosity experiments)

Amount of water in the swollen hydrogel at time $t$

Weight average Molecular weight

Swelling exponent

Polydispersity Index 


\begin{tabular}{|c|c|}
\hline Q & Reciprocal of hyaluronan volume fraction \\
\hline Qisotropic & $\begin{array}{l}\text { Reciprocal of hyaluronan volume fraction determined from the } \\
\text { measure of diameter }\end{array}$ \\
\hline$Q_{M}$ & Degree of mass swelling at equilibrium \\
\hline SW & Weight ratio of water to xerogel \\
\hline $\tan \delta$ & Loss factor \\
\hline $\operatorname{Tg}$ & Temperature of glass transition \\
\hline $\mathrm{V}_{1}$ & Molar volume of water \\
\hline$V_{d}$ & Volume of the dry polymer network \\
\hline $\mathrm{V}_{\mathrm{HA}}$ & Volume of hyaluronan in the sample (porosity experiments) \\
\hline$V_{\text {macropores }}$ & Volume of the large pores (porosity experiments) \\
\hline Voctane & Specific volume of octane \\
\hline$V_{\text {pores }}$ & Volume of the small pores (porosity experiments) \\
\hline $\mathrm{V}_{\mathrm{s}}$ & Volume of the swollen polymer network at equilibrium \\
\hline $\mathrm{V}_{\mathrm{t}}$ & Total volume of the samples (porosity experiments) \\
\hline $\mathrm{V}_{\mathrm{u}}$ & Molar volume of ionisable groups per HA disaccharide unit \\
\hline $\mathrm{wa}_{\mathrm{a}}$ & Mass of the sample in air (density experiments) \\
\hline$w_{d}$ & Mass of xerogel \\
\hline$W_{E A}$ & $\begin{array}{l}\text { Nominal poly(ethyl acrylate) weight fraction in the copolymer } \\
\text { networks }\end{array}$ \\
\hline $\mathrm{w}_{\mathrm{i}}$ & Fraction of each specie \\
\hline Woctane & Mass of each sample in octane (density experiments) \\
\hline WPEA & $\begin{array}{l}\text { Experimental poly(ethyl acrylate) weight fraction in the } \\
\text { copolymer networks }\end{array}$ \\
\hline Ws & Mass of hydrogel \\
\hline$\Delta \mathrm{e}$ & Change in thicknes (compression tests) \\
\hline$\varepsilon$ & Strain (compression tests) \\
\hline$\varepsilon_{f}$ & Interpolation of the final modulus slope with the strain axis \\
\hline$\lambda x, \lambda y, \lambda z$ and $\Lambda$ & Stretching ratios between the swollen and dry samples \\
\hline$\pi \mathrm{m}$ & Macroporosity \\
\hline$\pi \mathrm{p}$ & Porosity \\
\hline$\rho$ & Density \\
\hline
\end{tabular}




$\begin{array}{ll}\rho_{\mathrm{HA}} & \text { Density of hyaluronan } \\ \rho_{\text {octane }} & \text { Density of octane at } 25^{\circ} \mathrm{C} \\ \rho_{\mathrm{x}} & \text { Crosslinking density } \\ \sigma & \text { Tension (compression tests) } \\ \Phi & \text { Hyaluronan volume fraction } \\ X & \text { Flory polymer-solvent interaction parameter }\end{array}$


